\title{
Direct Synthesis of Alkenyl Boronic Esters from Unfunctionalized Alkenes: A Boryl-Heck Reaction
}

\author{
William B. Reid, Jesse J. Spillane, Sarah B. Krause and Donald A. Watson* \\ Department of Chemistry and Biochemistry, University of Delaware, \\ Newark, Delaware 19716, United States
}

\section{Supporting Information}

Index

1. General Experimental Details: $\quad$ S2

2. Instrumentation and Chromatography: $\quad$ S2

3. Synthesis of Non-commercial Starting Materials and Reagents: S3

4. General Procedure for the a-Olefin Boryl-Heck Reaction: S5

5. General Procedure for the Styrene Boryl-Heck Reaction: S11

6. Additional Optimization Data: $\quad \mathrm{S16}$

7. Computational and Experimental Mechanistic Insight: S18

8. Crystallographic Details: $\quad$ S21

9. References: $\quad$ S22

10. Spectral Data: $\quad$ S23 


\section{General Experimental Details:}

Diethyl ether, tetrahydrofuran and dichloromethane were dried on alumina according to published procedures. ${ }^{1}$ Catechol was purchased from Alfa Aesar and recrystallized from hot toluene prior to use. Boron trichloride was purchased from Acros as a $1 \mathrm{M}$ solution in hexanes and used as received. N,N-Dicyclohexylmethylamine was purchased from $\mathrm{TCl}$, distilled from calcium hydride $\left(80^{\circ} \mathrm{C}, 150 \mathrm{mtorr}\right)$ and stored at $\mathrm{rt}$ on the bench in a nitrogen-filled Strauss flask. Trifluorotoluene was purchased from Sigma Aldrich in an anhydrous septum sealed bottle, transferred to a Straus flask by cannula transfer and sparged with nitrogen for 15 minutes. Lithium trifluoromethanesulfonate was purchased from Oakwood and stored in a nitrogen-filled glovebox or in a desiccator under air. Bis(trimethylsilylmethyl)-(cycloocta-1,5-diene) palladium(II) $\left[(\mathrm{COD}) \mathrm{Pd}\left(\mathrm{CH}_{2} \mathrm{SiMe}_{3}\right)_{2}\right]$ was prepared according to a published procedure. ${ }^{2}$ Tris(dibenzylideneacetone)dipalladium $(0)\left[\mathrm{Pd}_{2}(\mathrm{dba})_{3}\right]$ was purchased from Aldrich or Strem and used as received. Bis(3,5-di-tert-butylphenyl)(tert-butyl)phosphine (L1) was prepared according to a published procedure. ${ }^{3}$ Substrates ethyl 4-vinylbenzoate, ${ }^{4}$ 5-vinylbenzo-1,3-dioxole ${ }^{5}$ and 1 (toluene-4-sulfonyl)-2-vinyl-1H-indole ${ }^{6}$ were prepared according to published literature procedures. All other substrates and reagents were purchased in highest analytical purity from commercial suppliers and used as received. Vials used in the glovebox were dried in a gravity oven at $140{ }^{\circ} \mathrm{C}$ for a minimum of $12 \mathrm{~h}$, transferred into the glovebox hot, and then stored at $\mathrm{rt}$ in the glovebox prior to use. All other glassware was flame-dried under vacuum prior to use. "Double manifold" refers to a standard Schlenk-line gas manifold equipped with nitrogen and vacuum (ca. 100 mtorr). All optimization reactions $(0.25 \mathrm{mmol})$ were run in a nitrogen-filled glovebox and heated using an aluminum block on a magnetic stir plate. All yields in optimization reactions were determined using ${ }^{1} \mathrm{H}$ NMR using 1,3,5-trimethoxybenzene or ferrocene as an internal standard and $E / Z$ ratios were determined using ${ }^{1} \mathrm{H}$ NMR of unpurified products. All other reactions were set up using standard Schlenk technique and heated with stirring in temperature controlled oil baths. Note: Any product yields listed in the main text that do not match those listed in the supporting information are the average of multiple isolated yields. The $E / Z$ ratio of the isolated products may differ from the crude mixture due to enrichment during purification. Only the $E$ isomer is reported for the ${ }^{1} \mathrm{H}$ and ${ }^{13} \mathrm{C}$ NMR spectral data. The ${ }^{13} \mathrm{C}$ NMR spectra may contain extra, unassigned peaks, which we attribute to the minor $Z$ isomer. Note: The ${ }^{13} \mathrm{C}$ NMR signal for carbons attached to boron did not appear in the collected spectra due to the quadruple splitting of ${ }^{11} B{ }^{7}$ NMR data for some compounds may be reported in two different solvents to resolve overlapped ${ }^{13} \mathrm{C}$ peaks.

\section{Instrumentation and Chromatography:}

$400 \mathrm{MHz}{ }^{1} \mathrm{H}, 101 \mathrm{MHz}{ }^{13} \mathrm{C}$ and $376 \mathrm{MHz}{ }^{19} \mathrm{~F}$ spectra were obtained on a $400 \mathrm{MHz}$ FT-NMR spectrometer equipped with a Bruker CryoPlatform. $600 \mathrm{MHz}{ }^{1} \mathrm{H}, 151 \mathrm{MHz}{ }^{13} \mathrm{C}$, and $193 \mathrm{MHz}{ }^{11} \mathrm{~B}$ spectra were obtained on a $600 \mathrm{MHz}$ FT-NMR spectrometer equipped with a Bruker SMART probe. All samples were analyzed in the indicated deutero-solvent and were recorded at ambient temperatures. All chemical shifts are reported in ppm. ${ }^{1} \mathrm{H}$ NMR spectra were calibrated using the residual protio-signal in deutero-solvents as a standard. ${ }^{13} \mathrm{C}$ NMR spectra were calibrated using the deutero-solvent as a standard. IR spectra were recorded on a Nicolet Magma-IR 560 FT-IR spectrometer as thin films on $\mathrm{KBr}$ plates. High resolution MS data was obtained on a Thermo QExactive Orbitrap using electrospray ionization (ESI), or a Waters GCT Premier spectrometer using chemical ionization $(\mathrm{Cl})$ or liquid injection field desorption ionization (LIFDI). Column chromatography was performed with boric acid impregnated 40-63 $\mu \mathrm{m}$ silica gel (see below) with the eluent reported in parentheses. Analytical thin-layer chromatography (TLC) was performed on pre-coated glass plates and visualized by UV or by staining with iodine or $\mathrm{KMnO}_{4}$. 


\section{Synthesis of Non-commercial Starting Materials and Reagents:}

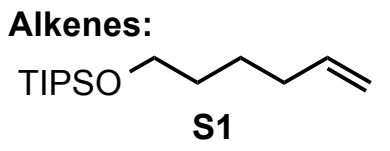

(S1) A $500 \mathrm{~mL}$ round bottom flask equipped with a magnetic stir bar and rubber septum was charged with 4-dimethylaminopyridine (305 $\mathrm{mg}, 0.1$ equiv), triethylamine $(100 \mathrm{~mL})$, dichloromethane $(100 \mathrm{~mL})$, and 5-hexenol ( $3.0 \mathrm{~mL}, 25 \mathrm{mmol}, 1.0$ equiv) and sealed under air. Triisopropylchlorosilane $(6.7 \mathrm{~mL}$, $31.3 \mathrm{mmol}, 1.25$ equiv) was then added dropwise via syringe and the reaction was stirred for 72 h. The reaction was opened to air, quenched with water $(100 \mathrm{~mL})$, and extracted with diethyl ether $(3 \times 100 \mathrm{~mL})$. The combined organic layers were washed with $1 \mathrm{M}$ hydrochloric acid (100 $\mathrm{mL}$ ), dried with $\mathrm{MgSO}_{4}$, filtered through Celite, and concentrated in vacuo. The crude oil was purified by flash silica gel chromatography (hexanes) to afforded $\mathbf{S 1}$ as a colorless oil $(5.32 \mathrm{~g}$, 83\%): ${ }^{1} \mathrm{H}$ NMR $\left(400 \mathrm{MHz}, \mathrm{CDCl}_{3}\right) \delta 5.82(\mathrm{ddt}, J=16.9,10.2,6.6 \mathrm{~Hz}, 1 \mathrm{H}), 5.01$ (dd, $J=17.1,1.7$ $\mathrm{Hz}, 1 \mathrm{H}), 4.94(\mathrm{~d}, J=10.2 \mathrm{~Hz}, 1 \mathrm{H}), 3.69(\mathrm{t}, J=6.4 \mathrm{~Hz}, 2 \mathrm{H}), 2.08(\mathrm{q}, J=7.1 \mathrm{~Hz}, 2 \mathrm{H}), 1.62-1.52$ $(\mathrm{m}, 2 \mathrm{H}), 1.51-1.41(\mathrm{~m}, 2 \mathrm{H}), 1.14-0.96(\mathrm{~m}, 21 \mathrm{H}) ;{ }^{13} \mathrm{C}$ NMR $\left(101 \mathrm{MHz}, \mathrm{CDCl}_{3}\right) \delta 139.1,114.5$, 63.4, 33.8, 32.6, 25.3, 18.2, 12.2; FTIR $\left(\mathrm{cm}^{-1}\right): 2942$, 2892, 2866, 1463, 1106, 1070, 1013, 994, $910,882,680,658$. HRMS $(\mathrm{Cl}) \mathrm{m} / \mathrm{z}$, calcd for $\left[\mathrm{C}_{15} \mathrm{H}_{33} \mathrm{OSi}\right]^{+}: 257.2301$; found: $257.2296{ }^{8}$

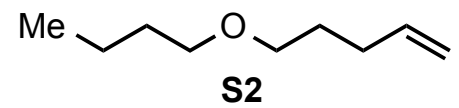

(S2) A $500 \mathrm{~mL}$ round bottom flask equipped with a magnetic stir bar and a rubber septum was flame dried under vacuum, cooled to $\mathrm{rt}$ and refilled with nitrogen. The flask was briefly opened to air, and quickly charged with sodium hydride $60 \%$ dispersion in mineral oil $(3.5 \mathrm{~g}, 87.5 \mathrm{mmol}, 1.5$ equiv), and the flask was resealed. Anhydrous tetrahydrofuran $(250 \mathrm{~mL})$ was then added via syringe. The stirred suspension was cooled to $0{ }^{\circ} \mathrm{C}$ and 4-pentenol $(6 \mathrm{~mL}, 58.1 \mathrm{mmol}, 1.0$ equiv) was added dropwise via syringe (caution: vigorous gas evolution). The cooling bath was removed, and the reaction was allowed to stir for $3 \mathrm{~h}$ at rt. The mixture was then recooled to $0^{\circ} \mathrm{C}$ and 1-bromobutane (12.5 mL, $116.2 \mathrm{mmol}, 2$ equiv) was added dropwise via syringe. The cooling bath was removed, and the mixture was stirred overnight at rt. The reaction was opened to air and quenched with saturated ammonium chloride $(50 \mathrm{~mL})$, adding the first few $\mathrm{mL}$ dropwise. This mixture was diluted with $200 \mathrm{~mL}$ diethyl ether, and the organic layer was separated, dried with $\mathrm{MgSO}_{4}$, filtered through Celite and concentrated in vacuo. The crude oil was purified by flash silica gel chromatography (5: 95 ethyl acetate : hexanes) affording $\mathbf{S 2}$ as a colorless oil $(70 \%)$ : ${ }^{1} \mathrm{H}$ NMR $\left(400 \mathrm{MHz}, \mathrm{CDCl}_{3}\right) \delta 5.82(\mathrm{ddt}, J=16.9,10.2,6.6 \mathrm{~Hz}, 1 \mathrm{H}), 5.02(\mathrm{dd}, J=17.1,1.9 \mathrm{~Hz}$, $1 \mathrm{H}), 4.95(\mathrm{dd}, J=10.2,2.0 \mathrm{~Hz}, 1 \mathrm{H}), 3.40(\mathrm{td}, J=6.6,2.8 \mathrm{~Hz}, 4 \mathrm{H}), 2.11(\mathrm{q}, J=6.9 \mathrm{~Hz}, 2 \mathrm{H}), 1.75-$ $1.62(\mathrm{~m}, 2 \mathrm{H}), 1.59-1.49(\mathrm{~m}, 2 \mathrm{H}), 1.43-1.31(\mathrm{~m}, 2 \mathrm{H}), 0.91(\mathrm{t}, J=7.4 \mathrm{~Hz}, 3 \mathrm{H}) ;{ }^{13} \mathrm{C}$ NMR $(101$ $\left.\mathrm{MHz}, \mathrm{CDCl}_{3}\right) \delta 138.5,114.8,70.8,70.3,32.0,30.5,29.1,19.5,14.1$; FTIR $\left(\mathrm{cm}^{-1}\right): 2954,2924$, 2854, 1457, 1377. HRMS $(\mathrm{Cl}) \mathrm{m} / \mathrm{z}$, calcd for $\left[\mathrm{C}_{9} \mathrm{H}_{19} \mathrm{O}\right]^{+}: 143.1436$; found: 143.1425 .<smiles>C=CCCCCCCCCCCCCC</smiles>

(S3) A $250 \mathrm{~mL}$ 3-neck round bottom flask equipped with a magnetic stir bar and a water condenser was charged with magnesium turnings (1.4 equiv), sealed with 3 rubber septa, flame dried under vacuum, cooled to rt and refilled with nitrogen. Anhydrous diethyl ether $(5 \mathrm{~mL})$ was added to cover the magnesium turnings and 5-bromopentene (1.2 equiv) was added dropwise via syringe. After the solution began to self-reflux the remaining diethyl ether $(95 \mathrm{~mL})$ and 5-bromopentene were slowly added as needed to maintain a gentle reflux. After all of the bromide was added, the reaction was heated to $40{ }^{\circ} \mathrm{C}$ to reflux for $1 \mathrm{~h}$. A separate oven dried round bottom flask equipped with a magnetic stir bar and rubber septum was cooled under vacuum, refilled with nitrogen, charged with dimethylphenylchlorosilane $(2.5 \mathrm{~mL}, 15 \mathrm{mmol}, 1.0$ equiv), diluted with anhydrous diethyl ether $(15 \mathrm{~mL}, 1 \mathrm{M})$ and cooled to $0{ }^{\circ} \mathrm{C}$. The Grignard reagent, prepared above, was added dropwise via syringe to the solution of dimethylphenylchlorosilane at $0{ }^{\circ} \mathrm{C}$. After the addition was complete, catalytic $\mathrm{ZnCl}_{2}(0.5 \mathrm{~mL}, 1 \mathrm{M}$ in diethyl ether, 0.03 equiv) was added via syringe and the reaction was stirred for $72 \mathrm{~h}$ at rt under nitrogen. The reaction was cooled to $0{ }^{\circ} \mathrm{C}$, opened to air, diluted with diethyl ether $(10 \mathrm{~mL})$ then slowly quenched with water $(15 \mathrm{~mL})$. The mixture was extracted with diethyl ether $(3 \times 10 \mathrm{~mL})$ and the combined organic layers were washed with brine, dried with $\mathrm{MgSO}_{4}$, filtered through Celite, and concentrated in vacuo. The crude oil was purified 
via flash silica gel chromatography (hexanes) to give $\mathbf{S 3}$ as a clear oil $(2.09 \mathrm{~g}, 68 \%):{ }^{1} \mathrm{H}$ NMR $\left(400 \mathrm{MHz}, \mathrm{CDCl}_{3}\right) \delta 7.56-7.49(\mathrm{~m}, 2 \mathrm{H}), 7.41-7.33(\mathrm{~m}, 3 \mathrm{H}), 5.79$ (ddt, $J=17.0,10.2,6.7 \mathrm{~Hz}$, $1 \mathrm{H}), 5.06-4.91(\mathrm{~m}, 2 \mathrm{H}), 2.08(\mathrm{q}, J=7.1 \mathrm{~Hz}, 2 \mathrm{H}), 1.51-1.36(\mathrm{~m}, 2 \mathrm{H}), 0.85-0.73(\mathrm{~m}, 2 \mathrm{H}), 0.28$ (s, $6 \mathrm{H}) ;{ }^{13} \mathrm{C} \mathrm{NMR}\left(101 \mathrm{MHz}, \mathrm{CDCl}_{3}\right) \delta 139.6,139.0,133.7,128.9,127.9,114.7,37.8,23.5,15.4$, 2.9; FTIR $\left(\mathrm{cm}^{-1}\right): 3069,2955,2922,1427,1248,1114,910,834,811,771,728,699,469$. HRMS (Cl) $\mathrm{m} / \mathrm{z}$, calcd for $\left[\mathrm{C}_{13} \mathrm{H}_{21} \mathrm{Si}^{+}: 205.1413\right.$; found: $205.1429{ }^{9}$

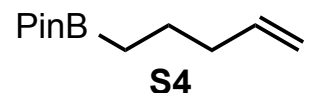

(S4) A $100 \mathrm{~mL}$ round bottom flask equipped with a magnetic stir bar was charged with 4-pentenyl-1-boronic acid (1.65 g, $14.5 \mathrm{mmol}, 1.0$ equiv), pinacol ( $1.75 \mathrm{~g}, 14.5 \mathrm{mmol}, 1.0$ equiv), $\mathrm{MgSO}_{4}$ ( $8.7 \mathrm{~g}, 5$ equiv) and diluted with diethyl ether $(15 \mathrm{~mL}, 1 \mathrm{M})$ under air. The reaction vessel was sealed with a rubber septum and stirred at rt overnight. The crude reaction mixture was filtered through Celite and concentrated in vacuo. The crude oil was purified via flash silica gel chromatography ( $5: 95$ ethyl acetate : hexanes) to give $\mathbf{S 4}$ as a colorless oil $(2.10 \mathrm{~g}, 75 \%):{ }^{1} \mathrm{H} \mathrm{NMR}\left(600 \mathrm{MHz}, \mathrm{CDCl}_{3}\right) \delta 5.80$ (ddt, $J=17.0,10.2,6.7 \mathrm{~Hz}, 1 \mathrm{H}), 4.99(\mathrm{dd}, 1 \mathrm{H}), 4.93(\mathrm{~d}, 1 \mathrm{H}), 2.05(\mathrm{q}, J=7.0 \mathrm{~Hz}, 2 \mathrm{H}), 1.51(\mathrm{p}, J=$ $7.7 \mathrm{~Hz}, 2 \mathrm{H}), 1.24(\mathrm{~s}, 12 \mathrm{H}), 0.79(\mathrm{t}, J=7.9 \mathrm{~Hz}, 2 \mathrm{H}) .{ }^{13} \mathrm{C}$ NMR $\left(151 \mathrm{MHz}, \mathrm{CDCl}_{3}\right) \delta 139.1,114.6$, 83.0, 36.5, 25.0, 23.6; ${ }^{11} \mathrm{~B}$ NMR (193 MHz, $\left.\mathrm{CDCl}_{3}\right) \delta 34.1$; FTIR $\left(\mathrm{cm}^{-1}\right): 2979,2931,1407,1378$, $1318,1146,969,910$. HRMS $(\mathrm{Cl}) \mathrm{m} / \mathrm{z}$, calcd for $\left[\mathrm{C}_{11} \mathrm{H}_{22} \mathrm{BO}_{2}\right]^{+}: 197.1713$; found: $197.1701 .^{10}$<smiles>C=CCCCC(C)(C)C(=O)OC</smiles>

(S5) A $100 \mathrm{~mL}$ round bottom flask equipped with a magnetic stir bar and a rubber septum was flame dried under vacuum, cooled to rt and refilled with nitrogen. Diisopropylamine $(3.08 \mathrm{~mL}, 24 \mathrm{mmol}, 1.2$ equiv) and anhydrous tetrahydrofuran $(10 \mathrm{~mL})$ were added via syringe and the mixture was cooled to $-78{ }^{\circ} \mathrm{C}$ under a positive pressure of nitrogen. $\mathrm{n}$-Butyllithium $(9.2 \mathrm{~mL}, 2.6 \mathrm{M}$ in hexanes, 1.95 equiv) was added dropwise via syringe and the solution was stirred for $1 \mathrm{~h}$ at $-78^{\circ} \mathrm{C}$. A solution of methyl isobutyrate $(2.3 \mathrm{~mL}$, $20 \mathrm{mmol}, 1.0$ equiv) in anhydrous tetrahydrofuran $(10 \mathrm{~mL}, 2 \mathrm{M})$ was added dropwise via syringe and the reaction was stirred for $2 \mathrm{~h}$ at $-78{ }^{\circ} \mathrm{C}$. 5-Bromopentene $(2.86 \mathrm{~mL}, 24 \mathrm{mmol}, 1.2$ equiv) was added via syringe then the cooling bath was removed and the reaction was stirred at it for 24 h. The reaction was then cooled to $0{ }^{\circ} \mathrm{C}$, opened to air and quenched with saturated aqueous ammonium chloride $(20 \mathrm{~mL})$. The mixture was extracted with diethyl ether $(3 \times 10 \mathrm{~mL})$ and the combined organic layers were washed with brine, dried with $\mathrm{MgSO}_{4}$, filtered through Celite, and concentrated in vacuo. The crude oil was purified via flash silica gel chromatography ( $5: 95$ ethyl acetate : hexanes) to give $\mathbf{S 5}$ as a colorless oil $(1.62 \mathrm{~g}, 48 \%):{ }^{1} \mathrm{H} \mathrm{NMR}\left(600 \mathrm{MHz}, \mathrm{CDCl}_{3}\right) \delta 5.78$ (ddt, $J=16.9,10.2,6.6 \mathrm{~Hz}, 1 \mathrm{H}), 4.99(\mathrm{dd}, J=17.1 \mathrm{~Hz}, 1 \mathrm{H}), 4.94(\mathrm{~d}, J=10.9 \mathrm{~Hz}, 1 \mathrm{H}), 3.65(\mathrm{~s}$, $3 \mathrm{H}), 2.02(\mathrm{q}, J=7.3 \mathrm{~Hz}, 2 \mathrm{H}), 1.55-1.47(\mathrm{~m}, 2 \mathrm{H}), 1.39-1.27(\mathrm{~m}, 2 \mathrm{H}), 1.16(\mathrm{~s}, 6 \mathrm{H}) ;{ }^{13} \mathrm{C} N M R$ $\left(151 \mathrm{MHz}, \mathrm{CDCl}_{3}\right) \delta 178.6,138.7,114.7,51.8,42.4,40.4,34.2,25.3,24.4 ; \mathrm{FTIR}\left(\mathrm{cm}^{-1}\right): 2978$, 2946, 1734, 1474, 1268, 1195, 1150, 911. HRMS $(\mathrm{Cl}) \mathrm{m} / \mathrm{z}$, calcd for $\left[\mathrm{C}_{10} \mathrm{H}_{19} \mathrm{O}_{2}\right]^{+}: 171.1385$; found: $171.1365 .^{11}$

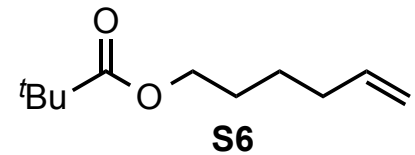

(S6) A $500 \mathrm{~mL}$ round bottom flask equipped with a magnetic stir bar and a rubber septum, was flame dried under vacuum, cooled to rt, and refilled with nitrogen. Anhydrous dichloromethane $(200 \mathrm{~mL}), 5-$ hexenol ( $4.8 \mathrm{~mL}, 40 \mathrm{mmol}, 1.0$ equiv), pivaloyl chloride $(5.9 \mathrm{~mL}, 48$ mmol, 1.2 equiv) and triethylamine $(6.7 \mathrm{~mL}, 48 \mathrm{mmol}, 1.2$ equiv) were each added sequentially via syringe. The reaction was stirred at $\mathrm{rt}$ for $20 \mathrm{~h}$. The reaction was then opened to air and quenched with $3 \mathrm{M}$ aqueous ammonium hydroxide $(60 \mathrm{~mL})$. The organic layer was separated then washed with an additional $3 \mathrm{M}$ ammonium hydroxide $(1 \times 60$ $\mathrm{mL})$ then with brine $(1 \times 60 \mathrm{~mL})$. The combined aqueous layers were extracted with dichloromethane $(1 \times 60 \mathrm{~mL})$. The combined organic layers were dried with $\mathrm{MgSO}_{4}$, filtered through Celite, and concentrated in vacuo. The crude liquid was purified via flash silica gel chromatography (5: 95 diethyl ether : hexanes) to give $\mathbf{S 6}$ as a colorless liquid $(6.57 \mathrm{~g}, 89 \%):{ }^{1} \mathrm{H}$ NMR $\left(400 \mathrm{MHz}, \mathrm{CDCl}_{3}\right) \delta 5.79$ (ddt, $\left.J=16.9,10.2,6.7 \mathrm{~Hz}, 1 \mathrm{H}\right), 5.01$ (dd, $J=17.1,1.6 \mathrm{~Hz}, 1 \mathrm{H}$ ), $4.96(\mathrm{dd}, J=10.2,3.1,1.2 \mathrm{~Hz}, 1 \mathrm{H}), 4.05(\mathrm{t}, J=6.6 \mathrm{~Hz}, 2 \mathrm{H}), 2.08(\mathrm{q}, J=7.2 \mathrm{~Hz}, 2 \mathrm{H}), 1.74-1.57$ $(\mathrm{m}, 2 \mathrm{H}), 1.54-1.37(\mathrm{~m}, 2 \mathrm{H}), 1.19(\mathrm{~s}, 9 \mathrm{H}) ;{ }^{13} \mathrm{C} \mathrm{NMR}\left(101 \mathrm{MHz}, \mathrm{CDCl}_{3}\right) \delta 178.8,138.6,114.9$, 
64.4, 38.9, 33.4, 28.2, 27.3, 25.3; FTIR $\left(\mathrm{cm}^{-1}\right): 2975,2936,1730,1481,1285,1156,911$. HRMS (Cl) $\mathrm{m} / \mathrm{z}$, calcd for $\left[\mathrm{C}_{11} \mathrm{H}_{21} \mathrm{O}_{2}\right]^{+}: 185.1542$; found: 185.1525 .

\section{Single Component Catalyst ((L1) $\left.{ }_{2} \mathrm{PdCl}_{2}\right)$ :}

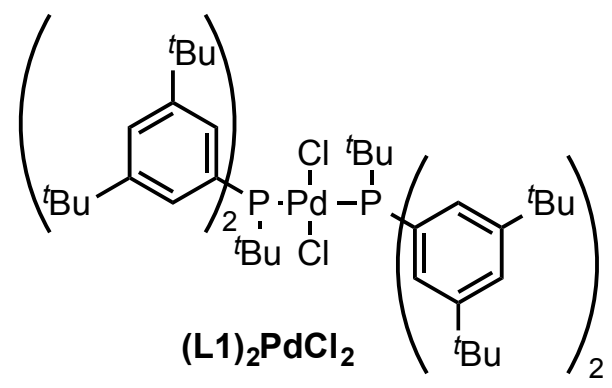

$\left((\mathbf{L} 1)_{2} \mathbf{P d C l}_{2}\right) \quad$ Bis(acetonitrile)dichloropalladium(II) (311 $\mathrm{mg}, \quad 1.2 \mathrm{mmol}, 1.0$ equiv) and bis(3,5-di-tertbutylphenyl)(tert-butyl)phosphine (1.12 g, $2.4 \mathrm{mmol}, 2.0$ equiv) was added to a $50 \mathrm{~mL}$ round bottom flask equipped with a magnetic stir bar and a rubber septum. The atmosphere in the flask was replaced with nitrogen, and anhydrous dichloromethane $(20 \mathrm{~mL})$ was added via syringe. The reaction was stirred for 30 minutes at rt. The flask was then briefly opened to air and the solvent was removed in vacuo ( 200 mtorr). The resulting solid was recrystallized from anhydrous diethyl ether $(15 \mathrm{~mL})$ under a nitrogen atmosphere at $0{ }^{\circ} \mathrm{C}$ for $24 \mathrm{~h}$ to give (L1) ${ }_{2} \mathrm{PdCl}_{2}$ as an air stable, fine yellow solid $\left(1.23 \mathrm{~g}, 92 \%\right.$ yield): ${ }^{1} \mathrm{H}$ NMR $(400 \mathrm{MHz}$, $\left.\mathrm{C}_{6} \mathrm{D}_{6}\right) \delta 8.08(\mathrm{t}, J=5.1 \mathrm{~Hz}, 8 \mathrm{H}), 7.60(\mathrm{~s}, 4 \mathrm{H}), 1.76(\mathrm{t}, J=7.3 \mathrm{~Hz}, 18 \mathrm{H}), 1.33(\mathrm{~s}, 72 \mathrm{H}) ;{ }^{13} \mathrm{C}$ NMR $\left(101 \mathrm{MHz}, \mathrm{C}_{6} \mathrm{D}_{6}\right) \delta 149.6(\mathrm{t}, J=4.7 \mathrm{~Hz}), 131.0(\mathrm{t}, J=5.8 \mathrm{~Hz}), 130.5(\mathrm{t}, J=40.1 \mathrm{~Hz}), 123.8,36.6$ $(\mathrm{t}, J=10.6 \mathrm{~Hz}), 35.2,31.6,31.1(\mathrm{t}, J=2.6 \mathrm{~Hz}) ;{ }^{31} \mathrm{P}$ NMR $\left(162 \mathrm{MHz}, \mathrm{C}_{6} \mathrm{D}_{6}\right) \delta 41.9 ; \mathrm{FTIR}\left(\mathrm{cm}^{-1}\right)$ : 2962, 2903, 2868, 1477, 1419, 1362, 1248, 1135, 708; $\mathrm{mp}=261^{\circ} \mathrm{C}$ (dec.). HRMS (LIFDI) $\mathrm{m} / \mathrm{z}$, calcd for $\left[\mathrm{C}_{64} \mathrm{H}_{102} \mathrm{Cl}_{2} \mathrm{P}_{2} \mathrm{Pd}\right.$ ]: 1108.5885; found: 1108.5583. Anal. Calcd for $\mathrm{C}_{64} \mathrm{H}_{102} \mathrm{Cl}_{2} \mathrm{P}_{2} \mathrm{Pd}$ : $\mathrm{C}$, 69.20; H, 9.26; Cl, 6.38. Found: C, 69.04; H, 9.38; Cl, 6.23.

A small portion of $(\mathbf{L} 1)_{2} \mathbf{P d C l}_{2}$ was dissolved in dichloromethane under air and recrystallized via slow evaporation at $\mathrm{rt}$ to give an X-ray quality crystal (Figure S1, see below for full crystallographic details).

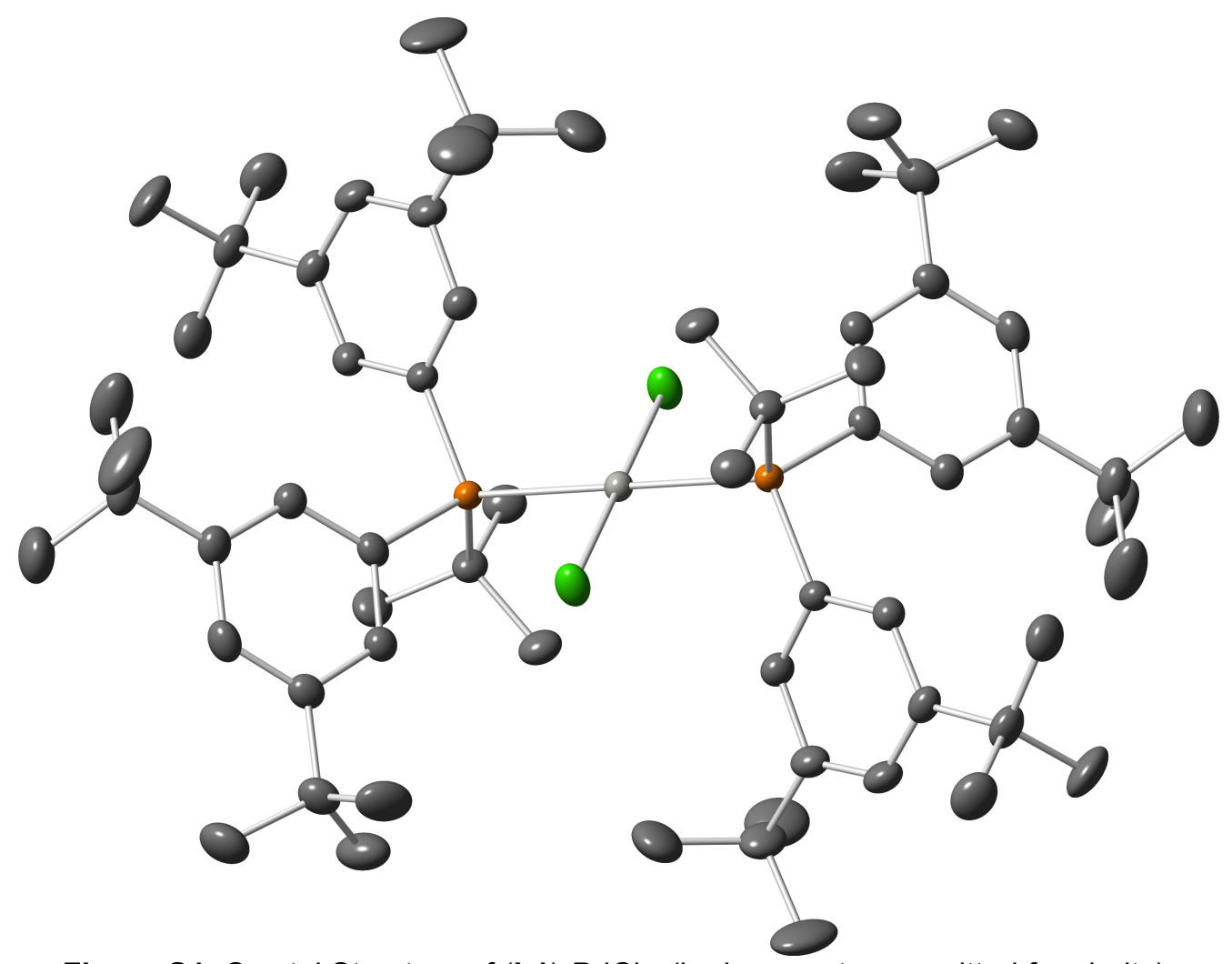

Figure S1. Crystal Structure of (L1) ${ }_{2} \mathrm{PdCl}_{2}$ (hydrogen atoms omitted for clarity). 


\section{Catecholchloroborane (catBCI):}

Note: Although catecholchloroborane is commercially available, for this communication we prepared it using a modification of literature procedures. ${ }^{12,13}$

Caution: This reaction generates 2 equivalents of anhydrous hydrogen chloride gas. The addition rate of boron trichloride must be controlled to prevent over-pressurization of the reaction vessel. All gas leaving the reaction should be scrubbed by base to prevent harm to people or equipment.<smiles>ClB1Oc2ccccc2O1</smiles>

(catBCl) A flame dried $500 \mathrm{~mL}$ Schlenk flask equipped with a magnetic stir bar and a rubber septum was cooled to rt under vacuum and refilled with nitrogen. The flask was briefly opened and quickly charged with catechol (10.46 g, $95 \mathrm{mmol}, 0.95$ equiv) then resealed. The flask was then evacuated and refilled with nitrogen 3 times. Anhydrous hexanes $(200 \mathrm{~mL})$ was added via canula transfer. With a positive flow of nitrogen, the septum was removed and the Schlenk flask was equipped with an oven dried $100 \mathrm{~mL}$ pressure-equalizing addition funnel equipped with a rubber septum. The apparatus was fitted with a vent tube attached to a gas scrubber filled with aqueous $\mathrm{NaOH}\left(250 \mathrm{~mL}, \sim 10 \mathrm{M}, 0{ }^{\circ} \mathrm{C}\right)$. The catechol solution was cooled to $0{ }^{\circ} \mathrm{C}$ with stirring $(\sim 1 \mathrm{~h})$. Boron trichloride $(100 \mathrm{~mL}, 1.0 \mathrm{M}$ in hexane, 1.0 equiv) was cannula transferred into the addition funnel (stopcock closed). The stopcock was opened slightly to add boron trichloride dropwise to the solution of catechol with rapid stirring. Once the addition of boron trichloride was complete, the reaction was allowed to warm to rt and stir overnight (8-12 h) with a slow, constant flow of nitrogen entering the Schlenk flask through the side arm, and exiting the addition funnel through the vent tube leading to the gas scrubber. After 8-12 h, when the solution has become homogeneous, the slow addition funnel was quickly replaced with a glass stopper and the hexanes was removed in vacuo ( 200 mtorr) through a solid $\mathrm{NaOH}$ trap to quench any residual hydrogen chloride gas. Once dried, the flask was sealed under vacuum, brought into a dry glovebox and scraped into vials. The catecholchloroborane was used without further purification, but it can be sublimed $\left(70{ }^{\circ} \mathrm{C}, 20\right.$ torr) to give a white solid $(13.77 \mathrm{~g}, 94 \%):{ }^{1} \mathrm{H}$ NMR (400 MHz, CDCl 3 ) $\delta 7.18-7.12(\mathrm{~m}, 2 \mathrm{H}), 7.07-7.00(\mathrm{~m}, 2 \mathrm{H}) ;{ }^{13} \mathrm{C}$ NMR $\left(101 \mathrm{MHz}, \mathrm{CDCl}_{3}\right) \delta$ 148.2, 123.6, 112.9; ${ }^{11} \mathrm{~B}$ NMR $\left(128 \mathrm{MHz}, \mathrm{CDCl}_{3}\right) \delta 28.9$.

\section{Comments and Preparation of Boric Acid Impregnated Silica:}

Note: Many boronic esters are known to partially decompose during purification on silica gel. However, boric acid impregnated silica has been shown to decrease this decomposition resulting in increased isolated yields. ${ }^{14}$ In our hands, use of commercial, untreated silica gel resulted in yields $\sim 5-10 \%$ lower than with boric acid impregnated silica gel (prepared according to the procedure below). Importantly, while boric acid impregnated silica gel is not necessary for this method, the use of it for purification did result in more consistent isolated yields.

Boric Acid Impregnated Silica: A $1000 \mathrm{~mL}$ round bottom flask equipped with a thick stir bar was charged with silica gel $(300 \mathrm{~mL})$, boric acid $(28 \mathrm{~g})$ and ethanol $(550 \mathrm{~mL})$ under air. The suspension was stirred at $\mathrm{rt}$ for $2 \mathrm{~h}$ at which time the ethanol and excess boric acid were removed by filtration through a fine glass frit. The impregnated silica was washed with ethanol $(3 \times 200 \mathrm{~mL})$ and left to dry on the frit under constant suction for $16 \mathrm{~h}$ then placed in a $140{ }^{\circ} \mathrm{C}$ gravity oven to dry for an additional $48 \mathrm{~h}$. Once dried, the boric acid impregnated silica was stored at $\mathrm{rt}$ in a desiccator until used. 


\section{General Procedure for the $\alpha$-Olefin Boryl-Heck Reaction:}

Note: All reactions in this section were performed on a $3 \mathrm{mmol}$ scale.

\section{General Procedure A ( $\alpha$-Olefins):}

In a nitrogen-filled glovebox, catecholchloroborane (1.5 equiv), lithium trifluoromethanesulfonate (1.5 equiv) and ( $\mathbf{L} 1)_{2} \mathrm{PdCl}_{2}(2.5 \mathrm{~mol} \%)$, were added to a flame-dried or oven-dried $25 \mathrm{~mL}$ Schlenk flask equipped with a stir bar. The flask was sealed with a rubber septum, removed from the glovebox and attached to a nitrogen manifold via the sidearm. $3 \mathrm{~mL}$ of trifluorotoluene $(1 \mathrm{~mL}$ per $\mathrm{mmol}$ ) was added via syringe with stirring. N,N-dicyclohexylmethylamine (5.0 equiv) was added via syringe and then the sides of the flask were rinsed with $3 \mathrm{~mL}$ of additional trifluorotoluene (1 $\mathrm{mL}$ per mmol). The solution was stirred in an oil bath at $90{ }^{\circ} \mathrm{C}$ for 15 minutes, at which time the alkene substrate (1.0 equiv) was added in one portion via syringe. The reaction was allowed to stir at $90{ }^{\circ} \mathrm{C}$ for $24 \mathrm{~h}$. After $24 \mathrm{~h}$, the reaction was removed from the oil bath and opened to air. Pinacol ( 3 equiv) was added in one portion and the reaction was removed from heat and stirred for $1 \mathrm{~h}$ at rt. At that time, and with the reactor now cooled to rt, the reaction was diluted with 20 $\mathrm{mL}$ of diethyl ether. The reaction was stirred for $10 \mathrm{~min}$, filtered through Celite and concentrated in vacuo to remove solvents, including trifluorotoluene. The crude oil was diluted with diethyl ether $(30 \mathrm{~mL})$ and washed with $1 \mathrm{M}$ hydrochloric acid $(3 \times 20 \mathrm{~mL})$ to remove excess amine. The organic layer was stirred with ammonium pyrrolidine-dithiocarbamate (palladium scavenger, 6 equiv to palladium $)^{15}$ for $1 \mathrm{~h}$, then dried with $\mathrm{MgSO}_{4}$, filtered through Celite and concentrated in vacuo. A small aliquot of the crude reaction mixture $(\sim 20 \mu \mathrm{L})$ was analyzed by ${ }^{1} \mathrm{H}$ NMR to determine the $E / Z$ ratio. The crude material was purified via silica column chromatography on boric acid impregnated silica gel (as prepared above) in the indicated solvent combination.

Note: Only the $E$ isomer is reported for the ${ }^{1} \mathrm{H}$ and ${ }^{13} \mathrm{C}$ NMR spectral data. The ${ }^{13} \mathrm{C}$ NMR spectra may contain extra, unassigned peaks, which are attributed to the minor $Z$ isomer.

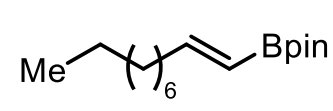

5

(5) According to general procedure A, catecholchloroborane (694 mg, 4.5 mmol), (L1) ${ }_{2} \mathrm{PdCl}_{2}$ (83 $\mathrm{mg}, 0.075 \mathrm{mmol}$ ), lithium trifluoromethanesulfonate (702 mg, $4.5 \mathrm{mmol}), \mathrm{N}, \mathrm{N}$-dicyclohexylmethylamine $(3.2 \mathrm{~mL}, 5.0 \mathrm{mmol})$, trifluorotoluene $(6.0 \mathrm{~mL}, 0.5 \mathrm{M})$, and 1-decene $(443 \mathrm{mg}, 3.0 \mathrm{mmol})$ were combined under $\mathrm{N}_{2}$ and stirred at $90{ }^{\circ} \mathrm{C}$ for $24 \mathrm{~h}$. The reaction was worked up according to general procedure $A$. Analysis of the crude reaction mixture via ${ }^{1} \mathrm{H}$ NMR revealed a $88: 12 \mathrm{E} / \mathrm{Z}$ ratio. The product was purified via boric acid impregnated silica gel chromatography $(5: 95$ dichloromethane : hexanes) to afford 5 as a colorless oil $(747 \mathrm{mg}, 94 \%)$ : ${ }^{1} \mathrm{H} \mathrm{NMR}(600 \mathrm{MHz}$, $\left.\mathrm{CDCl}_{3}\right) \delta 6.64(\mathrm{dt}, J=17.9,6.5 \mathrm{~Hz}, 1 \mathrm{H}), 5.42(\mathrm{dd}, J=17.9,1.9 \mathrm{~Hz}, 1 \mathrm{H}), 2.20-2.08(\mathrm{~m}, 2 \mathrm{H}), 1.45$ $-1.36(\mathrm{~m}, 2 \mathrm{H}), 1.30-1.22(\mathrm{~m}, 22 \mathrm{H}), 0.87(\mathrm{t}, 3 \mathrm{H}) ;{ }^{1} \mathrm{H}$ NMR $\left(400 \mathrm{MHz}, \mathrm{C}_{6} \mathrm{D}_{6}\right) \delta 7.00(\mathrm{dt}, J=17.8$, $6.5 \mathrm{~Hz}, 1 \mathrm{H}), 5.82(\mathrm{dt}, J=17.8,1.5 \mathrm{~Hz}, 1 \mathrm{H}), 2.07(\mathrm{q}, 2 \mathrm{H}), 1.42-1.13(\mathrm{~m}, 12 \mathrm{H}), 1.10(\mathrm{~s}, 12 \mathrm{H}), 0.92$ $-0.83(\mathrm{~m}, 3 \mathrm{H}) ;{ }^{13} \mathrm{C}$ NMR $\left(151 \mathrm{MHz}, \mathrm{CDCl}_{3}\right) \delta 155.0,83.1,36.0,32.0,29.6,29.4,28.4,24.9,22.8$, $14.3 ;{ }^{13} \mathrm{C}$ NMR $\left(101 \mathrm{MHz}, \mathrm{C}_{6} \mathrm{D}_{6}\right) \delta$ 155.1, 82.9, 36.3, 32.3, 29.9, 29.7, 29.6, 28.8, 25.0, 23.1, 14.4; ${ }^{11} \mathrm{~B}$ NMR $\left(193 \mathrm{MHz}, \mathrm{CDCl}_{3}\right) \delta 29.9 ;{ }^{11} \mathrm{~B}$ NMR $\left(128 \mathrm{MHz}, \mathrm{C}_{6} \mathrm{D}_{6}\right) \delta 30.0 ; \mathrm{FTIR}\left(\mathrm{cm}^{-1}\right): 2978,2926$, $2855,1639,1362,1320,1146$. HRMS $(\mathrm{Cl}) \mathrm{m} / \mathrm{z}$, calcd for $\left[\mathrm{C}_{16} \mathrm{H}_{32} \mathrm{BO}_{2}\right]^{+}:$267.2495; found: 267.2484.<smiles>Brc1ccc(Br)cc1</smiles>

(6) According to general procedure A, catecholchloroborane $(694 \mathrm{mg}$, $4.5 \mathrm{mmol}), \quad(\text { L1 })_{2} \mathrm{PdCl}_{2} \quad(83 \quad \mathrm{mg}, \quad 0.075 \mathrm{mmol})$, lithium trifluoromethanesulfonate $(702 \mathrm{mg}, \quad 4.5 \mathrm{mmol}), \quad \mathrm{N}, \mathrm{N}-$ dicyclohexylmethylamine $(3.2 \mathrm{~mL}, 5.0 \mathrm{mmol})$, trifluorotoluene $(6.0 \mathrm{~mL}$, $0.5 \mathrm{M}$ ), and 4-phenyl-1-butene (397 $\mathrm{mg}, 3.0 \mathrm{mmol}$ ) were combined under $\mathrm{N}_{2}$ and stirred at $90{ }^{\circ} \mathrm{C}$ for $24 \mathrm{~h}$. The reaction was worked up according to general procedure A. Analysis of the crude reaction mixture via ${ }^{1} \mathrm{H}$ NMR revealed a $88: 12 \mathrm{E} / \mathrm{Z}$ ratio. The product was purified via boric acid impregnated silica gel chromatography ( $3: 7$ dichloromethane : hexanes) to afford 6 as a colorless oil $(687 \mathrm{mg}, 89 \%):{ }^{1} \mathrm{H}$ NMR $\left(600 \mathrm{MHz}, \mathrm{CDCl}_{3}\right) \delta 7.30-7.27(\mathrm{~m}, 2 \mathrm{H}), 7.20-7.16(\mathrm{~m}$, 
$3 \mathrm{H}), 6.70(\mathrm{dt}, J=18.0,6.2 \mathrm{~Hz}, 1 \mathrm{H}), 5.50(\mathrm{~d}, J=18.0 \mathrm{~Hz}, 1 \mathrm{H}), 2.79-2.68(\mathrm{~m}, 2 \mathrm{H}), 2.53-2.39$ $(\mathrm{m}, 2 \mathrm{H}), 1.27(\mathrm{~s}, 12 \mathrm{H}) ;{ }^{1} \mathrm{H}$ NMR $\left(400 \mathrm{MHz}, \mathrm{C}_{6} \mathrm{D}_{6}\right) \delta 7.18-6.90(\mathrm{~m}, 6 \mathrm{H}), 5.78(\mathrm{~d}, J=17.9 \mathrm{~Hz}, 1 \mathrm{H})$, $2.57-2.48(\mathrm{~m}, 2 \mathrm{H}), 2.37-2.25(\mathrm{~m}, 2 \mathrm{H}), 1.09(\mathrm{~s}, 12 \mathrm{H}) ;{ }^{13} \mathrm{C} \mathrm{NMR}\left(101 \mathrm{MHz}, \mathrm{CDCl}_{3}\right) \delta 153.6$, 141.9, 128.5, 126.0, 83.2, 37.7, 34.7, 24.9; ${ }^{13} \mathrm{C}$ NMR $\left(101 \mathrm{MHz}, \mathrm{C}_{6} \mathrm{D}_{6}\right) \delta 153.8,141.9,128.7$, 128.6, 126.1, 83.0, 37.9, 35.0, 25.0; ${ }^{11} \mathrm{~B}$ NMR $\left(193 \mathrm{MHz}, \mathrm{CDCl}_{3}\right) \delta 29.7 ;{ }^{11} \mathrm{~B} \mathrm{NMR}(128 \mathrm{MHz}$, $\left.\mathrm{C}_{6} \mathrm{D}_{6}\right) \delta$ 29.8; FTIR $\left(\mathrm{cm}^{-1}\right): 2977,2929,1637,1361,1320,1144$. HRMS (Cl) m/z, calcd for $\left[\mathrm{C}_{16} \mathrm{H}_{24} \mathrm{BO}_{2}\right]^{+}:$259.1869; found: 259.1867 .<smiles>BrC(Br)=CC1CCCCC1</smiles>

(7) According to general procedure A, catecholchloroborane (694 mg, 4.5 mmol), (L1) ${ }_{2} \mathrm{PdCl}_{2}(83 \mathrm{mg}, 0.075 \mathrm{mmol}$ ), lithium trifluoromethanesulfonate (702 mg, $4.5 \mathrm{mmol}), \mathrm{N}, \mathrm{N}$-dicyclohexylmethylamine $(3.2 \mathrm{~mL}, 5.0 \mathrm{mmol})$, trifluorotoluene $(6.0 \mathrm{~mL}, 0.5 \mathrm{M})$, and vinylcyclohexane $(341 \mathrm{mg}, 3.0 \mathrm{mmol})$ were combined under $\mathrm{N}_{2}$ and stirred at $90{ }^{\circ} \mathrm{C}$ for $24 \mathrm{~h}$. The reaction was worked up according to general procedure A. Analysis of the crude reaction mixture via ${ }^{1} \mathrm{H}$ NMR revealed a $96: 4 \mathrm{E} / \mathrm{Z}$ ratio. The product was purified via boric acid impregnated silica gel chromatography $(1: 9$ dichloromethane : hexanes) to afford 7 as a pale yellow oil $(595 \mathrm{mg}, 84 \%):{ }^{1} \mathrm{H}$ NMR $(600 \mathrm{MHz}$, $\left.\mathrm{CDCl}_{3}\right) \delta 6.58(\mathrm{dd}, J=18.2,6.2 \mathrm{~Hz}, 1 \mathrm{H}), 5.38(\mathrm{~d}, J=18.2,1 \mathrm{H}), 2.07-1.94(\mathrm{~m}, 1 \mathrm{H}), 1.80-1.67$ $(\mathrm{m}, 4 \mathrm{H}), 1.67-1.60(\mathrm{~m}, 2 \mathrm{H}), 1.27(\mathrm{~s}, 12 \mathrm{H}), 1.20-1.00(\mathrm{~m}, 4 \mathrm{H}) ;{ }^{13} \mathrm{C}$ NMR $\left(151 \mathrm{MHz}, \mathrm{CDCl}_{3}\right) \delta$ 160.0, 83.1, 43.4, 32.1, 26.3, 26.1, 24.9; ${ }^{11} \mathrm{~B}$ NMR (193 MHz, $\left.\mathrm{CDCl}_{3}\right) \delta 30.1 ; \mathrm{FTIR}\left(\mathrm{cm}^{-1}\right): 2978$, $2925,2852,1636,1371,1349,1321,1147$. HRMS (Cl) $\mathrm{m} / \mathrm{z}$, calcd for $\left[\mathrm{C}_{14} \mathrm{H}_{26} \mathrm{BO}_{2}\right]^{+}: 237.2026$; found: 237.2041 .

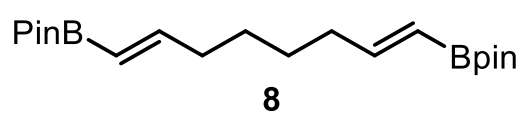

(8) According to general procedure $\mathrm{A}$, catecholchloroborane (694 mg, $4.5 \mathrm{mmol}),(\mathrm{L} 1)_{2} \mathrm{PdCl}_{2}$ (83 mg, $\left.0.075 \mathrm{mmol}\right)$, lithium trifluoromethanesulfonate $(702 \mathrm{mg}, 4.5 \mathrm{mmol}), \mathrm{N}, \mathrm{N}-$ dicyclohexylmethylamine $(3.2 \mathrm{~mL}, 5.0 \mathrm{mmol})$, trifluorotoluene $(6.0 \mathrm{~mL}, 0.5 \mathrm{M})$, and 1,7 -octadiene $(165 \mathrm{mg}, 1.5 \mathrm{mmol})$ were combined under $\mathrm{N}_{2}$ and stirred at 90 ${ }^{\circ} \mathrm{C}$ for $24 \mathrm{~h}$. The reaction was worked up according to general procedure $\mathrm{A}$. Analysis of the crude reaction mixture via ${ }^{1} \mathrm{H}$ NMR revealed a 90:10 E/Z ratio. The product was purified via boric acid impregnated silica gel chromatography (1:1 dichloromethane : hexanes) to afford 8 as a white solid (450 mg, 83\%): ${ }^{1} \mathrm{H}$ NMR $\left(600 \mathrm{MHz}, \mathrm{CDCl}_{3}\right) \delta 6.61(\mathrm{dt}, J=17.9,6.5 \mathrm{~Hz}, 2 \mathrm{H}), 5.42(\mathrm{~d}, J=$ $17.9 \mathrm{~Hz}, 2 \mathrm{H}), 2.20-2.07(\mathrm{~m}, 4 \mathrm{H}), 1.43(\mathrm{p}, J=3.5 \mathrm{~Hz}, 4 \mathrm{H}), 1.26(\mathrm{~s}, 24 \mathrm{H}) ;{ }^{13} \mathrm{C}$ NMR $(151 \mathrm{MHz}$, $\left.\mathrm{CDCl}_{3}\right) \delta 154.6,83.0,35.8,27.9,24.9 ;{ }^{11} \mathrm{~B}$ NMR $\left(193 \mathrm{MHz}, \mathrm{CDCl}_{3}\right) \delta 29.9 ; \mathrm{FTIR}\left(\mathrm{cm}^{-1}\right): 2978$, 2929, 1638, 1362, 1320, 1146; mp $=53{ }^{\circ} \mathrm{C}$. HRMS $(\mathrm{Cl}) \mathrm{m} / \mathrm{z}$, calcd for $\left[\mathrm{C}_{20} \mathrm{H}_{37} \mathrm{~B}_{2} \mathrm{O}_{4}\right]^{+}: 363.2878$; found: 363.2879 .

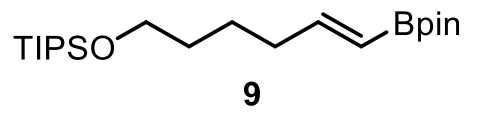

(9) According to general procedure $\mathrm{A}$, catecholchloroborane (694 mg, $4.5 \mathrm{mmol}$ ), (L1) ${ }_{2} \mathrm{PdCl}_{2}$ (83 $\mathrm{mg}, 0.075 \mathrm{mmol}$ ), lithium trifluoromethanesulfonate $(702 \mathrm{mg}, 4.5 \mathrm{mmol}), \quad \mathrm{N}, \mathrm{N}-$ dicyclohexylmethylamine $(3.2 \mathrm{~mL}, 5.0 \mathrm{mmol})$, trifluorotoluene (6.0 mL, $0.5 \mathrm{M})$, and triisopropylsiloxy-5-hexene $\left(770 \mathrm{mg}, 3.0 \mathrm{mmol}\right.$ ) were combined under $\mathrm{N}_{2}$ and stirred at $90{ }^{\circ} \mathrm{C}$ for $24 \mathrm{~h}$. The reaction was worked up according to general procedure. Analysis of the crude reaction mixture via ${ }^{1} \mathrm{H}$ NMR revealed a 90:10 E/Z ratio. The product was purified via boric acid impregnated silica gel chromatography ( $3: 7$ dichloromethane : hexanes) to afford 9 as a colorless oil $(1.02 \mathrm{~g}, 90 \%)$ : ${ }^{1} \mathrm{H}$ NMR $\left(600 \mathrm{MHz}, \mathrm{CDCl}_{3}\right) \delta 6.63(\mathrm{dt}, J=17.9,6.4 \mathrm{~Hz}$, $1 \mathrm{H}), 5.43(\mathrm{~d}, J=17.9 \mathrm{~Hz}, 1 \mathrm{H}), 3.67(\mathrm{t}, J=6.3 \mathrm{~Hz}, 2 \mathrm{H}), 2.17(\mathrm{q}, 2 \mathrm{H}), 1.67-1.43(\mathrm{~m}, 4 \mathrm{H}), 1.26(\mathrm{~s}$, $12 \mathrm{H}), 1.05(\mathrm{~s}, 21 \mathrm{H}) ;{ }^{13} \mathrm{C}$ NMR $\left(151 \mathrm{MHz}, \mathrm{CDCl}_{3}\right) \delta 154.7,83.1,63.3,35.8,32.7,24.9,24.7,18.2$, 12.2; ${ }^{11} \mathrm{~B}$ NMR $\left(193 \mathrm{MHz}, \mathrm{CDCl}_{3}\right) \delta$ 29.8; FTIR $\left(\mathrm{cm}^{-1}\right): 2941,2866,1638,1363,1320,1147,1108$. HRMS (Cl) m/z, calcd for $\left[\mathrm{C}_{21} \mathrm{H}_{44} \mathrm{BO}_{3} \mathrm{Si}\right]^{+}: 383.3153$; found: 383.3134 .

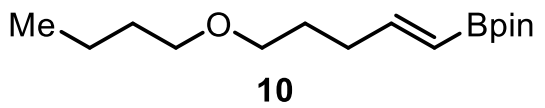

(10) According to general procedure $\mathrm{A}$, catecholchloroborane (694 mg, $4.5 \mathrm{mmol}),\left(\right.$ L1) ${ }_{2} \mathrm{PdCl}_{2}$ (83 $\mathrm{mg}, 0.075 \mathrm{mmol})$, lithium trifluoromethanesulfonate $(702 \mathrm{mg}$, $4.5 \mathrm{mmol}), \mathrm{N}, \mathrm{N}$-dicyclohexylmethylamine $(3.2 \mathrm{~mL}, 5.0 \mathrm{mmol})$, trifluorotoluene $(6.0 \mathrm{~mL}, 0.5 \mathrm{M})$, and 5-butoxy-pent-1-ene (426 mg, $3.0 \mathrm{mmol}$ ) were combined under $\mathrm{N}_{2}$ and stirred at $90{ }^{\circ} \mathrm{C}$ for 24 $\mathrm{h}$. The reaction was worked up according to general procedure A. Analysis of the crude reaction 
mixture via ${ }^{1} \mathrm{H}$ NMR revealed a $87: 13 \mathrm{E} / \mathrm{Z}$ ratio. The product was purified via boric acid impregnated silica gel chromatography (1:9 dichloromethane : hexanes) to afford 10 as a colorless oil (741 mg, 92\%): ${ }^{1} \mathrm{H}$ NMR $\left(600 \mathrm{MHz}, \mathrm{CDCl}_{3}\right) \delta 6.63(\mathrm{dt}, J=17.9,6.4 \mathrm{~Hz}, 1 \mathrm{H}), 5.45(\mathrm{~d}$, $J=18.0 \mathrm{~Hz}, 1 \mathrm{H}), 3.50-3.30(\mathrm{~m}, 4 \mathrm{H}), 2.28-2.13(\mathrm{~m}, 2 \mathrm{H}), 1.75-1.63(\mathrm{~m}, 2 \mathrm{H}), 1.54(\mathrm{ddd}, J=$ 14.6, 11.2, 4.9 Hz, 2H), $1.42-1.31(\mathrm{~m}, 2 \mathrm{H}), 1.26(\mathrm{~s}, 12 \mathrm{H}), 0.91(\mathrm{t}, J=7.4 \mathrm{~Hz}, 3 \mathrm{H}) ;{ }^{13} \mathrm{C}$ NMR $(151$ $\left.\mathrm{MHz}, \mathrm{CDCl}_{3}\right) \delta 154.0,83.2,70.8,70.3,32.5,32.0,28.4,24.9,19.5,14.1 ;{ }^{11} \mathrm{~B}$ NMR $(193 \mathrm{MHz}$, $\left.\mathrm{CDCl}_{3}\right) \delta$ 29.8; FTIR $\left(\mathrm{cm}^{-1}\right): 2977,2934,2862,1639,1364,1321,1146$. HRMS (Cl) m/z, calcd for $\left[\mathrm{C}_{15} \mathrm{H}_{30} \mathrm{BO}_{3}\right]^{+}:$269.2288; found: 269.2274 .<smiles>CC(C)(C=CBr)C=CBr</smiles>

11

(11) According to general procedure $\mathrm{A}$, catecholchloroborane $(694 \mathrm{mg}$, $4.5 \mathrm{mmol}), \quad(\mathbf{L} 1)_{2} \mathrm{PdCl}_{2} \quad(83 \mathrm{mg}, \quad 0.075 \mathrm{mmol}), \quad$ lithium trifluoromethanesulfonate $(702 \mathrm{mg}, \quad 4.5 \quad \mathrm{mmol}), \quad \mathrm{N}, \mathrm{N}-$ dicyclohexylmethylamine $(3.2 \mathrm{~mL}, 5.0 \mathrm{mmol})$, trifluorotoluene $(6.0 \mathrm{~mL}, 0.5$ $\mathrm{M})$, and 11-chloro-1-undecene (584 mg, $3.0 \mathrm{mmol}$ ) were combined under $\mathrm{N}_{2}$ and stirred at $90{ }^{\circ} \mathrm{C}$ for $24 \mathrm{~h}$. The reaction was worked up according to general procedure A. Analysis of the crude reaction mixture via ${ }^{1} \mathrm{H}$ NMR revealed a $88: 12 \mathrm{E} / \mathrm{Z}$ ratio. The product was purified via boric acid impregnated silica gel chromatography (1:4 dichloromethane : hexanes) to afford 11 as a colorless oil (830 mg, 88\%): ${ }^{1} \mathrm{H}$ NMR $\left(600 \mathrm{MHz}, \mathrm{CDCl}_{3}\right) \delta 6.63(\mathrm{dt}, J=18.0,6.4 \mathrm{~Hz}, 1 \mathrm{H}), 5.42(\mathrm{~d}$, $J=18.0,1 \mathrm{H}), 3.53(\mathrm{t}, J=6.8 \mathrm{~Hz}, 2 \mathrm{H}), 2.19-2.03(\mathrm{~m}, 2 \mathrm{H}), 1.76(\mathrm{p}, 2 \mathrm{H}), 1.46-1.36(\mathrm{~m}, 4 \mathrm{H}), 1.28$ (s, 8H), $1.26(\mathrm{~s}, 12 \mathrm{H}) ;{ }^{1} \mathrm{H}$ NMR $\left(400 \mathrm{MHz}, \mathrm{C}_{6} \mathrm{D}_{6}\right) \delta 7.01(\mathrm{dt}, J=17.8,6.5 \mathrm{~Hz}, 1 \mathrm{H}), 5.83(\mathrm{~d}, J=$ $17.8 \mathrm{~Hz}, 1 \mathrm{H}), 3.12(\mathrm{t}, J=6.7 \mathrm{~Hz}, 2 \mathrm{H}), 2.09(\mathrm{q}, J=6.6 \mathrm{~Hz}, 2 \mathrm{H}), 1.56-1.23(\mathrm{~m}, 6 \mathrm{H}), 1.21-0.97$ $(\mathrm{m}, 2 \mathrm{OH}) ;{ }^{13} \mathrm{C}$ NMR $\left(151 \mathrm{MHz}, \mathrm{CDCl}_{3}\right) \delta 154.9,83.1,45.3,36.0,32.8,29.5,29.3,29.0,28.3,27.0$, 25.0; ${ }^{13} \mathrm{C}$ NMR $\left(101 \mathrm{MHz}, \mathrm{C}_{6} \mathrm{D}_{6}\right) \delta 154.9,83.0,45.0,36.2,32.9,29.71,29.65,29.5,29.1,28.8$, 27.1, 25.0; ${ }^{11} \mathrm{~B}$ NMR $\left(193 \mathrm{MHz}, \mathrm{CDCl}_{3}\right) \delta 30.0 ;{ }^{11} \mathrm{~B}$ NMR $\left(128 \mathrm{MHz}, \mathrm{C}_{6} \mathrm{D}_{6}\right) \delta 29.3 ; \mathrm{FTIR}\left(\mathrm{cm}^{-1}\right)$ : $2978,2928,2855,1639,1362,1330,1146$. HRMS $(\mathrm{Cl}) \mathrm{m} / \mathrm{z}$, calcd for $\left[\mathrm{C}_{17} \mathrm{H}_{33} \mathrm{BO}_{2} \mathrm{Cl}\right]^{+}: 315.2262$; found: 315.2263 .

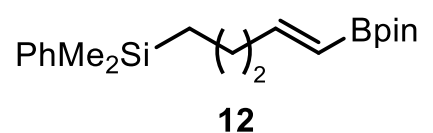

(12) According to general procedure A, catecholchloroborane (694 $\mathrm{mg}, \quad 4.5 \mathrm{mmol}),(\mathbf{L 1})_{2} \mathrm{PdCl}_{2}(83 \mathrm{mg}, \quad 0.075 \mathrm{mmol})$, lithium trifluoromethanesulfonate $(702 \mathrm{mg}, \quad 4.5 \mathrm{mmol}), \quad \mathrm{N}, \mathrm{N}-$ dicyclohexylmethylamine $(3.2 \mathrm{~mL}, 5.0 \mathrm{mmol})$, trifluorotoluene $(6.0$ $\mathrm{mL}, 0.5 \mathrm{M}$ ), and (dimethyl)pent-4-enyl(phenyl)silane $\left(613 \mathrm{mg}, 3.0 \mathrm{mmol}\right.$ ) were combined under $\mathrm{N}_{2}$ and stirred at $90{ }^{\circ} \mathrm{C}$ for $24 \mathrm{~h}$. The reaction was worked up according to general procedure $\mathrm{A}$. Analysis of the crude reaction mixture via ${ }^{1} \mathrm{H}$ NMR revealed a 89:11 $\mathrm{E} / \mathrm{Z}$ ratio. The product was purified via boric acid impregnated silica gel chromatography (1:9 dichloromethane : hexanes) to afford 12 as a yellow oil $(881 \mathrm{mg}, 89 \%)$ : ${ }^{1} \mathrm{H}$ NMR $\left(400 \mathrm{MHz}, \mathrm{CDCl}_{3}\right) \delta 7.59-7.44(\mathrm{~m}, 2 \mathrm{H}), 7.40-$ $7.31(\mathrm{~m}, 3 \mathrm{H}), 6.60(\mathrm{dt}, J=17.9,6.5 \mathrm{~Hz}, 1 \mathrm{H}), 5.46-5.37(\mathrm{~m}, 1 \mathrm{H}), 2.24-2.09(\mathrm{~m}, 2 \mathrm{H}), 1.54-1.36$ $(\mathrm{m}, 2 \mathrm{H}), 1.26(\mathrm{~s}, 12 \mathrm{H}), 0.82-0.71(\mathrm{~m}, 2 \mathrm{H}), 0.25(\mathrm{~s}, 6 \mathrm{H}) ;{ }^{13} \mathrm{C}$ NMR $\left(101 \mathrm{MHz}, \mathrm{CDCl}_{3}\right) \delta 154.6$, 139.5, 133.7, 128.9, 127.8, 83.1, 39.8, 24.9, 22.9, 15.6, -2.9; ${ }^{11} \mathrm{~B}$ NMR $\left(193 \mathrm{MHz}, \mathrm{CDCl}_{3}\right) \delta 29.8$; FTIR $\left(\mathrm{cm}^{-1}\right)$ : 2978, 2924, 1638, 1427, 1398, 1389, 1362, 1320, 1249, 1214, 1146, 972, 850, 835, 812, 729, 700. HRMS (Cl) m/z, calcd for $\left[\mathrm{C}_{19} \mathrm{H}_{32} \mathrm{BO}_{2} \mathrm{Si}\right]^{+}: 331.2265$; found: 331.2245 .

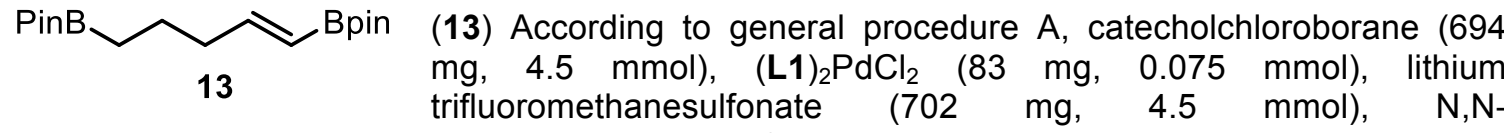
dicyclohexylmethylamine $(3.2 \mathrm{~mL}, 5.0 \mathrm{mmol})$, trifluorotoluene $(6.0 \mathrm{~mL}, 0.5 \mathrm{M})$, and pent-4-enylpinicolborane $\left(570 \mathrm{mg}, 2.9 \mathrm{mmol}\right.$ ) were combined under $\mathrm{N}_{2}$ and stirred at $90{ }^{\circ} \mathrm{C}$ for $24 \mathrm{~h}$. The reaction was worked up according to general procedure $A$. Analysis of the crude reaction mixture via ${ }^{1} \mathrm{H}$ NMR revealed a 89:11 E/Z ratio. The product was purified via boric acid impregnated silica gel chromatography (1:9 dichloromethane : hexanes) to afford 13 as a yellow oil $(677 \mathrm{mg}, 72 \%)$ : ${ }^{1} \mathrm{H}$ NMR $\left(400 \mathrm{MHz}, \mathrm{CDCl}_{3}\right) \delta 6.59(\mathrm{dt}, J=17.9,6.5 \mathrm{~Hz}, 1 \mathrm{H}), 5.39(\mathrm{~d}, J=17.9 \mathrm{~Hz}, 1 \mathrm{H}), 2.25-2.01$ $(\mathrm{m}, 2 \mathrm{H}), 1.68-1.38(\mathrm{~m}, 2 \mathrm{H}), 1.23(\mathrm{~s}, 12 \mathrm{H}), 1.21(\mathrm{~s}, 12 \mathrm{H}), 0.76(\mathrm{t}, J=7.9 \mathrm{~Hz}, 2 \mathrm{H}) ;{ }^{\mathrm{H}} \mathrm{NMR}(400$ $\left.\mathrm{MHz}, \mathrm{CD}_{3} \mathrm{CN}\right) \delta 6.52(\mathrm{dt}, J=17.9,6.6 \mathrm{~Hz}, 1 \mathrm{H}), 5.35(\mathrm{dt}, J=17.9,1.5 \mathrm{~Hz}, 1 \mathrm{H}), 2.15-2.09(\mathrm{~m}$, 2H), $1.47(\mathrm{p}, J=7.6 \mathrm{~Hz}, 2 \mathrm{H}), 1.21(\mathrm{~s}, 12 \mathrm{H}), 1.20(\mathrm{~s}, 12 \mathrm{H}), 0.70(\mathrm{t}, J=7.7 \mathrm{~Hz}, 2 \mathrm{H}) ;{ }^{13} \mathrm{C}$ NMR $(101$ $\left.\mathrm{MHz}, \mathrm{CDCl}_{3}\right) \delta 154.7,83.1,83.0,38.5,25.0,22.9 ;{ }^{13} \mathrm{C} \mathrm{NMR}\left(101 \mathrm{MHz}, \mathrm{CD}_{3} \mathrm{CN}\right) \delta 155.2,83.8$, 83.7, 38.8, 25.12, 25.07, 23.6; ${ }^{11} \mathrm{~B}$ NMR $\left(193 \mathrm{MHz}, \mathrm{CDCl}_{3}\right) \delta 34.1,29.9 ;{ }^{11} \mathrm{~B} \mathrm{NMR}(128 \mathrm{MHz}$, 
$\left.\mathrm{CD}_{3} \mathrm{CN}\right) \delta 35.5,31.2 ; \mathrm{FTIR}\left(\mathrm{cm}^{-1}\right): 2978,2931,1631,1371,1362,1319,1267,1239,1214,1146$, 969, 849; HRMS $(\mathrm{Cl}) \mathrm{m} / \mathrm{z}$, calcd for $\left[\mathrm{C}_{17} \mathrm{H}_{33} \mathrm{~B}_{2} \mathrm{O}_{4}\right]^{+}: 323.2565$; found: 323.2576.<smiles>COC(=O)C(C)(C)CCC/C=C/Cc1ccccc1</smiles>

(16) According to general procedure $A$, catecholchloroborane (694 mg, $4.5 \mathrm{mmol}$ ), (L1) ${ }_{2} \mathrm{PdCl}_{2}$ (83 $\mathrm{mg}, 0.075 \mathrm{mmol}$ ), lithium trifluoromethanesulfonate $(702 \mathrm{mg}, 4.5 \mathrm{mmol}), \mathrm{N}, \mathrm{N}-$ dicyclohexylmethylamine $(3.2 \mathrm{~mL}, 5.0 \mathrm{mmol})$, trifluorotoluene $(6.0 \mathrm{~mL}, 0.5 \mathrm{M})$, and methyl 2,2-dimethylhept-6-enoate $(511 \mathrm{mg}$, $3.0 \mathrm{mmol}$ ) were combined under $\mathrm{N}_{2}$ and stirred at $90{ }^{\circ} \mathrm{C}$ for 24 h. The reaction was worked up according to general procedure A. Analysis of the crude reaction mixture via ${ }^{1} \mathrm{H}$ NMR revealed a 87:13 E/Z ratio. The product was purified via boric acid impregnated silica gel chromatography ( $7: 3$ dichloromethane : hexanes) to afford 16 as a yellow oil $(732 \mathrm{mg}, 82 \%)$ : ${ }^{1} \mathrm{H}$ NMR $\left(400 \mathrm{MHz}, \mathrm{CDCl}_{3}\right) \delta 6.60(\mathrm{dt}, J=18.0,6.4 \mathrm{~Hz}, 1 \mathrm{H}), 5.42(\mathrm{dt}, J=18.0$, $1.6 \mathrm{~Hz}, 1 \mathrm{H}), 3.65(\mathrm{~s}, 3 \mathrm{H}), 2.17-1.99(\mathrm{~m}, 2 \mathrm{H}), 1.55-1.46(\mathrm{~m}, 2 \mathrm{H}), 1.34$ (tdd, J = 8.9, 7.1, $4.5 \mathrm{~Hz}$, $2 \mathrm{H}), 1.26(\mathrm{~s}, 12 \mathrm{H}), 1.15(\mathrm{~s}, 6 \mathrm{H}) ;{ }^{13} \mathrm{C}$ NMR $\left(101 \mathrm{MHz}, \mathrm{CDCl}_{3}\right) \delta 178.6,154.2,83.2,51.8,42.4$, 40.5, 36.3, 25.3, 24.9, 23.8; ${ }^{11} \mathrm{~B}$ NMR $\left(193 \mathrm{MHz}, \mathrm{CDCl}_{3}\right) \delta 30.2 ; \mathrm{FTIR}\left(\mathrm{cm}^{-1}\right): 2978,2944,1733$, 1639, 1362, 1320, 1146. HRMS (Cl) m/z, calcd for $\left[\mathrm{C}_{16} \mathrm{H}_{30} \mathrm{BO}_{4}\right]^{+}: 297.2237$; found: 297.2240.<smiles>O=C(Br)COCCCC/C=C/[Pb]</smiles>

(17) According to general procedure $A$, catecholchloroborane (694 mg, $4.5 \mathrm{mmol}$ ), (L1) ${ }_{2} \mathrm{PdCl}_{2}$ (83 mg, $\left.0.075 \mathrm{mmol}\right)$, lithium trifluoromethanesulfonate $(702 \mathrm{mg}, \quad 4.5 \mathrm{mmol}), \quad \mathrm{N}, \mathrm{N}-$ dicyclohexylmethylamine $(3.2 \mathrm{~mL}, 5.0 \mathrm{mmol})$, trifluorotoluene $(6.0 \mathrm{~mL}, 0.5 \mathrm{M})$, and hex-5-enyl pivalate $(553 \mathrm{mg}, 3.0 \mathrm{mmol})$ were combined under $\mathrm{N}_{2}$ and stirred at $90{ }^{\circ} \mathrm{C}$ for $24 \mathrm{~h}$. The reaction was worked up according to general procedure $A$. Analysis of the crude reaction mixture via ${ }^{1} \mathrm{H}$ NMR revealed a $88: 12 \mathrm{E} / \mathrm{Z}$ ratio. The product was purified via boric acid impregnated silica gel chromatography $(3: 7$ dichloromethane : hexanes) to afford 17 as a yellow oil (847 mg, 91\%): ${ }^{1} \mathrm{H} \mathrm{NMR}(600 \mathrm{MHz}$, $\left.\mathrm{CDCl}_{3}\right) \delta 6.61(\mathrm{dt}, J=18.0,6.4 \mathrm{~Hz}, 1 \mathrm{H}), 5.44(\mathrm{~d}, J=18.0 \mathrm{~Hz}, 1 \mathrm{H}), 4.04(\mathrm{t}, J=6.5 \mathrm{~Hz}, 2 \mathrm{H}), 2.19$ (tdd, $J=7.7,6.3,1.5 \mathrm{~Hz}, 2 \mathrm{H}), 1.68-1.59(\mathrm{~m}, 2 \mathrm{H}), 1.53-1.44(\mathrm{~m}, 2 \mathrm{H}), 1.26(\mathrm{~s}, 12 \mathrm{H}), 1.19(\mathrm{~s}$, 9H); ${ }^{13} \mathrm{C}$ NMR $\left(101 \mathrm{MHz}, \mathrm{CDCl}_{3}\right) \delta 178.8,154.0,83.2,64.3,38.9,35.4,28.3,27.3,24.9,24.7 ;{ }^{11} \mathrm{~B}$ NMR (193 MHz, $\left.\mathrm{CDCl}_{3}\right) \delta$ 29.8; FTIR $\left(\mathrm{cm}^{-1}\right): 2978,2935,1730,1269,1363,1147$. HRMS (Cl) $\mathrm{m} / \mathrm{z}$, calcd for $\left[\mathrm{C}_{17} \mathrm{H}_{32} \mathrm{BO}_{4}\right]^{+}: 311.2394$; found: 311.2379 .

(18) According to general procedure $\mathrm{A}$, catecholchloroborane $(694 \mathrm{mg}$,<smiles>C(=C/Cc1ccccc1)\Cc1ccccc1</smiles>
$4.5 \mathrm{mmol}), \quad(\mathbf{L} 1)_{2} \mathrm{PdCl}_{2} \quad(83 \quad \mathrm{mg}, \quad 0.075 \quad \mathrm{mmol}), \quad$ lithium trifluoromethanesulfonate $(702 \quad \mathrm{mg}, \quad 4.5 \quad \mathrm{mmol}), \quad \mathrm{N}, \mathrm{N}-$ dicyclohexylmethylamine $(3.2 \mathrm{~mL}, 5.0 \mathrm{mmol})$, trifluorotoluene $(6.0 \mathrm{~mL}, 0.5$ $\mathrm{M})$, and allylbenzene (356 mg, $3.0 \mathrm{mmol}$ ) were combined under $\mathrm{N}_{2}$ and stirred at $90^{\circ} \mathrm{C}$ for $24 \mathrm{~h}$. The reaction was worked up according to general procedure $A$. The product was purified via boric acid impregnated silica gel chromatography $(3: 7$ dichloromethane : hexanes) to afford four isomers of 18 as a yellow oil ( $527 \mathrm{mg}, 72 \%):{ }^{1} \mathrm{H}$ NMR $\left(400 \mathrm{MHz}, \mathrm{CDCl}_{3}\right) E$-allyl product: $\delta 7.35-$ $7.14(\mathrm{~m}, 5 \mathrm{H}), 6.38(\mathrm{~d}, J=15.8 \mathrm{~Hz}, 1 \mathrm{H}), 6.30(\mathrm{~m}, 1 \mathrm{H}), 1.88(\mathrm{~d}, J=7.0 \mathrm{~Hz}, 2 \mathrm{H}), 1.26(\mathrm{~s}, 12 \mathrm{H}),{ }^{11} \mathrm{~B}$ NMR $\left(128 \mathrm{MHz}, \mathrm{CDCl}_{3}\right) \delta 33.6$; E-alkenyl product: $\delta 7.35-7.14(\mathrm{~m}, 5 \mathrm{H}), 6.76(\mathrm{dt}, J=17.8,6.3$ $\mathrm{Hz}, 1 \mathrm{H}), 5.45(\mathrm{dt}, J=17.9,1.6 \mathrm{~Hz}, 1 \mathrm{H}), 3.48(\mathrm{dd}, J=6.3,1.4 \mathrm{~Hz}, 2 \mathrm{H}), 1.25(\mathrm{~s}, 12 \mathrm{H}) ;{ }^{11} \mathrm{~B}$ NMR $\left(128 \mathrm{MHz}, \mathrm{CDCl}_{3}\right) \delta 30.4$; FTIR $\left(\mathrm{cm}^{-1}\right): 3026,2978,2931,1637,1496,1449,1361,1325,1271$, 1214, 1167, 1144, 1109, 998, 967, 887, 851, 745, 695, 673. HRMS (Cl) m/z, calcd for $\left[\mathrm{C}_{15} \mathrm{H}_{22} \mathrm{BO}_{2}\right]^{+}:$245.1713; found: 245.1722 . 


\section{General Procedure for the Styrene Boryl-Heck Reaction:}

Note: All reactions in this section were performed on a $3 \mathrm{mmol}$ scale.

\section{General Procedure B (Styrenes):}

In a nitrogen-filled glovebox, catecholchloroborane (1.5 equiv) was added to a dry $25 \mathrm{~mL}$ Schlenk flask equipped with a stir bar. The flask was sealed with a rubber septum, removed from the glovebox and attached to a nitrogen manifold via the sidearm. The flask was briefly opened and lithium iodide $(5 \mathrm{~mol} \%)$ and $(\mathbf{L} 1)_{2} \mathrm{PdCl}_{2}(2.5 \mathrm{~mol} \%)$ were added and the flask was resealed, then evacuated and refilled with nitrogen 3 times. $3 \mathrm{~mL}$ of trifluorotoluene $(1 \mathrm{~mL}$ per mmol) was added via syringe with stirring. $\mathrm{N}, \mathrm{N}$-dicyclohexylmethylamine (5.0 equiv) was added via syringe and then the sides of the flask were rinsed with $3 \mathrm{~mL}$ of additional trifluorotoluene $(1 \mathrm{~mL}$ per mmol). The solution was stirred in an oil bath at $70{ }^{\circ} \mathrm{C}$ for 15 minutes, at which time the alkene substrate $(1.0$ equiv) was added in one portion via syringe. The reaction was allowed to stir at $70^{\circ} \mathrm{C}$ for $24 \mathrm{~h}$. After $24 \mathrm{~h}$, the reaction was removed from the oil bath and opened to air. Pinacol (3 equiv) was added in one portion and the reaction was removed from heat and stirred for $1 \mathrm{~h}$ at $\mathrm{rt}$. At that time, and with the reactor now cooled to rt, the reaction was diluted with $20 \mathrm{~mL}$ of diethyl ether. The reaction was stirred for $10 \mathrm{~min}$, filtered through Celite and concentrated in vacuo to remove solvents, including trifluorotoluene. The crude oil was diluted with diethyl ether $(30 \mathrm{~mL})$ and washed with $1 \mathrm{M}$ hydrochloric acid $(3 \times 20 \mathrm{~mL})$ to remove excess amine. The organic layer was stirred with ammonium pyrrolidine-dithiocarbamate (palladium scavenger, 6 equiv to palladium) ${ }^{15}$ for $1 \mathrm{~h}$, then dried with $\mathrm{MgSO}_{4}$, filtered through Celite and concentrated in vacuo. The crude material was purified via silica column chromatography on boric acid impregnated silica gel (as prepared above) in the indicated solvent combination.<smiles>CC(C)(C)c1ccc(C=Cc2ccccc2)cc1</smiles>

(19) According to general procedure $\mathrm{B}$, catecholchloroborane $(694 \mathrm{mg}$, $4.5 \mathrm{mmol}$ ), (L1) ${ }_{2} \mathrm{PdCl}_{2}$ (83 $\mathrm{mg}, 0.075 \mathrm{mmol}$ ), lithium iodide $(20 \mathrm{mg}, 0.15$ $\mathrm{mmol}), \quad \mathrm{N}, \mathrm{N}$-dicyclohexylmethylamine $(3.2 \mathrm{~mL}, \quad 5.0 \mathrm{mmol})$, trifluorotoluene $(6.0 \mathrm{~mL}, 0.5 \mathrm{M})$, and 4-tert-butylstyrene $(481 \mathrm{mg}, 3.0$ mmol) were combined under $\mathrm{N}_{2}$ and stirred at $70{ }^{\circ} \mathrm{C}$ for $24 \mathrm{~h}$. The reaction was worked up according to general procedure $\mathrm{B}$ and purified on boric acid impregnated silica gel chromatography ( $3: 7$ dichloromethane : hexanes) to afford 19 as a white solid (814 $\mathrm{mg}, 95 \%)$ : ${ }^{1} \mathrm{H}$ NMR $\left(600 \mathrm{MHz}, \mathrm{CDCl}_{3}\right) \delta 7.43(\mathrm{~d}, J=8.3 \mathrm{~Hz}, 2 \mathrm{H}), 7.41-7.33(\mathrm{~m}, 3 \mathrm{H}), 6.12(\mathrm{~d}, J=18.4 \mathrm{~Hz}$, $1 \mathrm{H}), 1.31(\mathrm{~s}, 21 \mathrm{H}) ;{ }^{13} \mathrm{C}$ NMR $\left(151 \mathrm{MHz}, \mathrm{CDCl}_{3}\right) \delta 152.3,149.5,134.9,127.0,125.7,83.4,34.9$, 31.4, 25.0; ${ }^{11} \mathrm{~B}$ NMR $\left(193 \mathrm{MHz}, \mathrm{CDCl}_{3}\right) \delta 30.6$; FTIR $\left(\mathrm{cm}^{-1}\right): 2966,1625,1346,1329,1141 ; \mathrm{mp}=$ 89-90 ${ }^{\circ} \mathrm{C}$. HRMS $(\mathrm{Cl}) \mathrm{m} / \mathrm{z}$, calcd for $\left[\mathrm{C}_{18} \mathrm{H}_{28} \mathrm{BO}_{2}\right]^{+}: 287.2182$; found: 287.2191 .<smiles>Cc1ccc(/C=C/[Ba]c2ccccc2)c(C)c1</smiles>

(20) According to general procedure $\mathrm{B}$, catecholchloroborane $(694 \mathrm{mg}$, $4.5 \mathrm{mmol}$ ), (L1) $)_{2} \mathrm{PdCl}_{2}$ (83 $\mathrm{mg}, 0.075 \mathrm{mmol}$ ), lithium iodide (20 $\mathrm{mg}, 0.15$ $\mathrm{mmol}), \quad \mathrm{N}, \mathrm{N}$-dicyclohexylmethylamine $(3.2 \mathrm{~mL}, \quad 5.0 \mathrm{mmol})$, trifluorotoluene $(6.0 \mathrm{~mL}, 0.5 \mathrm{M})$, and 2,4-dimethylstyrene (408 mg, 3.0 mmol) were combined under $\mathrm{N}_{2}$ and stirred at $70{ }^{\circ} \mathrm{C}$ for $24 \mathrm{~h}$. The reaction was worked up according to general procedure $\mathrm{B}$ and purified on boric acid impregnated silica gel chromatography (7:13 dichloromethane : hexanes) to afford 20 as a pale yellow oil $(742 \mathrm{mg}$, 99\%): ${ }^{1} \mathrm{H}$ NMR $\left(600 \mathrm{MHz}, \mathrm{CDCl}_{3}\right) \delta 7.62(\mathrm{~d}, J=18.3 \mathrm{~Hz}, 1 \mathrm{H}), 7.47(\mathrm{~d}, J=7.9 \mathrm{~Hz}, 1 \mathrm{H}), 6.99(\mathrm{~d}, J$ $=8.0 \mathrm{~Hz}, 1 \mathrm{H}), 6.97(\mathrm{~s}, 1 \mathrm{H}), 6.04(\mathrm{~d}, J=18.3 \mathrm{~Hz}, 1 \mathrm{H}), 2.39(\mathrm{~s}, 3 \mathrm{H}), 2.30(\mathrm{~s}, 3 \mathrm{H}), 1.31(\mathrm{~s}, 12 \mathrm{H}) ;{ }^{13} \mathrm{C}$ NMR $\left(151 \mathrm{MHz}, \mathrm{CDCl}_{3}\right) \delta 147.1,138.7,136.4,134.0,131.3,127.0,125.9,83.4,25.0,21.3,19.9$; ${ }^{11} \mathrm{~B}$ NMR $\left(193 \mathrm{MHz}, \mathrm{CDCl}_{3}\right) \delta 29.6$; FTIR $\left(\mathrm{cm}^{-1}\right): 2978,1622,1348,1323,1145 . \mathrm{HRMS}(\mathrm{Cl}) \mathrm{m} / \mathrm{z}$, calcd for $\left[\mathrm{C}_{16} \mathrm{H}_{24} \mathrm{BO}_{2}\right]^{+}:$259.1869; found: 259.1876 .<smiles>COc1ccc(/C=C/[Ba]c2ccccc2)cc1</smiles>

(21) According to general procedure $B$, catecholchloroborane $(694$ $\mathrm{mg}, 4.5 \mathrm{mmol}),(\mathbf{L} 1)_{2} \mathrm{PdCl}_{2}(83 \mathrm{mg}, 0.075 \mathrm{mmol})$, lithium iodide $(20$ $\mathrm{mg}, 0.15 \mathrm{mmol}), \mathrm{N}, \mathrm{N}$-dicyclohexylmethylamine $(3.2 \mathrm{~mL}, 5.0 \mathrm{mmol})$, trifluorotoluene $(6.0 \mathrm{~mL}, 0.5 \mathrm{M})$, and 4-vinylanisole $(403 \mathrm{mg}, 3.0$ 
mmol) were combined under $\mathrm{N}_{2}$ and stirred at $70{ }^{\circ} \mathrm{C}$ for $24 \mathrm{~h}$. The reaction was worked up according to general procedure $\mathrm{B}$ and purified on boric acid impregnated silica gel chromatography (1: 1 dichloromethane : hexanes) to afford 21 as a yellow solid $(765 \mathrm{mg}, 98 \%)$ : ${ }^{1} \mathrm{H}$ NMR $\left(600 \mathrm{MHz}, \mathrm{CDCl}_{3}\right) \delta 7.44(\mathrm{~d}, J=8.6 \mathrm{~Hz}, 2 \mathrm{H}), 7.35(\mathrm{~d}, J=18.4 \mathrm{~Hz}, 1 \mathrm{H}), 6.87(\mathrm{~d}, J=8.7$ $\mathrm{Hz}, 2 \mathrm{H}), 6.01(\mathrm{~d}, J=18.4 \mathrm{~Hz}, 1 \mathrm{H}), 3.81(\mathrm{~s}, 3 \mathrm{H}), 1.31(\mathrm{~s}, 12 \mathrm{H}) ;{ }^{13} \mathrm{C} \mathrm{NMR}\left(151 \mathrm{MHz}, \mathrm{CDCl}_{3}\right) \delta$ $160.4,149.2,130.6,128.6,114.1,83.4,55.4,25.0 ;{ }^{11} \mathrm{~B}$ NMR $\left(193 \mathrm{MHz}, \mathrm{CDCl}_{3}\right) \delta 30.3 ; \mathrm{FTIR}\left(\mathrm{cm}^{-}\right.$ $\left.{ }^{1}\right): 2978,1626,1605,1511,1355,1254,1144 ; \mathrm{mp}=55{ }^{\circ} \mathrm{C}$. HRMS (Cl) $\mathrm{m} / \mathrm{z}$, calcd for $\left[\mathrm{C}_{15} \mathrm{H}_{22} \mathrm{BO}_{3}\right]^{+}: 261.1662$; found: 261.1652 .<smiles>CN(C)c1ccc(/C=C/c2ccccc2)cc1</smiles>

(22) According to general procedure $B$, catecholchloroborane (694 $\mathrm{mg}, 4.5 \mathrm{mmol}$ ), (L1) ${ }_{2} \mathrm{PdCl}_{2}$ (83 $\mathrm{mg}, 0.075 \mathrm{mmol}$ ), lithium iodide (20 $\mathrm{mg}, 0.15 \mathrm{mmol}), \mathrm{N}, \mathrm{N}$-dicyclohexylmethylamine $(3.2 \mathrm{~mL}, 5.0 \mathrm{mmol})$, trifluorotoluene $(6.0 \mathrm{~mL}, \quad 0.5 \mathrm{M})$, and $\mathrm{N}, \mathrm{N}$-dimethylamino-4vinylbenzene $\left(442 \mathrm{mg}, 3.0 \mathrm{mmol}\right.$ ) were combined under $\mathrm{N}_{2}$ and stirred at $70{ }^{\circ} \mathrm{C}$ for $24 \mathrm{~h}$. The reaction was worked up according to general procedure $\mathrm{B}$ and purified on boric acid impregnated silica gel chromatography (1:9 dichloromethane : hexanes) to afford 22 as a pale yellow solid (766 mg, 94\%): ${ }^{1} \mathrm{H}$ NMR $\left(400 \mathrm{MHz}, \mathrm{CDCl}_{3}\right) \delta 7.40(\mathrm{~d}, J=8.8 \mathrm{~Hz}, 2 \mathrm{H}), 7.33(\mathrm{~d}, J=18.4 \mathrm{~Hz}, 1 \mathrm{H})$, $6.66(\mathrm{~d}, J=8.8 \mathrm{~Hz}, 2 \mathrm{H}), 5.92(\mathrm{~d}, J=18.3 \mathrm{~Hz}, 1 \mathrm{H}), 2.98(\mathrm{~s}, 6 \mathrm{H}), 1.30(\mathrm{~s}, 12 \mathrm{H}) ;{ }^{13} \mathrm{C}$ NMR $(151$ $\left.\mathrm{MHz}, \mathrm{CDCl}_{3}\right) \delta 151.1,149.9,128.5,126.1,112.1,83.2,40.5,25.0 ;{ }^{11} \mathrm{~B}$ NMR $\left(193 \mathrm{MHz}, \mathrm{CDCl}_{3}\right) \delta$ 29.8; FTIR $\left(\mathrm{cm}^{-1}\right): 2977,1604,1522,1430,1388,1351,1319,1227,1197,1182,1166,1143$, $807 ; \mathrm{mp}=83-87^{\circ} \mathrm{C}$. HRMS (ESI) m/z, calcd for $\left[\mathrm{C}_{16} \mathrm{H}_{25} \mathrm{BNO}_{2}\right]^{+}: 274.1973$; found: 274.1984 .<smiles>COc1cccc(/C=C/Cc2ccccc2)c1</smiles>

(23) According to general procedure $B$, catecholchloroborane (694 $\mathrm{mg}, 4.5 \mathrm{mmol}),(\mathbf{L} 1)_{2} \mathrm{PdCl}_{2}(83 \mathrm{mg}, 0.075 \mathrm{mmol})$, lithium iodide $(20$ $\mathrm{mg}, 0.15 \mathrm{mmol}), \mathrm{N}, \mathrm{N}$-dicyclohexylmethylamine $(3.2 \mathrm{~mL}, 5.0 \mathrm{mmol})$, trifluorotoluene $(6.0 \mathrm{~mL}, 0.5 \mathrm{M})$, and 3-vinylanisole $(403 \mathrm{mg}, 3.0$ mmol) were combined under $\mathrm{N}_{2}$ and stirred at $70{ }^{\circ} \mathrm{C}$ for $24 \mathrm{~h}$. The reaction was worked up according to general procedure $\mathrm{B}$ and purified on boric acid impregnated silica gel chromatography (2: 98 ethyl acetate : hexanes) to afford 23 as a yellow oil $(657 \mathrm{mg}, 84 \%):{ }^{1} \mathrm{H}$ NMR $\left(600 \mathrm{MHz}, \mathrm{CDCl}_{3}\right) \delta 7.37(\mathrm{~d}, J=18.4 \mathrm{~Hz}, 1 \mathrm{H}), 7.28-7.22(\mathrm{~m}, 1 \mathrm{H}), 7.08(\mathrm{~d}, J=7.6 \mathrm{~Hz}, 1 \mathrm{H})$, $7.03(\mathrm{~s}, 1 \mathrm{H}), 6.85(\mathrm{dd}, J=8.2,2.6 \mathrm{~Hz}, 1 \mathrm{H}), 6.16(\mathrm{~d}, J=18.4 \mathrm{~Hz}, 1 \mathrm{H}), 3.81(\mathrm{~s}, 3 \mathrm{H}), 1.31(\mathrm{~s}, 12 \mathrm{H})$; ${ }^{13} \mathrm{C}$ NMR $\left(151 \mathrm{MHz}, \mathrm{CDCl}_{3}\right) \delta 159.9,149.5,139.1,129.7,120.0,115.0,112.1,83.5,55.3,25.0$; ${ }^{11} \mathrm{~B}$ NMR $\left(193 \mathrm{MHz}, \mathrm{CDCl}_{3}\right) \delta 30.3$; FTIR $\left(\mathrm{cm}^{-1}\right): 2977,1625,1350,1260,1145$. HRMS (Cl) m/z, calcd for $\left[\mathrm{C}_{15} \mathrm{H}_{22} \mathrm{BO}_{3}\right]^{+}: 261.1662$; found: 261.1644 .<smiles>CCOC(=O)c1ccc(/C=C/c2ccccc2)cc1</smiles>

(24) According to general procedure $\mathrm{B}$, catecholchloroborane (694 $\mathrm{mg}, 4.5 \mathrm{mmol}),(\mathrm{L} 1)_{2} \mathrm{PdCl}_{2}$ (83 $\left.\mathrm{mg}, 0.075 \mathrm{mmol}\right)$, lithium iodide $(20$ $\mathrm{mg}, 0.15 \mathrm{mmol}), \mathrm{N}, \mathrm{N}$-dicyclohexylmethylamine $(3.2 \mathrm{~mL}, 5.0 \mathrm{mmol})$, trifluorotoluene $(6.0 \mathrm{~mL}, 0.5 \mathrm{M})$, and ethyl 4-vinylbenzoate $(529 \mathrm{mg}$, $3.0 \mathrm{mmol}$ ) were combined under $\mathrm{N}_{2}$ and stirred at $70{ }^{\circ} \mathrm{C}$ for $24 \mathrm{~h}$. The reaction was worked up according to general procedure $\mathrm{B}$ and purified on boric acid impregnated silica gel chromatography (1:1 dichloromethane : hexanes) to afford 24 as a pale yellow solid $(778 \mathrm{mg}$, $86 \%):{ }^{1} \mathrm{H}$ NMR $\left(600 \mathrm{MHz}, \mathrm{CDCl}_{3}\right) \delta 8.01(\mathrm{~d}, J=8.3 \mathrm{~Hz}, 2 \mathrm{H}), 7.53(\mathrm{~d}, J=8.2 \mathrm{~Hz}, 2 \mathrm{H}), 7.41(\mathrm{~d}, J=$ $18.4 \mathrm{~Hz}, 1 \mathrm{H}), 6.27(\mathrm{~d}, J=18.4 \mathrm{~Hz}, 1 \mathrm{H}), 4.37(\mathrm{q}, J=7.1 \mathrm{~Hz}, 2 \mathrm{H}), 1.39(\mathrm{t}, J=7.1 \mathrm{~Hz}, 3 \mathrm{H}), 1.32(\mathrm{~s}$, $12 \mathrm{H}) ;{ }^{13} \mathrm{C}$ NMR $\left(151 \mathrm{MHz}, \mathrm{CDCl}_{3}\right) \delta 166.4,148.3,141.8,130.7,130.0,127.0,83.7,61.1,25.0$, 14.5; ${ }^{11} \mathrm{~B}$ NMR (193 MHz, $\left.\mathrm{CDCl}_{3}\right) \delta 30.2 ; \mathrm{FTIR}\left(\mathrm{cm}^{-1}\right): 2979,1717,1624,1414,1381,1370,1350$, $1327,1273,1213,1176,1144,1106,970,850,764 ; \mathrm{mp}=79-80^{\circ} \mathrm{C}$. HRMS (Cl) $\mathrm{m} / \mathrm{z}$, calcd for $\left[\mathrm{C}_{17} \mathrm{H}_{24} \mathrm{BO}_{4}\right]^{+}:$303.1768; found: 303.1771 .

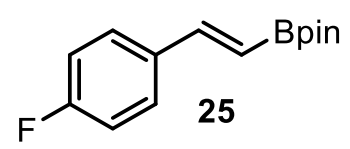

(25) According to general procedure B, catecholchloroborane $(694 \mathrm{mg}$, $4.5 \mathrm{mmol}$ ), (L1) ${ }_{2} \mathrm{PdCl}_{2}$ (83 $\mathrm{mg}, 0.075 \mathrm{mmol}$ ), lithium iodide $(20 \mathrm{mg}, 0.15$ $\mathrm{mmol}), \mathrm{N}, \mathrm{N}$-dicyclohexylmethylamine $(3.2 \mathrm{~mL}, 5.0 \mathrm{mmol})$, trifluorotoluene $(6.0 \mathrm{~mL}, 0.5 \mathrm{M})$, and 4-fluorostyrene $(386 \mathrm{mg}, 3.0 \mathrm{mmol})$ were combined under $\mathrm{N}_{2}$ and stirred at $70{ }^{\circ} \mathrm{C}$ for $24 \mathrm{~h}$. The reaction was worked up according to general procedure $\mathrm{B}$ and purified on boric acid impregnated silica gel chromatography (1: 4 dichloromethane : hexanes) to afford 25 as a white solid (582 mg, 78\%): ${ }^{1} \mathrm{H} \mathrm{NMR}(600 \mathrm{MHz}$, 
$\left.\mathrm{CDCl}_{3}\right) \delta 7.51-7.42(\mathrm{~m}, 2 \mathrm{H}), 7.35(\mathrm{~d}, J=18.4 \mathrm{~Hz}, 1 \mathrm{H}), 7.02(\mathrm{t}, J=8.6 \mathrm{~Hz}, 2 \mathrm{H}), 6.07$ (d, $J=18.4$ $\mathrm{Hz}, 1 \mathrm{H}), 1.31(\mathrm{~s}, 12 \mathrm{H}) ;{ }^{13} \mathrm{C}$ NMR $\left(101 \mathrm{MHz}, \mathrm{CDCl}_{3}\right) \delta 163.3(\mathrm{~d}, J=250 \mathrm{~Hz}), 148.3,133.8(\mathrm{~d}, J=3$ $\mathrm{Hz}), 128.8(\mathrm{~d}, J=8 \mathrm{~Hz}), 115.7(\mathrm{~d}, J=22 \mathrm{~Hz}), 83.5,25.0 ;{ }^{11} \mathrm{~B}$ NMR $\left(193 \mathrm{MHz}, \mathrm{CDCl}_{3}\right) \delta 30.3 ;{ }^{19} \mathrm{~F}$ NMR $\left(565 \mathrm{MHz}, \mathrm{CDCl}_{3}\right) \delta-112.4 ; \mathrm{FTIR}\left(\mathrm{cm}^{-1}\right): 2978,1508,1350,1326,1144 ; \mathrm{mp}=62-63{ }^{\circ} \mathrm{C}$. HRMS $(\mathrm{Cl}) \mathrm{m} / \mathrm{z}$, calcd for $\left[\mathrm{C}_{14} \mathrm{H}_{19} \mathrm{BO}_{2} \mathrm{~F}\right]^{+}:$249.1462; found: 249.1470 .<smiles>Clc1ccc(/C=C/c2ccccc2)cc1</smiles>

(26) According to general procedure B, catecholchloroborane (694 mg, $4.5 \mathrm{mmol}),(\mathrm{L} 1)_{2} \mathrm{PdCl}_{2}$ (83 $\left.\mathrm{mg}, 0.075 \mathrm{mmol}\right)$, lithium iodide $(20 \mathrm{mg}, 0.15$ $\mathrm{mmol})$, N,N-dicyclohexylmethylamine $(3.2 \mathrm{~mL}, 5.0 \mathrm{mmol})$, trifluorotoluene $(6.0 \mathrm{~mL}, 0.5 \mathrm{M})$, and 4-chlorostyrene $(416 \mathrm{mg}, 3.0$ mmol) were combined under $\mathrm{N}_{2}$ and stirred at $70{ }^{\circ} \mathrm{C}$ for $24 \mathrm{~h}$. The reaction was worked up according to general procedure $\mathrm{B}$ and purified on boric acid impregnated silica gel chromatography ( $3: 7$ dichloromethane : hexanes) to afford 26 as a white solid $(745 \mathrm{mg}, 94 \%)$ : ${ }^{1} \mathrm{H}$ NMR $\left(600 \mathrm{MHz}, \mathrm{CDCl}_{3}\right) \delta 7.41(\mathrm{~d}, 2 \mathrm{H}), 7.33(\mathrm{~d}, J=18.5 \mathrm{~Hz}, 1 \mathrm{H}), 7.30(\mathrm{~d}, 2 \mathrm{H}), 6.13(\mathrm{~d}, J=$ $18.4 \mathrm{~Hz}, 1 \mathrm{H}), 1.31(\mathrm{~s}, 12 \mathrm{H}) ;{ }^{13} \mathrm{C}$ NMR $\left(151 \mathrm{MHz}, \mathrm{CDCl}_{3}\right) \delta 148.2,136.1,134.8,129.0,128.4$, 83.6, 25.0; ${ }^{1} \mathrm{~B}$ NMR (193 MHz, $\left.\mathrm{CDCl}_{3}\right) \delta 30.2 ; \mathrm{FTIR}\left(\mathrm{cm}^{-1}\right)$ : 2974, 1627, 1492, 1411, 1380, 1372 , $1356,1323,1212,1166,1146,1089,993,803,640,491 ; \mathrm{mp}=84-85^{\circ} \mathrm{C}$. HRMS (Cl) m/z, calcd for $\left[\mathrm{C}_{14} \mathrm{H}_{19} \mathrm{BO}_{2} \mathrm{Cl}\right]^{+}: 265.1167$; found: 265.1170 .

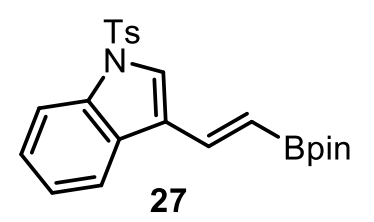

(27) According to general procedure $\mathrm{B}$, catecholchloroborane $(694 \mathrm{mg}$, $4.5 \mathrm{mmol}$ ), (L1) ${ }_{2} \mathrm{PdCl}_{2}$ (83 $\mathrm{mg}, 0.075 \mathrm{mmol}$ ), lithium iodide $(20 \mathrm{mg}, 0.15$ $\mathrm{mmol}), \mathrm{N}, \mathrm{N}$-dicyclohexylmethylamine $(3.2 \mathrm{~mL}, 5.0 \mathrm{mmol})$, trifluorotoluene $(6.0 \mathrm{~mL}, 0.5 \mathrm{M})$, and 1-(toluene-4-sulfonyl)-2-vinyl-1H-pyrrole $(893 \mathrm{mg}$, $3.0 \mathrm{mmol}$ ) were combined under $\mathrm{N}_{2}$ and stirred at $70{ }^{\circ} \mathrm{C}$ for $24 \mathrm{~h}$. The reaction was worked up according to general procedure $B$ and purified on boric acid impregnated silica gel chromatography ( $3: 2$ dichloromethane : hexanes) to afford 27 as a white solid (906 mg, 71\%): ${ }^{1} \mathrm{H}$ NMR $\left(600 \mathrm{MHz}, \mathrm{CDCl}_{3}\right) \delta 7.98(\mathrm{~d}, J=8.5 \mathrm{~Hz}, 1 \mathrm{H}), 7.86(\mathrm{~d}$, $J=8.1 \mathrm{~Hz}, 1 \mathrm{H}), 7.75(\mathrm{~d}, J=8.5 \mathrm{~Hz}, 2 \mathrm{H}), 7.69(\mathrm{~s}, 1 \mathrm{H}), 7.46(\mathrm{~d}, J=18.7 \mathrm{~Hz}, 1 \mathrm{H}), 7.37-7.30(\mathrm{~m}$, 1H), $7.26(\mathrm{~s}, 1 \mathrm{H}), 7.21(\mathrm{~d}, J=8.2 \mathrm{~Hz}, 2 \mathrm{H}), 6.20(\mathrm{~d}, J=18.7 \mathrm{~Hz}, 1 \mathrm{H}), 2.33(\mathrm{~s}, 3 \mathrm{H}), 1.32(\mathrm{~s}, 12 \mathrm{H})$; ${ }^{13} \mathrm{C}$ NMR $\left(101 \mathrm{MHz}, \mathrm{CDCl}_{3}\right) \delta 145.3,140.4,135.8,135.0,130.1,128.9,127.0,126.4,125.2$, 123.9, 121.6, 121.0, 113.8, 83.6, 25.0, 21.7; ${ }^{11} \mathrm{~B}$ NMR (193 MHz, $\left.\mathrm{CDCl}_{3}\right) \delta 29.2 ; \mathrm{FTIR}\left(\mathrm{cm}^{-1}\right)$ : 2977, 1627, 1372, 1348, 1175, 1142, 972; $\mathrm{mp}=69{ }^{\circ} \mathrm{C}$. HRMS (ESI) $\mathrm{m} / \mathrm{z}$, calcd for $\left[\mathrm{C}_{23} \mathrm{H}_{27} \mathrm{BNO}_{2} \mathrm{~S}\right]^{+}:$424.1748; found: 424.1765 .<smiles>BrC(Br)=Cc1ccc2c(c1)OCO2</smiles>

(28) According to general procedure $\mathrm{B}$, catecholchloroborane $(694 \mathrm{mg}$, $4.5 \mathrm{mmol}$ ), (L1) $)_{2} \mathrm{PdCl}_{2}$ (83 $\left.\mathrm{mg}, 0.075 \mathrm{mmol}\right)$, lithium iodide $(20 \mathrm{mg}, 0.15$ $\mathrm{mmol}), \quad \mathrm{N}, \mathrm{N}$-dicyclohexylmethylamine $(3.2 \mathrm{~mL}, \quad 5.0 \mathrm{mmol})$, trifluorotoluene $(6.0 \mathrm{~mL}, 0.5 \mathrm{M})$, and ethenyl-1,3-benzodioxole $(445 \mathrm{mg}$, $3.0 \mathrm{mmol}$ ) were combined under $\mathrm{N}_{2}$ and stirred at $70{ }^{\circ} \mathrm{C}$ for $24 \mathrm{~h}$. The reaction was worked up according to general procedure $\mathrm{B}$ and purified on boric acid impregnated silica gel chromatography ( $2: 3$ dichloromethane : hexanes) to afford 28 as a yellow solid $(616 \mathrm{mg}, 76 \%)$ : ${ }^{1} \mathrm{H} \mathrm{NMR}\left(600 \mathrm{MHz}, \mathrm{CDCl}_{3}\right) \delta 7.30(\mathrm{~d}, J=18.3 \mathrm{~Hz}, 1 \mathrm{H}), 7.03(\mathrm{~s}, 1 \mathrm{H}), 6.94(\mathrm{~d}, J=9.3 \mathrm{~Hz}, 1 \mathrm{H}), 6.77$ $(\mathrm{d}, J=8.0 \mathrm{~Hz}, 1 \mathrm{H}), 5.99-5.93(\mathrm{~m}, 3 \mathrm{H}), 1.31(\mathrm{~s}, 12 \mathrm{H}) ;{ }^{13} \mathrm{C} \mathrm{NMR}\left(151 \mathrm{MHz}, \mathrm{CDCl}_{3}\right) \delta 149.2$, 148.5, 148.2, 132.4, 122.8, 108.4, 106.0, 101.3, 83.4, 25.0; ${ }^{11} \mathrm{~B}$ NMR $\left(193 \mathrm{MHz}, \mathrm{CDCl}_{3}\right) \delta 30.4$; FTIR $\left(\mathrm{cm}^{-1}\right): 2978,1506,1446,1330,1250,1144,1040 ; \mathrm{mp}=83^{\circ} \mathrm{C}$. HRMS (Cl) $\mathrm{m} / \mathrm{z}$, calcd for $\left[\mathrm{C}_{15} \mathrm{H}_{20} \mathrm{BO}_{4}\right]^{+}:$275.1455; found: 275.1456.<smiles>CC(=Cc1ccccc1)c1ccccc1</smiles>

(29) According to general procedure $B$, catecholchloroborane (694 mg, 4.5 $\mathrm{mmol}),(\mathbf{L} 1)_{2} \mathrm{PdCl}_{2}(83 \mathrm{mg}, 0.075 \mathrm{mmol})$, lithium iodide $(20 \mathrm{mg}, 0.15 \mathrm{mmol})$, $\mathrm{N}, \mathrm{N}$-dicyclohexylmethylamine $(3.2 \mathrm{~mL}, 5.0 \mathrm{mmol})$, trifluorotoluene $(6.0 \mathrm{~mL}$, $0.5 \mathrm{M}$ ), and a-methylstyrene (354 $\mathrm{mg}, 3.0 \mathrm{mmol}$ ) were combined under $\mathrm{N}_{2}$ and stirred at $70{ }^{\circ} \mathrm{C}$ for $24 \mathrm{~h}$. The reaction was worked up according to general procedure $\mathrm{B}$ and purified on boric acid impregnated silica gel chromatography $(7: 13$ dichloromethane : hexanes) to afford 29 as a light orange oil $(517 \mathrm{mg}, 71 \%)$ : ${ }^{1} \mathrm{H} \mathrm{NMR}(600 \mathrm{MHz}$, $\left.\mathrm{CDCl}_{3}\right) \delta 7.50(\mathrm{~d}, 2 \mathrm{H}), 7.32(\mathrm{t}, J=7.4 \mathrm{~Hz}, 2 \mathrm{H}), 7.28(\mathrm{~d}, 1 \mathrm{H}), 5.76(\mathrm{~s}, 1 \mathrm{H}), 2.41(\mathrm{~s}, 3 \mathrm{H}), 1.32(\mathrm{~s}$, $12 \mathrm{H}) ;{ }^{13} \mathrm{C}$ NMR $\left(151 \mathrm{MHz}, \mathrm{CDCl}_{3}\right) \delta 157.9,144.0,128.3,128.1,126.0,83.1,25.1,20.2 ;{ }^{11} \mathrm{~B}$ NMR 
(193 MHz, $\left.\mathrm{CDCl}_{3}\right) \delta 30.0 ; \mathrm{FTIR}\left(\mathrm{cm}^{-1}\right): 2978,1621,1355,1145$. HRMS (Cl) m/z, calcd for $\left[\mathrm{C}_{15} \mathrm{H}_{22} \mathrm{BO}_{2}\right]^{+}:$245.1713; found: 245.1710.

\section{Preparation of Non-Pinacol Containing Boronic Esters:}<smiles>CC1CC(C)(C)OB(/C=C/c2ccc(C(C)(C)C)cc2)O1</smiles>

(30) Using a modification of general procedure $\mathrm{B}$, catecholchloroborane (694 mg, $4.5 \mathrm{mmol}),(\mathrm{L} 1)_{2} \mathrm{PdCl}_{2}$ (83 mg, $0.075 \mathrm{mmol})$, lithium iodide $(20 \mathrm{mg}, 0.15 \mathrm{mmol}), \mathrm{N}, \mathrm{N}-$ dicyclohexylmethylamine $(3.2 \mathrm{~mL}, 5.0 \mathrm{mmol})$, trifluorotoluene $(6.0 \mathrm{~mL}, 0.5 \mathrm{M})$, and 4-tert-butylstyrene $(481 \mathrm{mg}, 3.0 \mathrm{mmol})$ were combined under $\mathrm{N}_{2}$ and stirred at $70{ }^{\circ} \mathrm{C}$ for $24 \mathrm{~h}$. After 24 $\mathrm{h}$, the reaction was removed from the oil bath and opened to air. 2-Methyl-2,4-pentanediol $(1.2 \mathrm{~mL}, 9.0 \mathrm{mmol})$ was added in one portion and the reaction was allowed to cool to rt with stirring. Once at rt, the reaction was diluted with $20 \mathrm{~mL}$ of diethyl ether. The reaction was stirred for $10 \mathrm{~min}$, filtered through Celite and concentrated in vacuo to remove solvents including trifluorotoluene. The crude oil was diluted with diethyl ether $(30 \mathrm{~mL})$ and washed with $1 \mathrm{M}$ hydrochloric acid $(3 \times 20 \mathrm{~mL})$ to remove excess amine. The organic layer was dried with $\mathrm{MgSO}_{4}$, filtered through Celite and concentrated in vacuo. The crude material was purified by boric acid impregnated silica gel chromatography ( $3: 7$ dichloromethane : hexanes) to afford 30 as a slight red solid $(620 \mathrm{mg}, 75 \%):{ }^{1} \mathrm{H}$ NMR $\left(400 \mathrm{MHz}, \mathrm{CDCl}_{3}\right) \delta 7.42(\mathrm{~d}, J=8.3 \mathrm{~Hz}$, 2H), $7.36-7.30(\mathrm{~m}, 3 \mathrm{H}), 6.06(\mathrm{~d}, J=18.2 \mathrm{~Hz}, 1 \mathrm{H}), 4.27$ (ddt, $J=12.0,5.9,3.0 \mathrm{~Hz}, 1 \mathrm{H}), 1.82$ (dd, $J=13.9,3.0 \mathrm{~Hz}, 1 \mathrm{H}), 1.56(\mathrm{t}, J=14.0,11.4 \mathrm{~Hz}, 1 \mathrm{H}), 1.35(\mathrm{~m}, J=2.6 \mathrm{~Hz}, 6 \mathrm{H}), 1.33(\mathrm{~s}, 3 \mathrm{H}), 1.31$ $(\mathrm{s}, 9 \mathrm{H}) ;{ }^{13} \mathrm{C}$ NMR $\left(101 \mathrm{MHz}, \mathrm{CDCl}_{3}\right) \delta 151.5,146.5,135.4,126.8,125.5,71.0,64.9,46.1,34.8$, 31.4, 28.3, 23.4; ${ }^{11} \mathrm{~B}$ NMR $\left(128 \mathrm{MHz}, \mathrm{C}_{6} \mathrm{D} 6\right) \delta 25.6 ; \mathrm{mp}=70-73{ }^{\circ} \mathrm{C} ; \mathrm{FTIR}\left(\mathrm{cm}^{-1}\right): 2970,1623$, 1411, 1391, 1331, 1307, 1267, 1218, 996, 815; HRMS (LIFDI) m/z, calcd for $\left[\mathrm{C}_{18} \mathrm{H}_{27} \mathrm{BO}_{2}\right]^{+}$: 286.2104; found: 286.2111 .<smiles>CC1(C)COB(/C=C/c2ccc(C(C)(C)C)cc2)OC1</smiles>

(31) Using a modification of general procedure $B$, catecholchloroborane $(694 \mathrm{mg}, 4.5 \mathrm{mmol}),(\mathrm{L} 1)_{2} \mathrm{PdCl}_{2}(83 \mathrm{mg}$, $0.075 \mathrm{mmol})$, lithium iodide $(20 \mathrm{mg}, 0.15 \mathrm{mmol}), \mathrm{N}, \mathrm{N}-$ dicyclohexylmethylamine $(3.2 \mathrm{~mL}, 5.0 \mathrm{mmol})$, trifluorotoluene $(6.0 \mathrm{~mL}, 0.5 \mathrm{M})$, and 4-tert-butylstyrene $(481 \mathrm{mg}, 3.0 \mathrm{mmol})$ were combined under $\mathrm{N}_{2}$ and stirred at $70{ }^{\circ} \mathrm{C}$ for $24 \mathrm{~h}$. After 24 $\mathrm{h}$, the reaction was removed from the oil bath and opened to air. 2,2-Dimethyl-1,3-propanediol (937 $\mathrm{mg}, 9.0 \mathrm{mmol}$ ) was added in one portion and the reaction was allowed to cool to rt with stirring. Once at rt, the reaction was diluted with $20 \mathrm{~mL}$ of diethyl ether. The reaction was stirred for $10 \mathrm{~min}$, filtered through Celite and concentrated in vacuo to remove solvents including trifluorotoluene. The crude oil was diluted with diethyl ether $(30 \mathrm{~mL})$ and washed with $1 \mathrm{M}$ hydrochloric acid $(3 \times 20 \mathrm{~mL})$ to remove excess amine. The organic layer was dried with $\mathrm{MgSO}_{4}$, filtered through Celite and concentrated in vacuo. The crude material was purified by boric acid impregnated silica gel chromatography (1:9 ethyl acetate : hexanes) to afford 31 as a white solid $(655 \mathrm{mg}, 80 \%):{ }^{1} \mathrm{H}$ NMR $\left(600 \mathrm{MHz}, \mathrm{CDCl}_{3}\right) \delta 7.43(\mathrm{~d}, \mathrm{~J}=8.3 \mathrm{~Hz}, 2 \mathrm{H})$, $7.35(\mathrm{~d}, J=8.3 \mathrm{~Hz}, 2 \mathrm{H}), 7.31(\mathrm{~d}, J=18.3 \mathrm{~Hz}, 1 \mathrm{H}), 6.06(\mathrm{~d}, J=18.3 \mathrm{~Hz}), 3.70(\mathrm{~s}, 4 \mathrm{H}), 1.31(\mathrm{~s}$, $9 \mathrm{H}), 1.01(\mathrm{~s}, 6 \mathrm{H}) ;{ }^{13} \mathrm{C}$ NMR $\left(151 \mathrm{MHz}, \mathrm{CDCl}_{3}\right) \delta 151.9,147.1,135.2,126.9,125.6,72.4,34.8$, 32.0, 31.4, 22.1; ${ }^{11} \mathrm{~B}$ NMR (193 MHz, CDCl $) \delta 26.3$; FTIR $\left(\mathrm{cm}^{-1}\right): 2962,2872,1622,1513,1476$, $1313,1256,996,815 ; \mathrm{mp}=79-81{ }^{\circ} \mathrm{C}$. HRMS $(\mathrm{Cl}) \mathrm{m} / \mathrm{z}$, calcd for $\left[\mathrm{C}_{17} \mathrm{H}_{26} \mathrm{BO}_{2}\right]^{+}:$273.2026; found: 273.2026 .<smiles>CC(C)(C)c1ccc(/C=C/B2O[C@@H](c3ccccc3)[C@H](c3ccccc3)O2)cc1</smiles>

(32) Using a modification of general procedure $\mathrm{B}$, catecholchloroborane $(694 \mathrm{mg}, 4.5 \mathrm{mmol}),(\mathrm{L} 1)_{2} \mathrm{PdCl}_{2}$ (83 $\mathrm{mg}$, $0.075 \mathrm{mmol})$, lithium iodide $(20 \mathrm{mg}, 0.15 \mathrm{mmol}), \mathrm{N}, \mathrm{N}-$ dicyclohexylmethylamine $(3.2 \mathrm{~mL}, 5.0 \mathrm{mmol})$, trifluorotoluene $(6.0 \mathrm{~mL}, 0.5 \mathrm{M})$, and 4-tert-butylstyrene $(481 \mathrm{mg}, 3.0 \mathrm{mmol})$ were combined under $\mathrm{N}_{2}$ and stirred at $70{ }^{\circ} \mathrm{C}$ for $24 \mathrm{~h}$. After 24 $\mathrm{h}$, the reaction was removed from the oil bath and opened to air. $(1 \mathrm{R}, 2 \mathrm{R})-1,2$-diphenyl-1,2-ethanediol $(1.93 \mathrm{~g}, 9.0 \mathrm{mmol})$ was added in one portion and the 
reaction was allowed to cool to rt with stirring. Once at rt, the reaction was diluted with $20 \mathrm{~mL}$ of diethyl ether. The reaction was stirred for $10 \mathrm{~min}$, filtered through Celite and concentrated in vacuo to remove solvents including trifluorotoluene. The crude oil was diluted with diethyl ether $(30 \mathrm{~mL})$ and washed with $1 \mathrm{M}$ hydrochloric acid $(3 \times 20 \mathrm{~mL})$ to remove excess amine. The organic layer was stirred with ammonium pyrrolidine-dithiocarbamate (palladium scavenger, 6 equiv to palladium $)^{15}$ for $1 \mathrm{~h}$, then dried with $\mathrm{MgSO}_{4}$, filtered through Celite and concentrated in vacuo The crude material was purified by boric acid impregnated silica gel chromatography (1: 4 ethyl acetate : hexanes) to afford 32 as a thick yellow oil $(929 \mathrm{mg}, 81 \%):{ }^{1} \mathrm{H} \mathrm{NMR}\left(600 \mathrm{MHz}, \mathrm{CDCl}_{3}\right) \delta$ $7.61(\mathrm{~d}, J=18.4 \mathrm{~Hz}, 1 \mathrm{H}), 7.50(\mathrm{~d}, J=8.3 \mathrm{~Hz}, 2 \mathrm{H}), 7.43-7.39(\mathrm{~m}, 6 \mathrm{H}), 7.38-7.33(\mathrm{~m}, 6 \mathrm{H}), 6.32$ $(\mathrm{d}, J=18.4 \mathrm{~Hz}, 1 \mathrm{H}), 5.25(\mathrm{~s}, 2 \mathrm{H}), 1.34(\mathrm{~s}, 9 \mathrm{H}) ;{ }^{13} \mathrm{C}$ NMR $\left(101 \mathrm{MHz}, \mathrm{CDCl}_{3}\right) \delta 152.7,151.1,140.4$, 134.7, 129.0, 128.5, 127.2, 126.0, 125.8, 86.7, 34.9, 31.4; ${ }^{11} \mathrm{~B}$ NMR $\left(193 \mathrm{MHz}, \mathrm{CDCl}_{3}\right) \delta 30.8$; FTIR $\left(\mathrm{cm}^{-1}\right): 2963,1622,1344,1319,1175,999,815,761,698$. HRMS (Cl) m/z, calcd for $\left[\mathrm{C}_{26} \mathrm{H}_{28} \mathrm{BO}_{2}\right]^{+}:$383.2182; found: 383.2178.<smiles>CC(C)(C)c1ccc(/C=C/B2Nc3cccc4cccc(c34)N2)cc1</smiles>

(33) Using a modification of general procedure $\mathrm{B}$, catecholchloroborane $(694 \mathrm{mg}, 4.5 \mathrm{mmol}),(\mathrm{L} 1)_{2} \mathrm{PdCl}_{2}(83 \mathrm{mg}$, $0.075 \mathrm{mmol})$, lithium iodide $(20 \mathrm{mg}, 0.15 \mathrm{mmol}), \mathrm{N}, \mathrm{N}-$ dicyclohexylmethylamine $(3.2 \mathrm{~mL}, 5.0 \mathrm{mmol})$, trifluorotoluene $(6.0 \mathrm{~mL}, 0.5 \mathrm{M})$, and 4-tert-butylstyrene $(481 \mathrm{mg}, 3.0 \mathrm{mmol})$ were combined under $\mathrm{N}_{2}$ and stirred at $70{ }^{\circ} \mathrm{C}$ for $24 \mathrm{~h}$. After 24 $\mathrm{h}$, the reaction was removed from the oil bath and opened to air. 1,8-Diaminonapthalene $(1.42 \mathrm{~g}, 9.0 \mathrm{mmol})$ was added in one portion and the reaction was allowed to cool to rt with stirring. Once at $\mathrm{rt}$, the reaction was diluted with $20 \mathrm{~mL}$ of diethyl ether. The reaction was stirred for $10 \mathrm{~min}$, filtered through Celite and concentrated in vacuo to remove solvents including trifluorotoluene. The crude material was purified by boric acid impregnated silica gel chromatography ( $3: 7$ dichloromethane : hexanes) to afford 33 as a yellow solid (664 mg, 85\%): ${ }^{1} \mathrm{H}$ NMR $\left(400 \mathrm{MHz}, \mathrm{CDCl}_{3}\right) \delta 7.46(\mathrm{~d}, J=8.4 \mathrm{~Hz}, 2 \mathrm{H})$, $7.41(\mathrm{~d}, J=8.4 \mathrm{~Hz}, 2 \mathrm{H}), 7.15(\mathrm{~d}, J=10.5 \mathrm{~Hz}, 1 \mathrm{H}), 7.11(\mathrm{~d}, J=7.5 \mathrm{~Hz}, 2 \mathrm{H}), 7.02(\mathrm{~d}, J=8.1 \mathrm{~Hz}$, 2H), $6.37(\mathrm{~d}, J=7.3 \mathrm{~Hz}, 2 \mathrm{H}), 6.29(\mathrm{~d}, J=18.6 \mathrm{~Hz}, 1 \mathrm{H}), 5.86(\mathrm{~s}, 2 \mathrm{H}), 1.34(\mathrm{~s}, 9 \mathrm{H}) ;{ }^{13} \mathrm{C}$ NMR $(101$ $\left.\mathrm{MHz}, \mathrm{CDCl}_{3}\right) \delta 152.1,143.7,141.3,136.5,134.9,127.7,126.7,125.8,120.0,117.7,105.9,34.9$, 31.4; ${ }^{11} \mathrm{~B}$ NMR $\left(193 \mathrm{MHz}, \mathrm{CDCl}_{3}\right) \delta 28.9 ; \mathrm{FTIR}\left(\mathrm{cm}^{-1}\right): 2962,1600,1513,1413,818,762 ; \mathrm{mp}=$ 135-136 ${ }^{\circ} \mathrm{C}$. HRMS (ESI) m/z, calcd for $\left[\mathrm{C}_{22} \mathrm{H}_{24} \mathrm{BN}_{2}\right]^{+}$: 327.2027 ; found: 327.2041 .<smiles></smiles>

(34) Using a modification of general procedure $\mathrm{B}$, catecholchloroborane $(694 \mathrm{mg}, 4.5 \mathrm{mmol}),(\text { L1 })_{2} \mathrm{PdCl}_{2}(83 \mathrm{mg}, 0.075$ $\mathrm{mmol})$, lithium iodide $(20 \mathrm{mg}, \quad 0.15 \mathrm{mmol}), \mathrm{N}, \mathrm{N}-$ dicyclohexylmethylamine $(3.2 \mathrm{~mL}, 5.0 \mathrm{mmol})$, trifluorotoluene $(6.0 \mathrm{~mL}$, $0.5 \mathrm{M}$ ), and 4-tert-butylstyrene $\left(481 \mathrm{mg}, 3.0 \mathrm{mmol}\right.$ ) were combined under $\mathrm{N}_{2}$ and stirred at $70{ }^{\circ} \mathrm{C}$ for $24 \mathrm{~h}$. After $24 \mathrm{~h}$, the reaction was removed from the oil bath and allowed to cool to rt with stirring. Once at rt, the reaction was diluted with $20 \mathrm{~mL}$ of diethyl ether. The reaction was stirred for $10 \mathrm{~min}$, filtered through Celite and concentrated in vacuo to remove solvents including trifluorotoluene. The crude oil was diluted with diethyl ether $(30 \mathrm{~mL})$ and washed with $1 \mathrm{M}$ hydrochloric acid $(3 \times 20 \mathrm{~mL})$ to remove excess amine. The organic layer was dried with $\mathrm{MgSO}_{4}$, filtered through Celite and concentrated in vacuo. The crude solid was diluted with diethyl ether $(10 \mathrm{~mL})$ and cooled $0{ }^{\circ} \mathrm{C}$ in an ice bath. Once at $0{ }^{\circ} \mathrm{C}$, potassium bifluoride $(1.41 \mathrm{~g}, 18.0 \mathrm{mmol})$ was added with stirring. Deionized water $(3 \mathrm{~mL})$ was then added dropwise over 30 minutes, at which time the ice bath was removed and the reaction was allowed to warm to $\mathrm{rt}$ with stirring. Once at $\mathrm{rt}$, the solvent was removed in vacuo. The crude solid was dissolved in acetone $(20 \mathrm{ml})$, filtered through Celite and concentrated in vacuo. The crude material was purified by dissolving in hot acetone $(10 \mathrm{~mL})$ and precipitating with diethyl ether $(20 \mathrm{~mL})$ to afford 34 as a white solid (564 $\mathrm{mg}, 70 \%):{ }^{1} \mathrm{H}$ NMR $(600 \mathrm{MHz}$, Acetone-d6) $\delta 7.27(\mathrm{~s}, 4 \mathrm{H}), 6.62(\mathrm{~d}, \mathrm{~J}=18.2 \mathrm{~Hz}, 1 \mathrm{H}), 6.29(\mathrm{~d}, \mathrm{~J}=$ $18.0 \mathrm{~Hz}, 1 \mathrm{H}), 1.28(\mathrm{~s}, 9 \mathrm{H}) ;{ }^{13} \mathrm{C}$ NMR $(101 \mathrm{MHz}$, Acetone-d6) $\delta 149.1,139.2,134.3$ (q), 126.2, 125.7, 34.8, 31.7; ${ }^{11} \mathrm{~B}$ NMR (193 MHz, Acetone-d6) $\delta 3.0 ;{ }^{19} \mathrm{~F}$ NMR (376 MHz, Acetone-d6) $\delta-$ 141.6; FTIR $\left(\mathrm{cm}^{-1}\right): 3647,2966,2869,1705,1627,1563,1464,1412,1393,1364,1296,1098$, $966,855,754,560,514 ; \mathrm{mp}=263-267^{\circ} \mathrm{C}$ (dec.). HRMS (LIFDI) $\mathrm{m} / \mathrm{z}$, calcd for $\left[\mathrm{C}_{12} \mathrm{H}_{15} \mathrm{BF}_{3} \mathrm{~K}\right]$ : 266.0856; found: 266.0872 . 


\section{Additional Optimization Data:}

Note: All reactions in this section were performed on $0.25 \mathrm{mmol}$ in a nitrogen-filled glovebox.

\section{Examination of Palladium Sources:}

During initial optimization it was found that many palladium precatalysts are competent in this reaction (Table S1). Using otherwise optimized reaction conditions, a more extensive survey of palladium precatalysts was conducted (Table S2). The results from those investigations are shown below.

Table S1. Examination of Alternate Palladium Sources with $\mathrm{Cy}_{3} \mathrm{P}$.

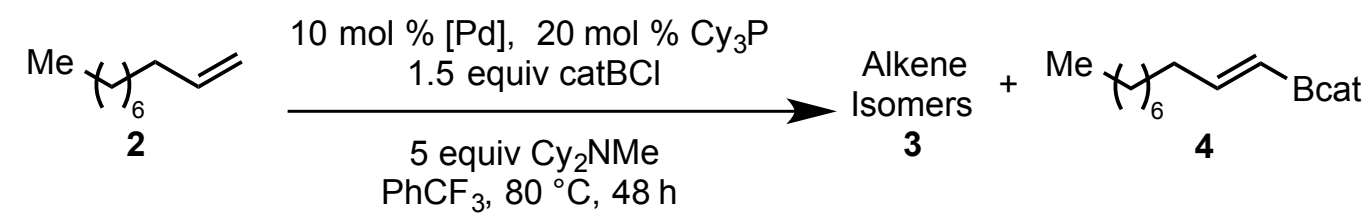

$\begin{array}{ccccc}\text { Entry } & {[\mathrm{Pd}] \mathrm{Source}} & \mathbf{\% \mathbf { 2 } ^ { \mathrm { a } }} & \mathbf{\% 3}^{\mathrm{a}} & \mathbf{\% 4}^{\mathrm{a}} \\ 1 & (\mathrm{COD}) \mathrm{Pd}\left(\mathrm{CH}_{2} \mathrm{SiMe}_{3}\right)_{2} & 0 & 38 & 62 \\ 2 & \mathrm{Pd}_{2} \mathrm{dba}_{3} & 0 & 44 & 56 \\ 3 & \mathrm{Pd}(\mathrm{dba})_{2} & 0 & 37 & 63 \\ 4 & {[(\mathrm{allyl}) \mathrm{PdCl}]_{2}} & 0 & 38 & 62\end{array}$

${ }^{a}$ Yields obtained by ${ }^{1} \mathrm{H}$ NMR.

Table S2. Examination of Alternate Palladium Sources with L1.

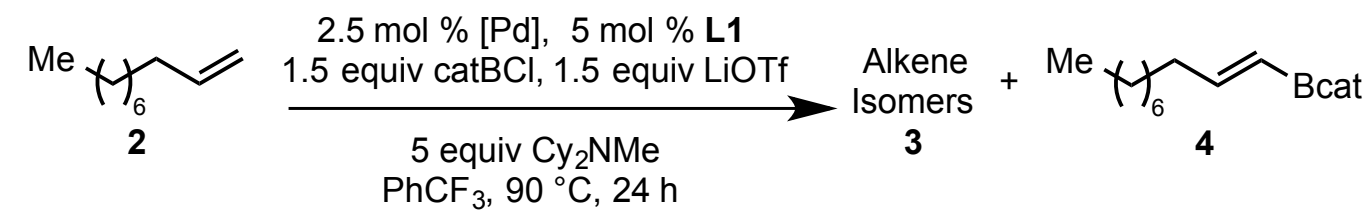

$\begin{array}{ccccc}\text { Entry } & {[\mathrm{Pd}] \mathrm{Source}} & \% \mathbf{2}^{a} & \% \mathbf{3}^{a} & \% \mathbf{4}^{a} \\ 1 & \mathrm{Pd}_{2} \mathrm{dba}_{3} & 0 & 0 & >99 \\ 2 & \mathrm{Pd}_{2} \mathrm{dba}_{3} \cdot \mathrm{CHCl}_{3} & 0 & 0 & >99 \\ 3 & \mathrm{Pd}(\mathrm{dba})_{2} & 0 & 0 & >99 \\ 4 & \mathrm{PdCl}_{2} & 0 & 12 & 88 \\ 5 & (\mathrm{MeCN})_{2} \mathrm{PdCl}_{2} & 0 & 13 & 87 \\ 6 & (\mathrm{cod}) \mathrm{PdCl}_{2} & 0 & 0 & >99 \\ 7 & {\left[(\mathrm{cinnamyl}) \mathrm{PdCl}_{2}\right.} & 0 & 0 & >99 \\ 8 & \mathrm{Pdd}]_{2} & 0 & 36 & 64 \\ 9 & \mathrm{Pd}(\mathrm{OAc})_{2} & 0 & 78 & 22 \\ 10 & (\mathrm{MeCN})_{4} \mathrm{Pd}_{2}\left(\mathrm{BF}_{4}\right)_{2} & 0 & 1 & 99 \\ 11 & (\mathrm{L1})_{2} \mathrm{Pd}^{b} & 0 & 0 & >99 \\ 12 & \mathrm{none} & >99 & 0 & 0\end{array}$

${ }^{a}$ Yields obtained by ${ }^{1} \mathrm{H}$ NMR with ferrocene as an internal standard. ${ }^{b}$ No external ligand added. 
Supporting Information for Reid et al.

\section{Examination of Ligands with $\mathrm{Pd}_{2} \mathrm{dba}_{3}$ :}

During reaction optimization, additional ligands were examined than were discussed in the main text. The full table of results is shown below (Table S3).

Table S3. Full Table of Ligands.

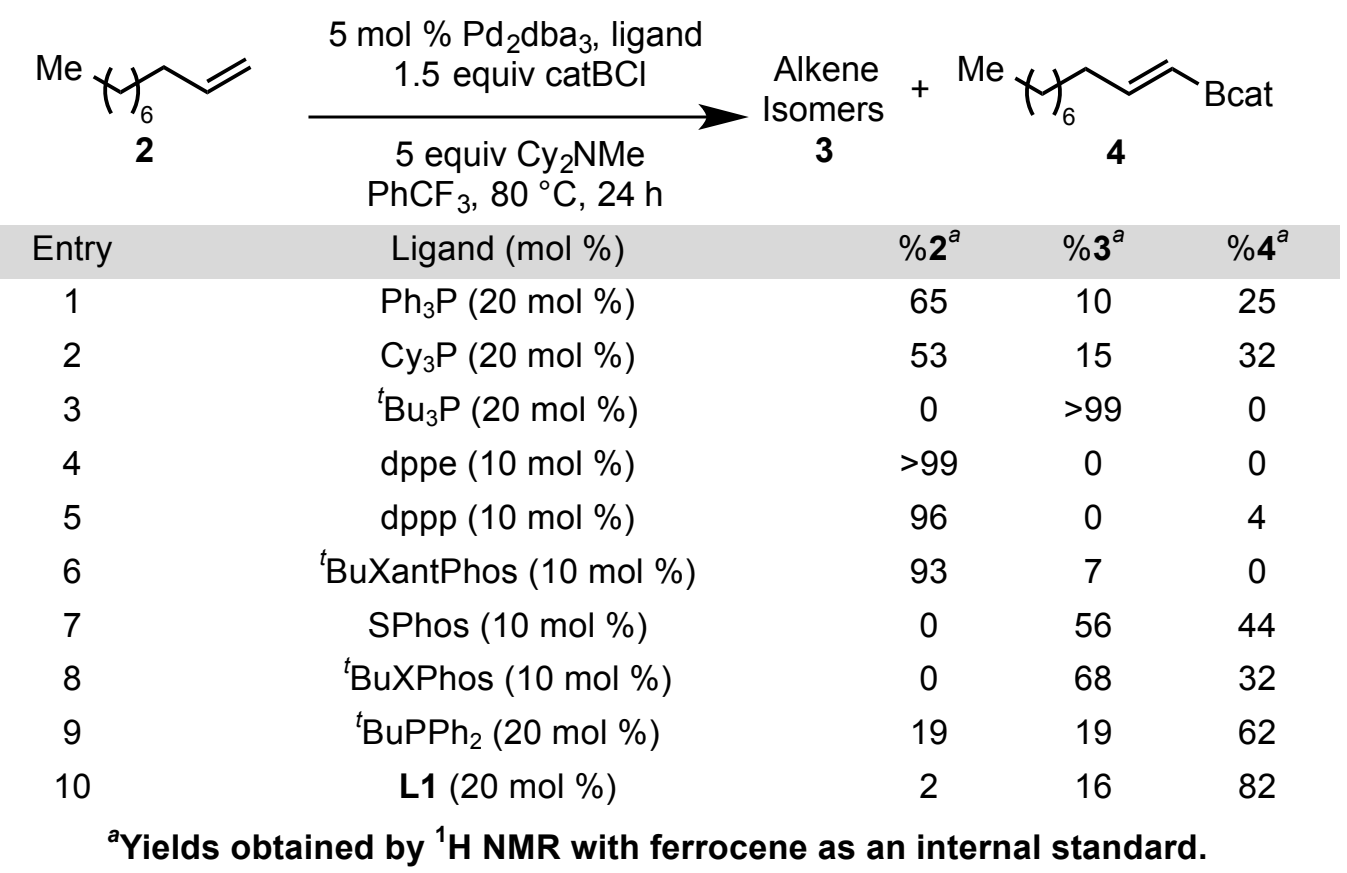




\section{Computational and Experimental Mechanistic Insight:}

\section{Computational Methods:}

All DFT calculations were performed with the Gaussian 09 software package. ${ }^{16}$ Optimizations of the geometries of the minima were conducted with the B3LYP ${ }^{17-19}$ method. The $6-31++G(d, p)$ basis set was applied to all atoms. Vibrational frequencies were computed at the same level of theory for each calculation to verify that the structure is an energy minimum and to evaluate the thermochemical properties. Solvent effects were computed based upon the gas-phase optimized structures using the same level of theory with the default polarized continuum solvation model (PCM) in Gaussian. ${ }^{20}$ 1,2-Dichloroethane $(\varepsilon=10.13)$ was used instead of trifluorotoluene $(\varepsilon=$ 9.18) because the Gaussian 09 program does not contain default solvent parameters for trifluorotoluene.$^{21}$ In this communication, all the energies are discussed in terms of enthalpy.

\section{Computational Coordination Studies:}

Density functional theory calculations (B3LYP/6-31++G(d,p)) modeling this coordination also suggest that $\mathrm{Cy}_{2} \mathrm{NMe}$ coordinates more reversibly with catBCl (Figure S2). Examination of the $\Delta \mathrm{H}$ of the coordination complexes compared to the free amine and catBCl show $-7.2 \mathrm{kcal} / \mathrm{mol}$ with triethylamine but only $-3.2 \mathrm{kcal} / \mathrm{mol}$ with $\mathrm{Cy}_{2} \mathrm{NMe}$. The stability of catBCl$\cdot \mathrm{Et}_{3} \mathrm{~N}$ sequesters the cat $\mathrm{BCl}$, preventing it from entering the catalytic cycle.

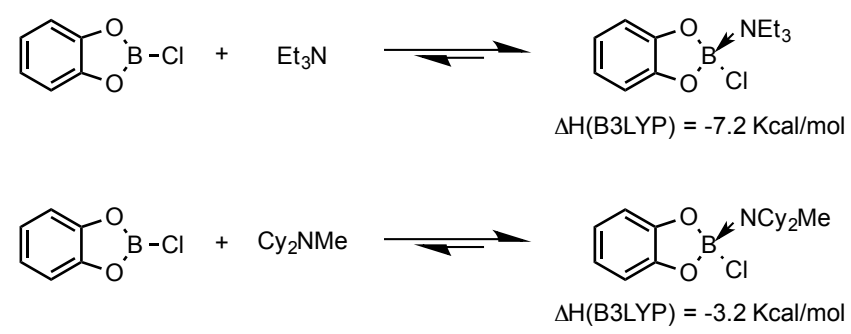

Figure S2. DFT calculations of coordination complexes.

\section{NMR Coordination Studies:}

Although the combination of catBCl and $\mathrm{Cy}_{2} \mathrm{NMe}$ (in $\mathrm{CDCl}_{3}$ ) does reveal formation of an amineborane adduct by ${ }^{11} \mathrm{~B}$ NMR (Figure S3, S7), it is distinct from that formed with $\mathrm{Et}_{3} \mathrm{~N}$ (Figure S3, A). The $\mathrm{Cy}_{2} \mathrm{NMe}$ derived complex is broader and more downfield (Figure S3, B), both of which indicate a less tightly bound boron atom. Further, addition of 1 equiv of $\mathrm{Et}_{3} \mathrm{~N}$ to $\mathbf{S 7}$ results in complete conversion to 1 (Figure S3, C), providing additional evidence for a less stable $\mathrm{Cy}_{2} \mathrm{NMe}$ complex. This data supports the notion that a greater concentration of free catBCl is available with the use of the larger base and explains our success when compared to earlier reports.

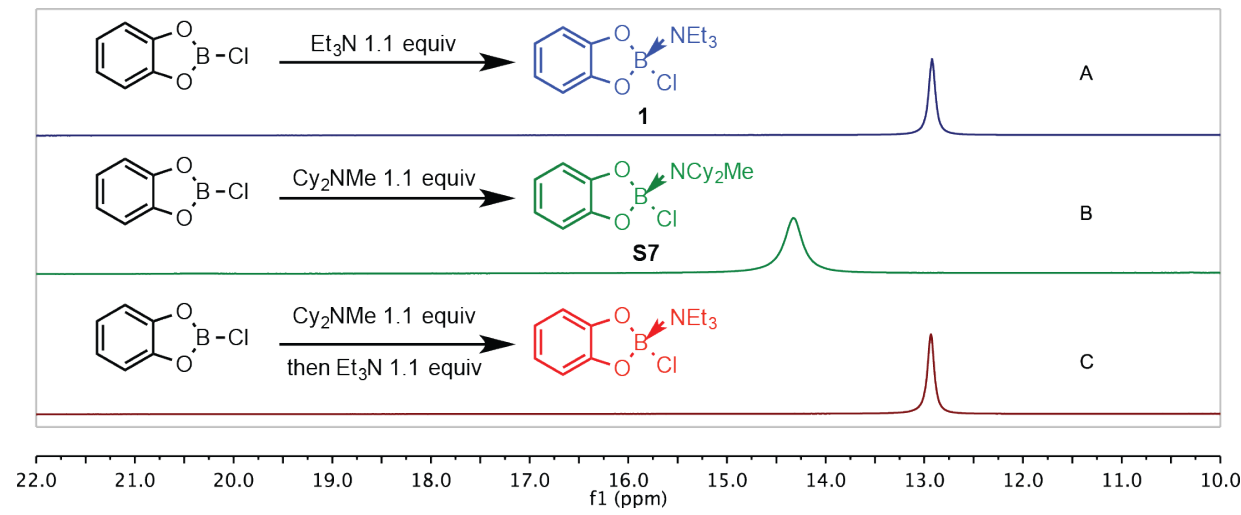

Figure S3. ${ }^{11} \mathrm{~B}$ NMR spectra of coordination complexes. 


\section{Computational Investigation of $E / Z$ Selectivity:}

We sought to compare some of the experimental $E / Z$ ratios to calculated DFT energies for several substrates. All of the following calculations (B3LYP/6-31++G(d,p)) were performed at 90 ${ }^{\circ} \mathrm{C}$. Ground state calculations for each of the four possible butene product isomers were performed to determine the relative $\Delta \mathrm{H}$. The products of 1-butene were chosen because they should be representative of all larger a-olefines. The computed and experimentally measured $E$ alkenyl, Z-alkenyl, E-allyl and Z-allyl products correlate very well supporting the notion that the observed ratio is thermodynamic (Table S4). The products from allylbenzene and styrene were also examined and show similar correlations (Tables S5 and S6).

Table S4. Calculated and Experimental Values for $E / Z$ ratios of Butene Products

\begin{tabular}{|c|c|c|c|c|}
\hline entry & structure & $\begin{array}{c}\text { relative energy } \\
\boldsymbol{\Delta H} \text { B3LYP }\end{array}$ & $\begin{array}{c}\text { theoretical } \\
\text { ratio }\end{array}$ & $\begin{array}{c}\text { experimental ratio } \\
\text { for compound 5 }\end{array}$ \\
\hline 1 & $\mathrm{Me}_{\mathrm{Bcat}}$ & $0.0 \mathrm{kcal} / \mathrm{mol}$ & 92 & 89 \\
\hline 2 & $\mathrm{Me} \widetilde{\mathrm{Bcat}}$ & $1.9 \mathrm{kcal} / \mathrm{mol}$ & 6 & 11 \\
\hline 3 & $\mathrm{Me} \sim_{\mathrm{Bcat}}$ & $2.8 \mathrm{kcal} / \mathrm{mol}$ & 2 & 0 \\
\hline 4 & $\mathrm{Me}^{\prime-\sim_{\mathrm{Bcat}}}$ & $4.3 \mathrm{kcal} / \mathrm{mol}$ & 0 & 0 \\
\hline
\end{tabular}

Table S5. Calculated and Experimental Values for E/Z ratios of Styrene Products

\begin{tabular}{|c|c|c|c|c|}
\hline entry & structure & $\begin{array}{c}\text { relative energy } \\
\Delta \mathrm{H} \mathrm{B3LYP}\end{array}$ & $\begin{array}{c}\text { theoretical } \\
\text { ratio }\end{array}$ & $\begin{array}{c}\text { experimental ratio } \\
\text { for compound 19 }\end{array}$ \\
\hline 1 & $0.0 \mathrm{kcal} / \mathrm{mol}$ & 100 & 100 \\
\hline 2 & $4.9 \mathrm{kcal} / \mathrm{mol}$ & 0 & 0 \\
\hline
\end{tabular}

Table S6. Calculated and Experimental Values for E/Z ratios of Allyl Benzene Products

\begin{tabular}{|c|c|c|c|c|}
\hline entry & structure & $\begin{array}{c}\text { relative energy } \\
\Delta \mathrm{H} \text { B3LYP }\end{array}$ & $\begin{array}{l}\text { theoretical } \\
\text { ratio }\end{array}$ & $\begin{array}{l}\text { experimental ratio } \\
\text { for compound } 18\end{array}$ \\
\hline 1 & Bcat & $0.0 \mathrm{kcal} / \mathrm{mol}$ & 56 & 67 \\
\hline 2 & Bcat & $2.9 \mathrm{kcal} / \mathrm{mol}$ & 1 & 5 \\
\hline 3 & Bcat & $0.2 \mathrm{kcal} / \mathrm{mol}$ & 40 & 25 \\
\hline 4 & $\overline{7}_{\text {Bcat }}$ & $2.1 \mathrm{kcal} / \mathrm{mol}$ & 3 & 3 \\
\hline
\end{tabular}




\section{Isomerization Study:}

The reference NMR spectra used to determine the isomerization of each alkene (Figure S4, A-C) and the crude NMR spectrum (D) are shown below. (E)-1-hexenyl-catecholborane was prepared according to a literature procedure. ${ }^{22}$ The control experiment, without catalyst, shows no isomerization of $(E)$-1-hexenyl-catecholborane suggesting that the isomerization is palladium catalyzed.

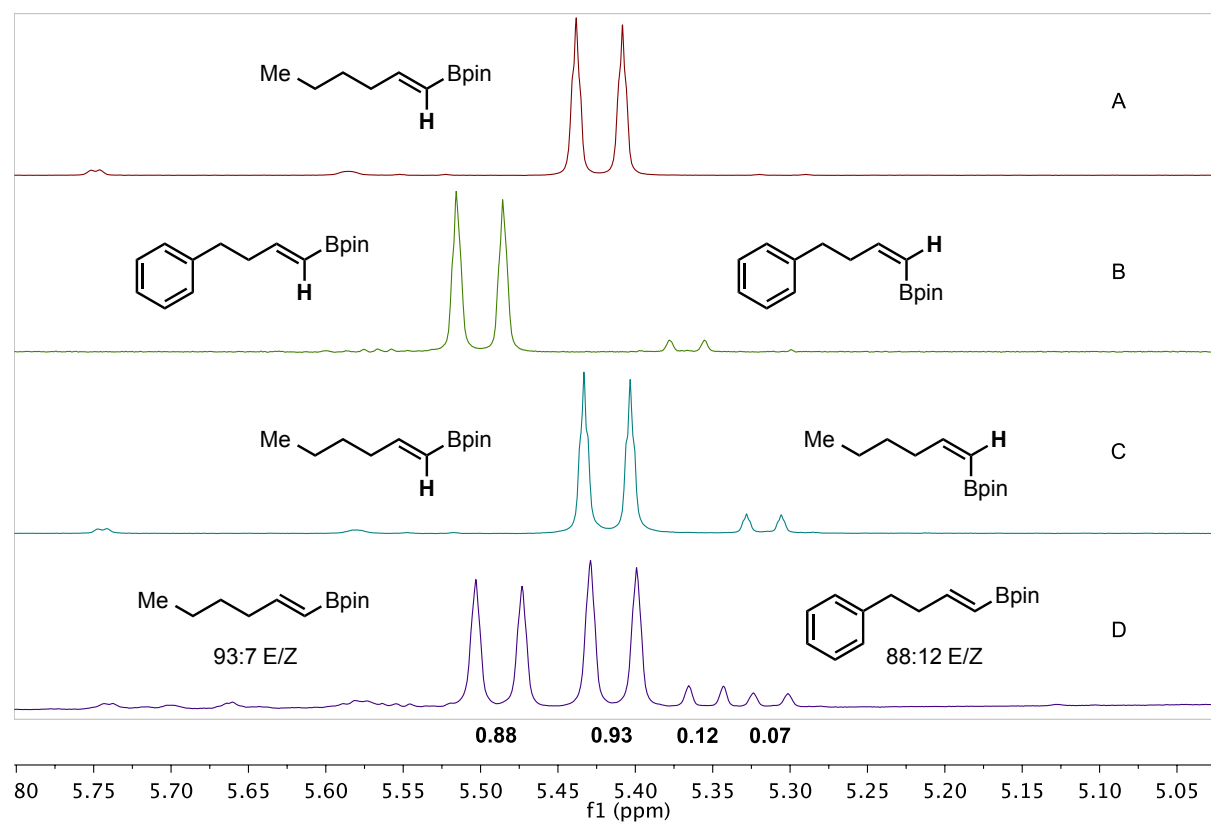

Figure S4. (A) ${ }^{1} \mathrm{H}$ NMR spectrum of $(E)$-1-hexenyl-pinacolborane, $(B){ }^{1} \mathrm{H}$ NMR spectrum of $E$ and $Z$ isomers of 4-phenylbutene products, (C) ${ }^{1} \mathrm{H}$ NMR spectrum of $E$ and $Z$ isomers of 1 -hexene products, (D) ${ }^{1} \mathrm{H}$ NMR spectrum of crude reaction mixture (Equation 2 ).

\section{Isomerization Study Procedure:}

In a nitrogen-filled glovebox, to a 1 dram vial with a stir bar, was added $(\mathbf{L} 1)_{2} \mathrm{PdCl}_{2}(7.0 \mathrm{mg}$, $0.0063 \mathrm{mmol}, 2.5 \mathrm{~mol} \%$ ), catecholchloroborane (58 mg, $0.375 \mathrm{mmol}, 1.5$ equiv), and lithium trifluoromethanesulfonate ( $58 \mathrm{mg}, 0.375 \mathrm{mmol}, 1.5$ equiv) followed by $500 \mu \mathrm{L}$ of trifluorotoluene. $\mathrm{N}, \mathrm{N}$-dicyclohexylmethylamine $(267 \mu \mathrm{L}, 1.25 \mathrm{mmol}, 5.0$ equiv) was added and the reaction was capped and stirred at $90{ }^{\circ} \mathrm{C}$. After $10 \mathrm{~min}, 4$-phenylbutene $(38 \mathrm{mg}, 0.25 \mathrm{mmol}, 1.0$ equiv) and $(E)$ 1-hexenyl-catecholborane ( $50 \mathrm{mg}, 0.25 \mathrm{mmol}, 1.0$ equiv) were added via micropipette and the reaction was sealed and stirred at $90{ }^{\circ} \mathrm{C}$ for $24 \mathrm{~h}$. The reaction was quenched with pinacol (176 $\mathrm{mg}, 1.5 \mathrm{mmol}, 6.0$ equiv) and stirred for $1 \mathrm{~h}$ at rt. The crude mixture was diluted with $1 \mathrm{~mL}$ of diethyl ether, filtered through Celite and concentrated in vacuo. The $E / Z$ ratio was determined via ${ }^{1} \mathrm{H}$ NMR of the crude reaction mixture in $\mathrm{CDCl}_{3}$ (Figure S4, D). 


\section{Crystallographic Details:}

X-ray Structural Analysis for $(\mathrm{L} 1)_{2} \mathrm{PdCl}_{2}$. The crystal was mounted using viscous oil onto a plastic mesh and cooled to the data collection temperature $(200 \mathrm{~K})$. Data were collected on a Bruker-AXS APEX Duo CCD diffractometer. Unit cell parameters were obtained from 36 data frames, $0.3^{\circ} \mathrm{\omega}$, from three different sections of the Ewald sphere. No symmetry higher than triclinic was observed and the centrosymmetric space group option yielded chemically reasonable and computationally stable results of refinement. The data-set was treated with absorption corrections based on redundant multiscan data. The structure was solved using direct methods and refined with full-matrix, least-squares procedures on $F^{2}{ }^{23}$ The molecule is located at the inversion center.

All non-hydrogen atoms were refined with anisotropic displacement parameters. All hydrogen atoms were treated as idealized contributions. Scattering factors are contained in the SHELXTL 6.12 program library. ${ }^{23}$ The CIF has been deposited under CCDC 1463772. 


\section{References:}

(1) Pangborn, A. B.; Giardello, M. A.; Grubbs, R. H.; Rosen, R. K.; Timmers, F. J. Organometallics 1996, 15, 1518.

(2) McAtee, J. R.; Martin, S. E. S.; Ahneman, D. T.; Johnson, K. A.; Watson, D. A. Angew. Chem. Int. Ed. 2012, 51, 3663.

(3) McAtee, J. R.; Yap, G. P. A.; Watson, D. A. J. Am. Chem. Soc. 2014, 136, 10166.

(4) Martin, S. E. S.; Watson, D. A. J. Am. Chem. Soc. 2013, 135, 13330.

(5) Aslam, S. N.; Stevenson, P. C.; Phythian, S. J.; Veitch, N. C.; Hall, D. R. Tetrahedron 2006, 62, 4214.

(6) Waser, J.; Gaspar, B.; Nambu, H.; Carreira, E. M. J. Am. Chem. Soc. 2006, 128, 11693.

(7) Wrackmeyer, B. Prog. Nucl. Magn. Reson. Spectrosc. 1979, 12, 227.

(8) Doi, T.; Fukuyama, T.; Minamino, S.; Husson, G.; Ryu, I. Chem. Commun. 2006, 1875.

(9) Peñafiel, I.; Pastor, I. M.; Yus, M. Tetrahedron 2010, 66, 2928.

(10) Nakamura, M.; Hara, K.; Hatakeyama, T.; Nakamura, E. Org. Lett. 2001, 3, 3137.

(11) Itoh, T.; Matsueda, T.; Shimizu, Y.; Kanai, M. Chem. Eur. J. 2015, 21, 15955.

(12) He, X.; Hartwig, J. F. Organometallics 1996, 15, 400.

(13) Bettinger, H. F.; Filthaus, M.; Bornemann, H.; Oppel, I. M. Angew. Chem. Int. Ed. 2008, 47, 4744.

(14) Hitosugi, S.; Tanimoto, D.; Nakanishi, W.; Isobe, H. Chem. Lett. 2012, 41, 972.

(15) Gallagher, W. P.; Vo, A. Org. Process Res. Dev. 2015, 19, 1369.

(16) Frisch, M. J.; Trucks, G. W.; Schlegel, H. B.; Scuseria, G. E.; Robb, M. A.; Cheeseman, J. R.; Scalmani, G.; Barone, V.; Mennucci, B.; Petersson, G. A.; Nakatsuji, H.; Caricato, M.; Li, X.; Hratchian, H. P.; Izmaylov, A. F.; Bloino, J.; Zheng, G.; Sonnenberg, J. L.; Hada, M.; Ehara, M.; Toyota, K.; Fukuda, R.; Hasegawa, J.; Ishida, M.; Nakajima, T.; Honda, Y.; Kitao, O.; Nakai, H.; Vreven, T.; Montgomery Jr., J. A.; Peralta, J. E.; Ogliaro, F.; Bearpark, M. J.; Heyd, J.; Brothers, E. N.; Kudin, K. N.; Staroverov, V. N.; Kobayashi, R.; Normand, J.; Raghavachari, K.; Rendell, A. P.; Burant, J. C.; lyengar, S. S.; Tomasi, J.; Cossi, M.; Rega, N.; Millam, N. J.; Klene, M.; Knox, J. E.; Cross, J. B.; Bakken, V.; Adamo, C.; Jaramillo, J.; Gomperts, R.; Stratmann, R. E.; Yazyev, O.; Austin, A. J.; Cammi, R.; Pomelli, C.; Ochterski, J. W.; Martin, R. L.; Morokuma, K.; Zakrzewski, V. G.; Voth, G. A.; Salvador, P.; Dannenberg, J. J.; Dapprich, S.; Daniels, A. D.; Farkas, Ö.; Foresman, J. B.; Ortiz, J. V.; Cioslowski, J.; Fox, D. J.; Gaussian, Inc.: Wallingford, CT, USA, 2009.

(17) Becke, A. D. Phys. Rev. A 1988, 38, 3098.

(18) Lee, C.; Yang, W.; Parr, R. G. Phys. Rev. B 1988, 37, 785.

(19) Becke, A. D. J. Chem. Phys. 1993, 98, 5648.

(20) Cossi, M.; Rega, N.; Scalmani, G.; Barone, V. J. Comput. Chem. 2003, 24, 669.

(21) Belding, L.; Chemler, S. R.; Dudding, T. J. Org. Chem. 2013, 78, 10288.

(22) Fairlamb, I. J. S.; Marrison, L. R.; Dickinson, J. M.; Lu, F.-J.; Schmidt, J. P. Bioorg. Med. Chem. 2004, 12, 4285.

(23) Sheldrick, G. Acta Cryst. A 2008, 64, 112. 


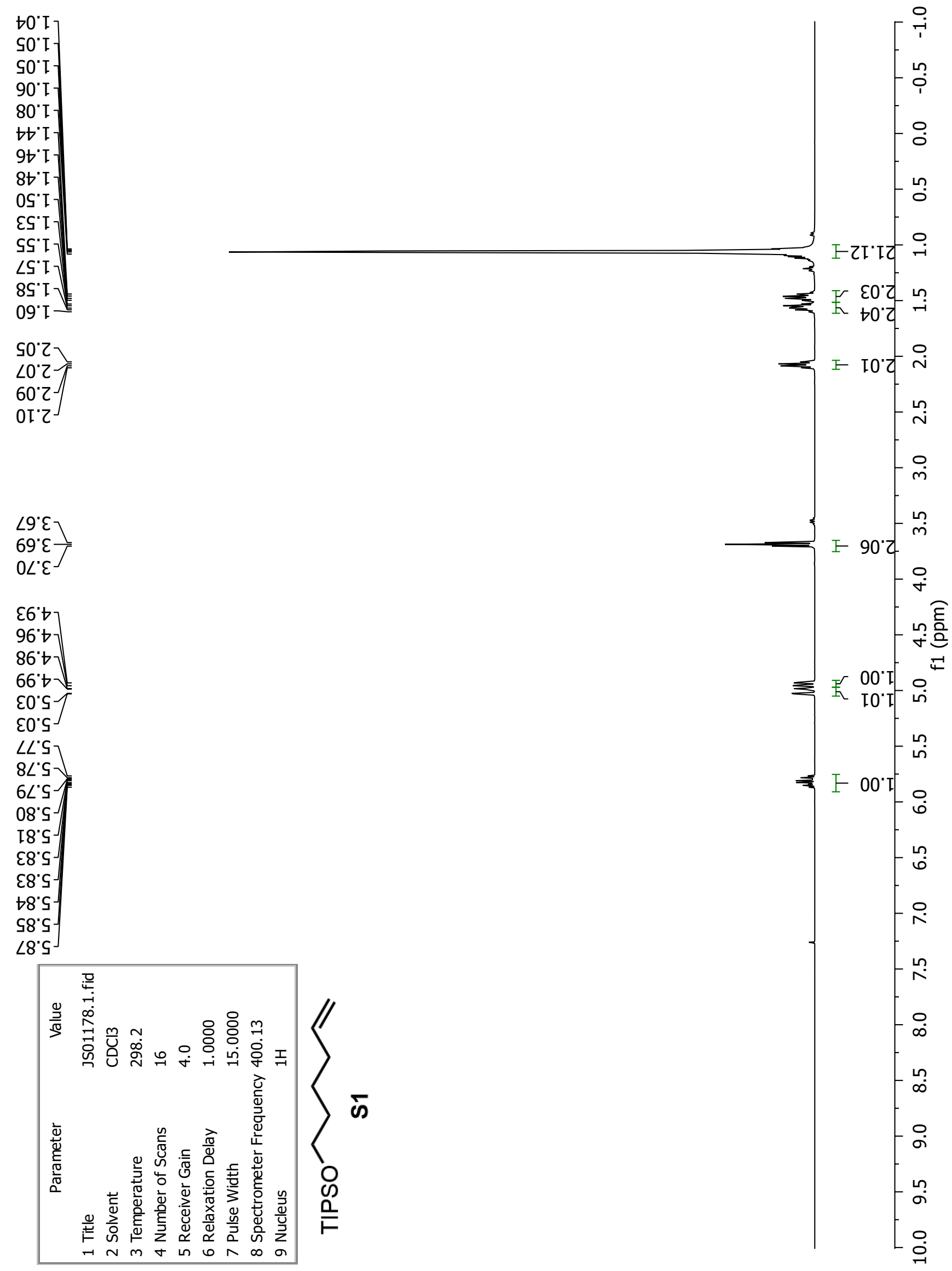



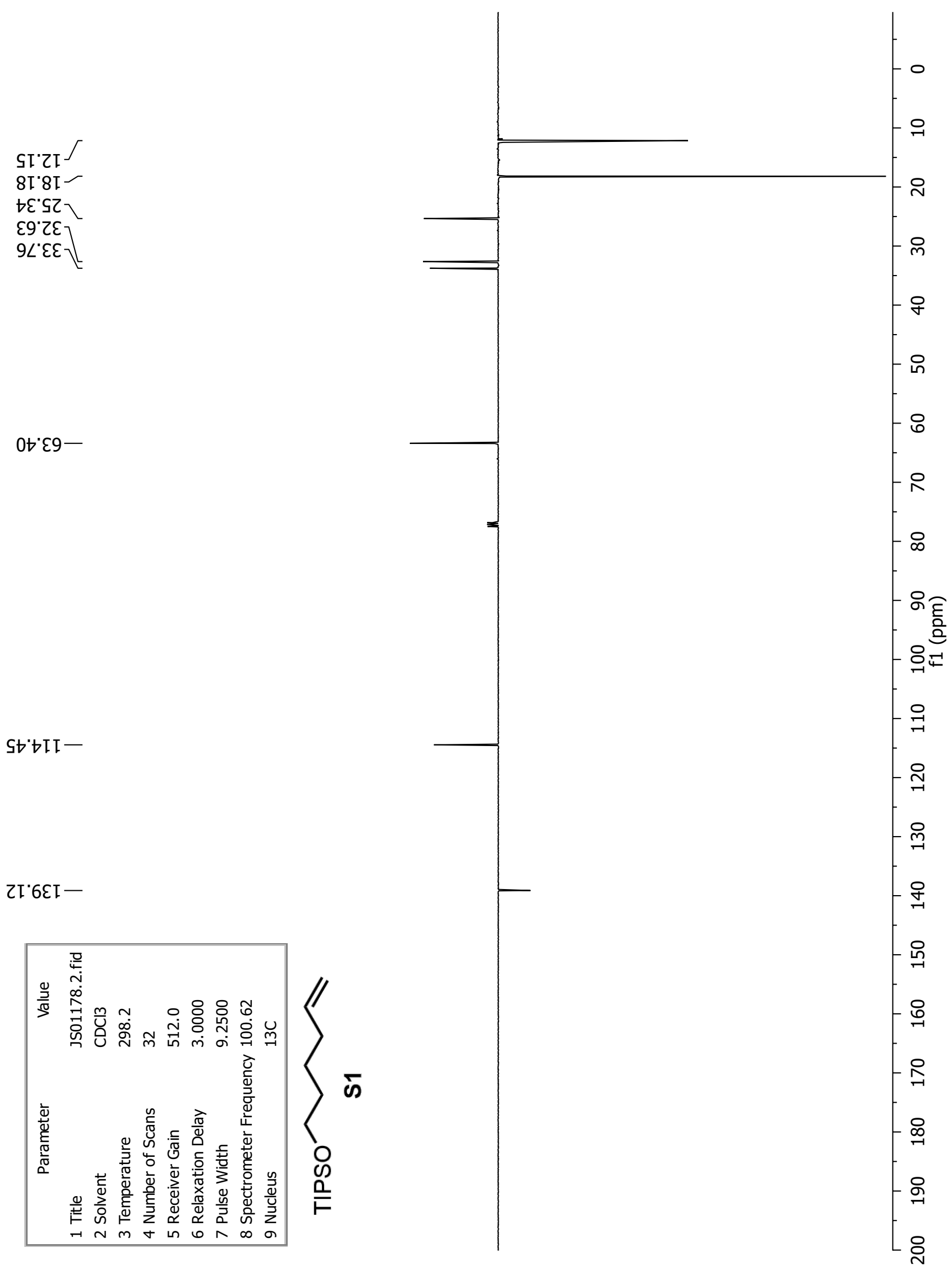

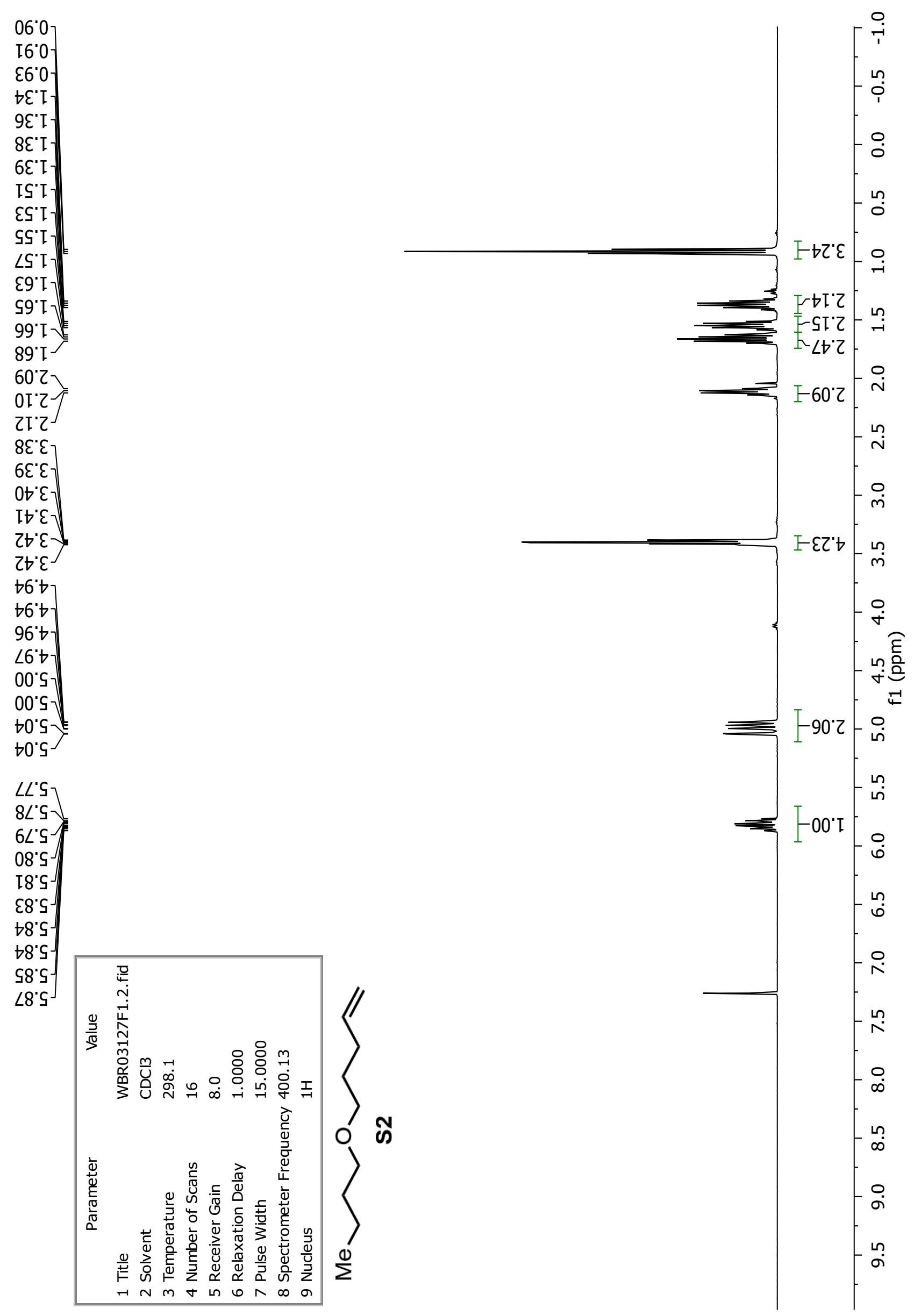


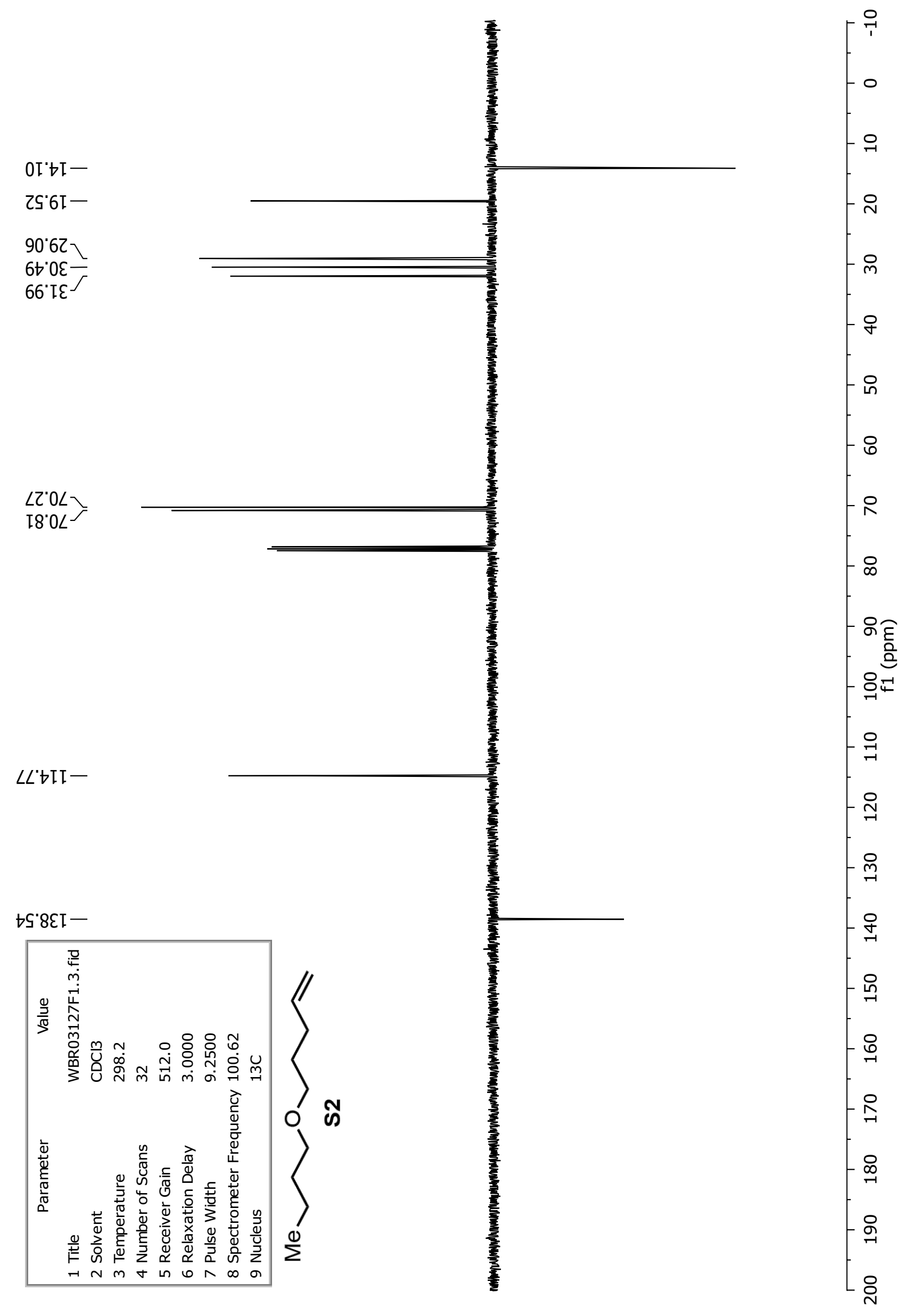




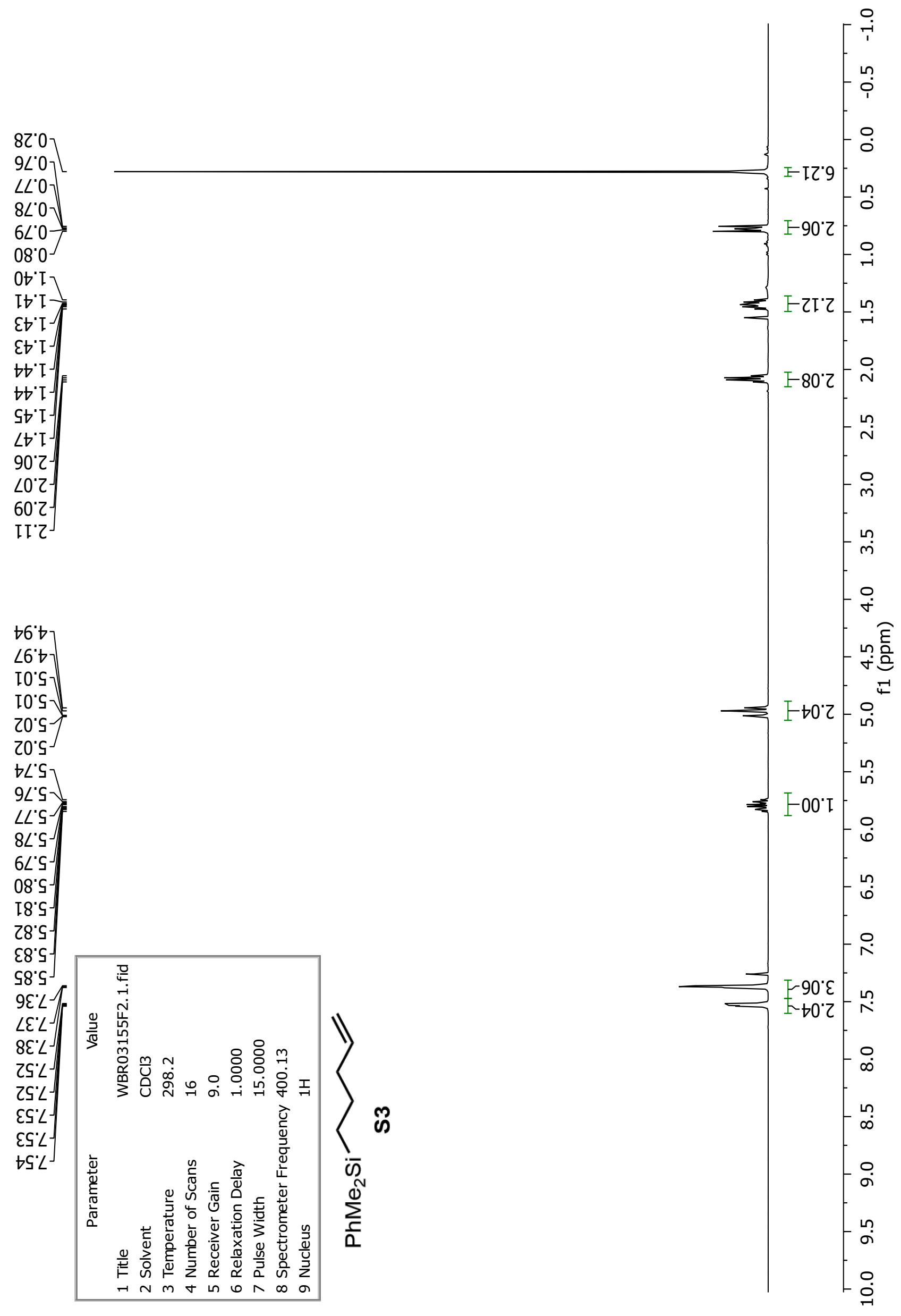


โ6'Z--

Et'SI-

IS'EZ-

$S L ' L \varepsilon-$

89ㄴII-

S8'LZ

\&6 $821-$

69'ยहI-

โ0.6हI

29.6हा
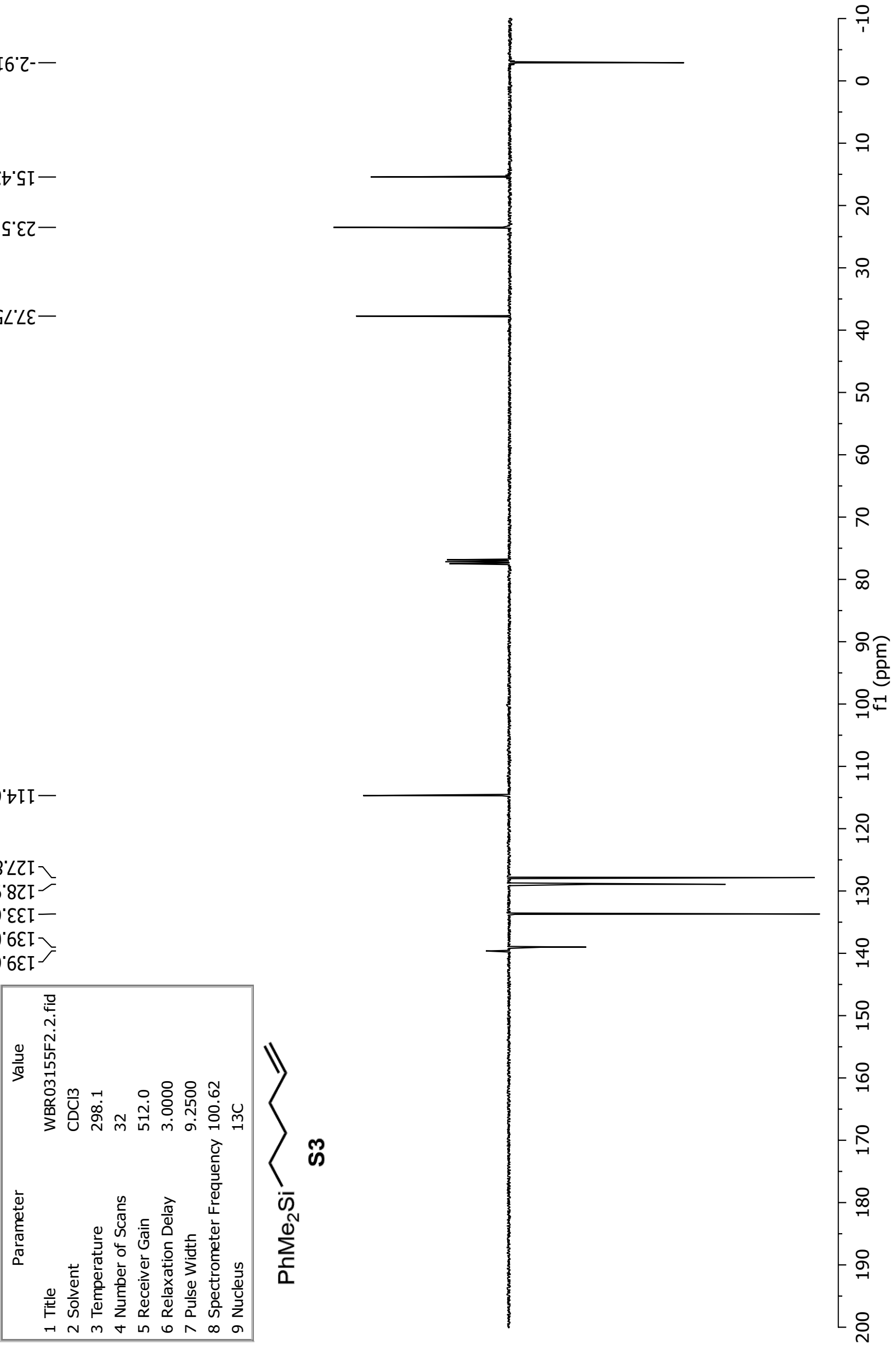


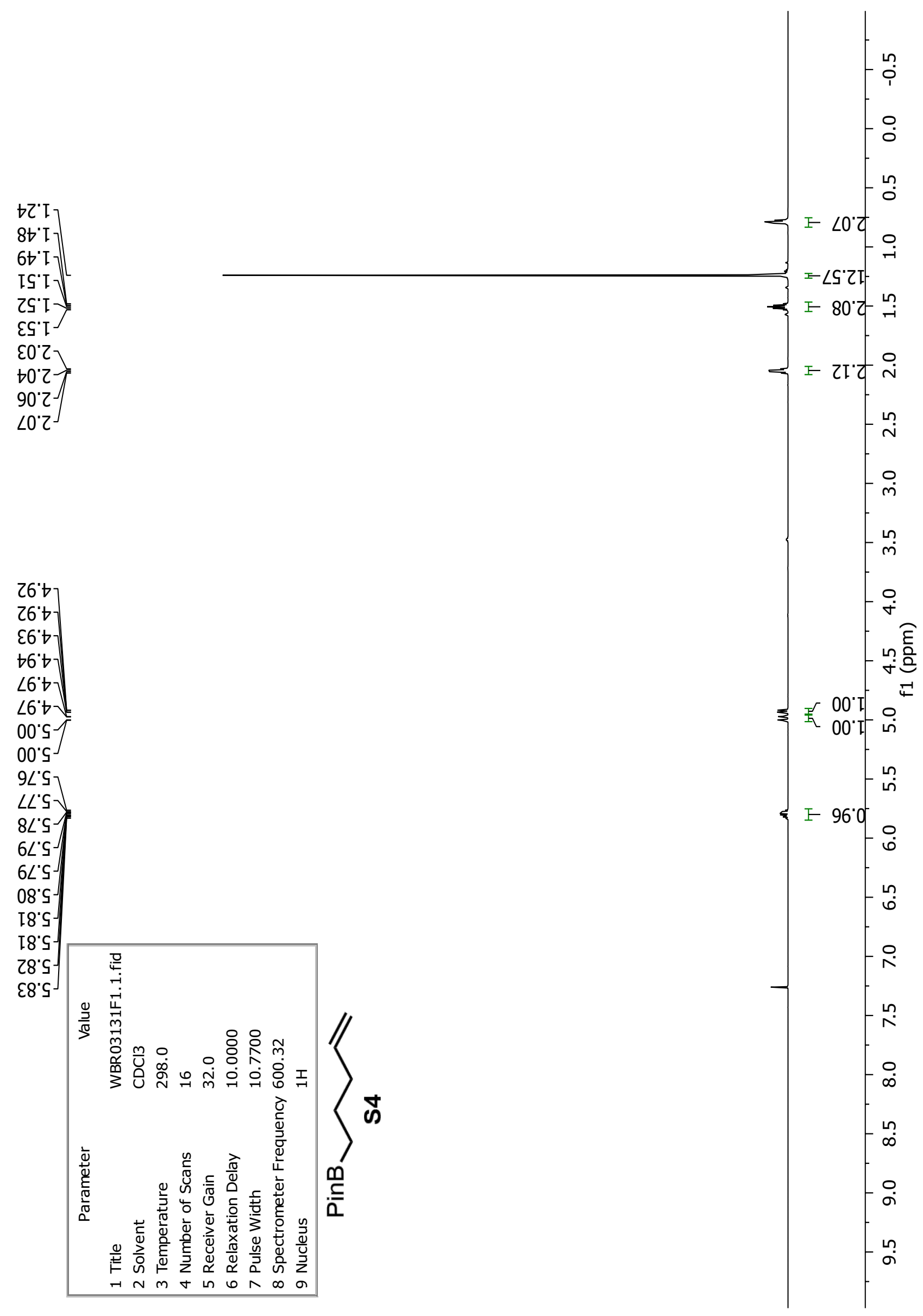



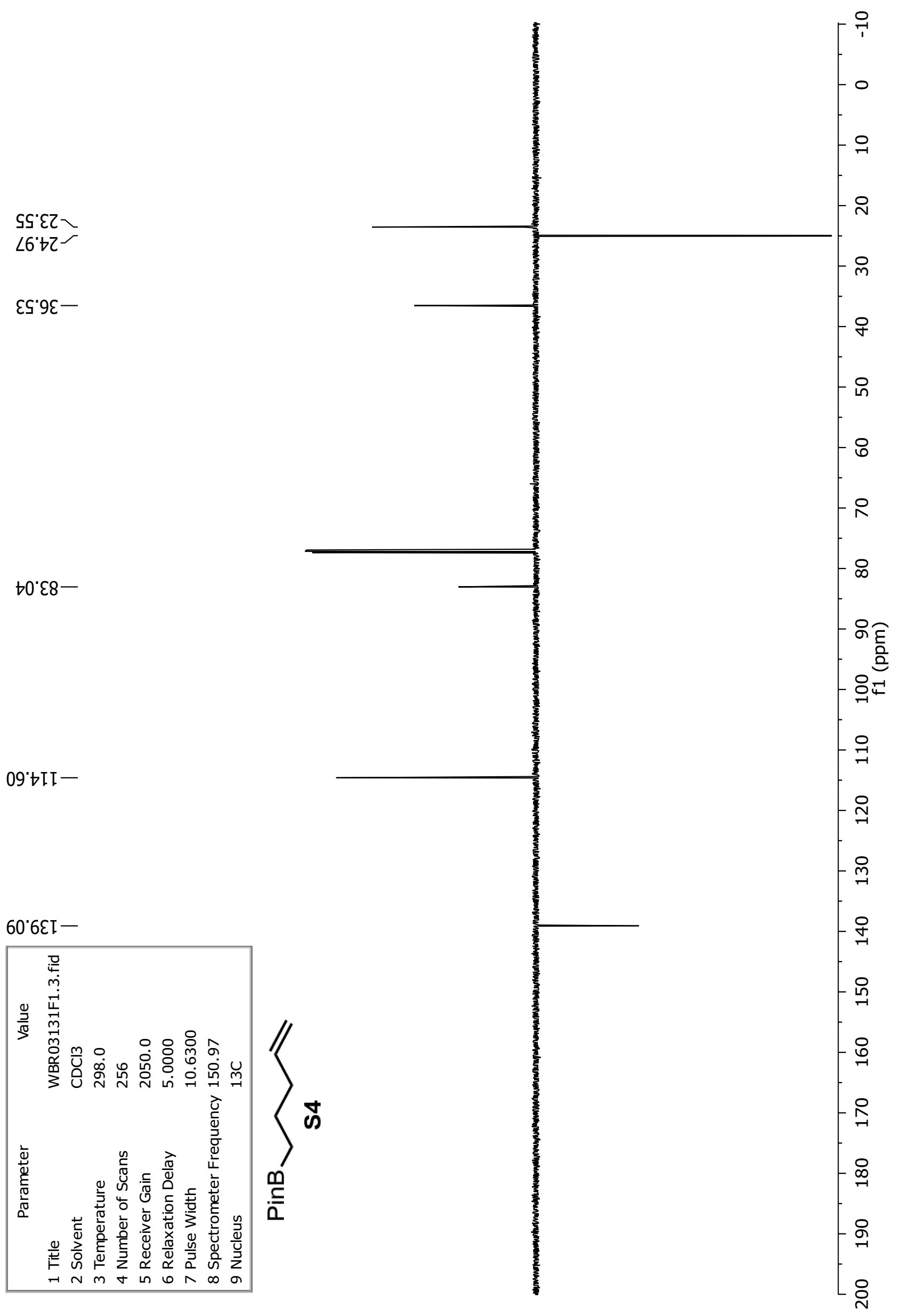

09ㄷI -

60.6EI-

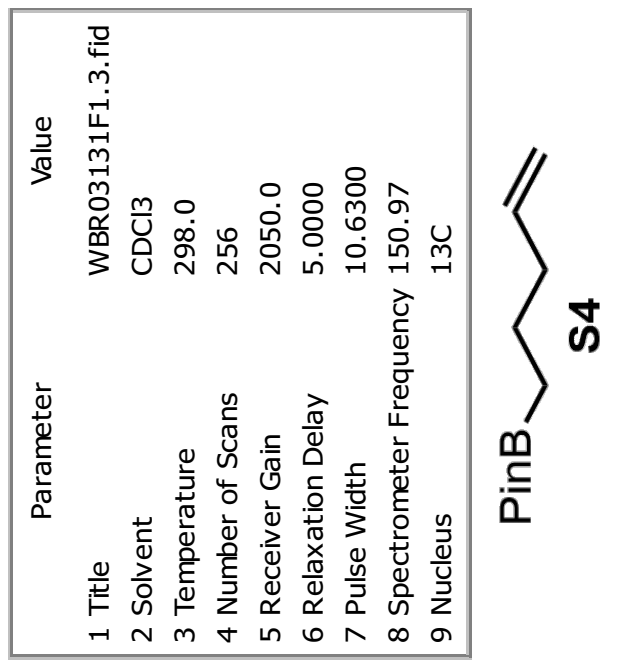




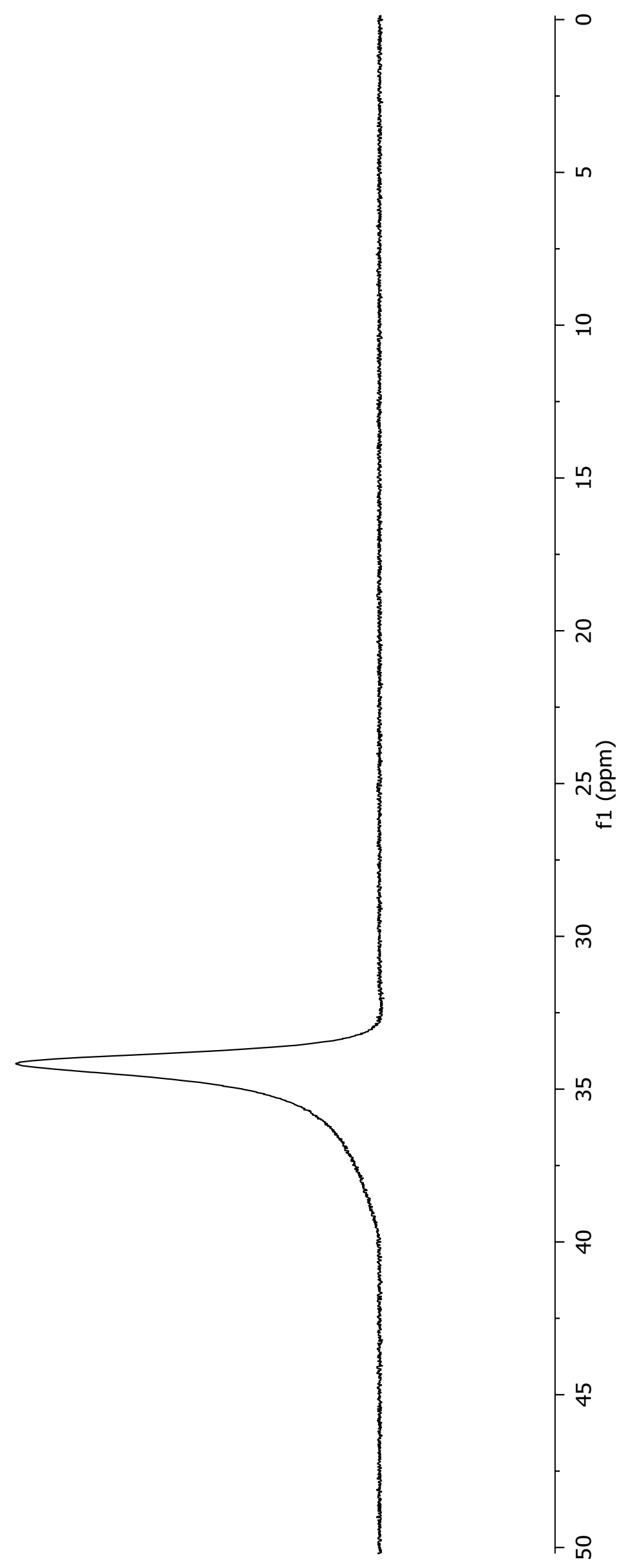




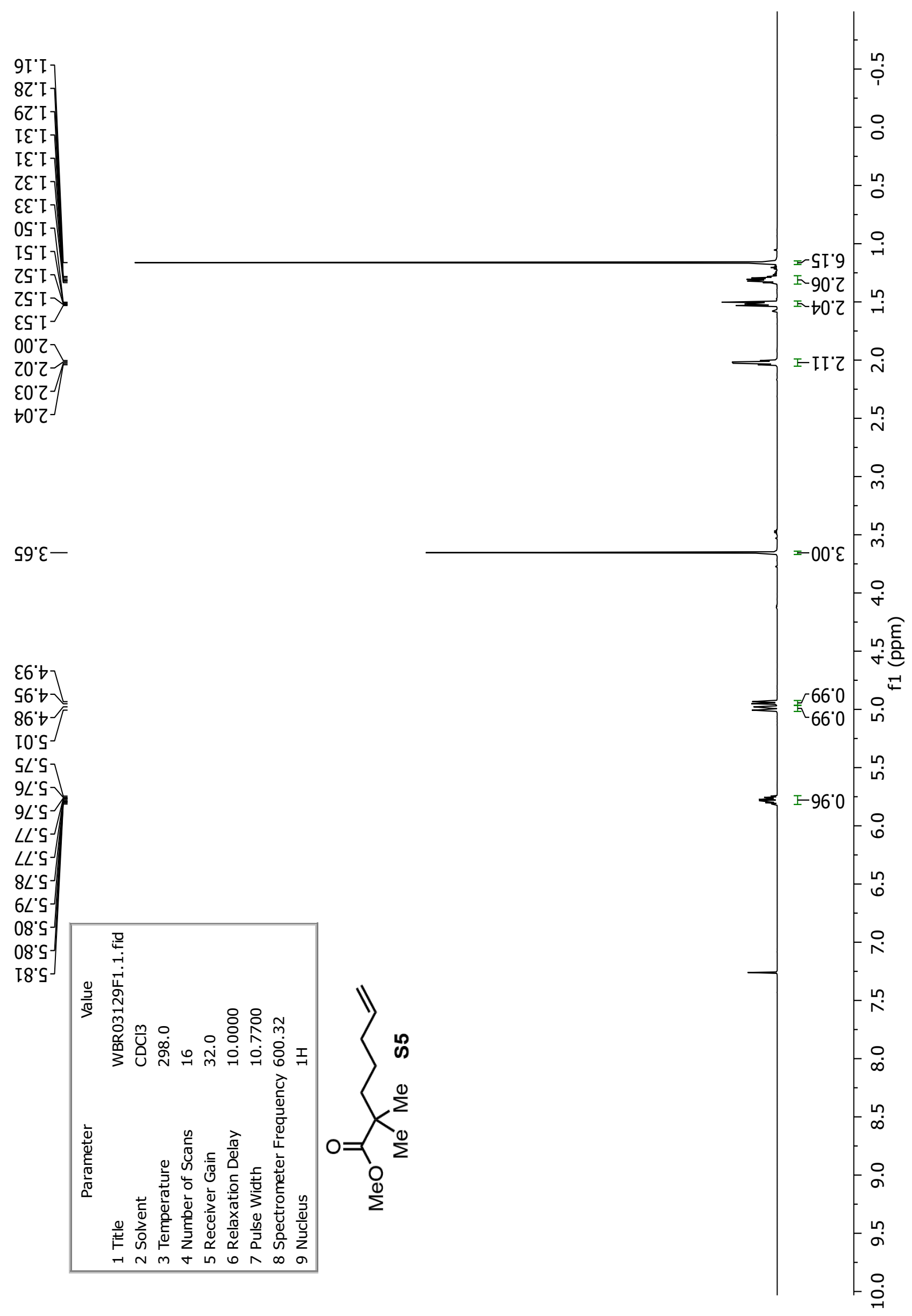



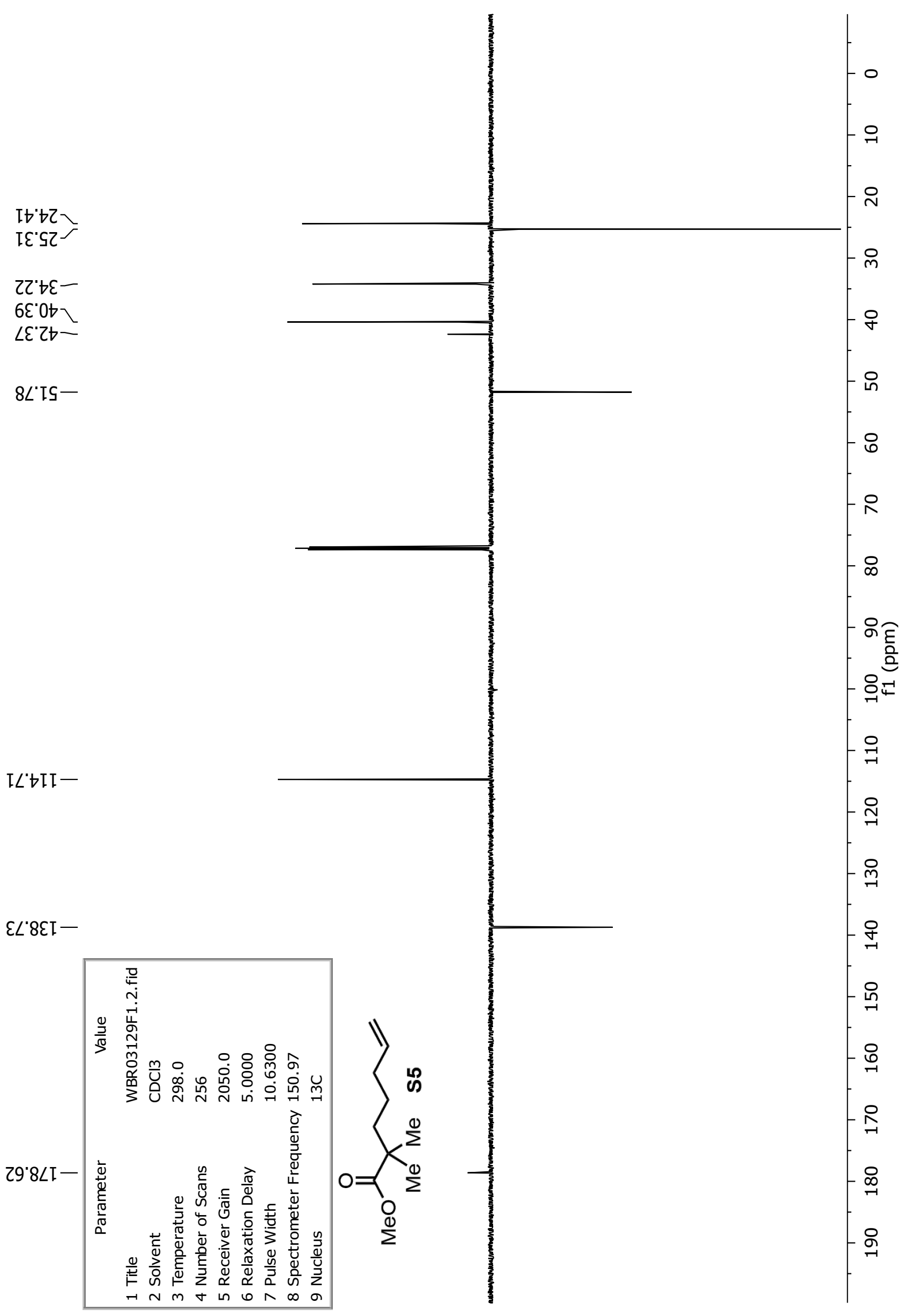


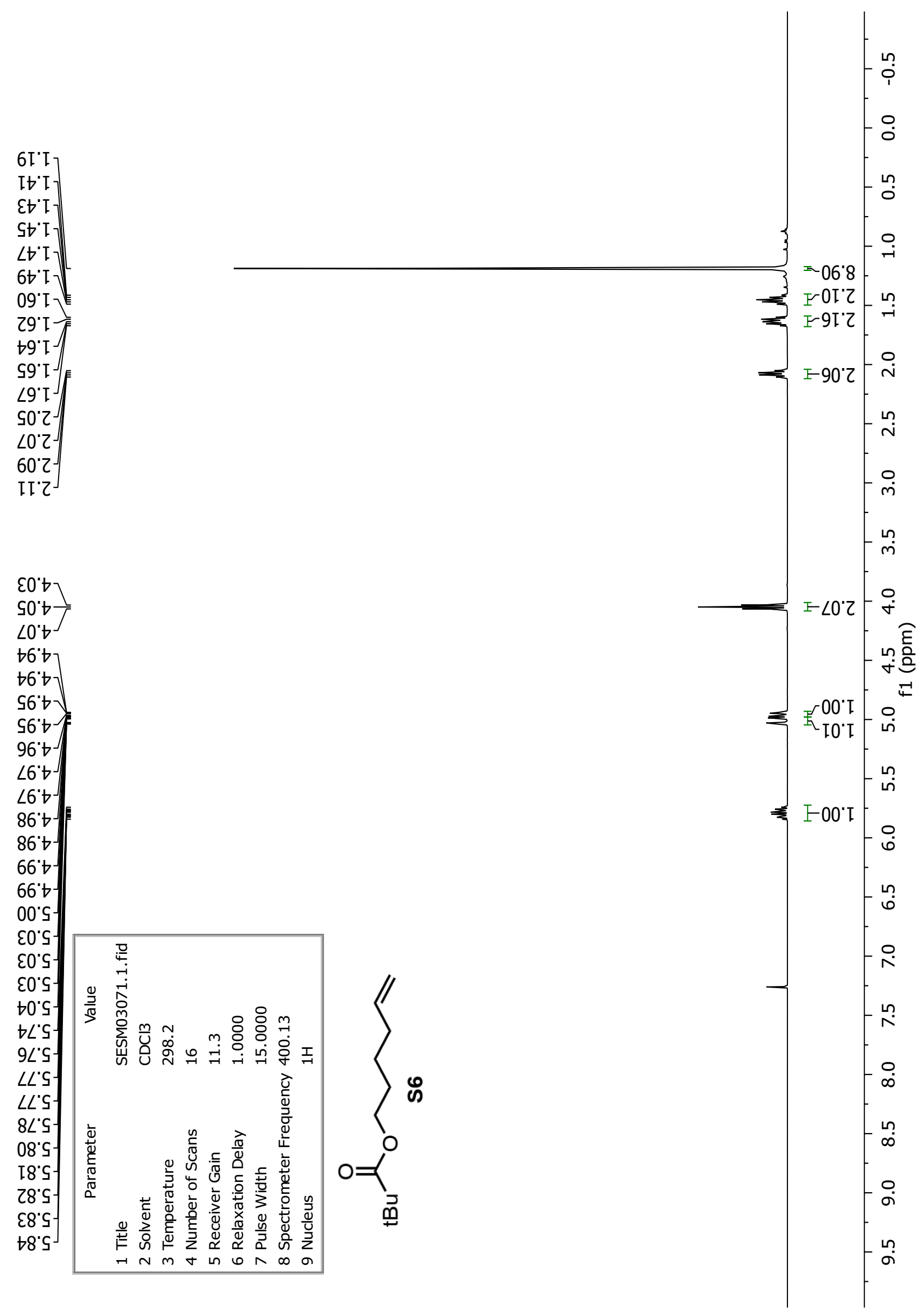




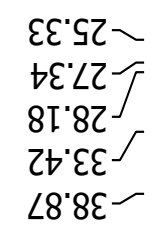

$9 \varepsilon ' เ 9-$

I6.tII-

SS'8EI-

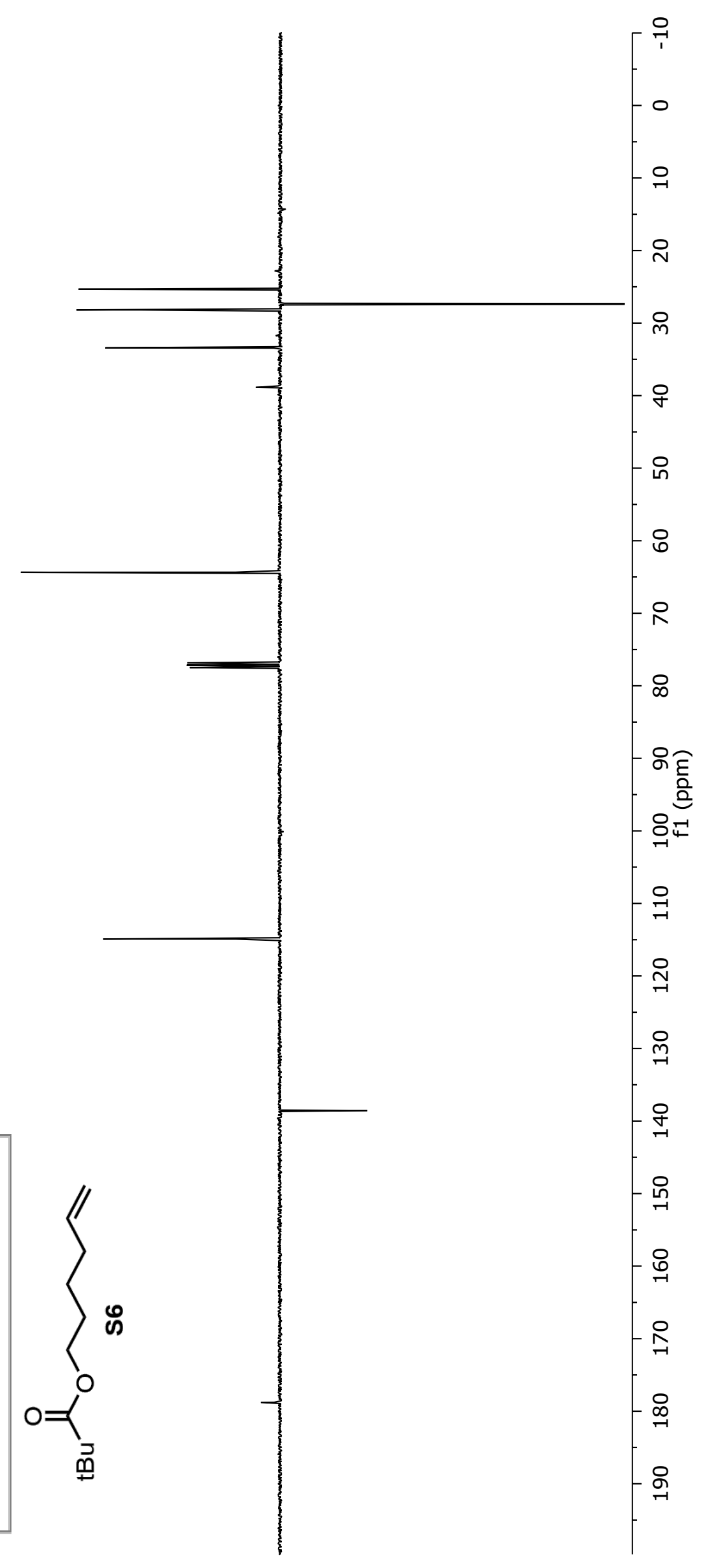




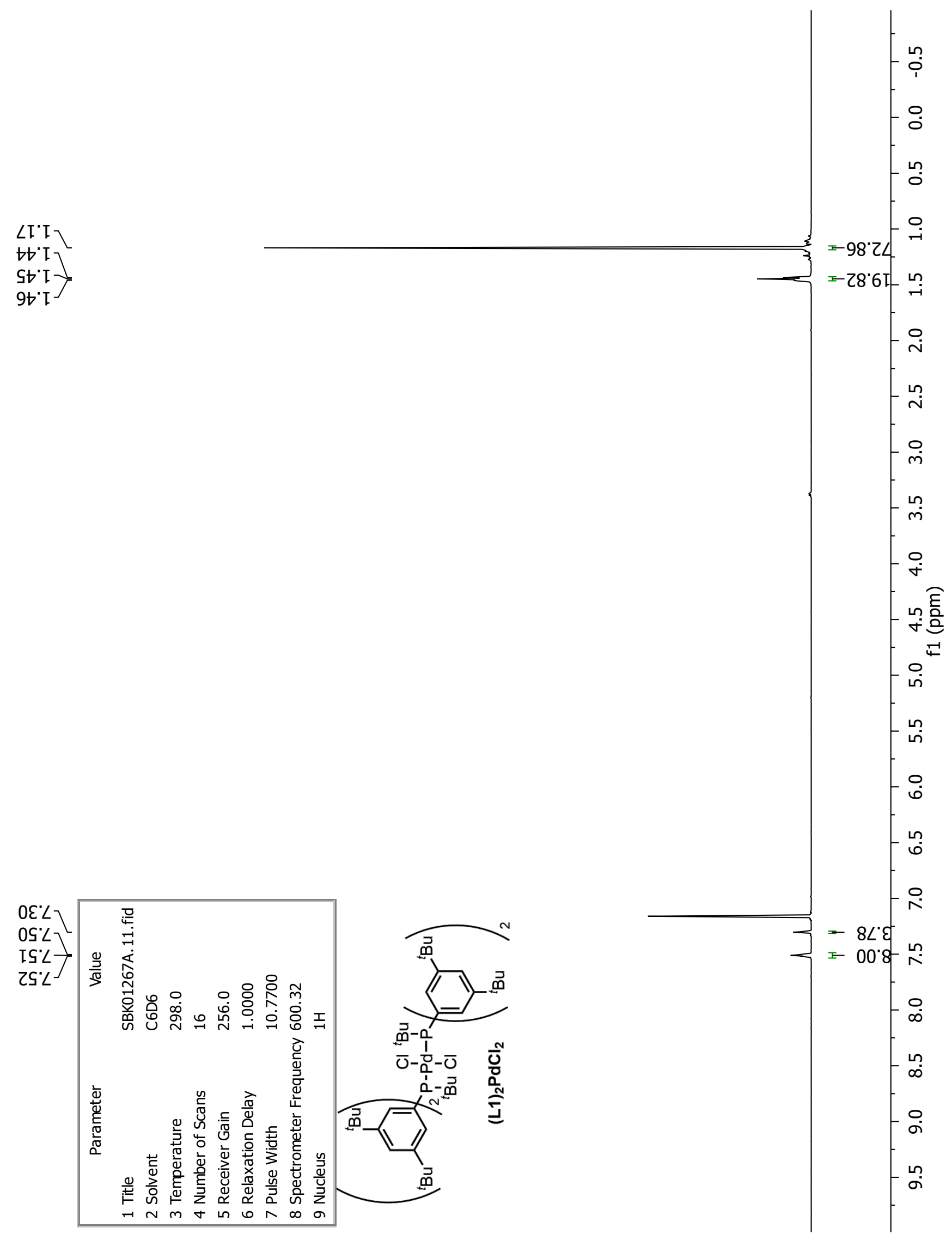




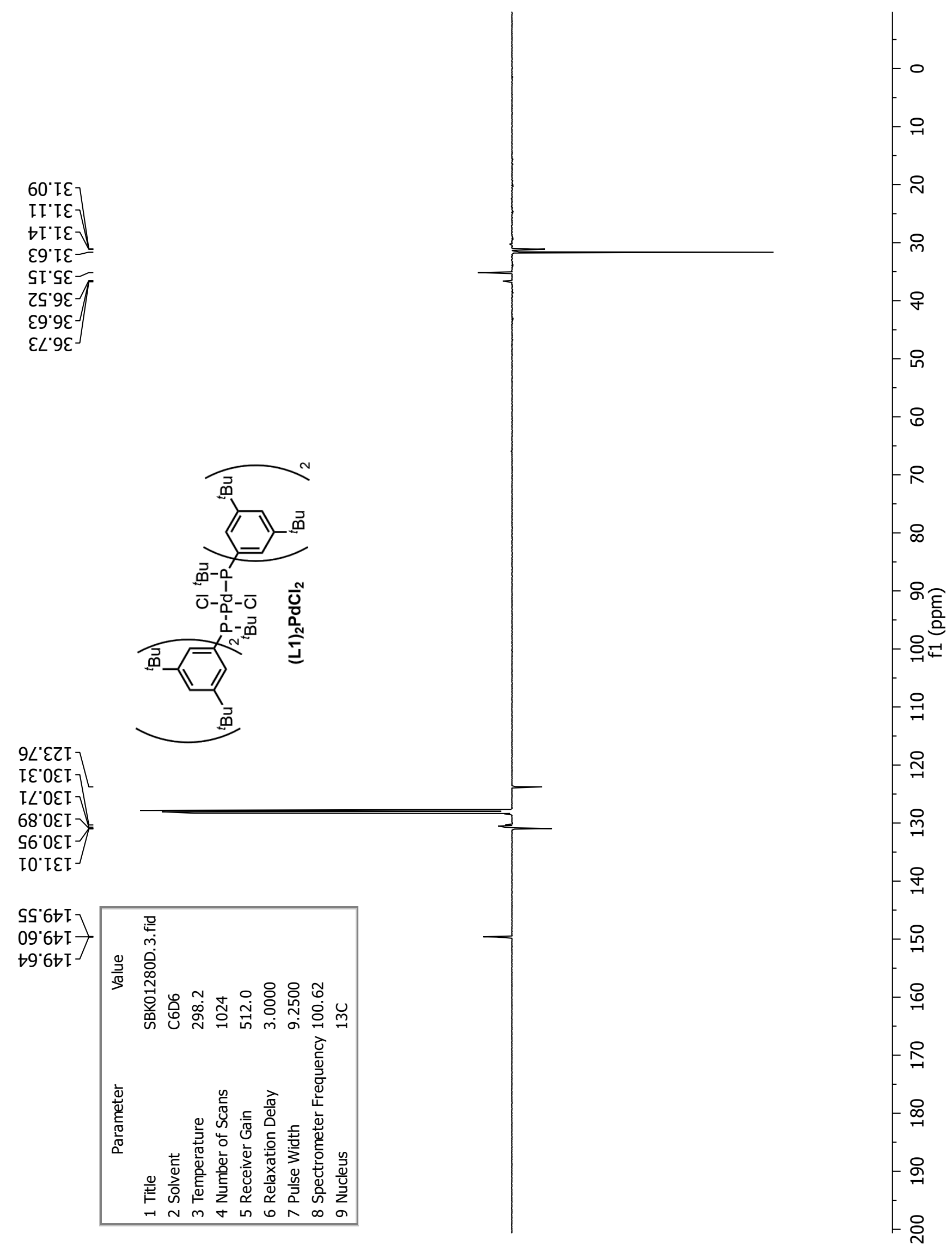




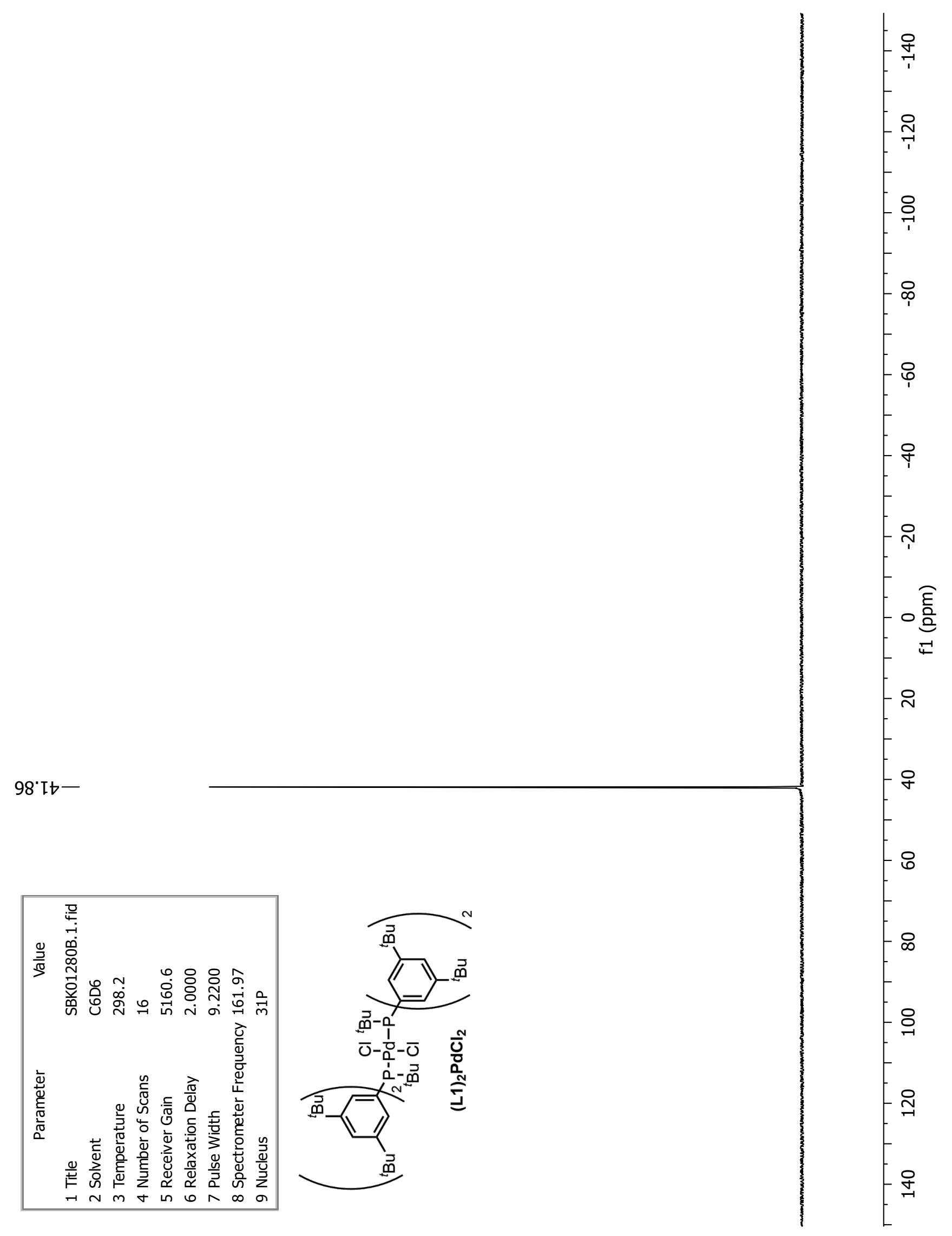




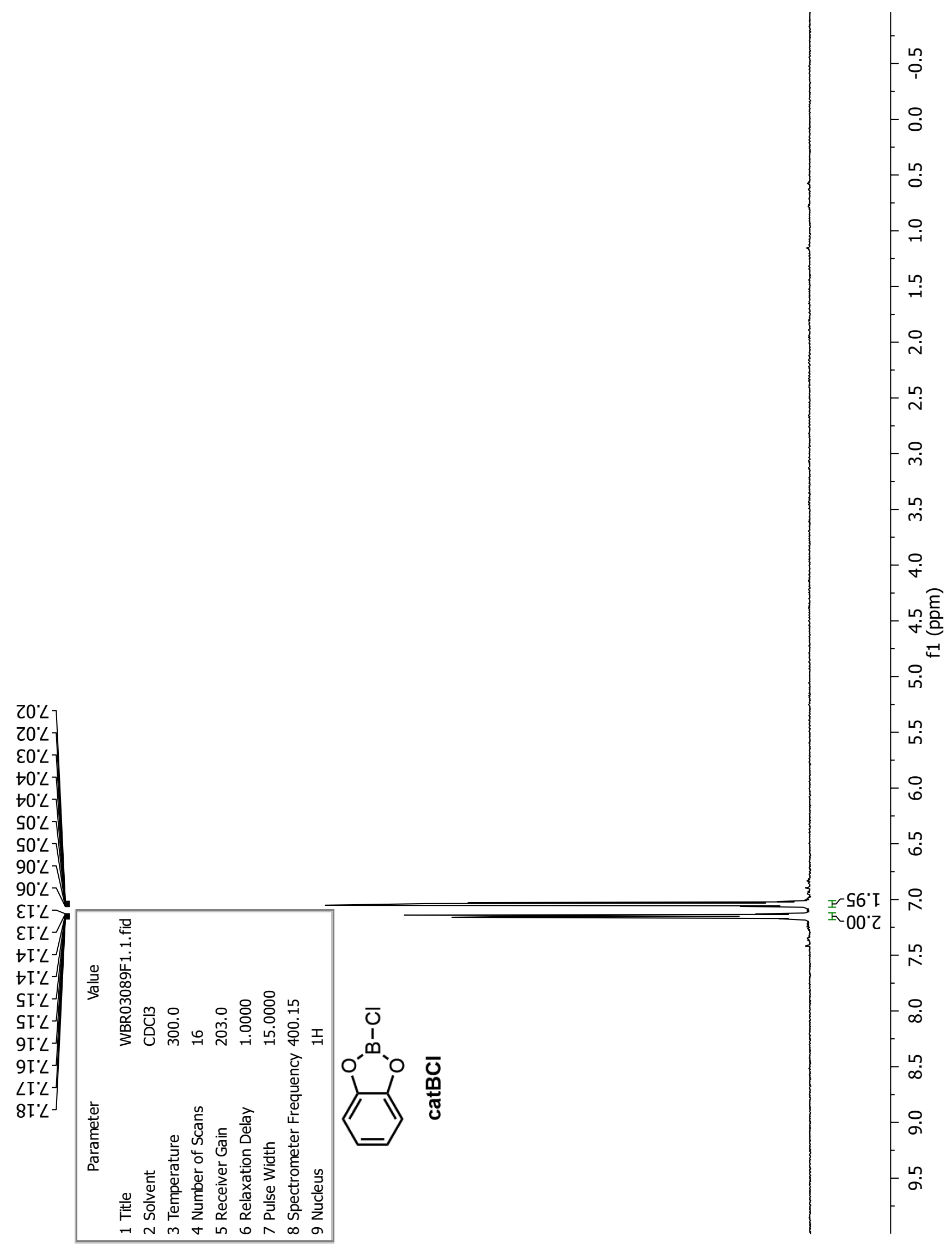




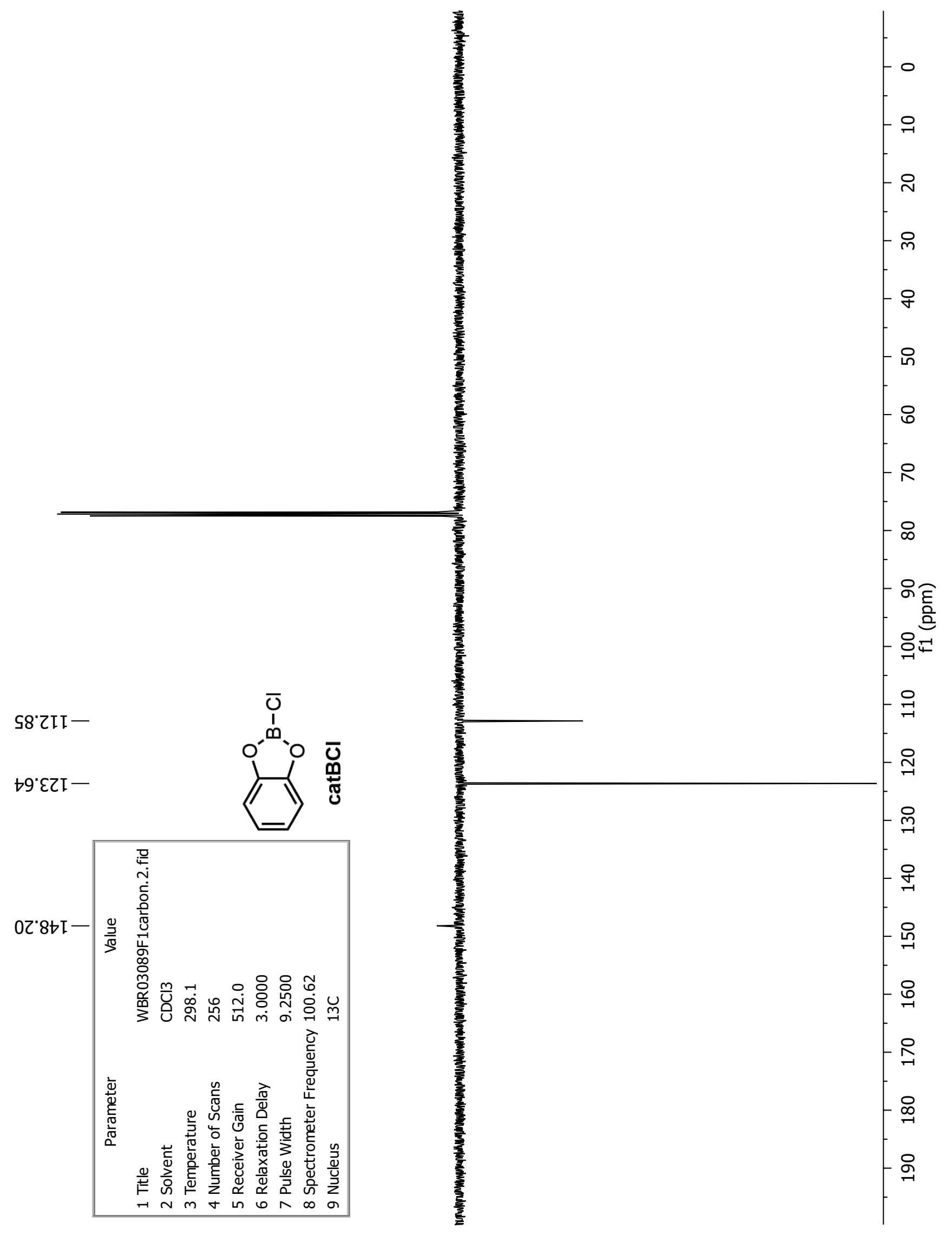



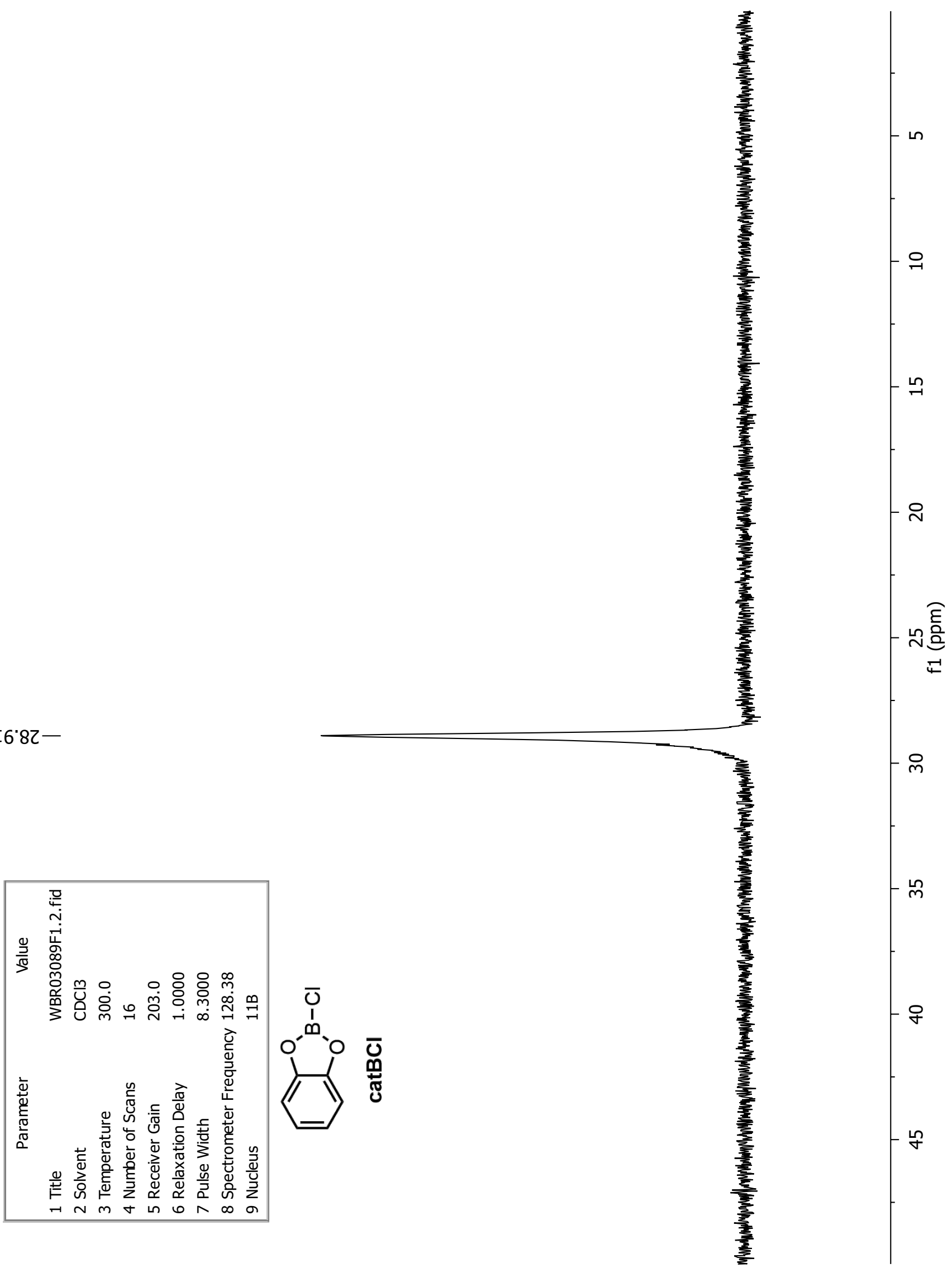


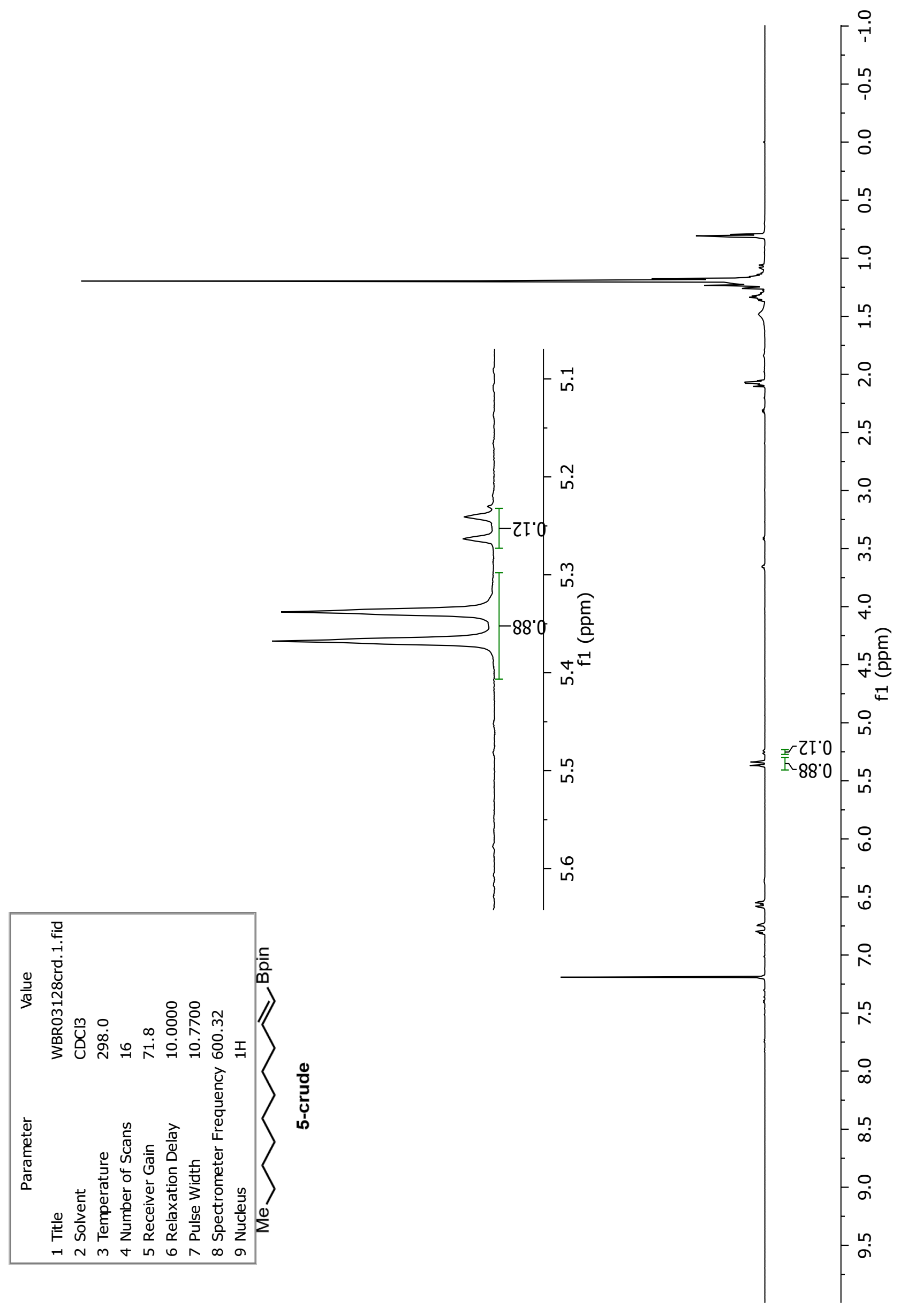




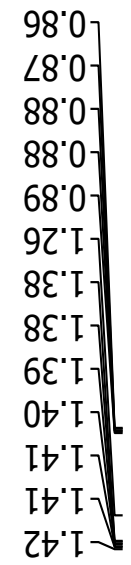

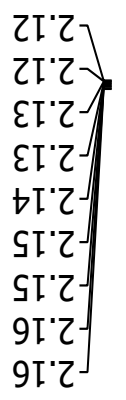
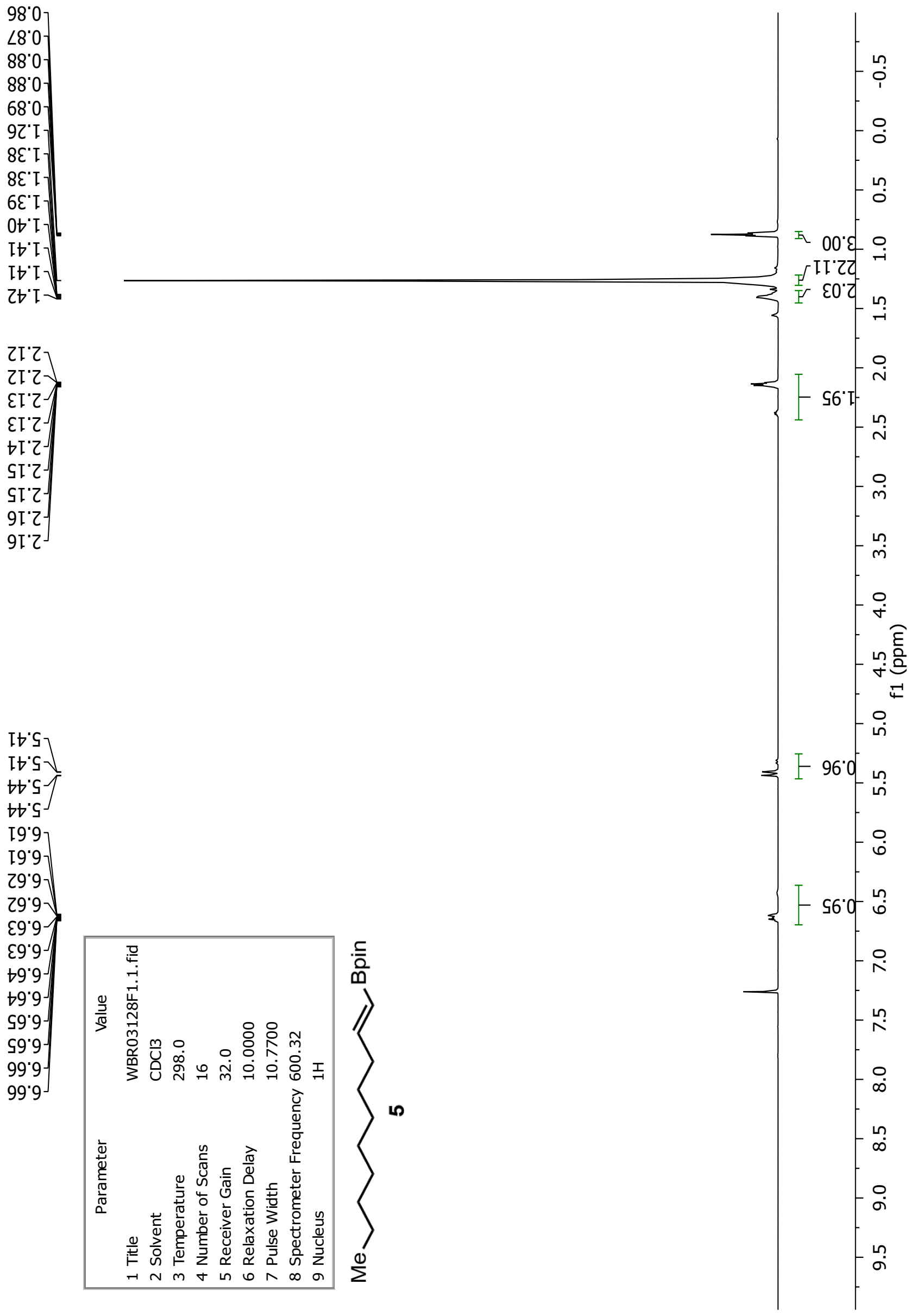


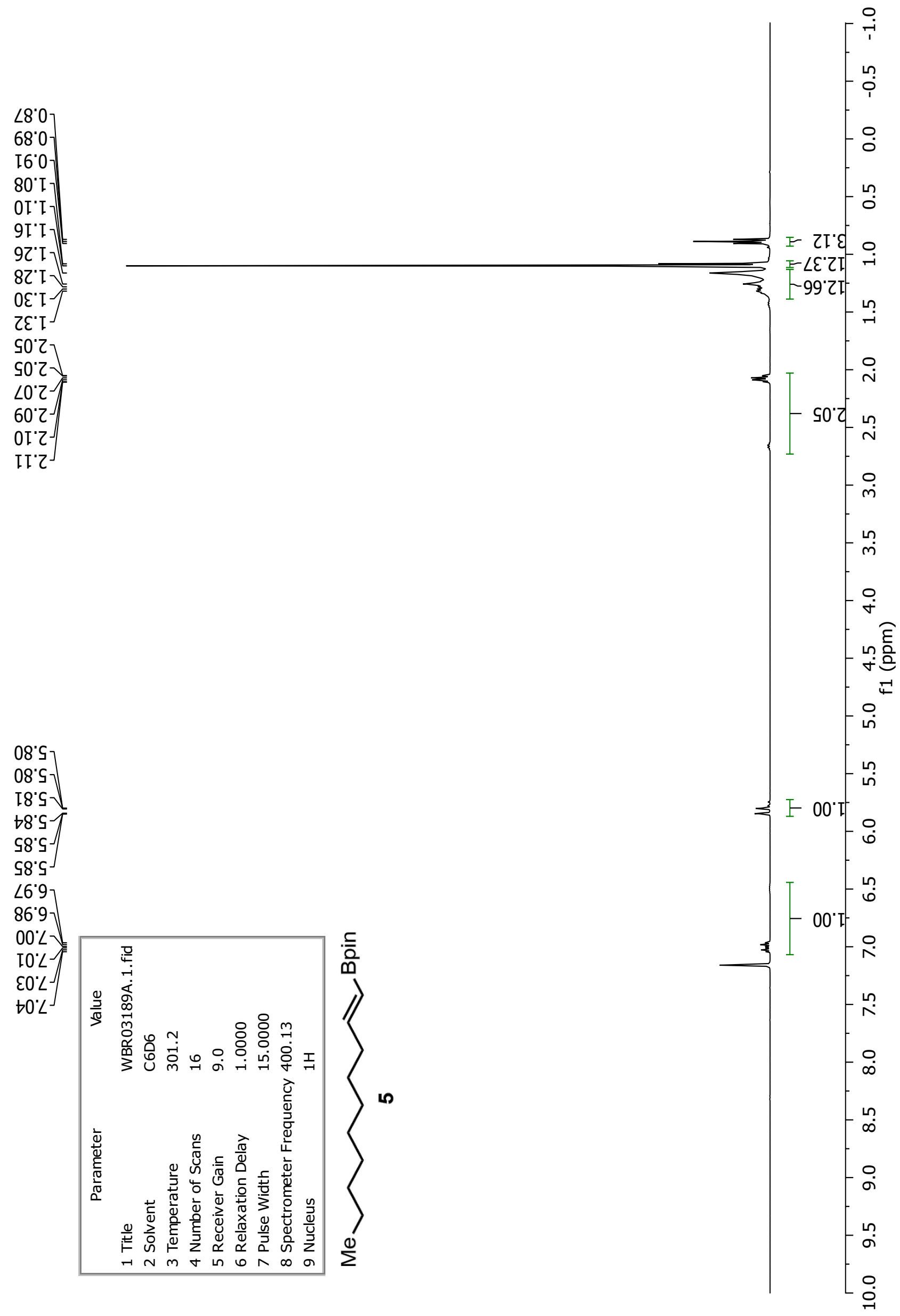


9Z๋เ 一

$\varepsilon 8.22$

$+66^{\circ}+2$

$8 \varepsilon^{\prime} 82$ -

$0 t^{\circ} 62-$

โ9'6z]

เ0'حع

$00^{\circ} 9 \varepsilon$

乙I'ย8-

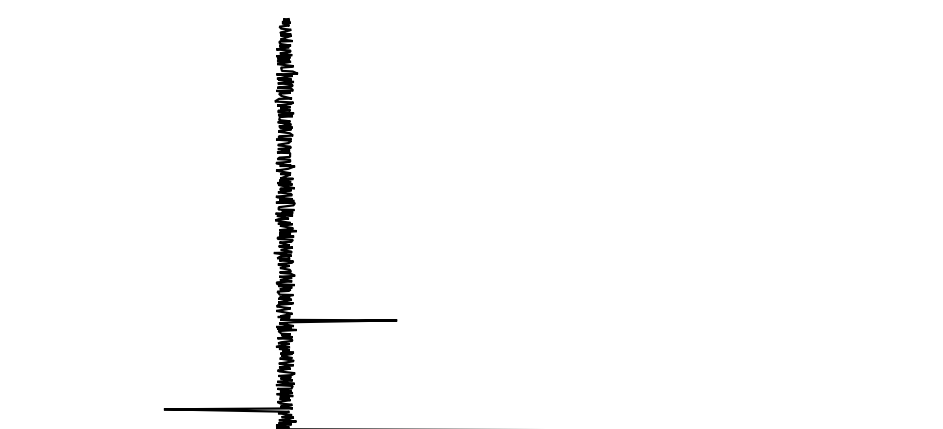

$-\frac{0}{1}$

o

음

ำ

m

우

오

8

옹

$\infty$

8

을

$^{7}$

.

윽

윽

움

욱

$-8$

옥

$\infty$

욱

우

읏 


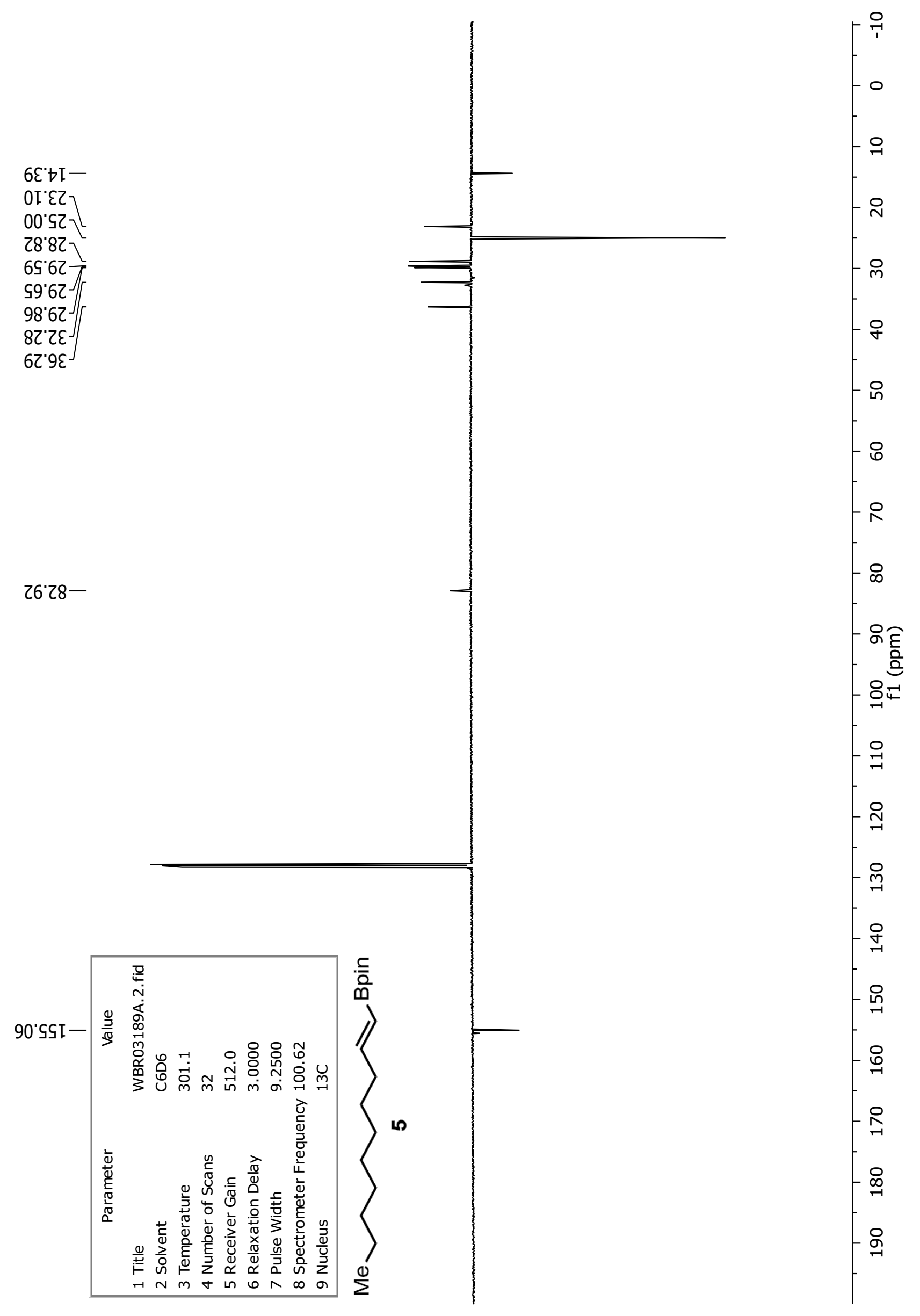




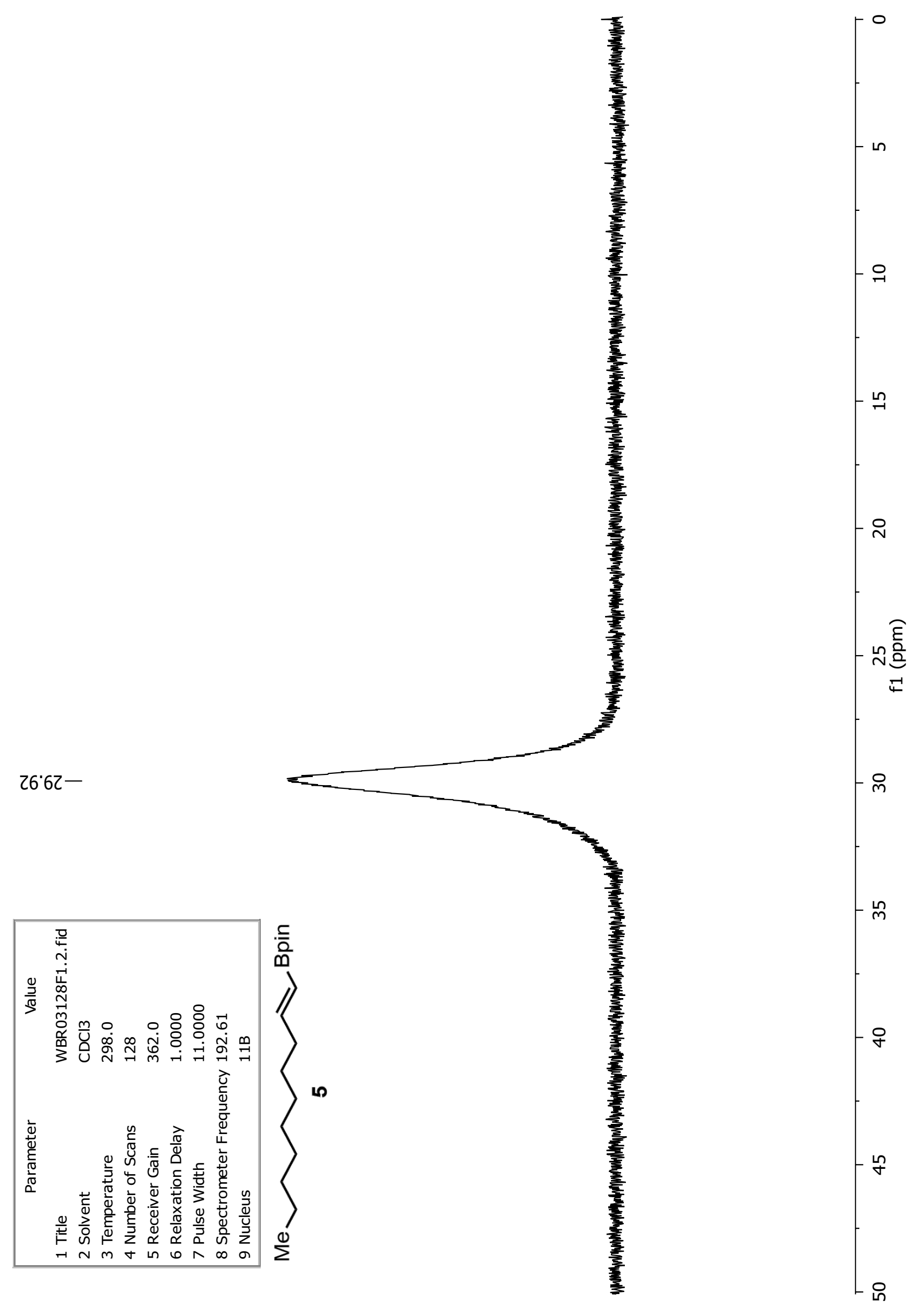




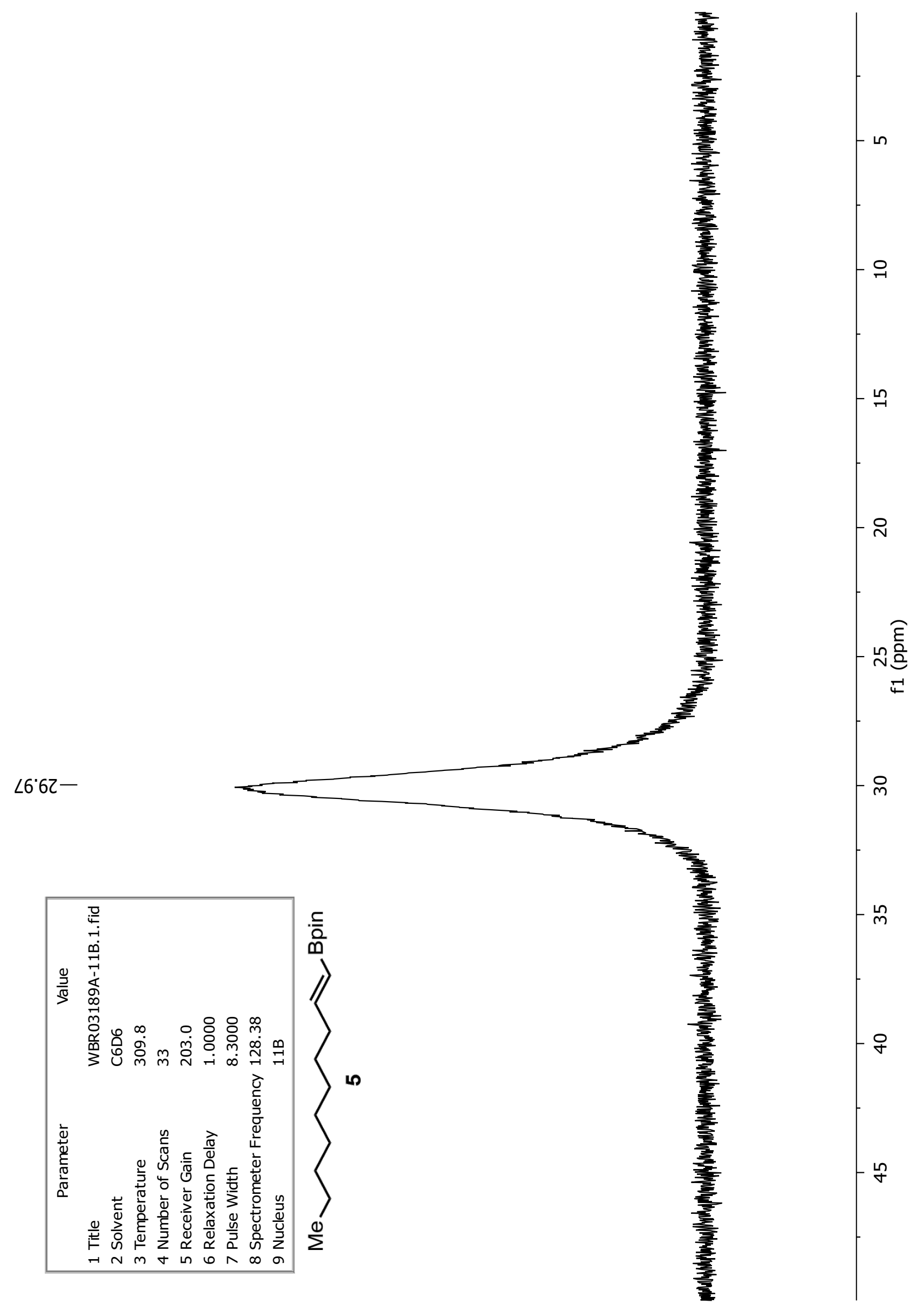




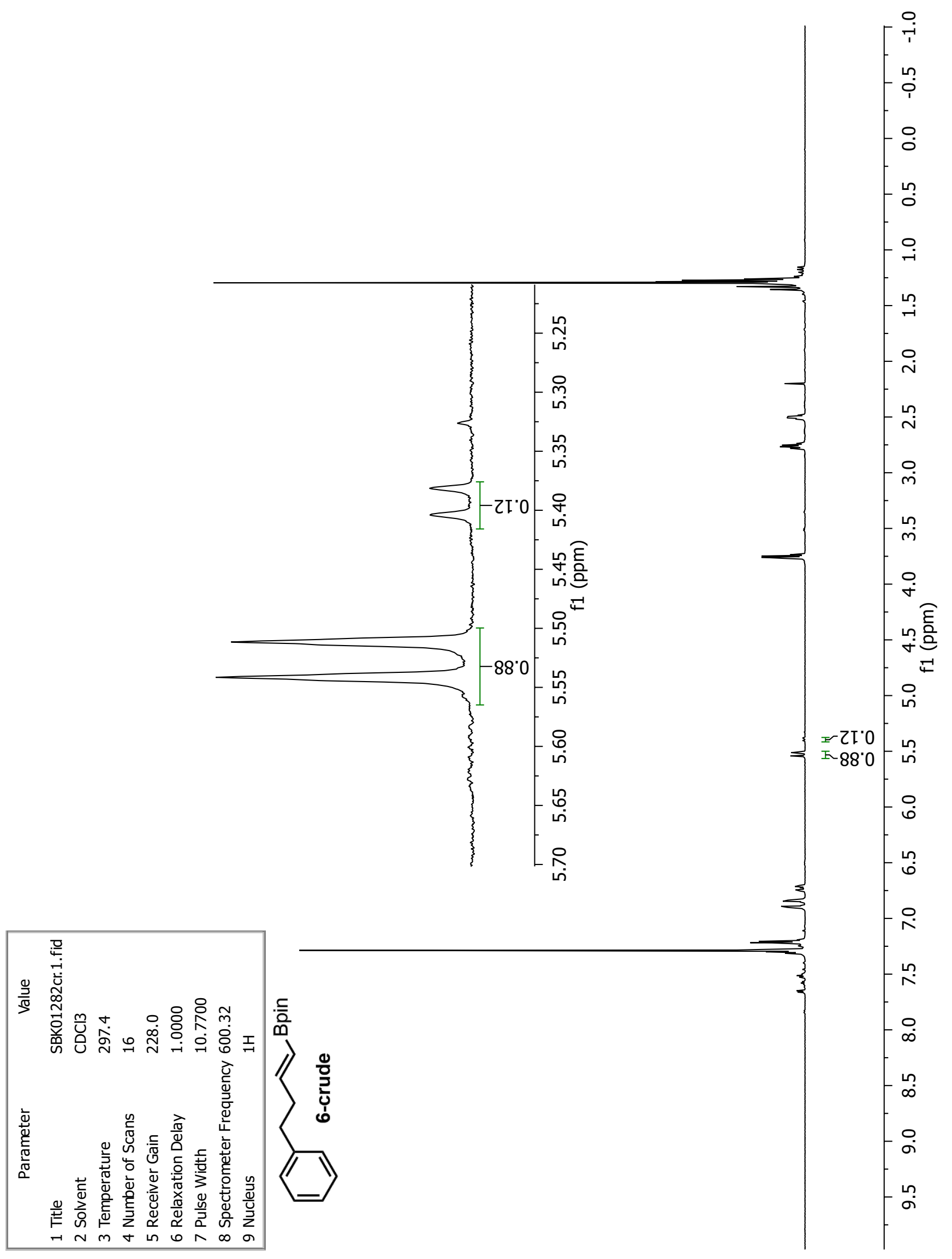




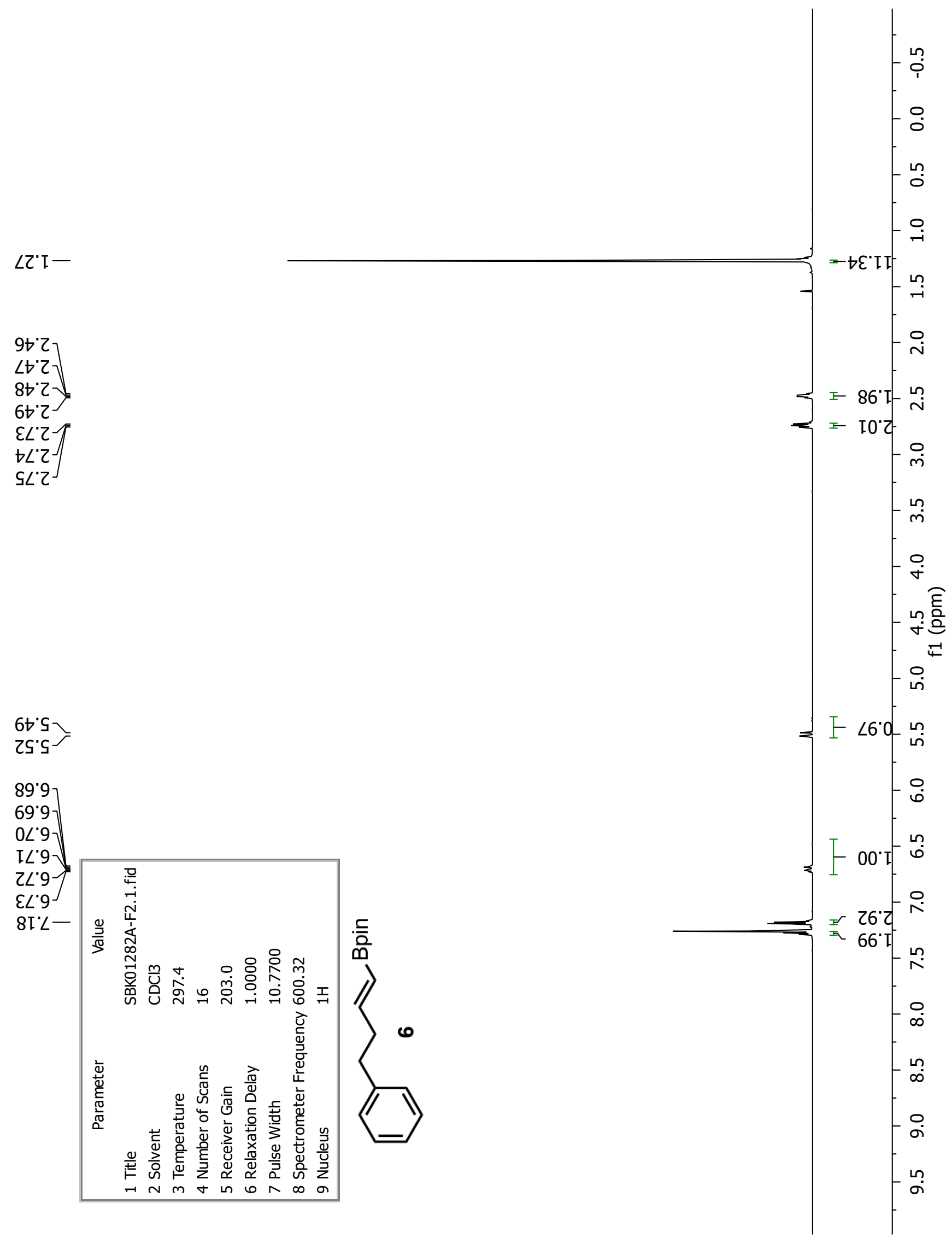




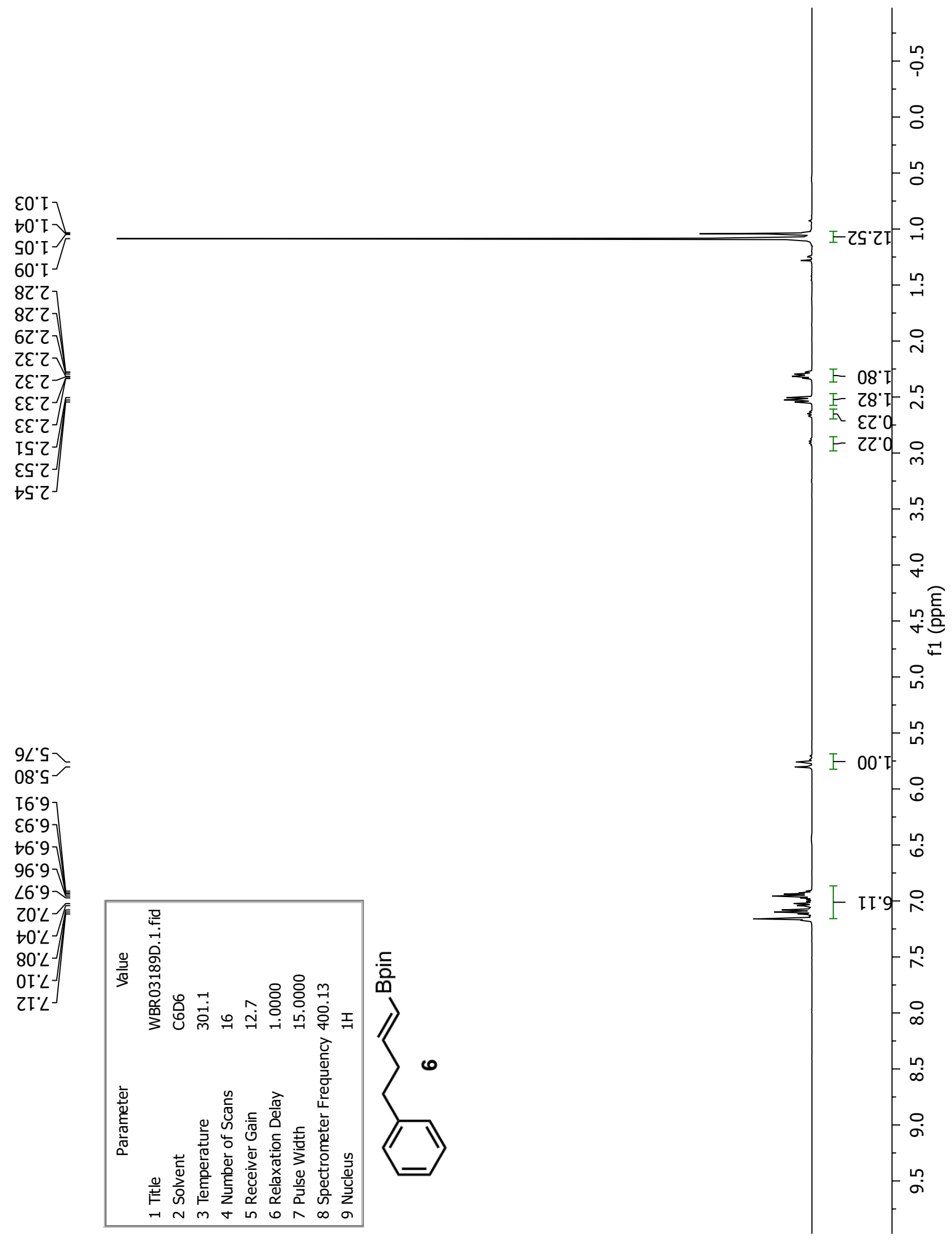


$26 \circ 2$

$0 \angle\llcorner\downarrow \varepsilon-$

$S 9^{\circ} \angle \mathcal{E}-$

乙૮'ย8-

86.SZI -

$9 b^{\circ} 82 \mathrm{I}$ -

06. It I -

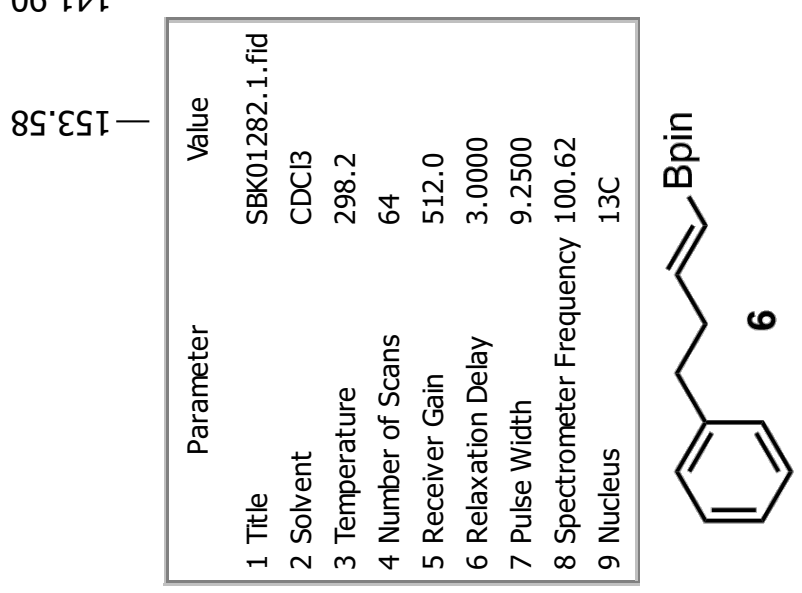

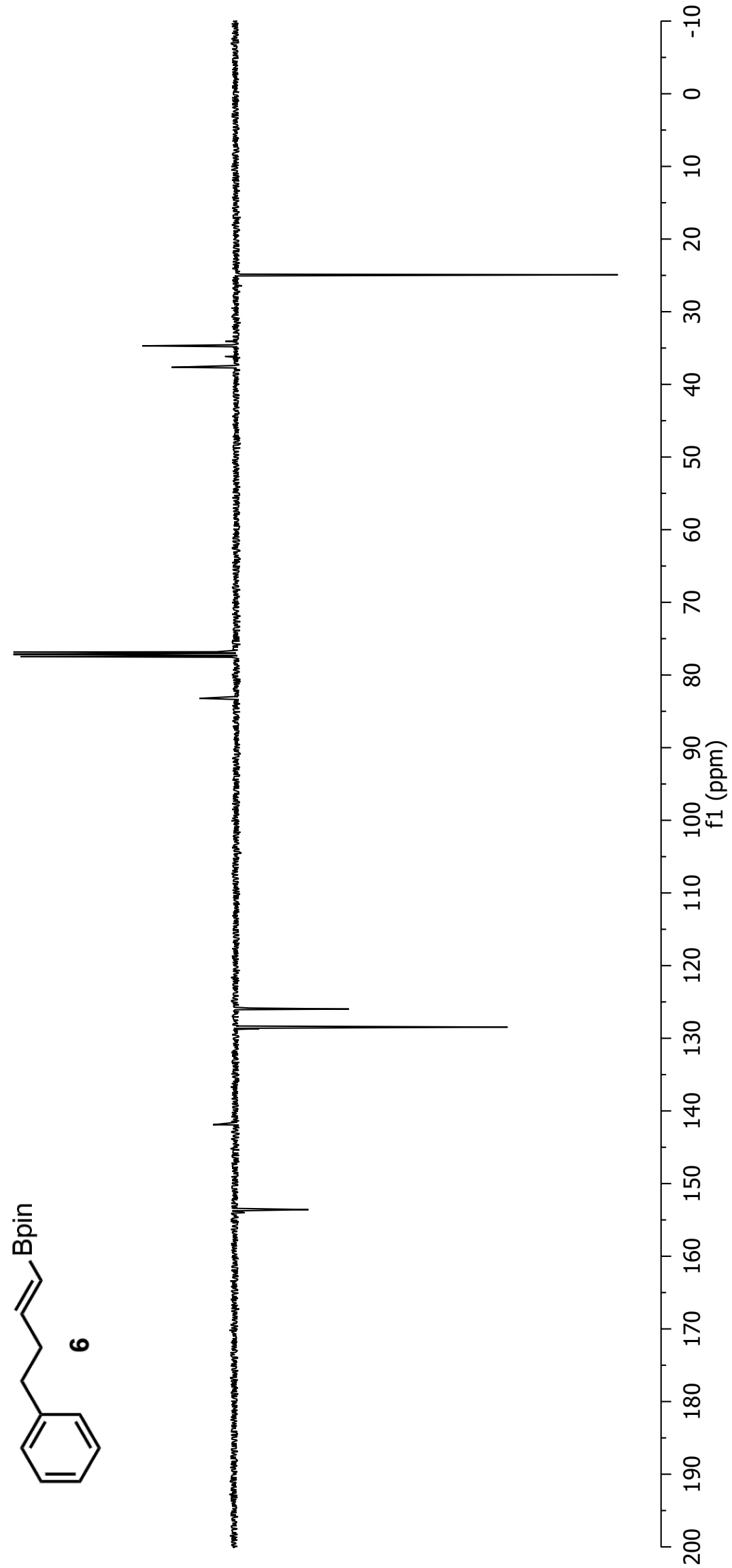

우 


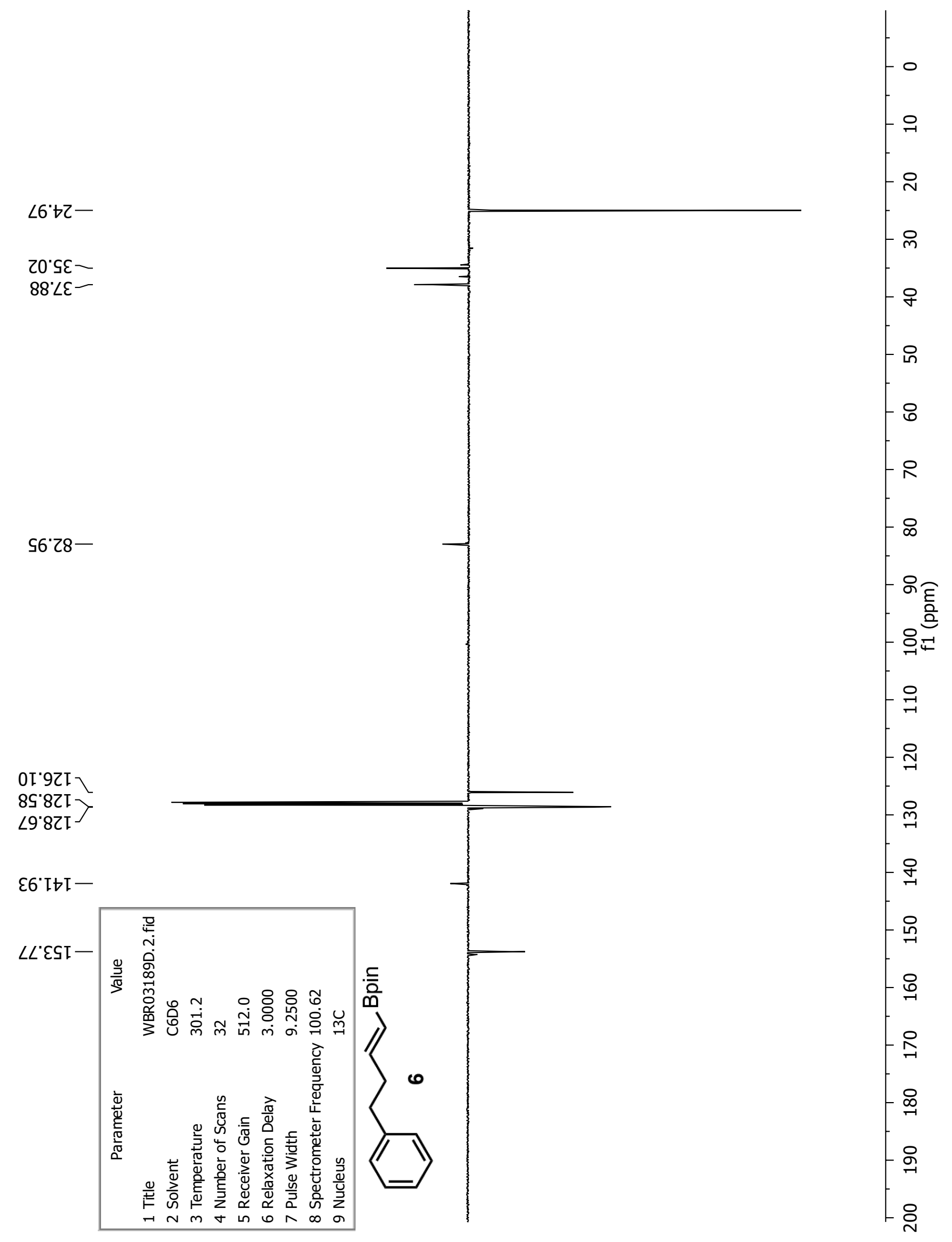




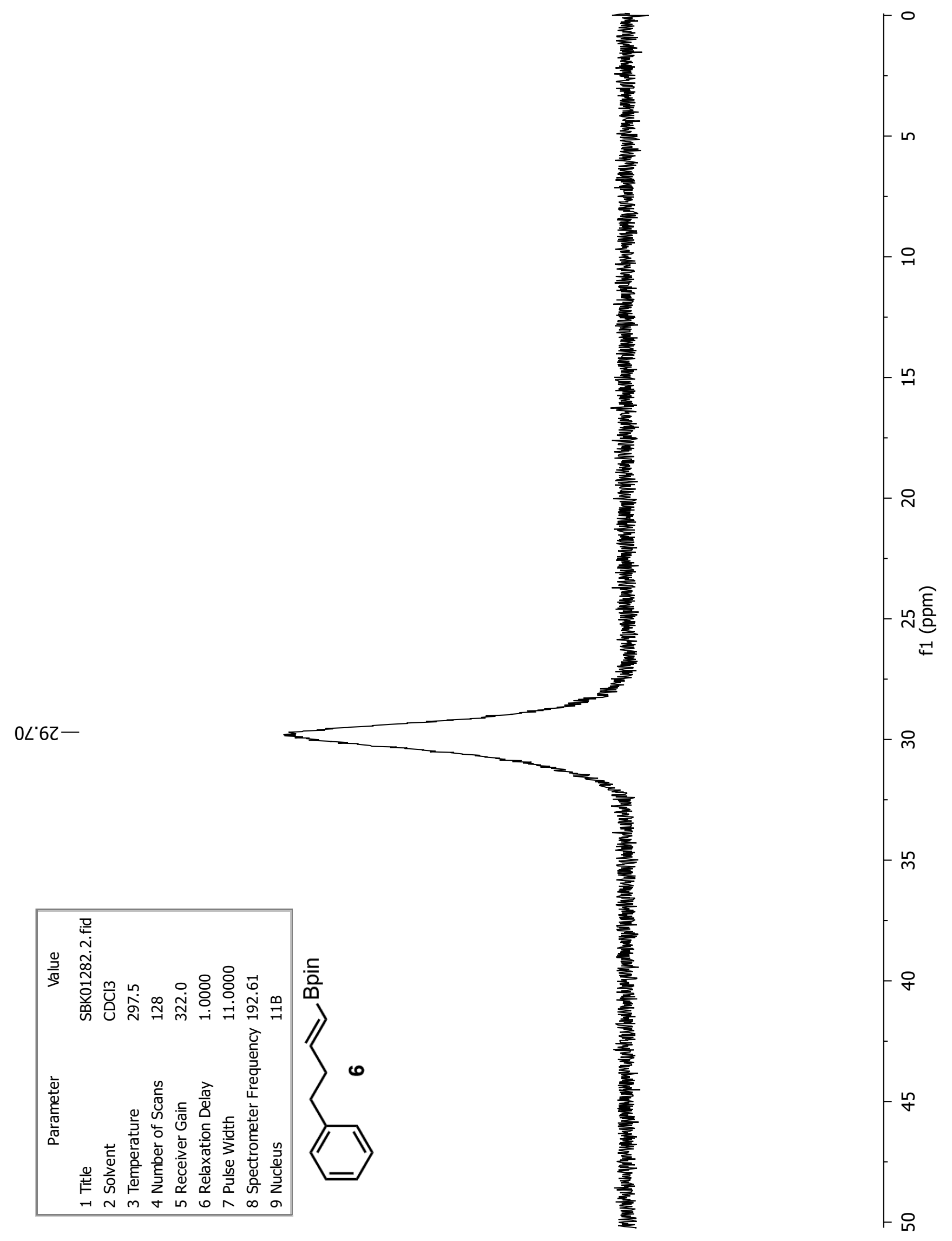




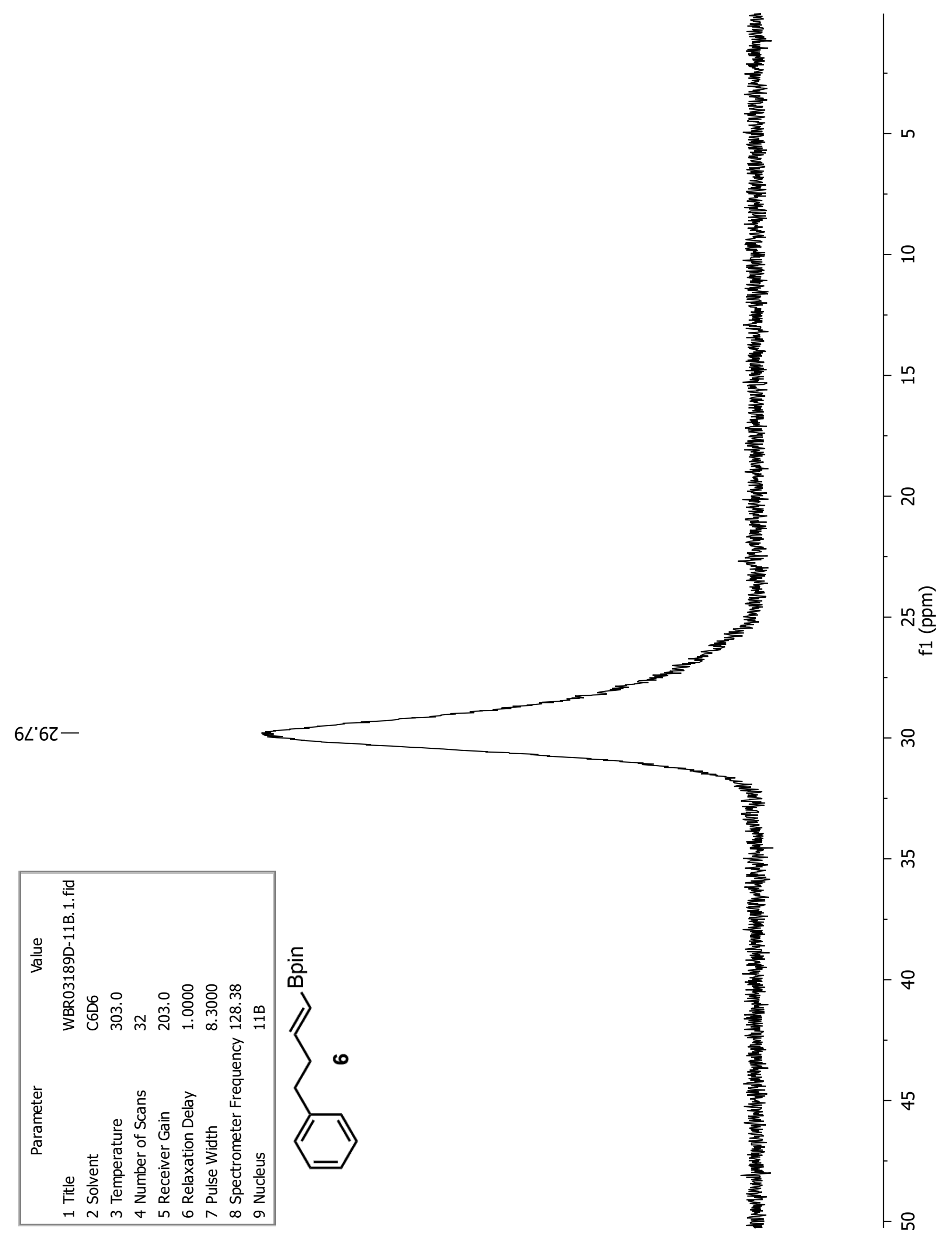




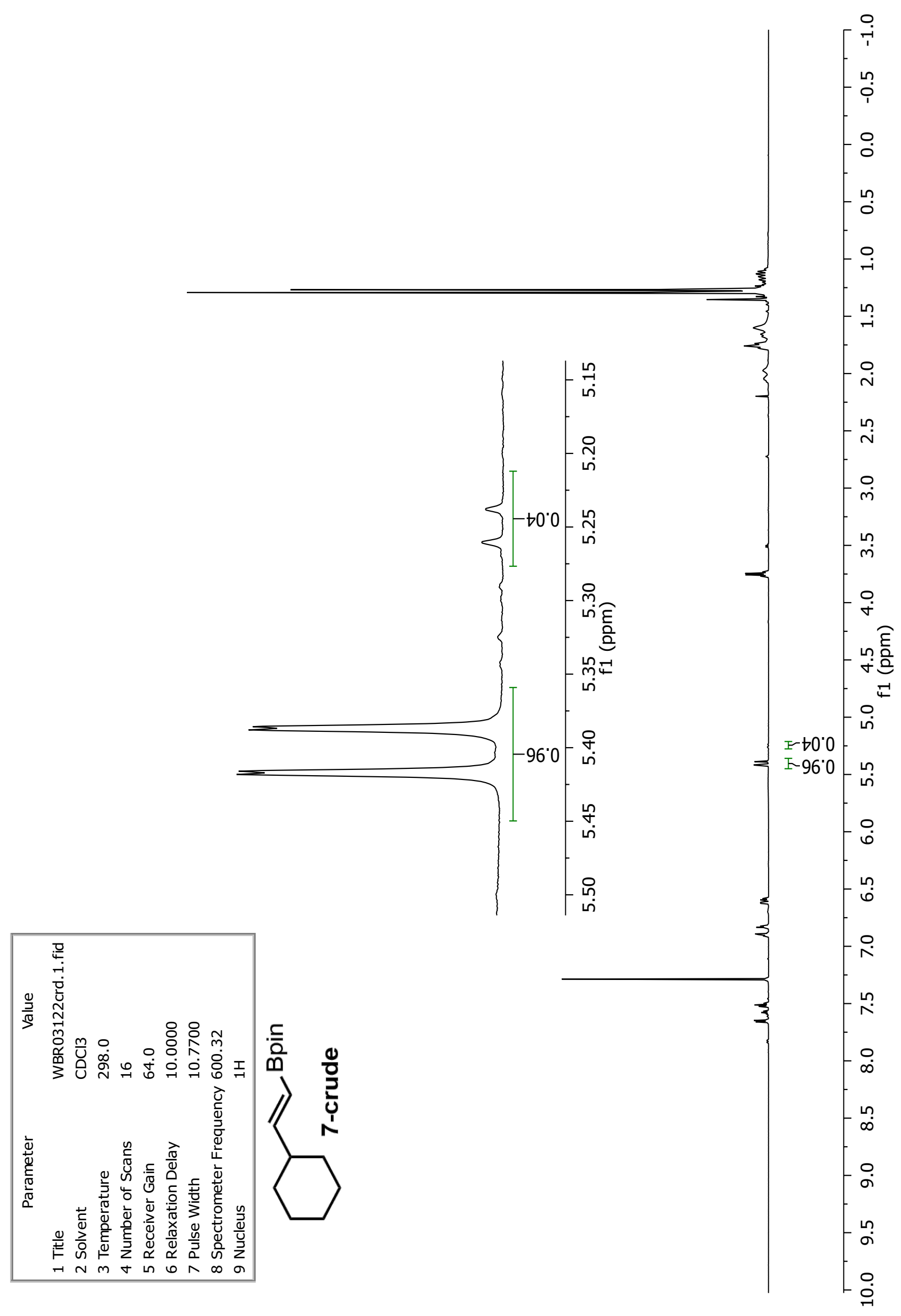




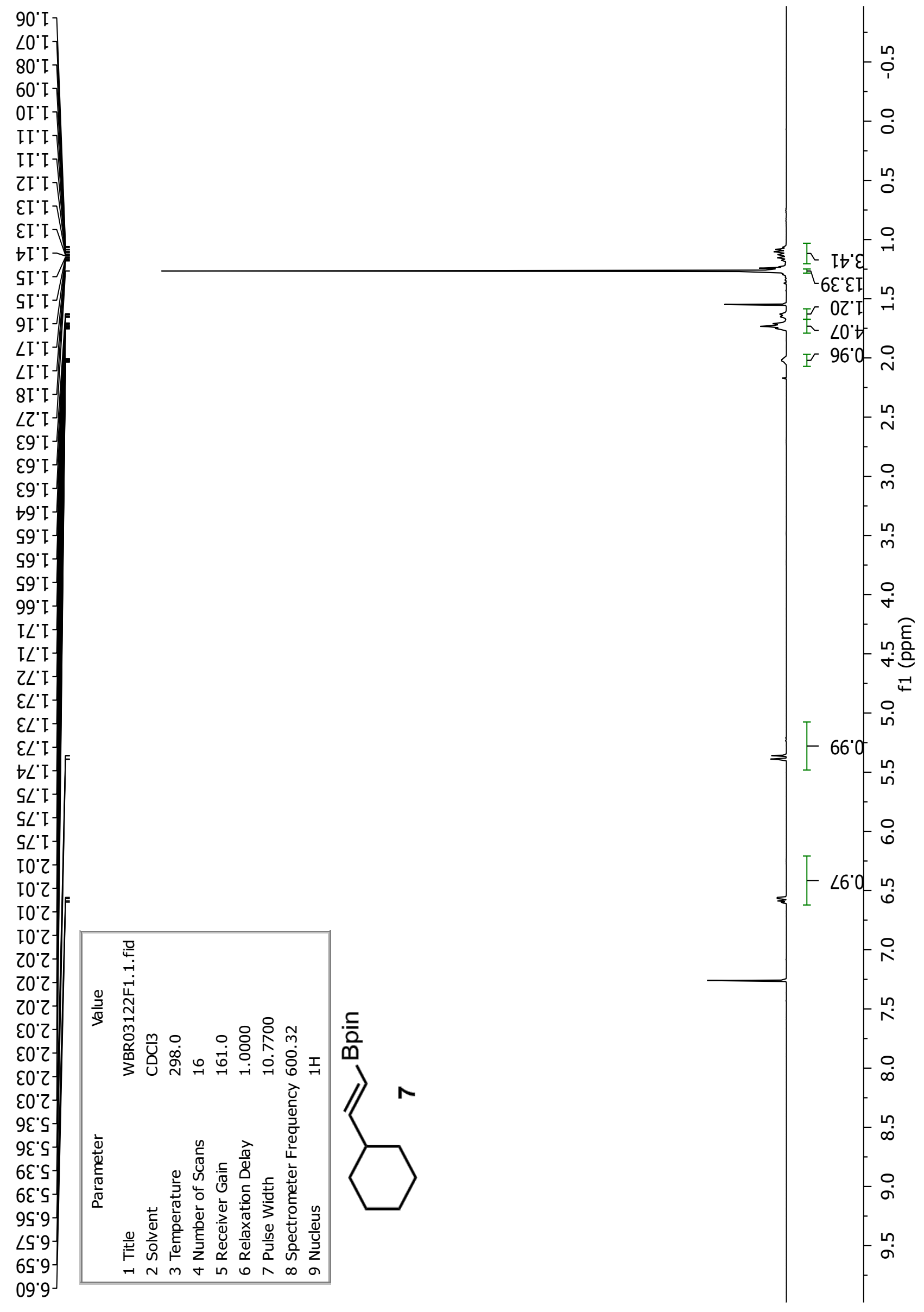




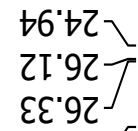

$80^{\circ} \mathrm{Z} \varepsilon^{\jmath}$

$0 t^{\circ} \varepsilon t-$

हI'ย8-

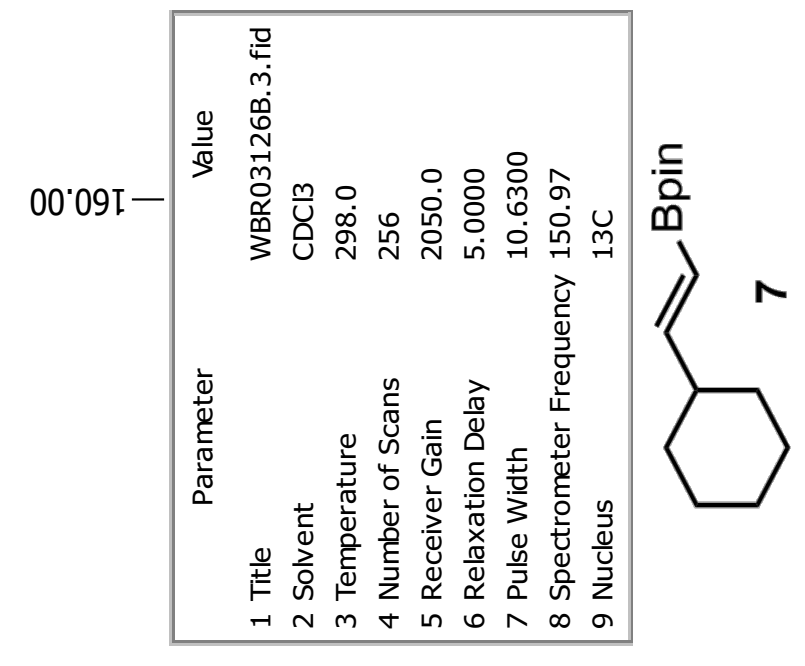

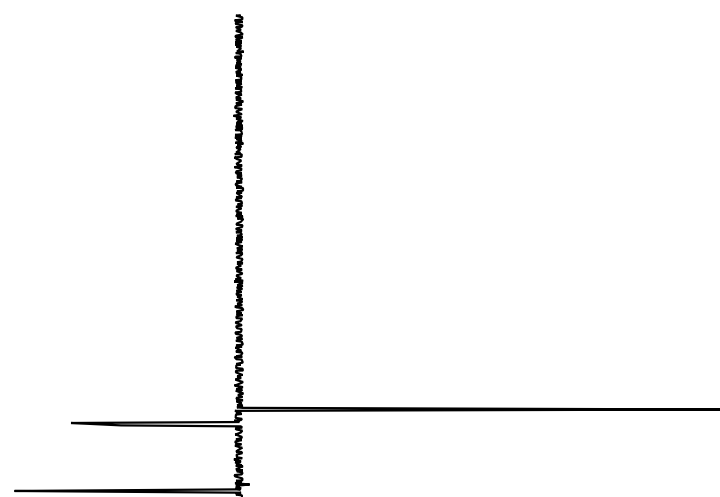

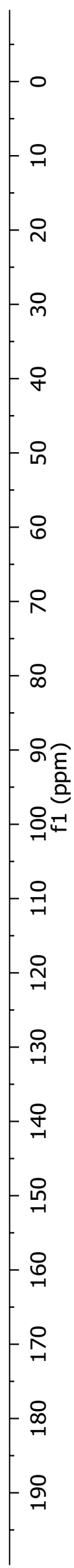




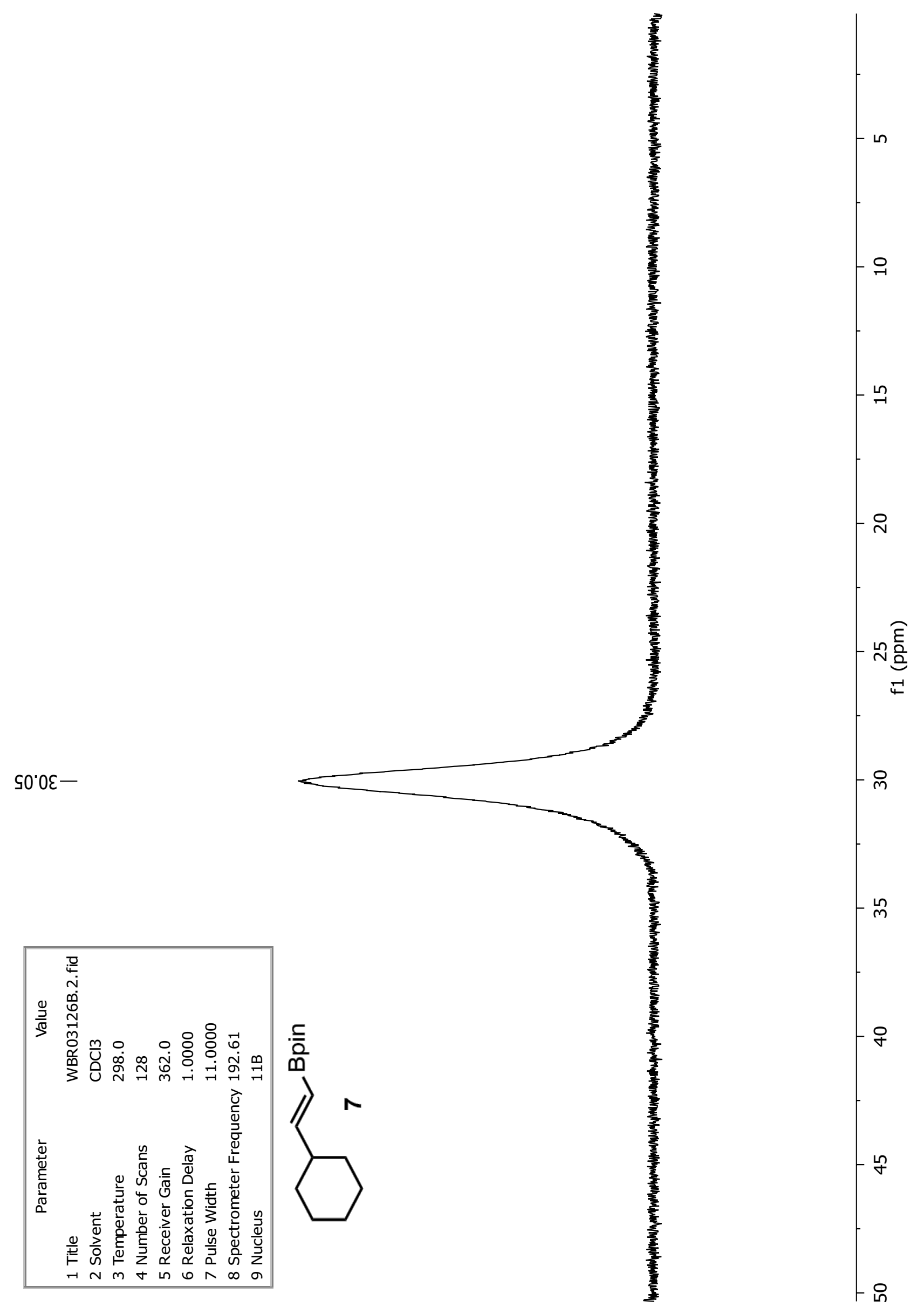




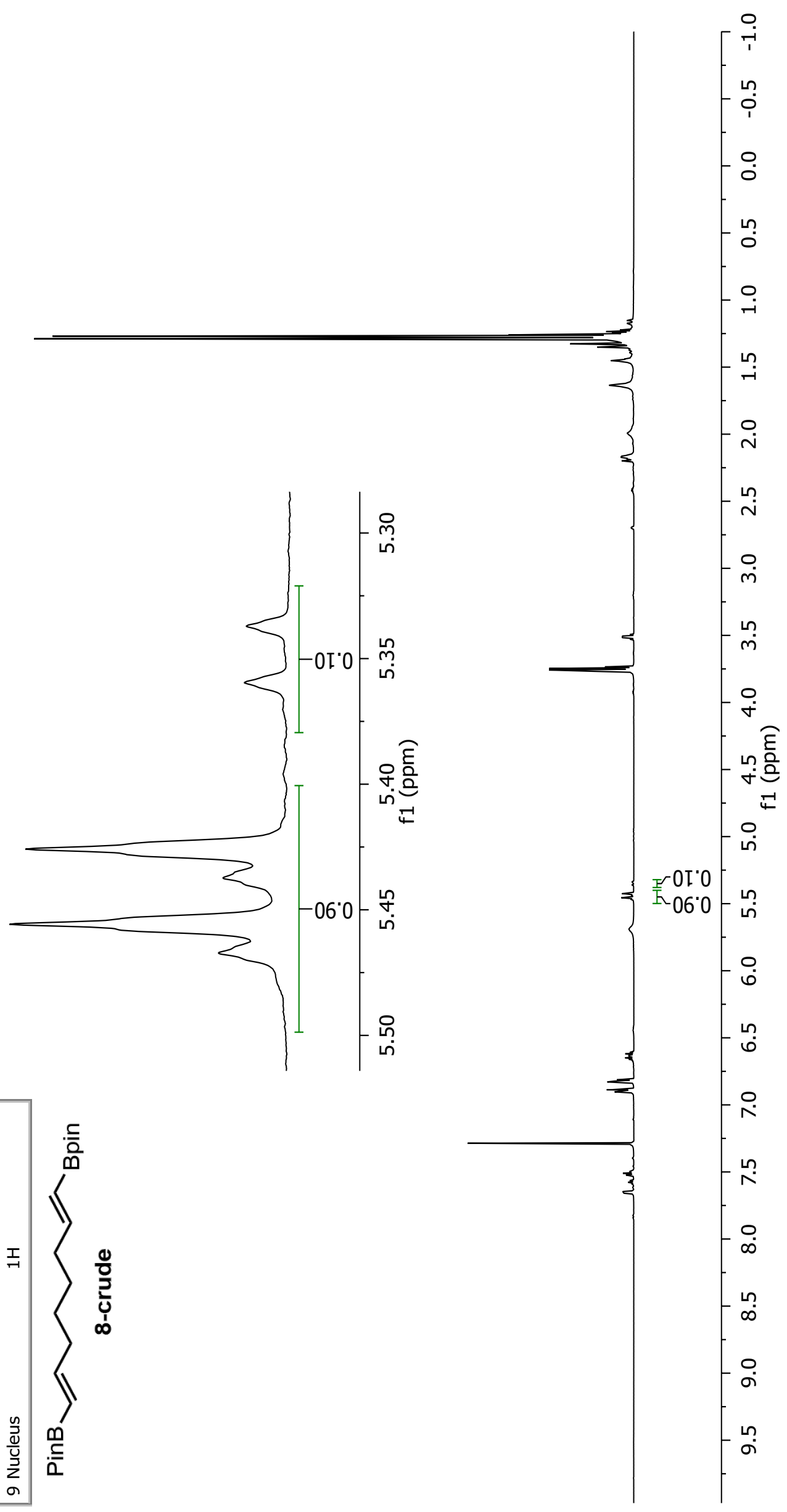




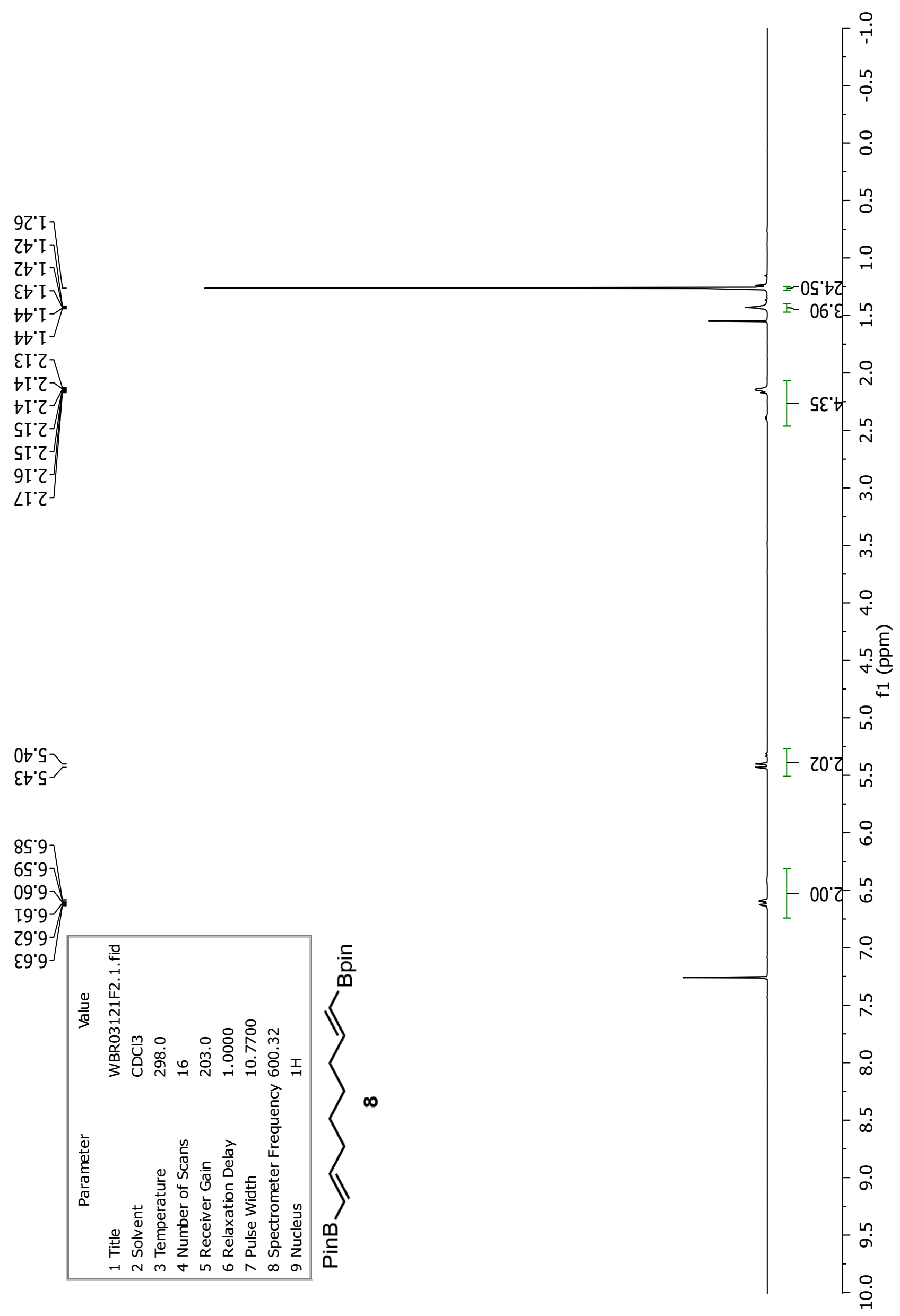




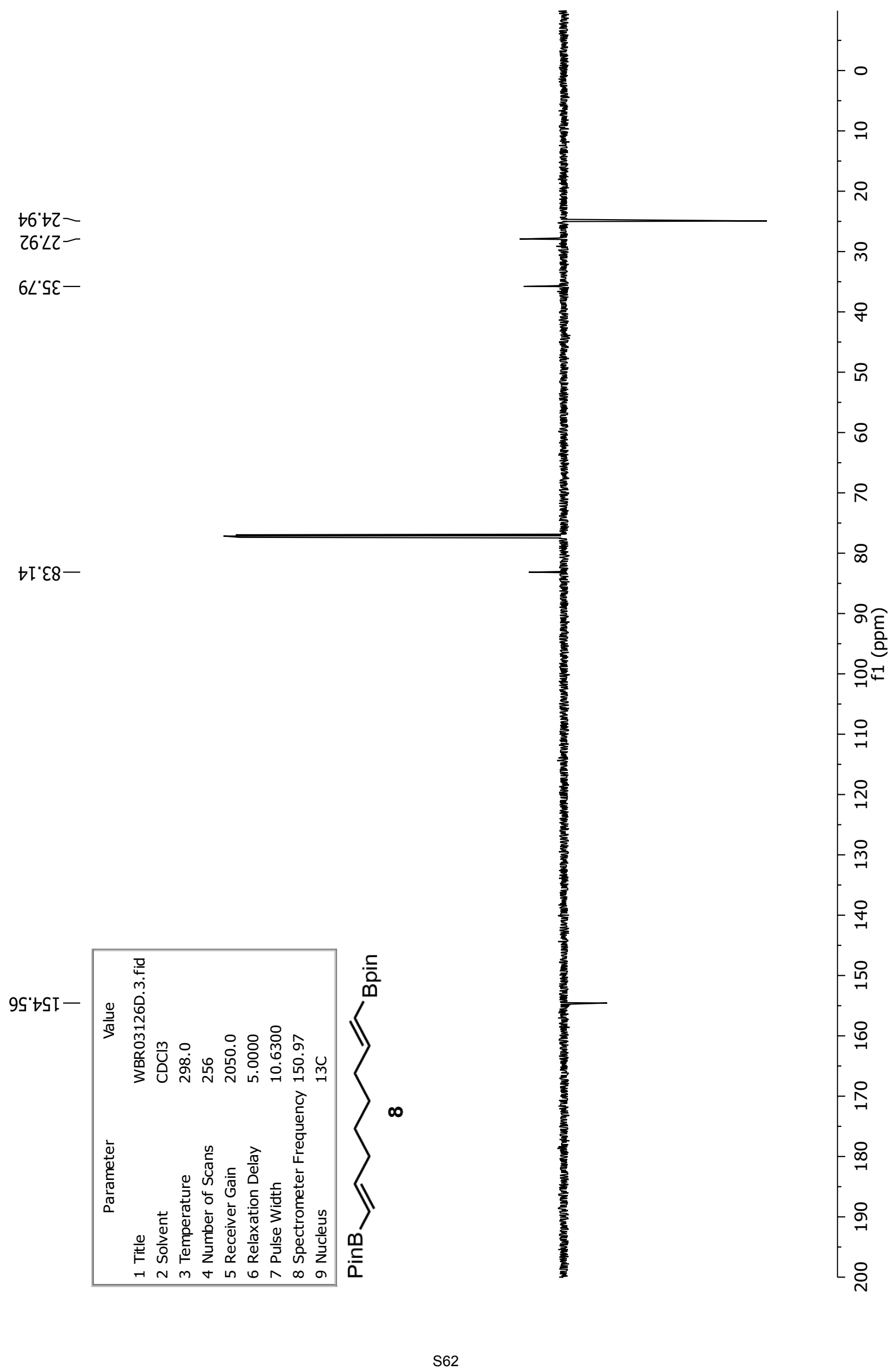




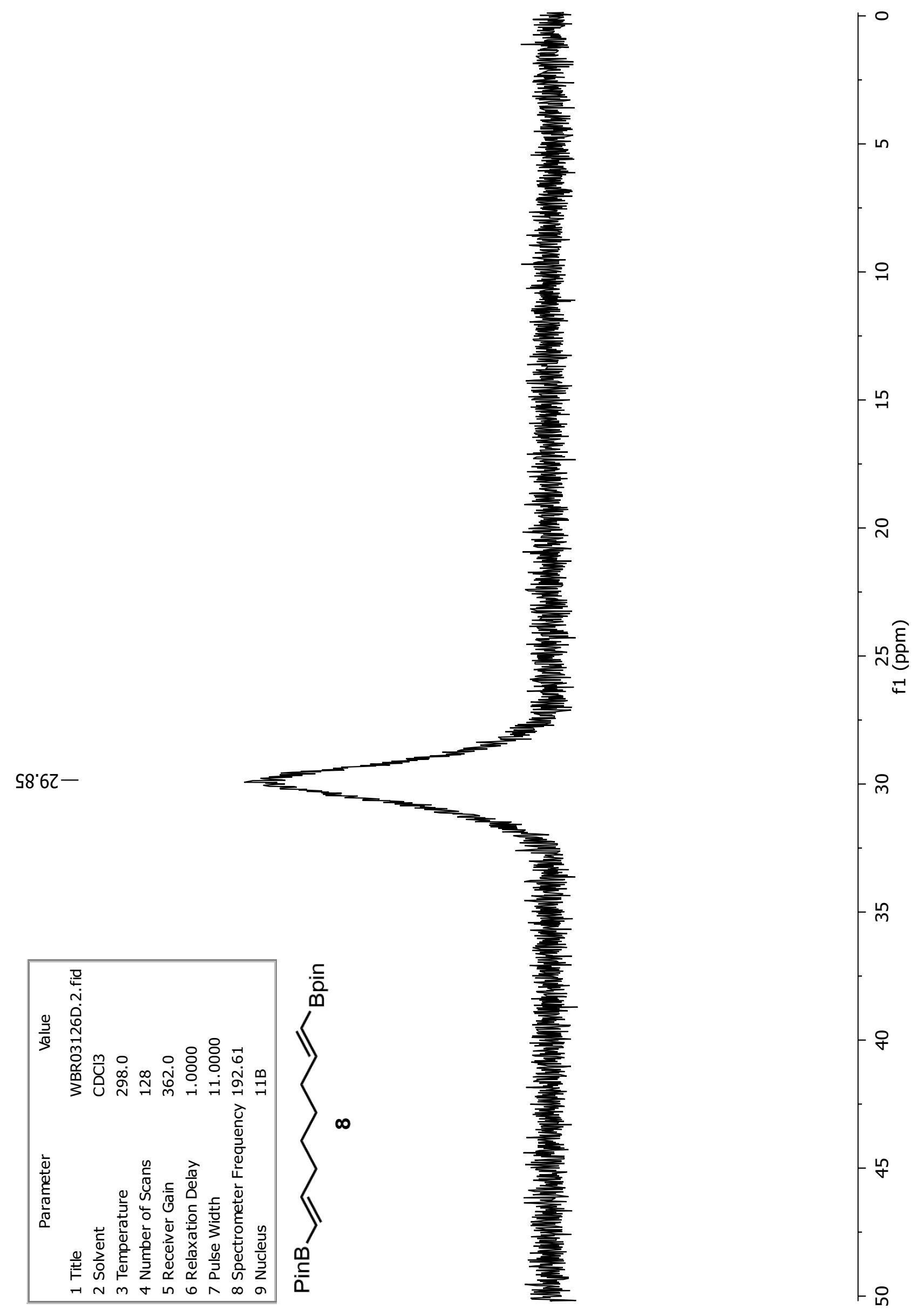




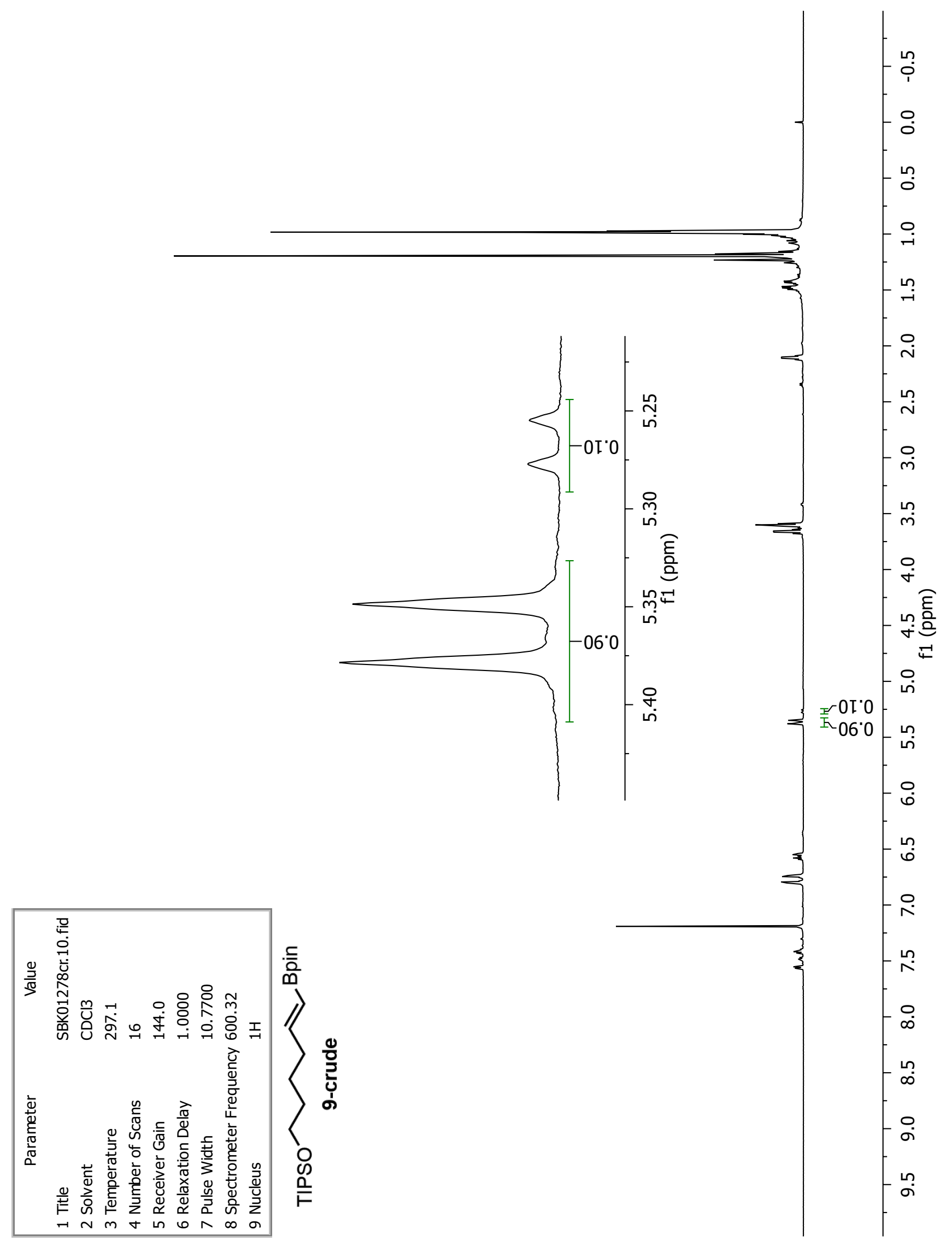




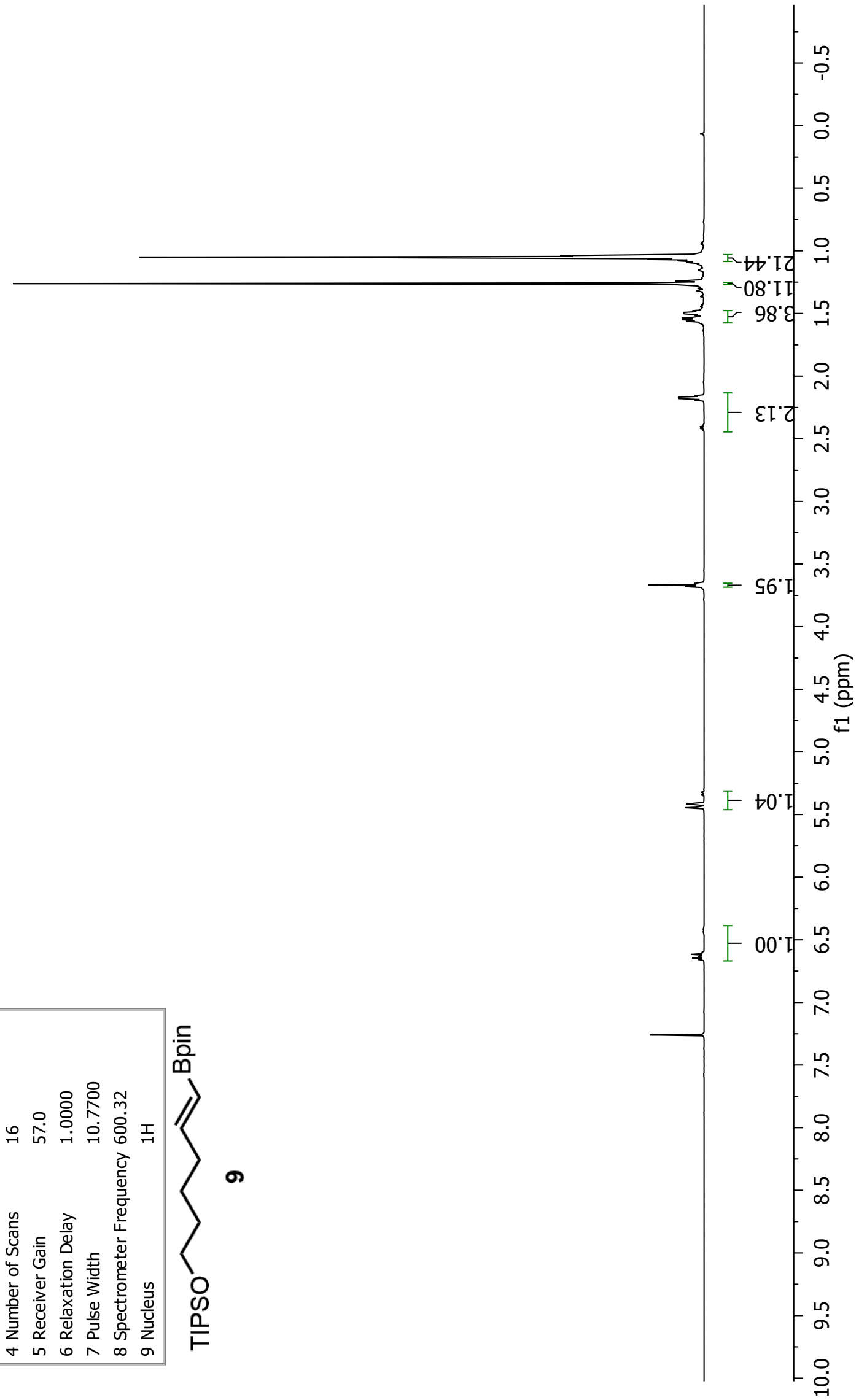

ZE'S
$\left.\succ \varepsilon^{\prime} S\right]$

Zt: $\mathrm{s} \cdot \mathrm{s}]$

$0 t^{\circ} 9$

โt 9

$\varepsilon t^{\prime} 9$

tb. 9

St. 9

โ9.9

乙9.9

$\varepsilon 9^{\circ} 9$

†9 9 -

ธ9.9-

문

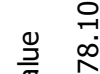

$\stackrel{5}{\infty}$

99.9

$\frac{\pi}{3} \stackrel{5}{3}$

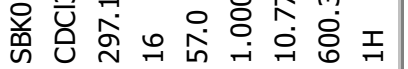

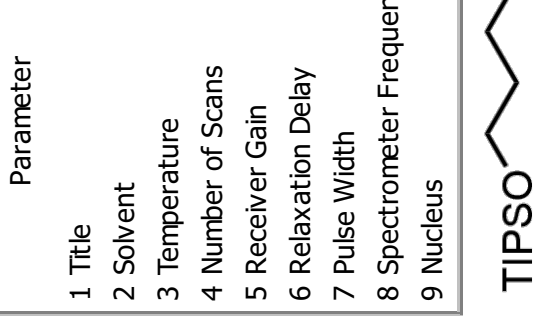


$\angle I ' Z I-$

6I' $8 \mathrm{I}$

$\angle 9^{\circ} \circ Z$
$\forall 6^{\circ} \circ Z$

69'حع-

$\angle L ' \varsigma \varepsilon-$

દદ'๕9-

$\downarrow I^{\prime} \varepsilon 8-$

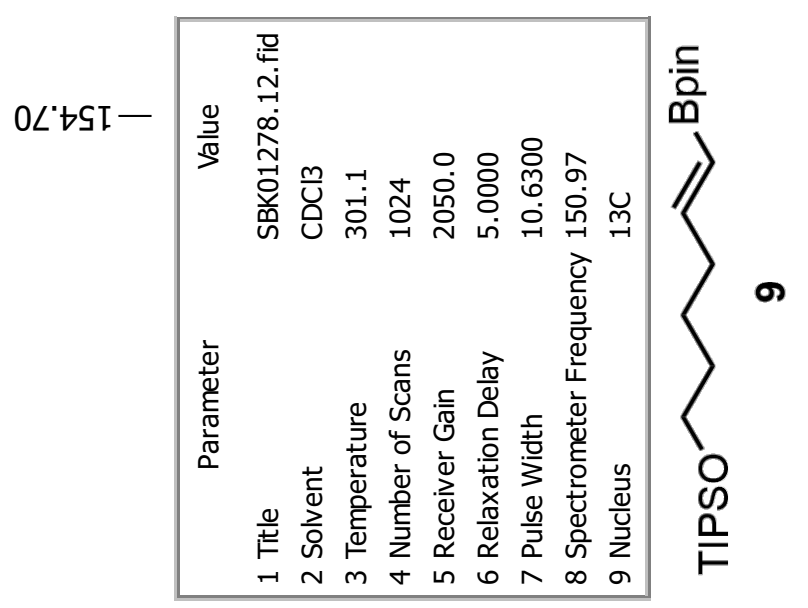

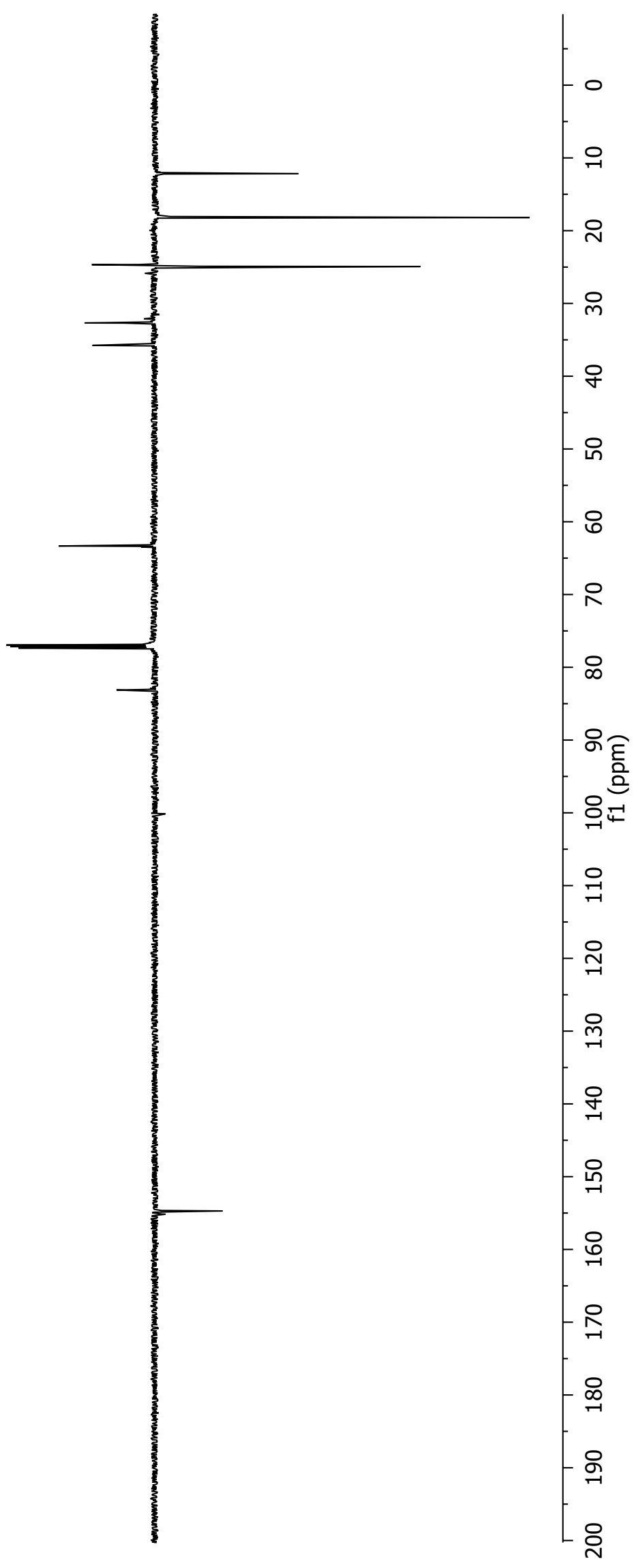




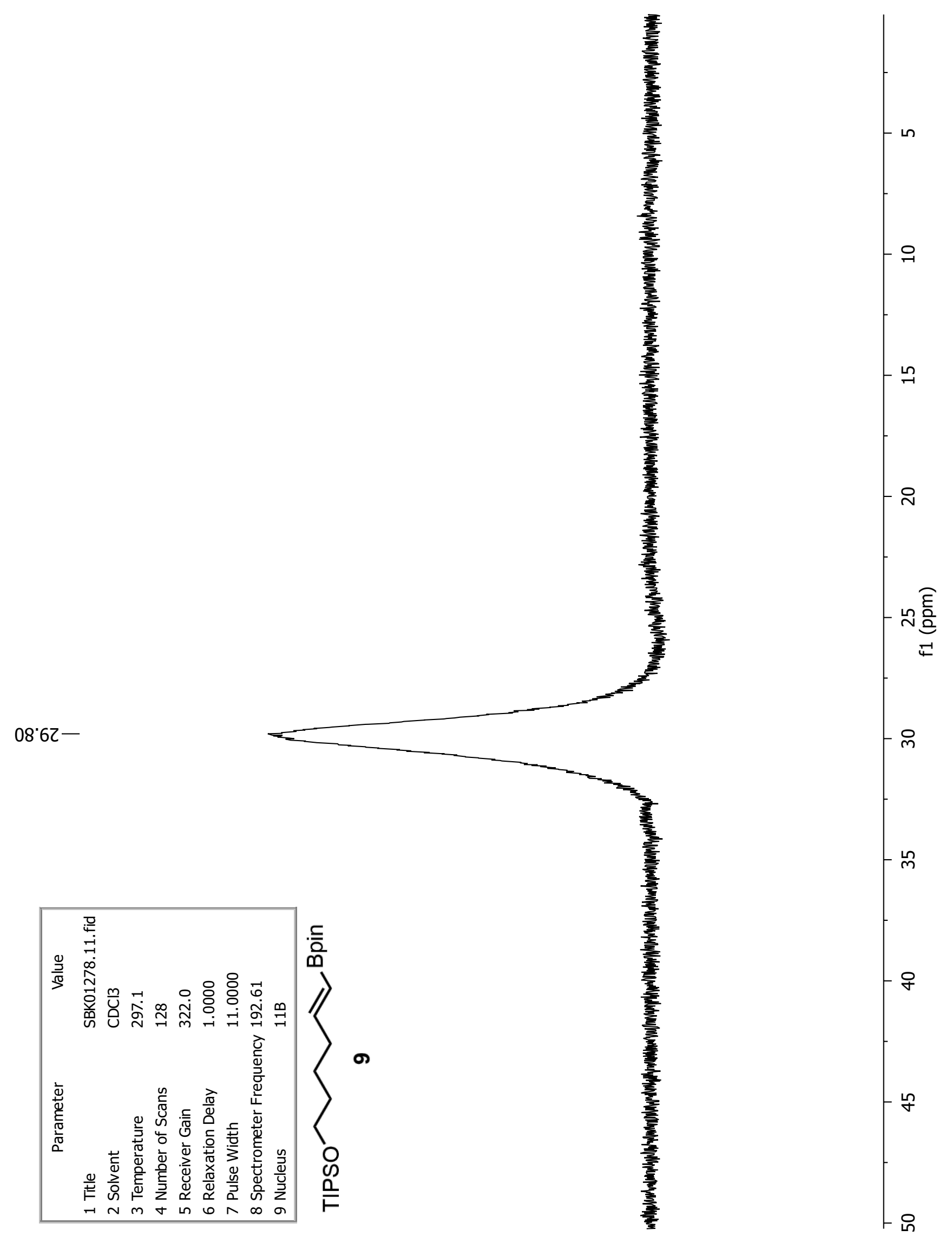




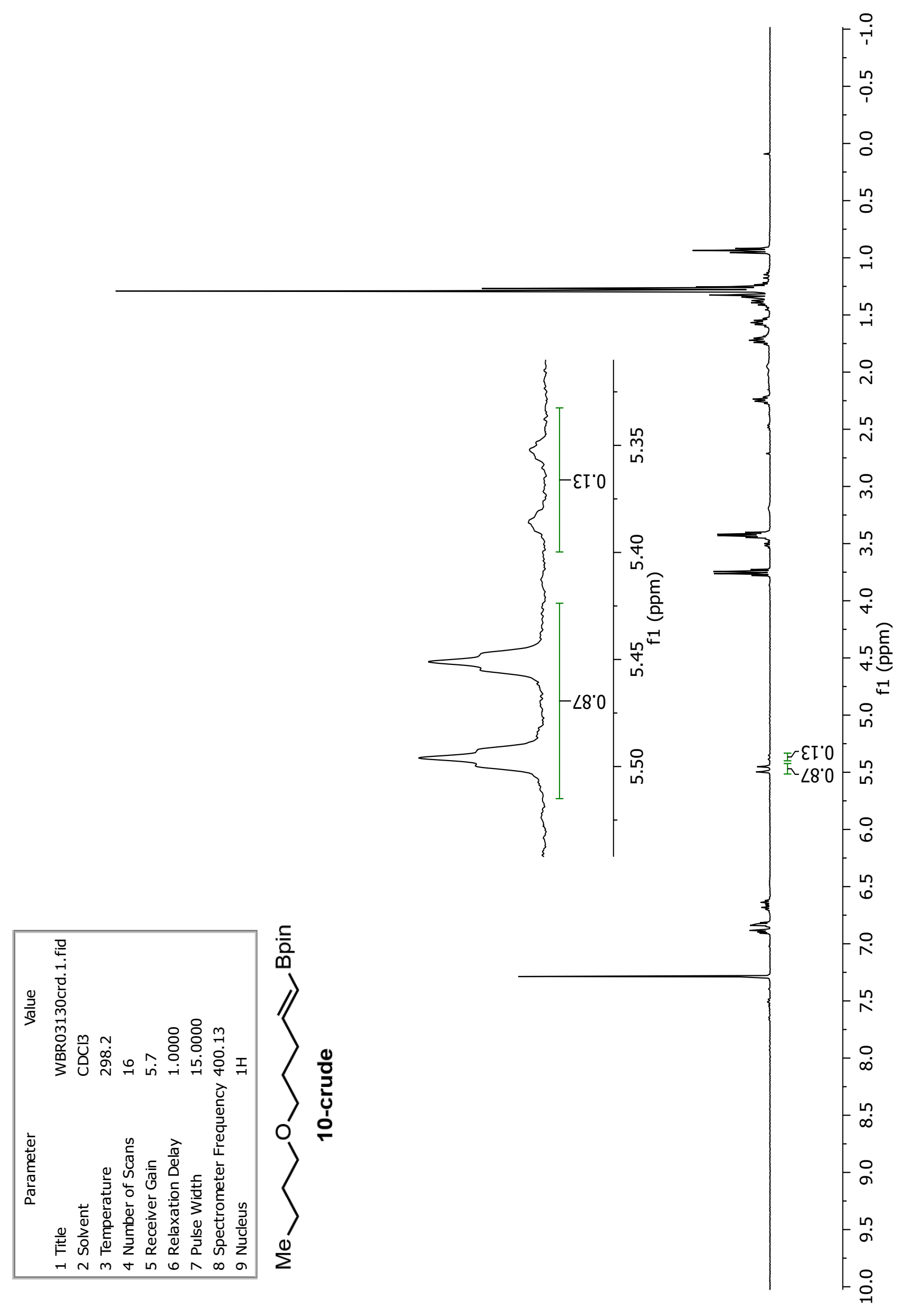




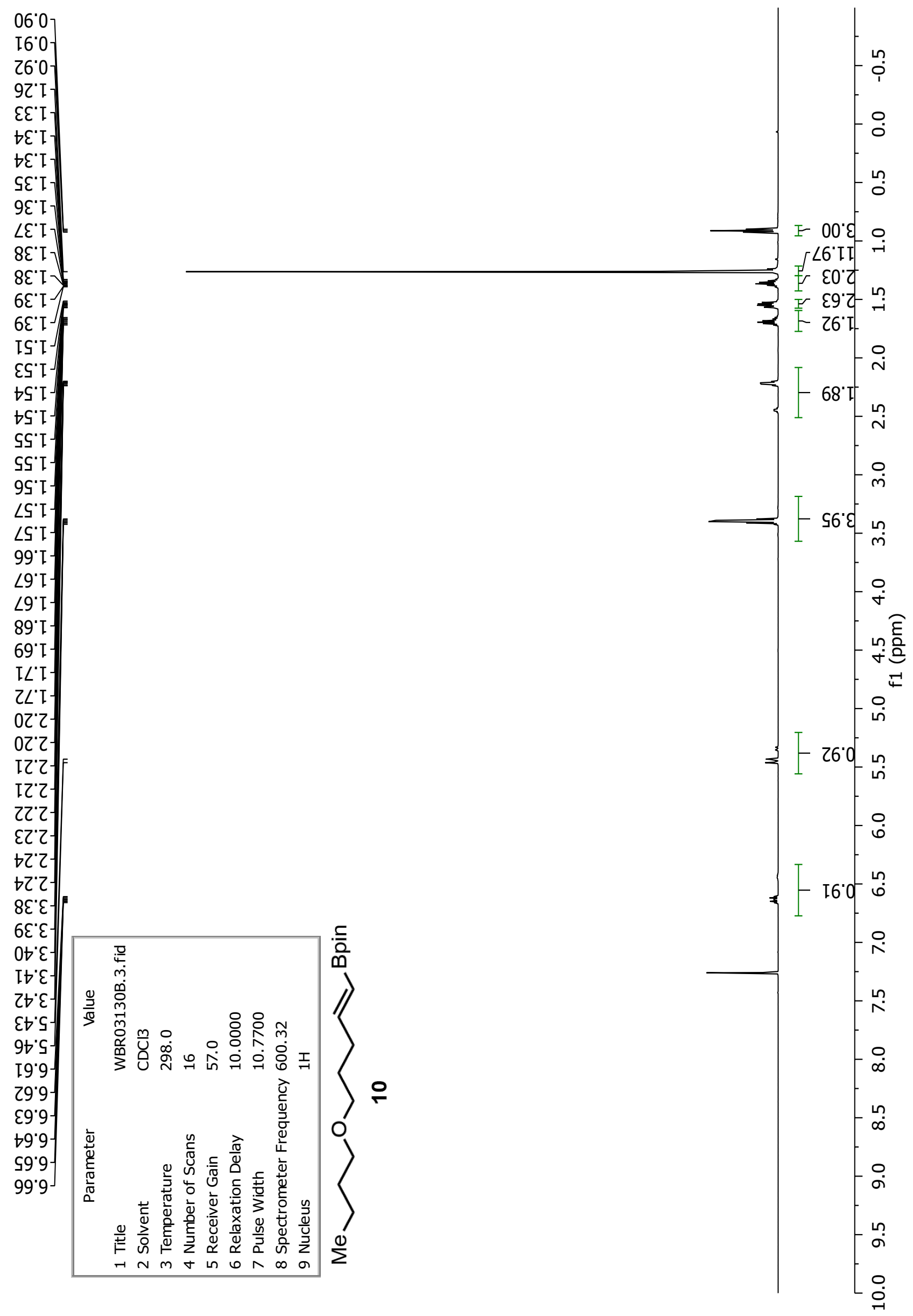



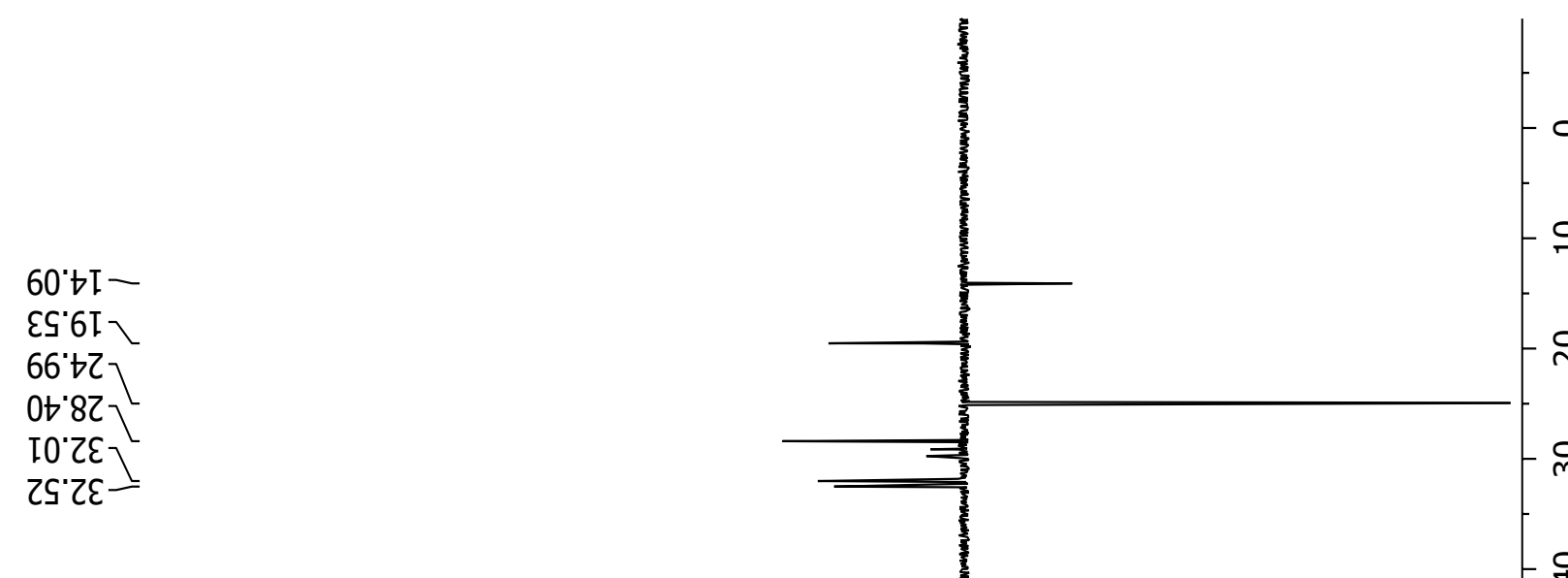

$6 Z^{\circ} 0<$
$08^{\circ} 0 L^{\prime}$

LI'E8-

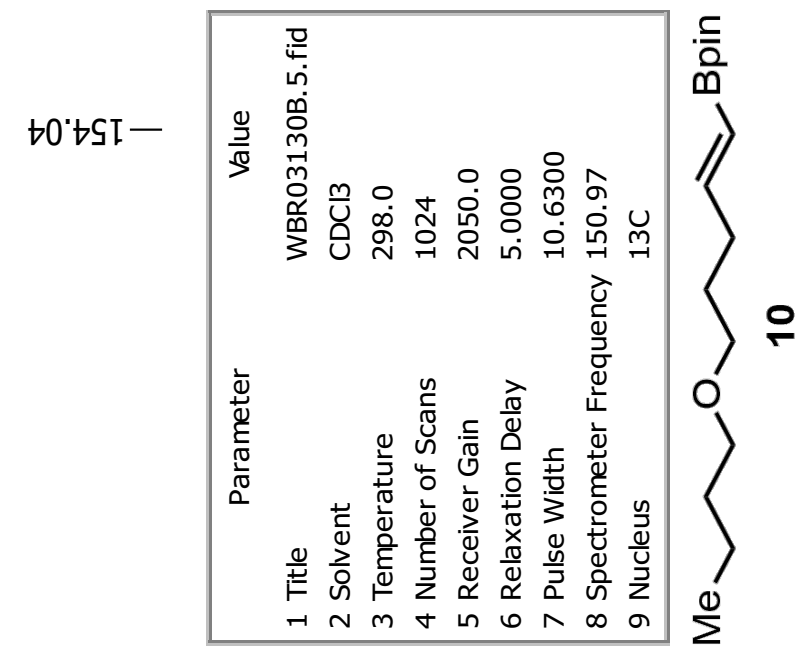




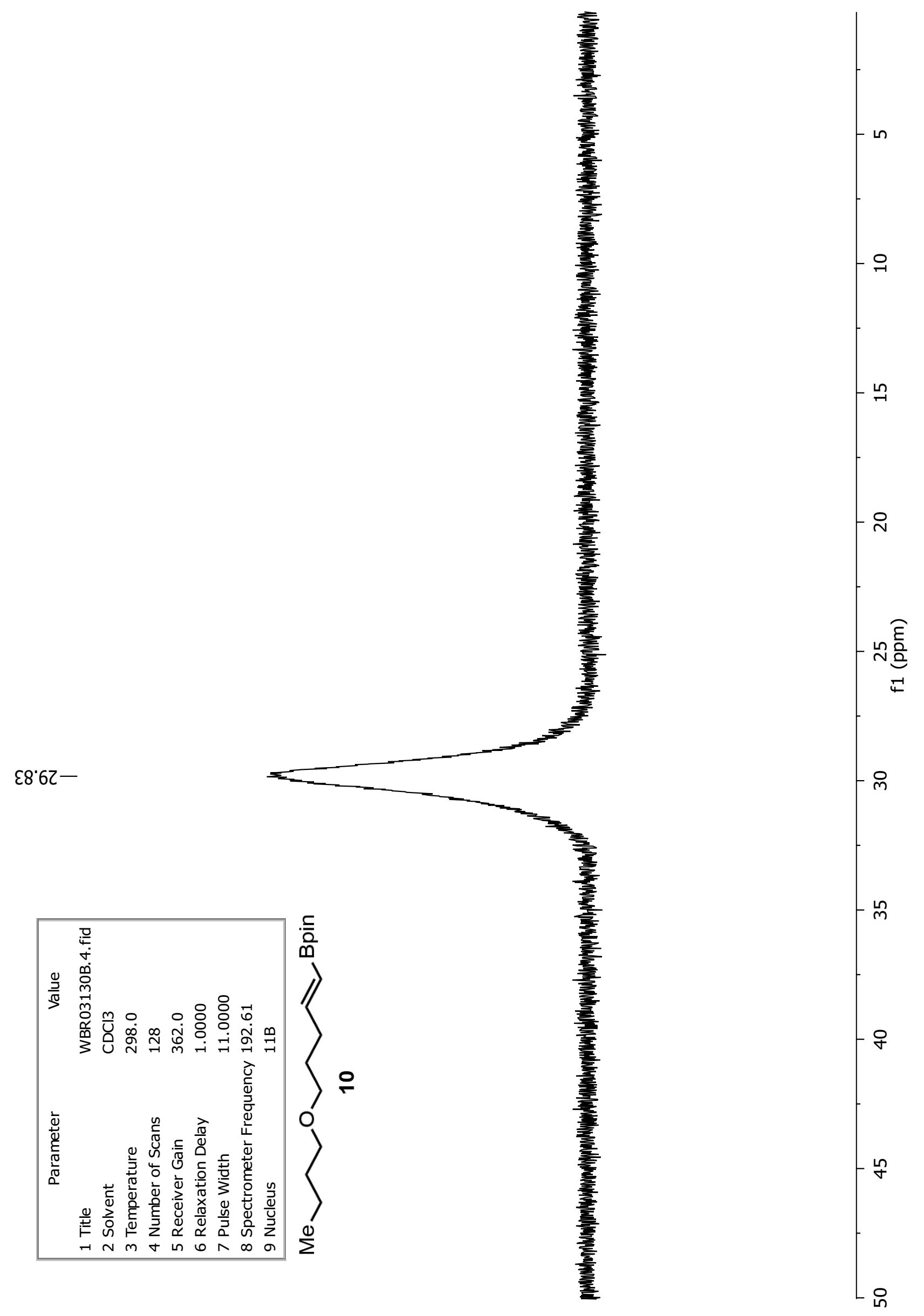




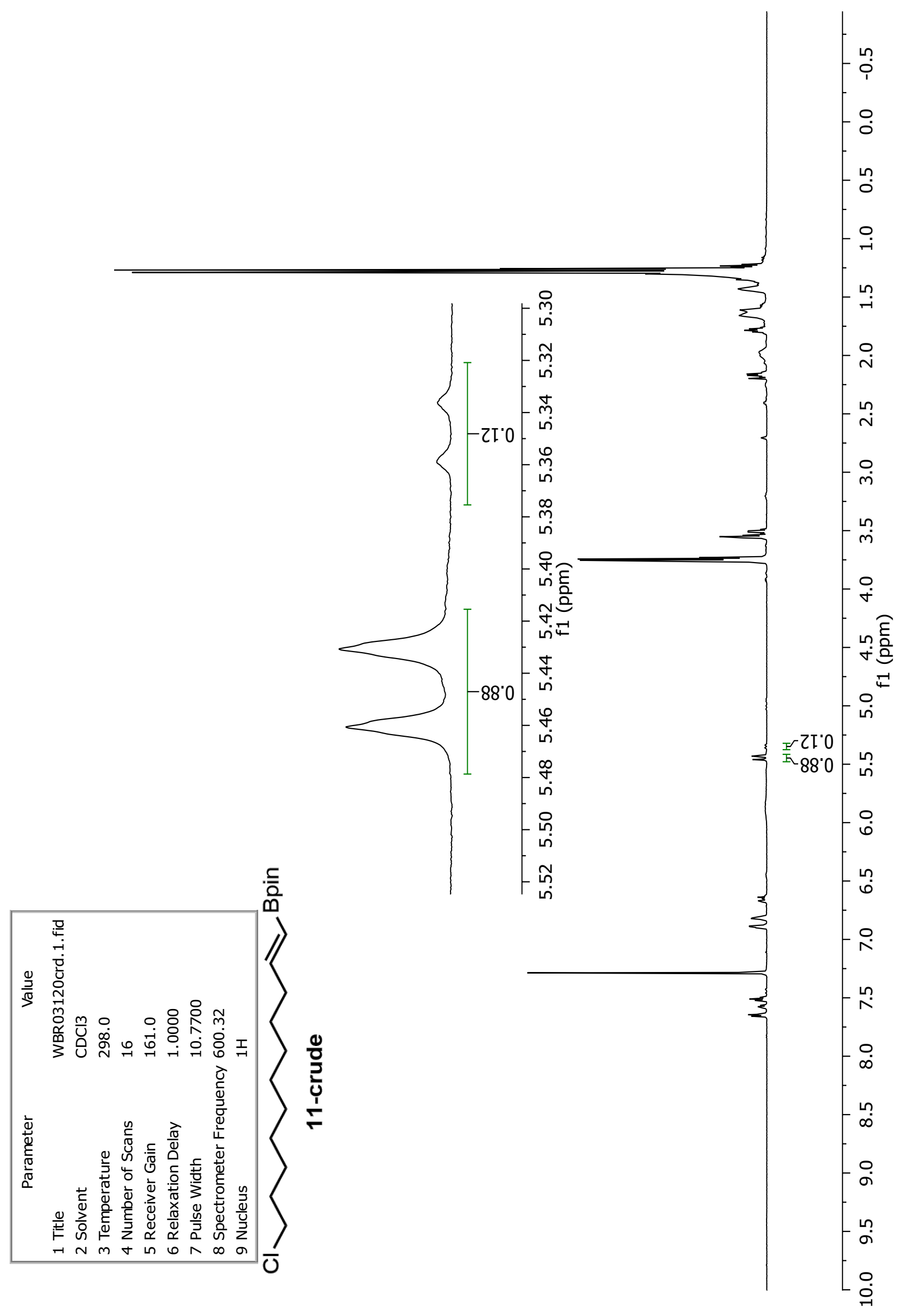




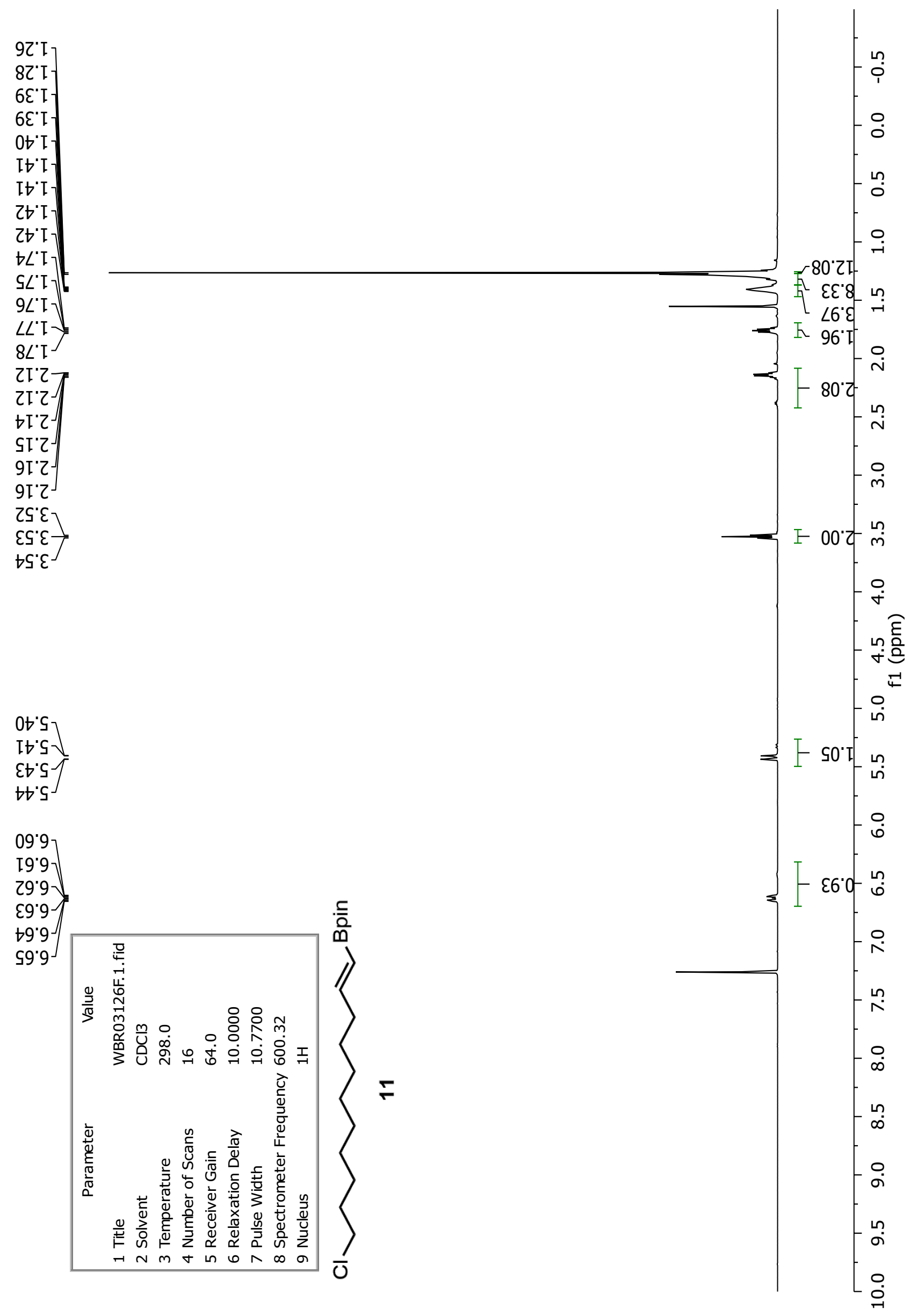



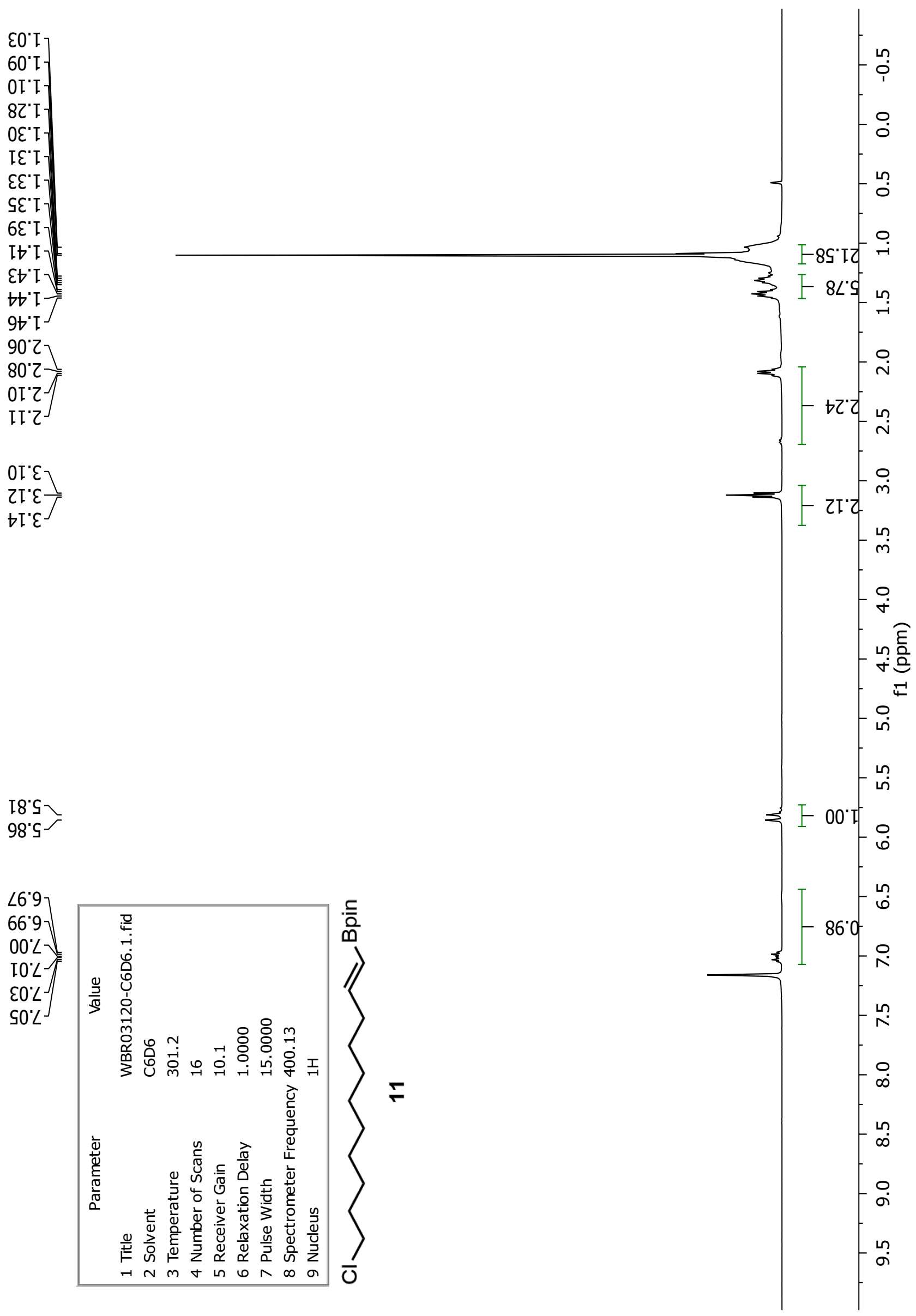

I8' 1

98. 


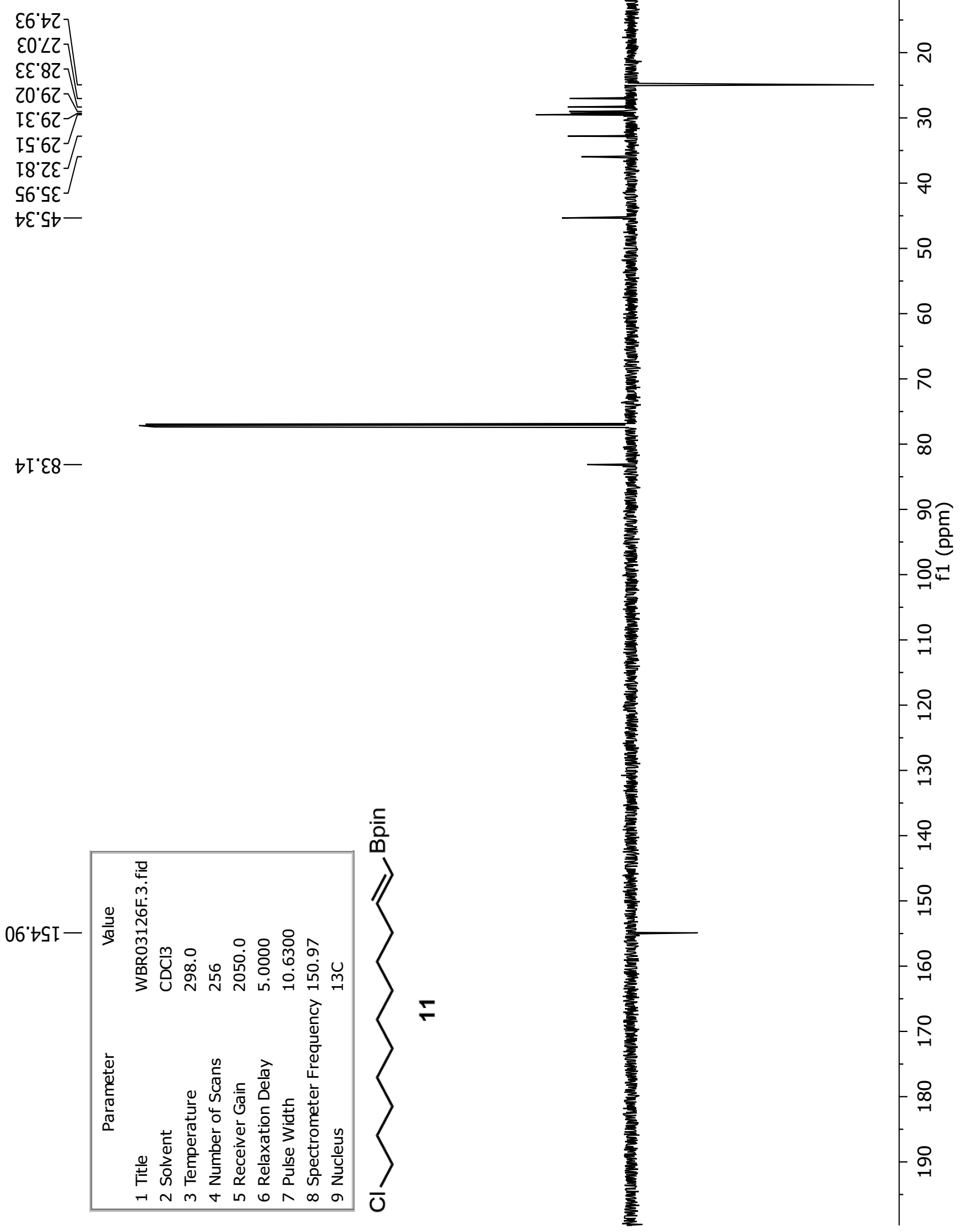




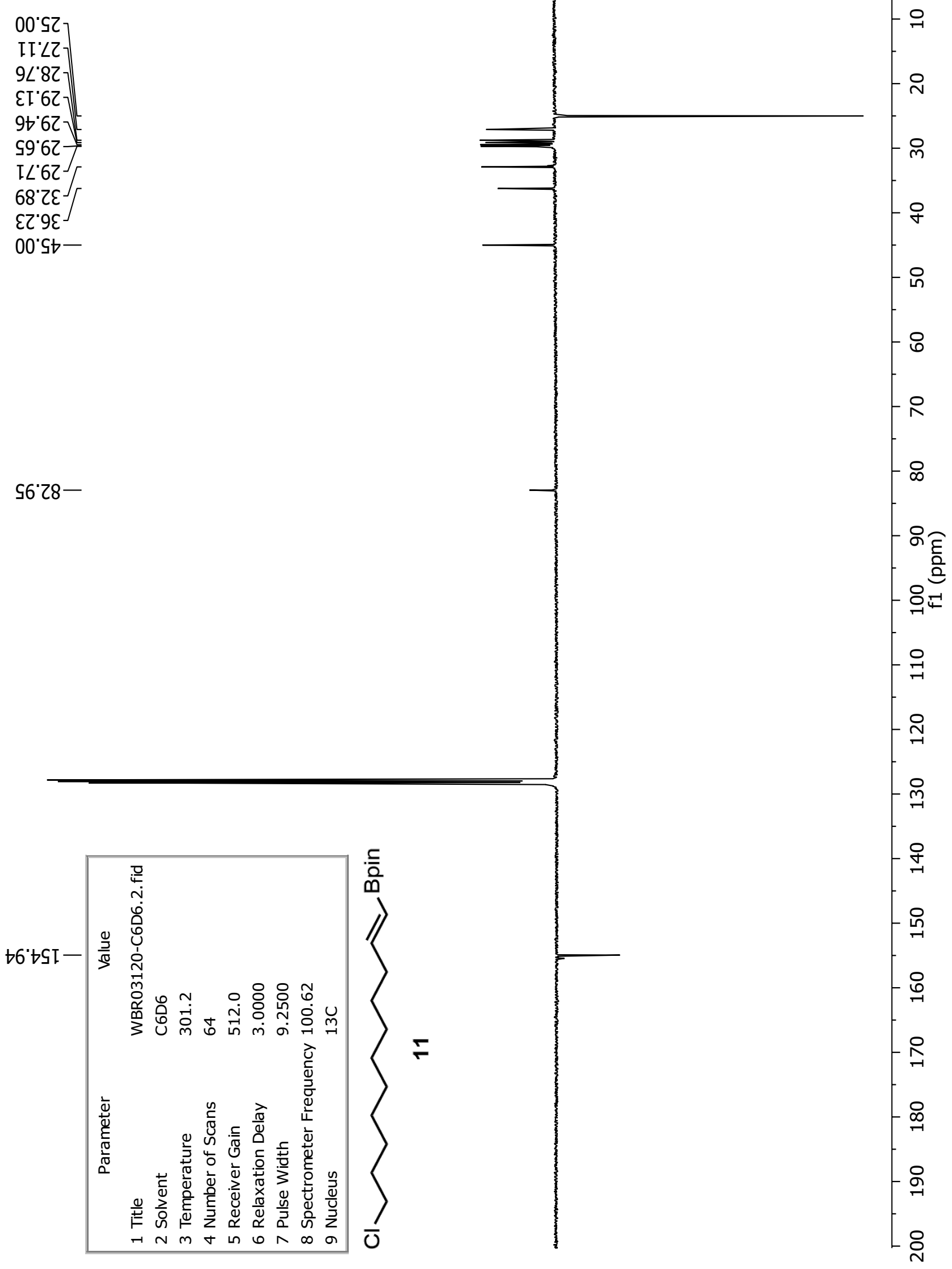




$$
\frac{1}{1}
$$




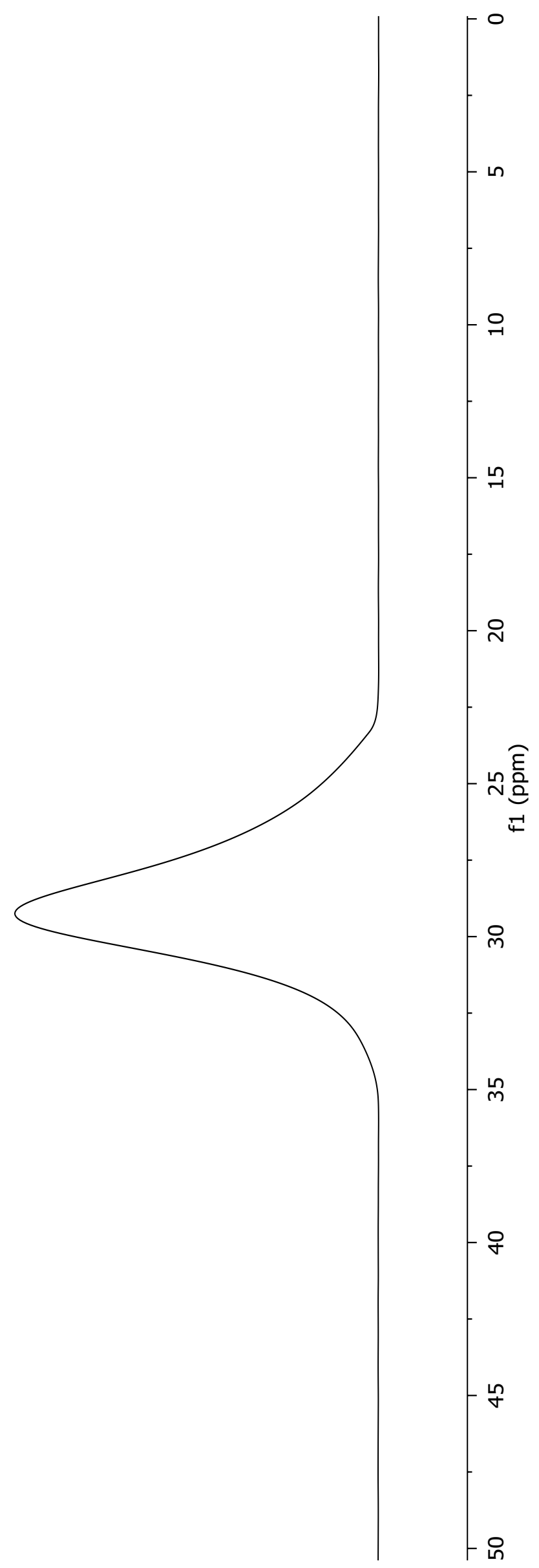




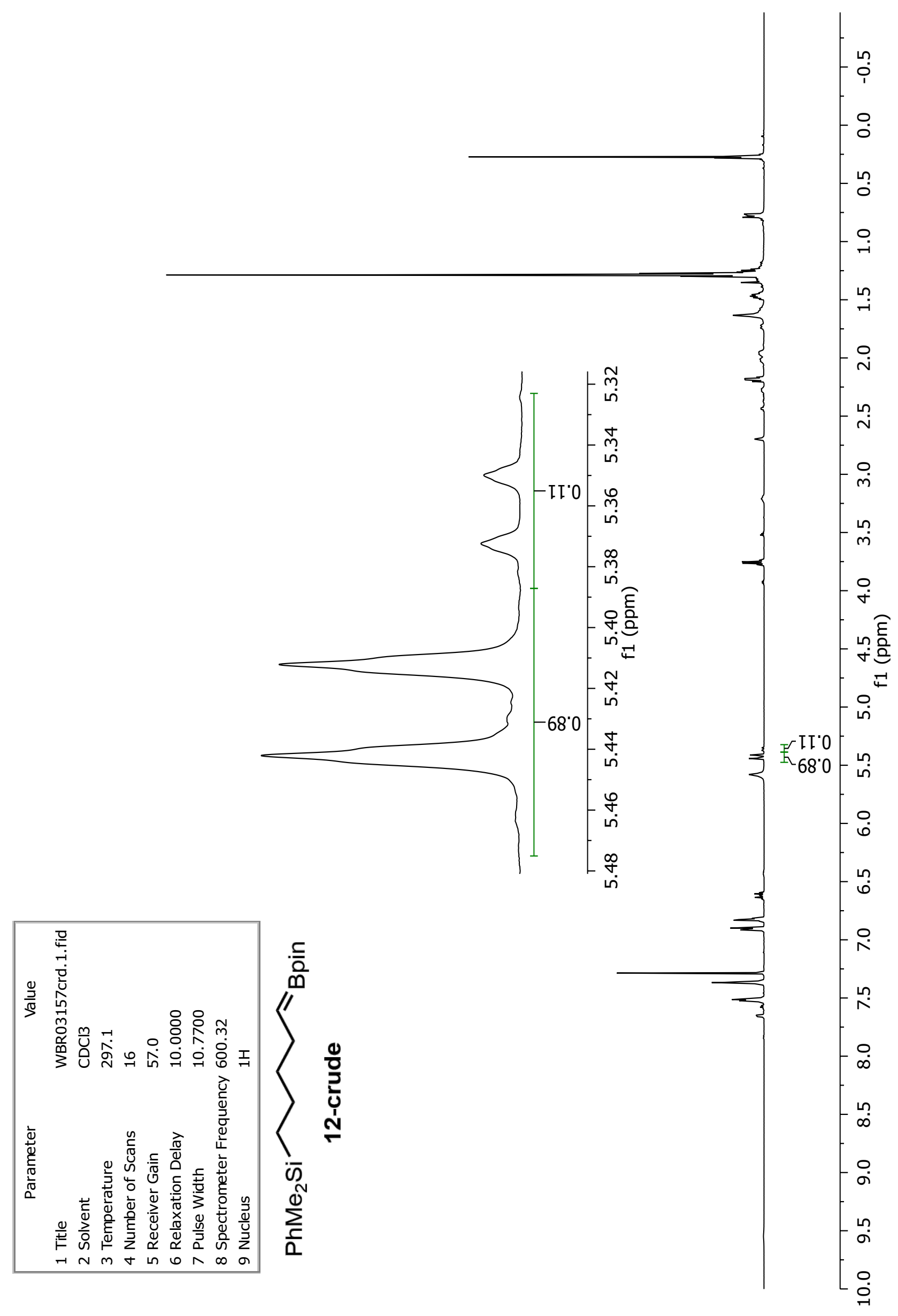




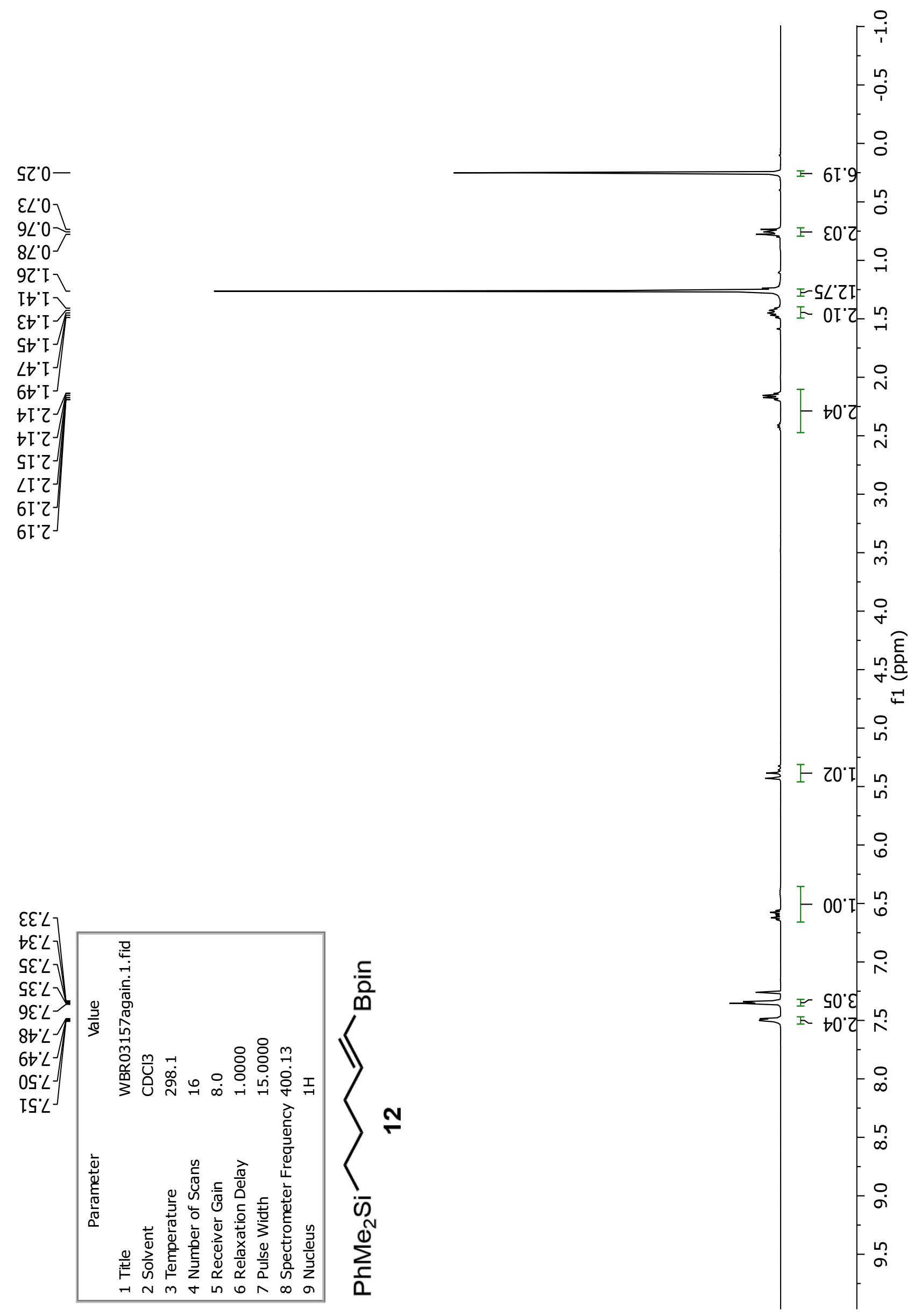


$56^{\circ} 2-$

เ9'SI-

$\angle 8^{\circ} 22$

$26^{\circ}+22$

โ8.6ย-

عI'ย8-

†8:LZI-

89. ¿દโ

ZS.6EI-

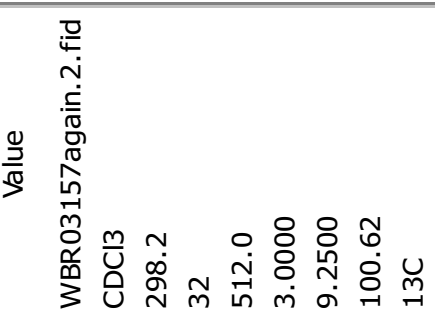

든

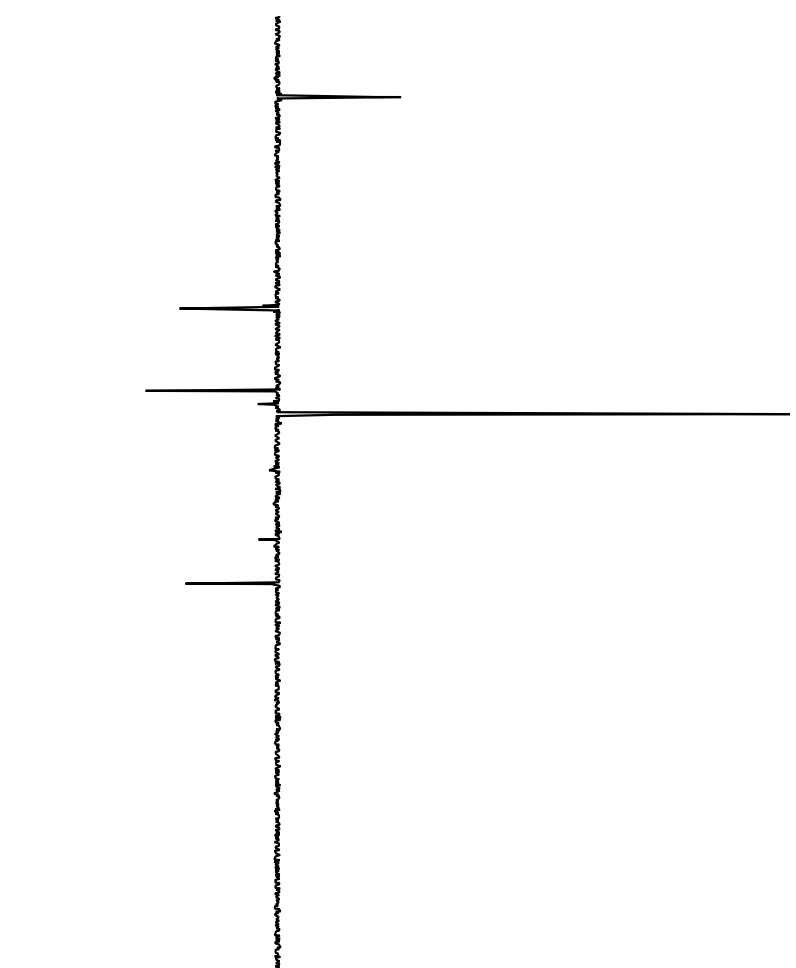

우

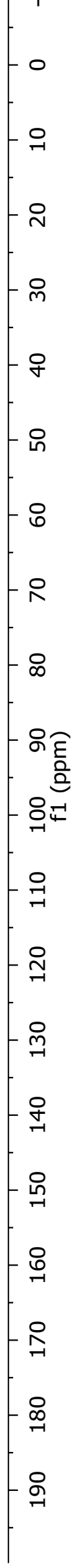

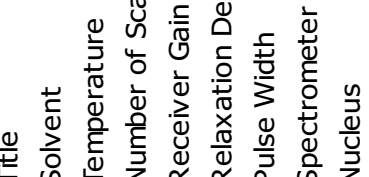

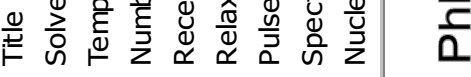

$\sum N$

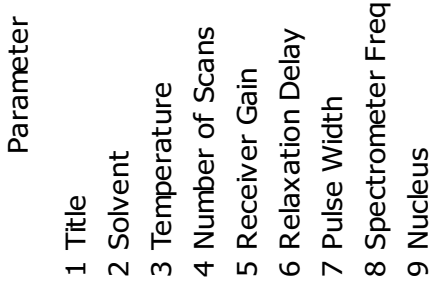




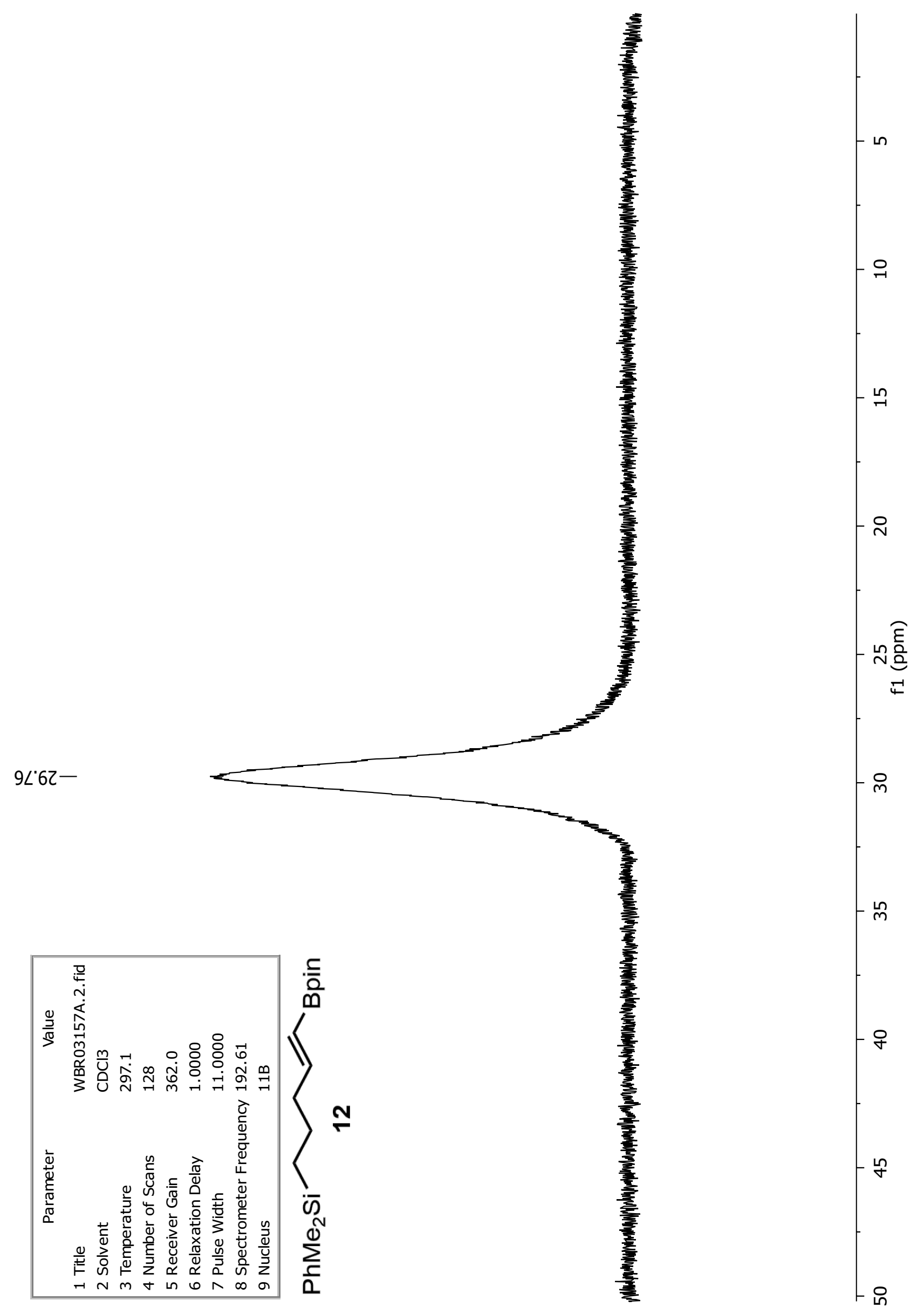




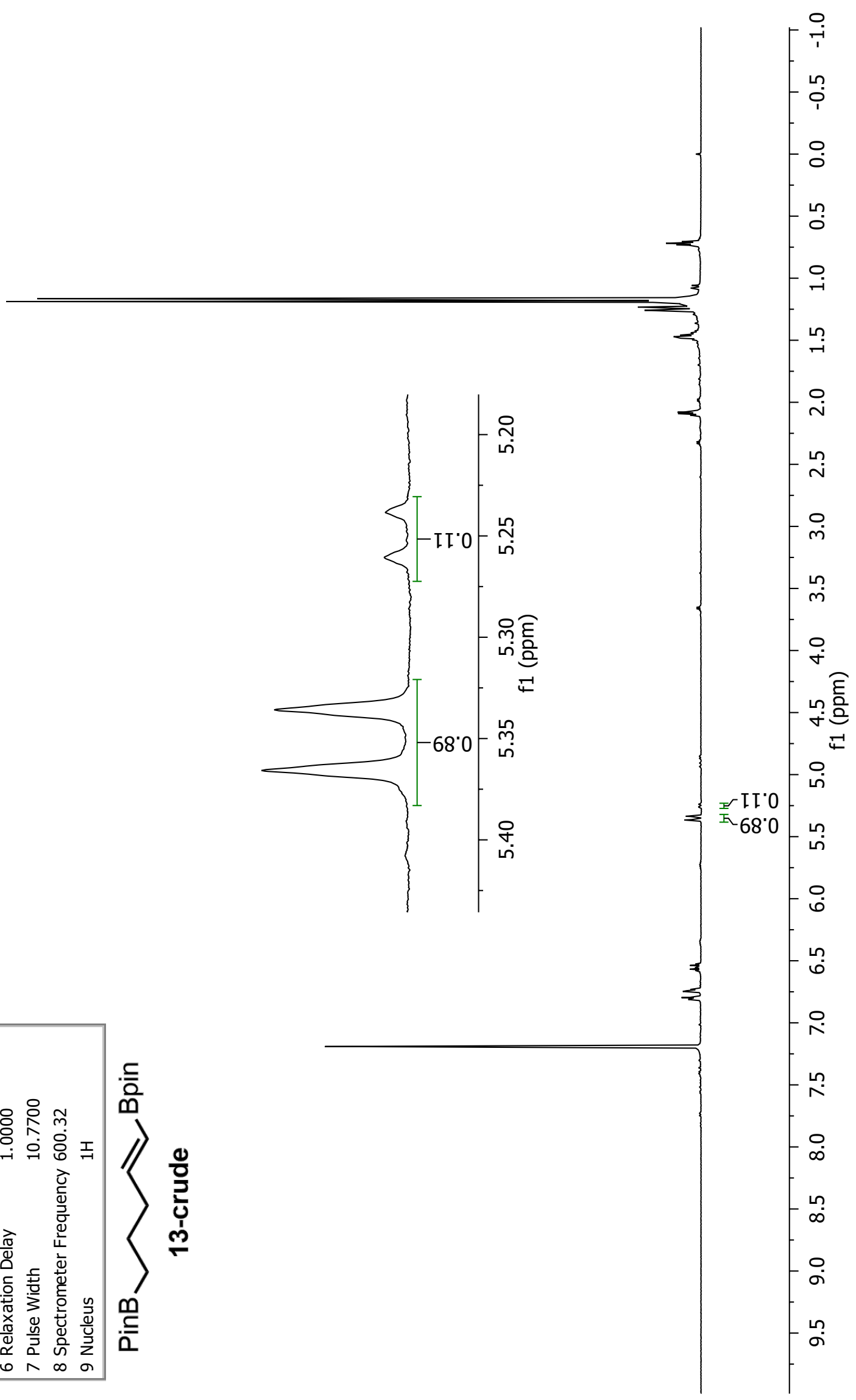


$\checkmark \angle{ }^{\circ} 0$

9L'0]

$8 L^{\circ} 0$

IZ' ' ]

$\varepsilon \mathrm{C}^{\prime}[]$

$\angle b^{\prime}[]$

6t']

IS' $I \sqrt{ }$

ES' [

OI' 2

ZI'Z-

DI' 2

SI'ح

$\angle Z^{\prime} S$
IE'S

$\angle \varepsilon^{\prime} S$

$\angle \varepsilon^{\prime} S$

$\left.\angle E^{\prime} S\right]$

It'S

It: 5

ऽ.'9

$8 \varepsilon^{\prime} 9$

$6 \varepsilon^{\circ} 9$

โ 5

$\varepsilon t 9$

95. 9

$\angle S^{\circ} 9$

65.9

$09^{\circ} 9$ -

Z9.9-

$\frac{5}{5}$

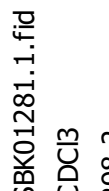

ع.9 9

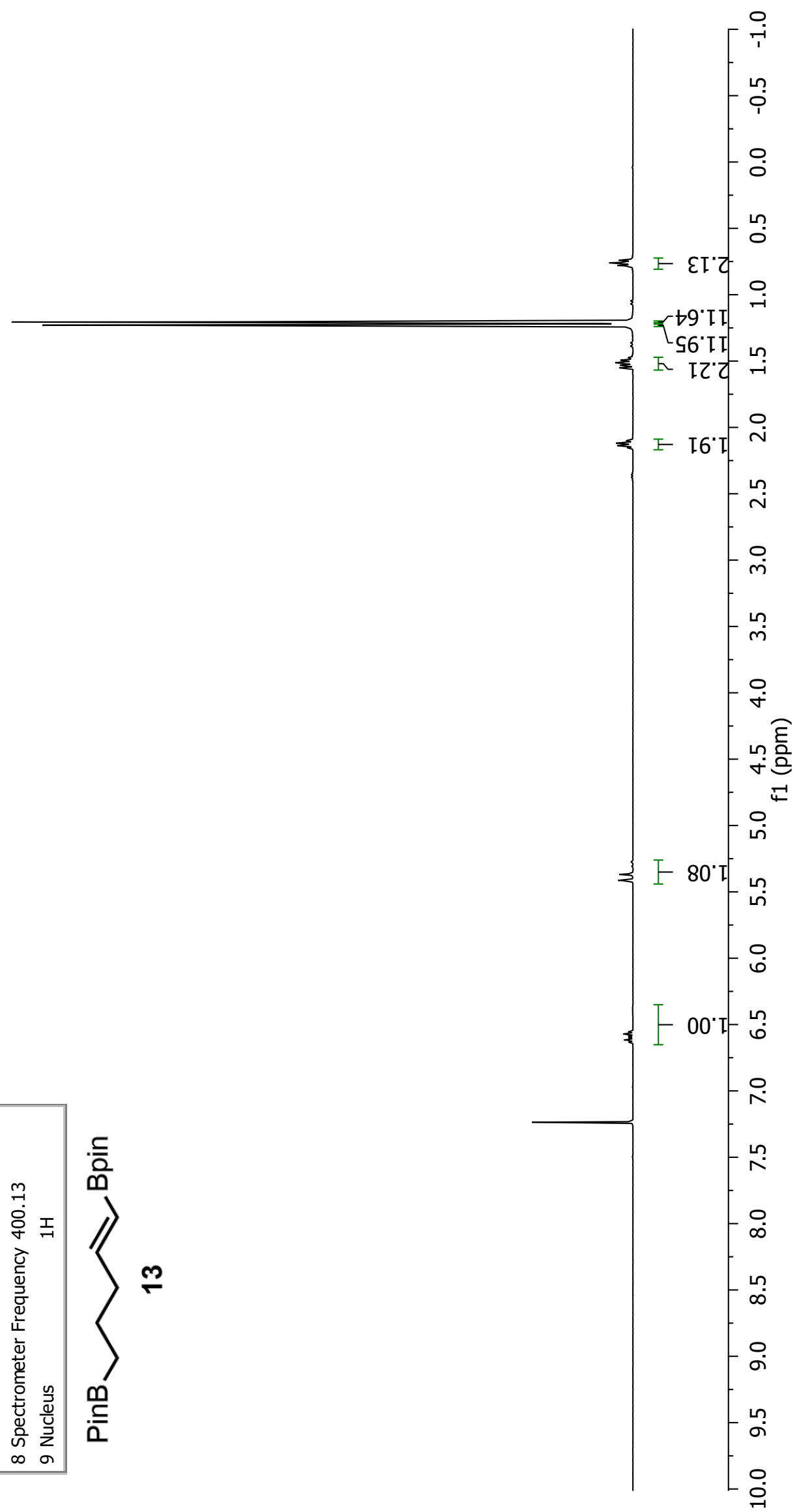



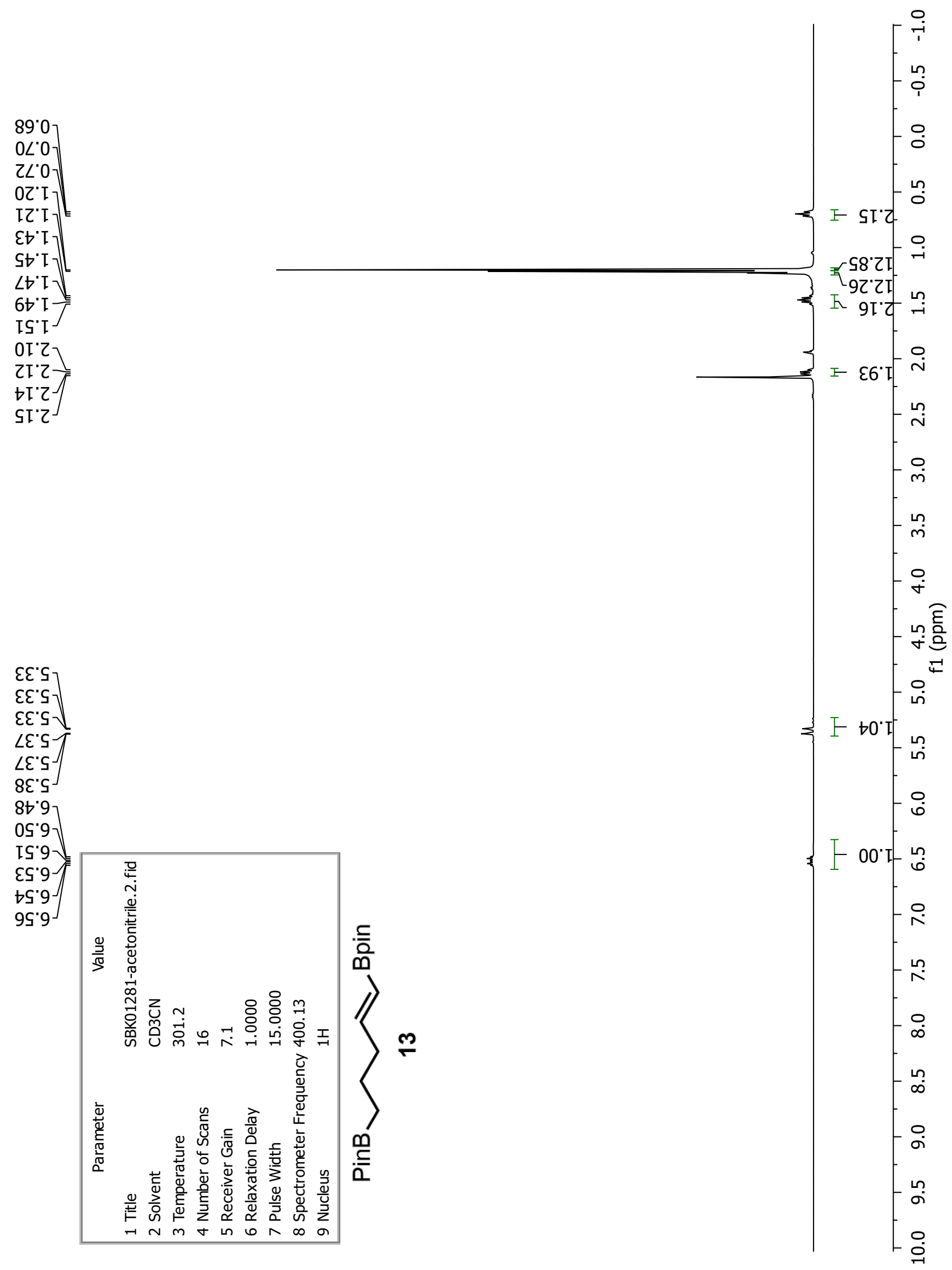
$\varepsilon 6^{\circ} 2 Z-$

$96^{\circ}$ เて二

$0 S^{\circ} 8 \varepsilon-$

†० $\varepsilon 8$

$80^{\circ} \varepsilon 8^{\prime}$
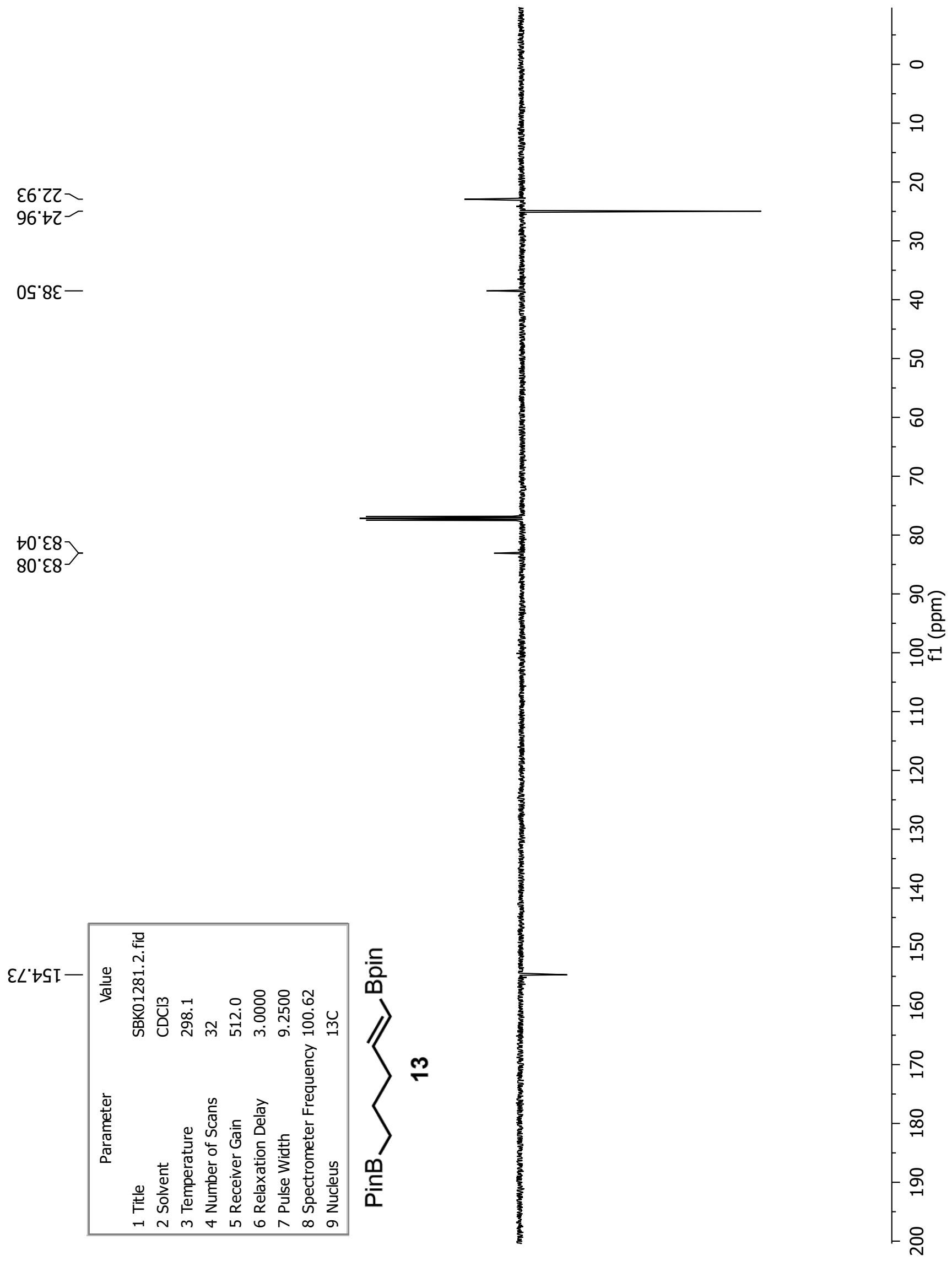


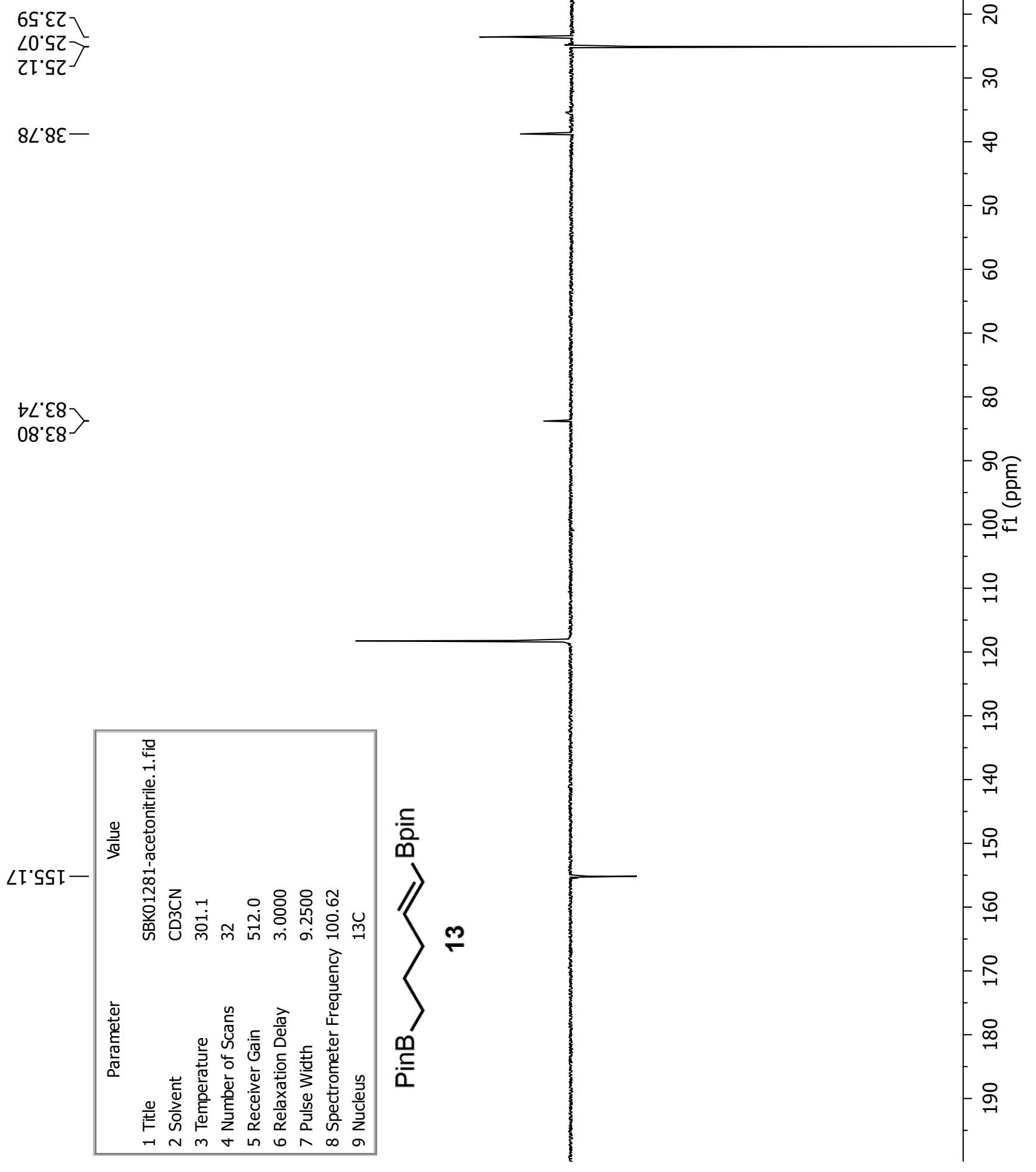




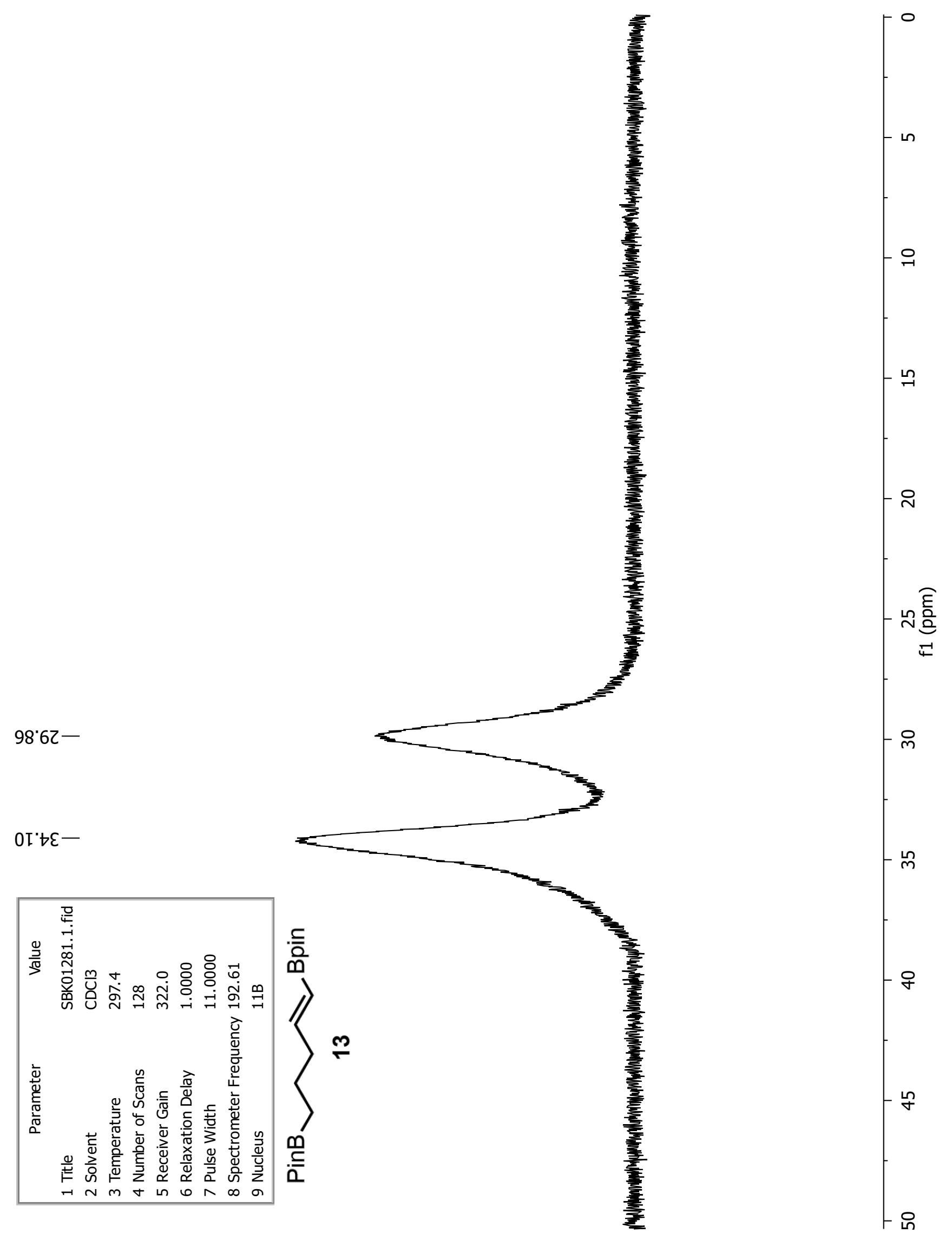




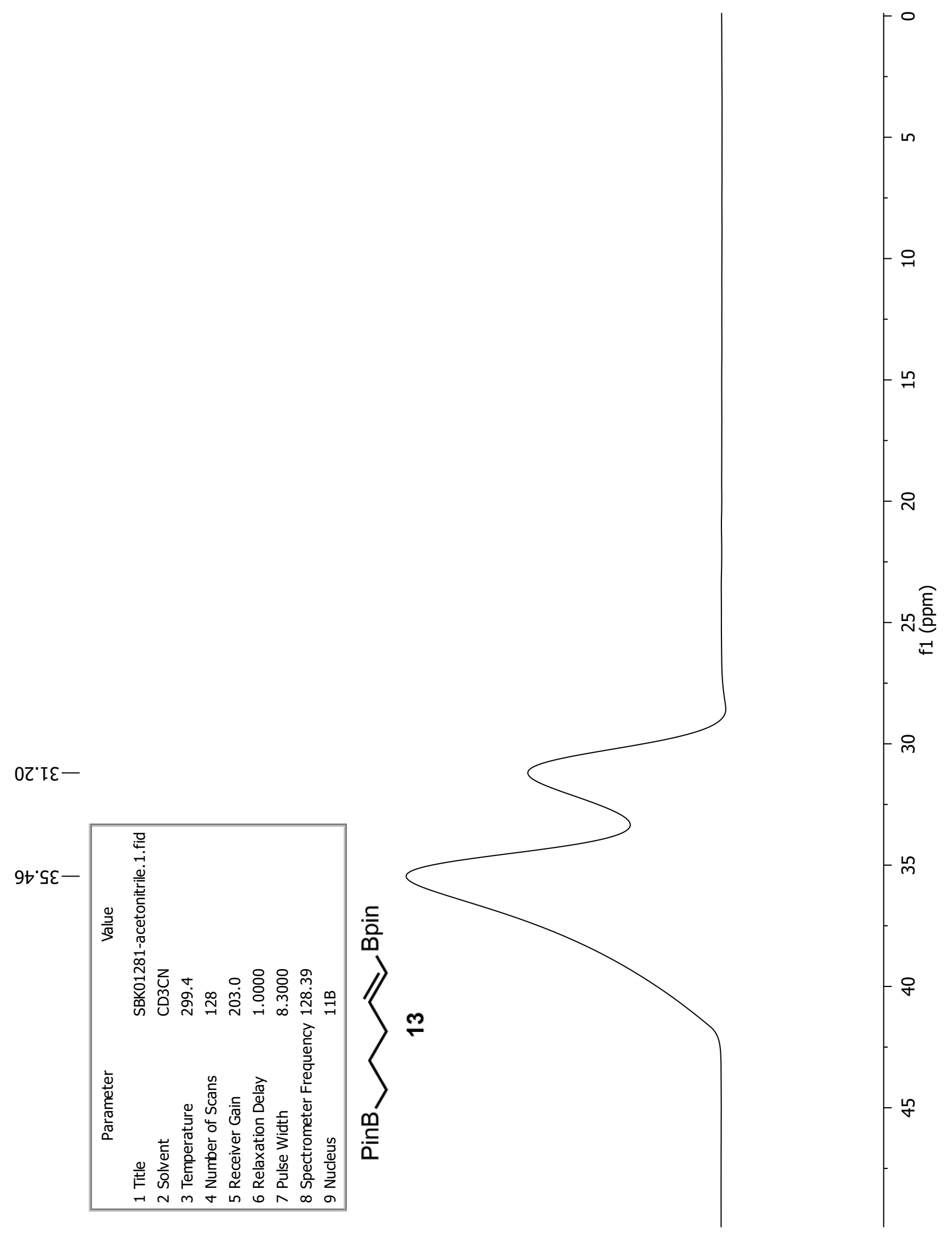




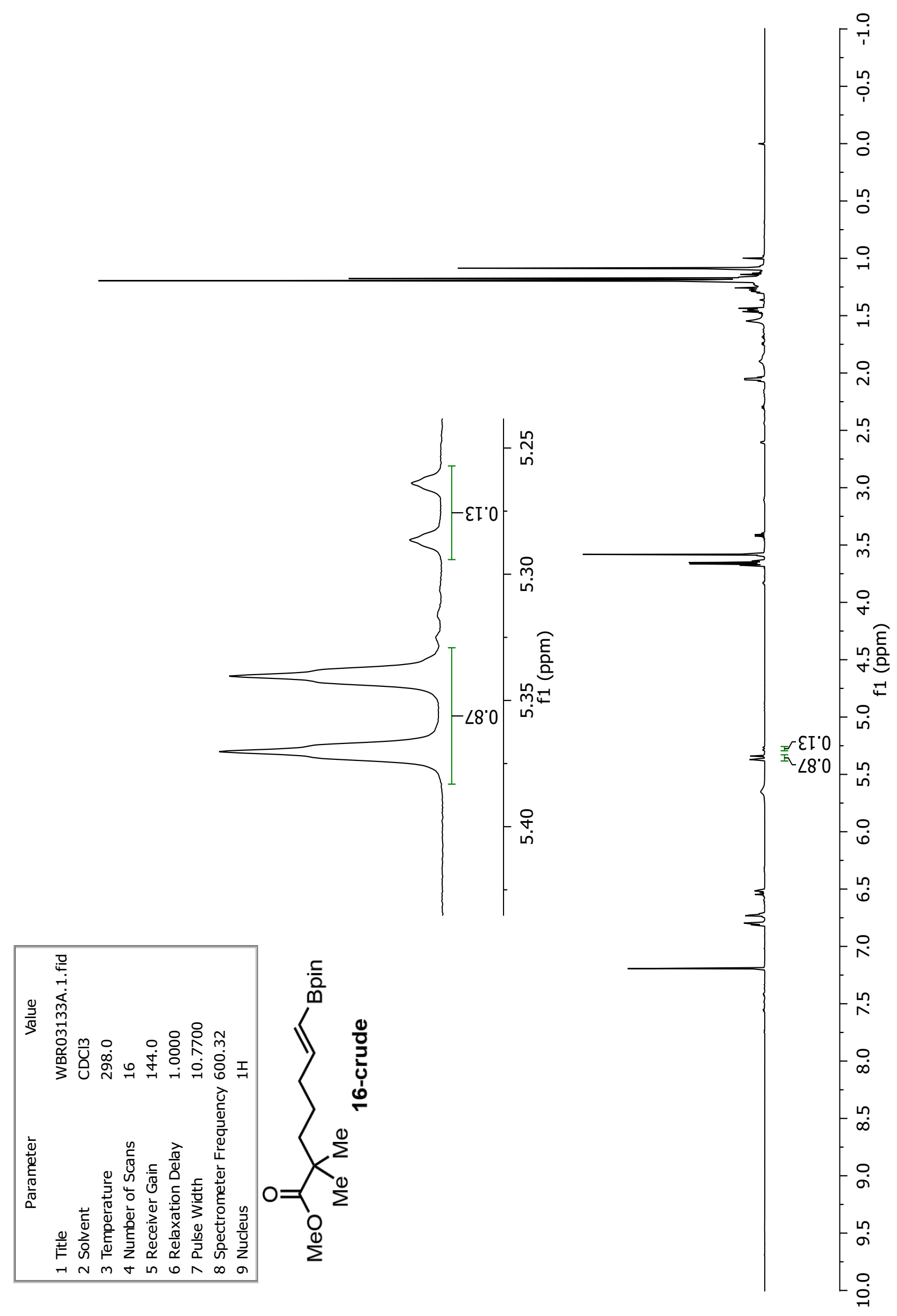




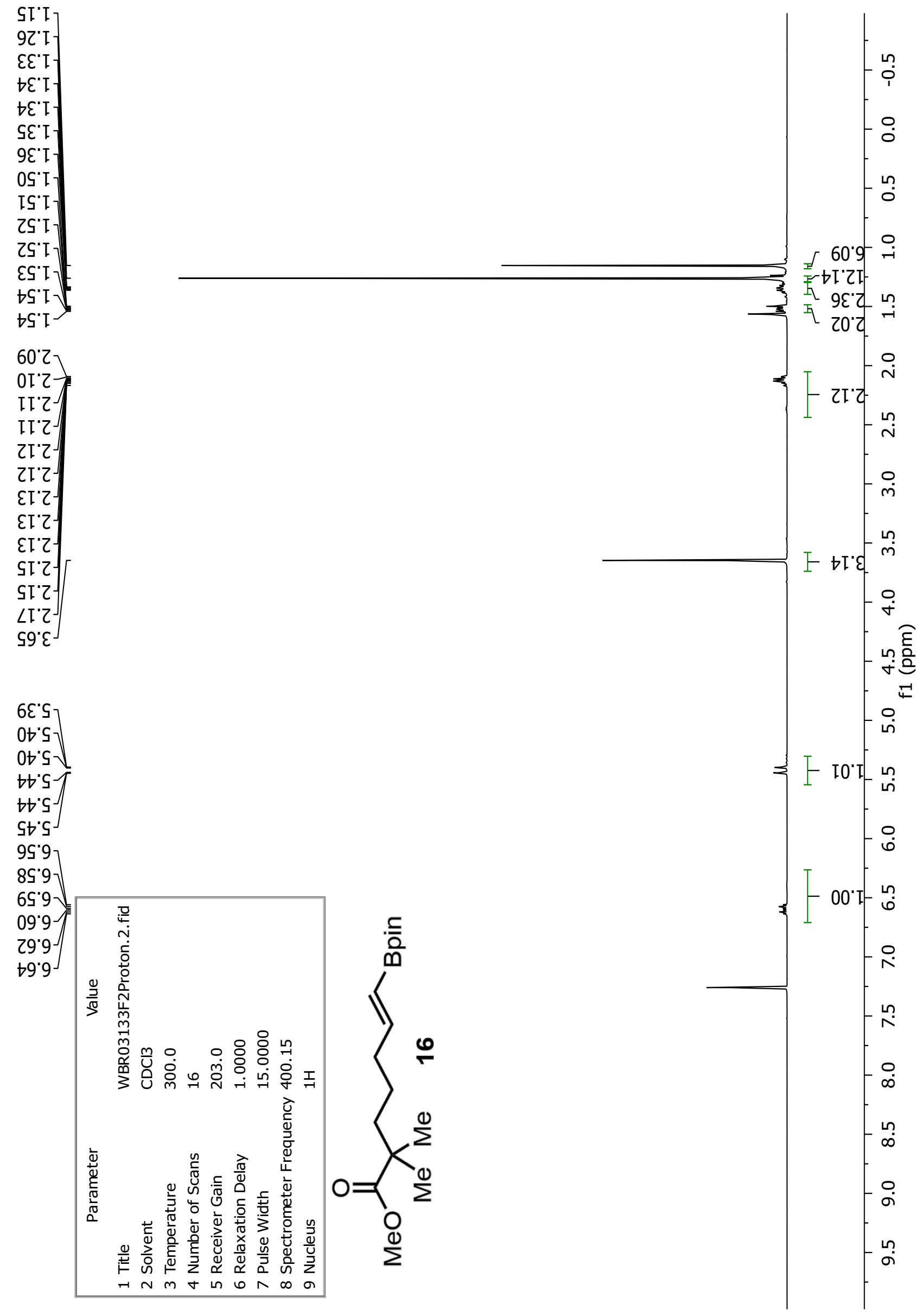




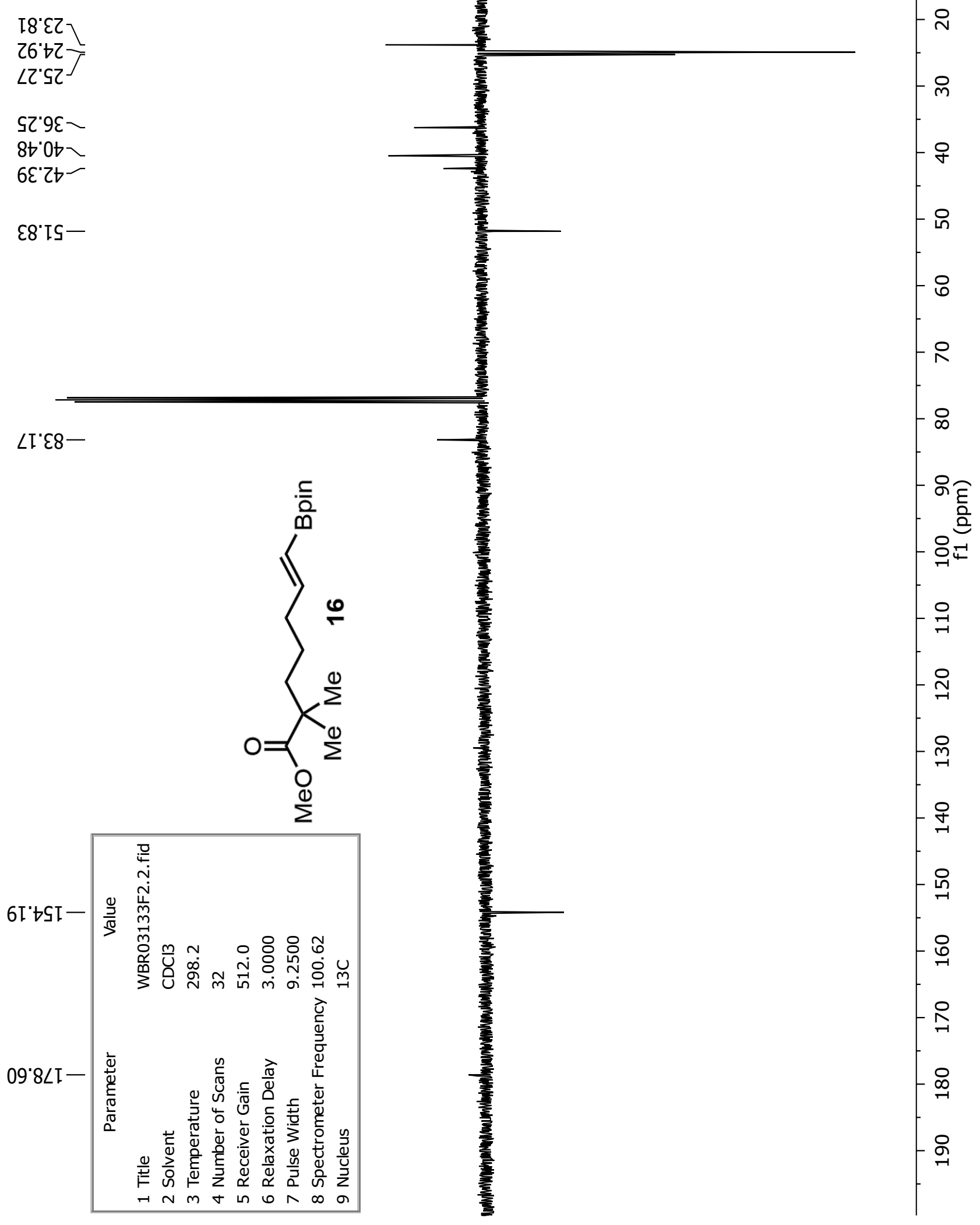




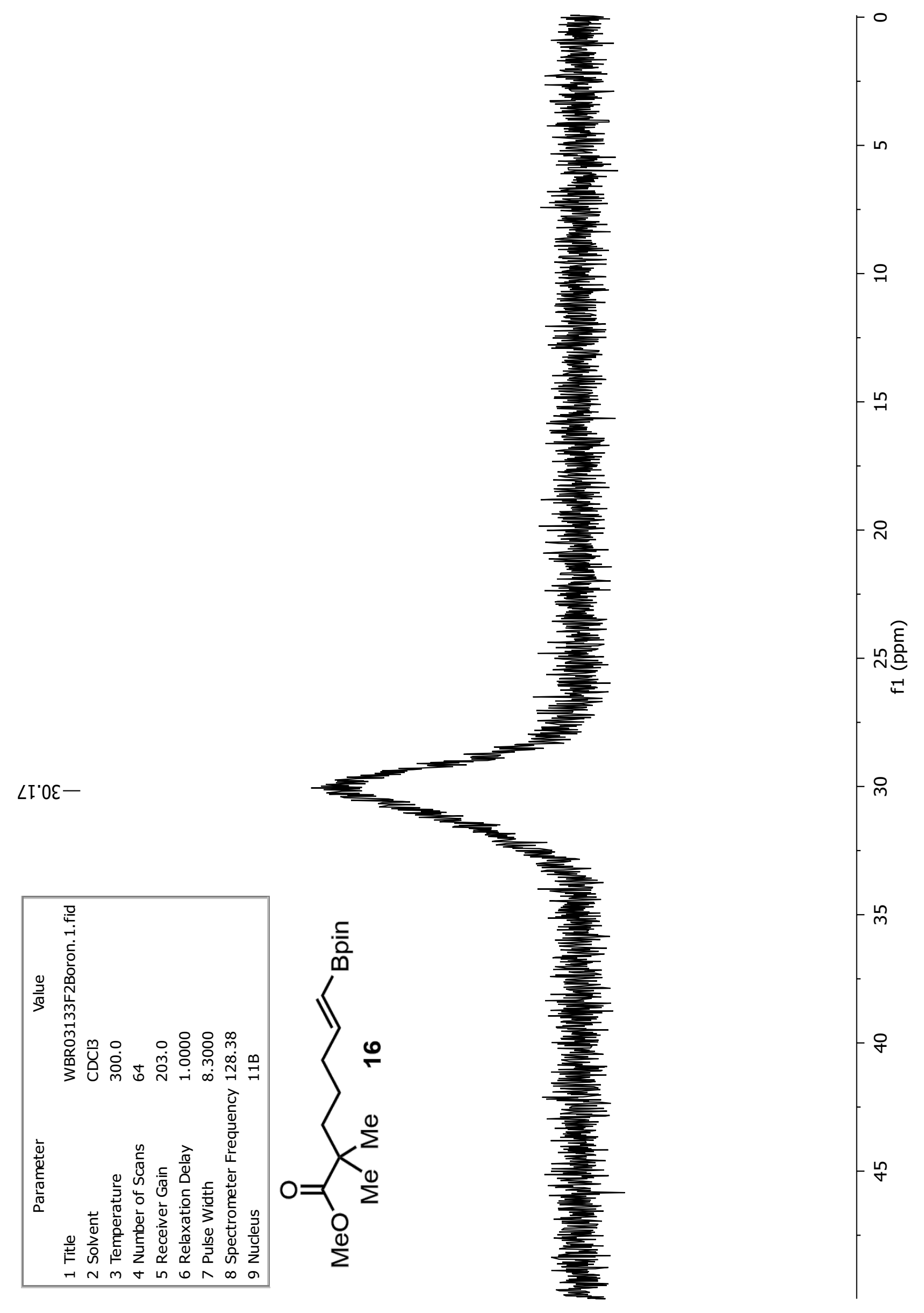




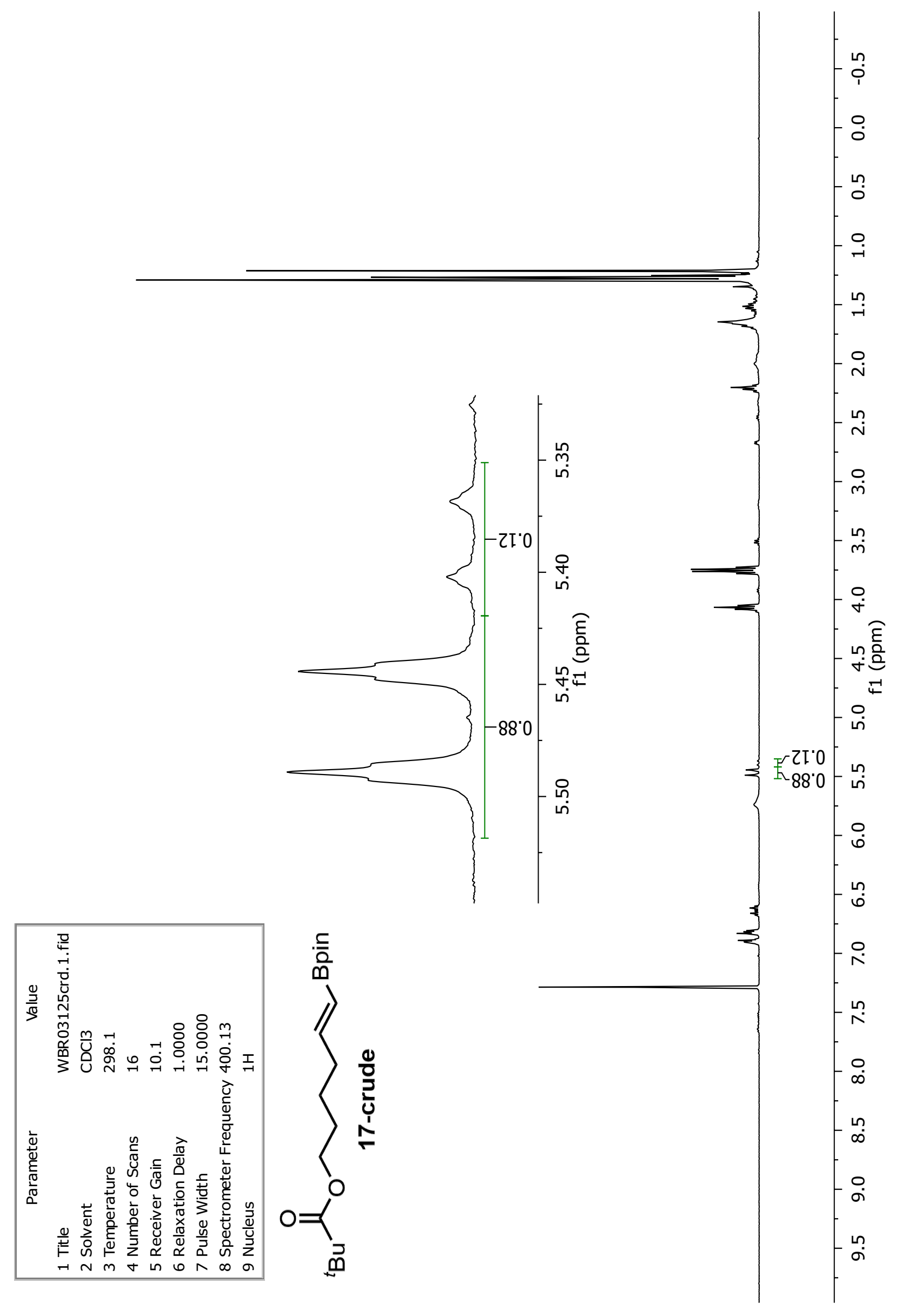




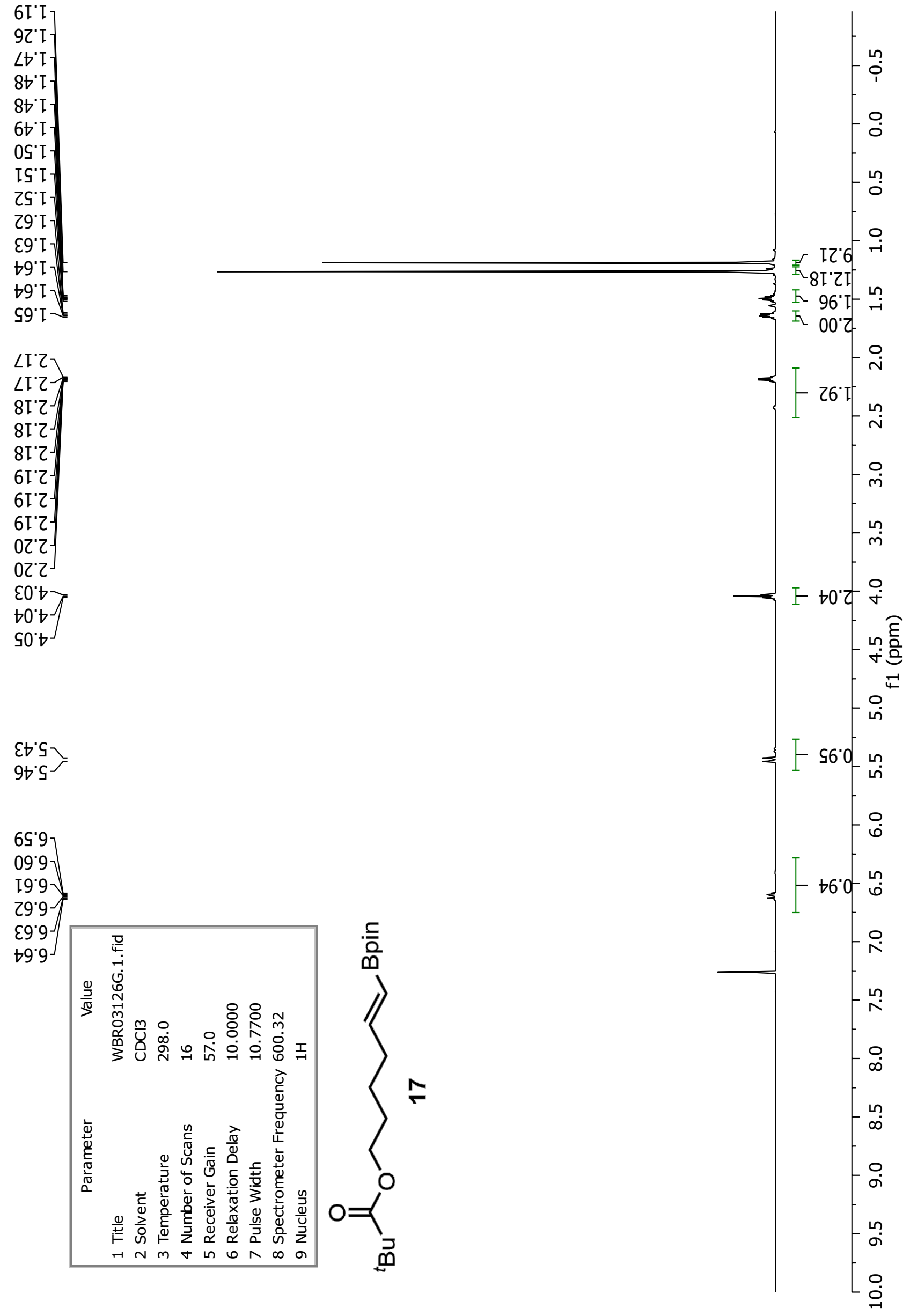




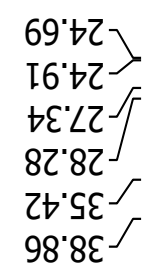

Iย'เ9-

6I'ย8-
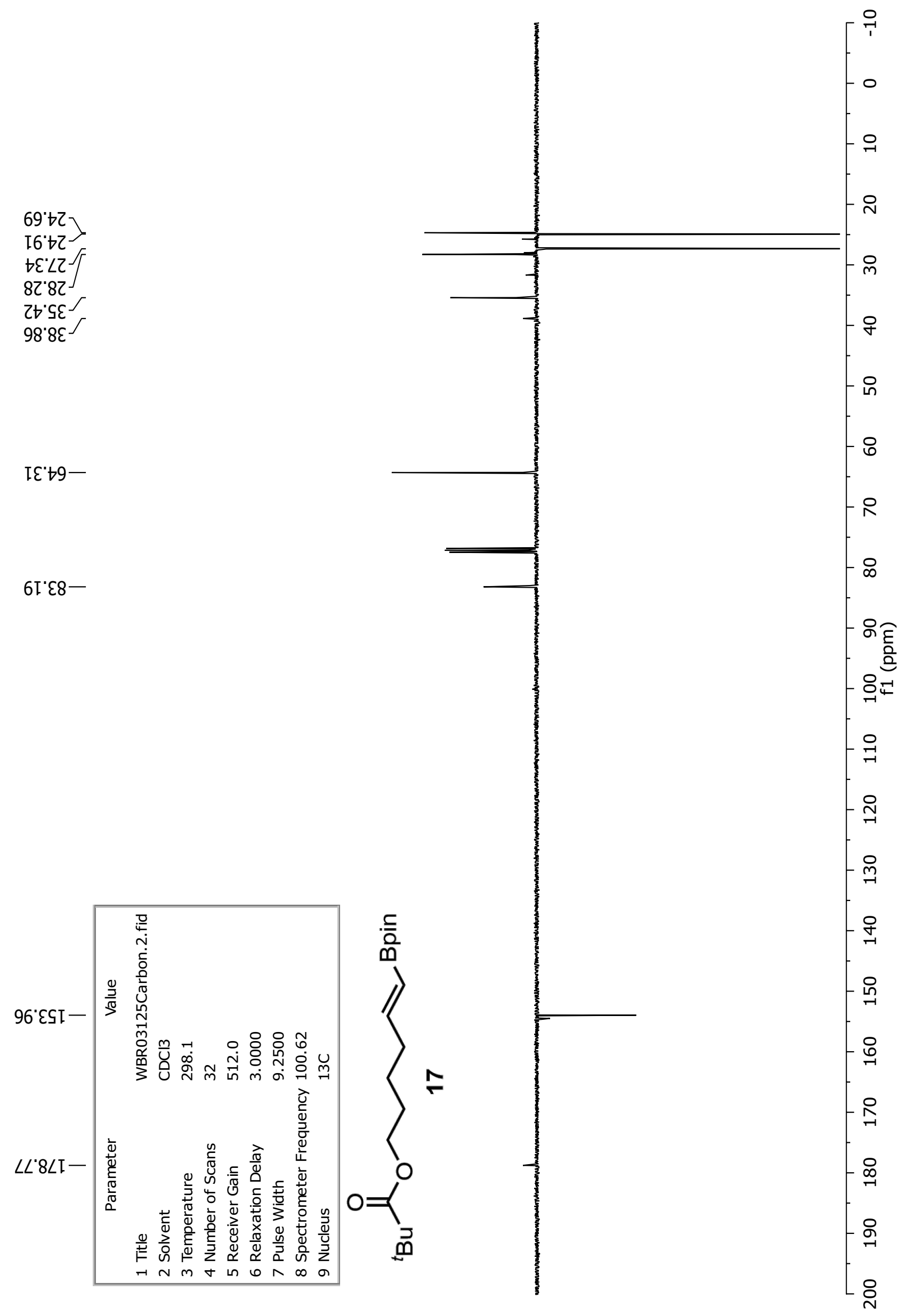


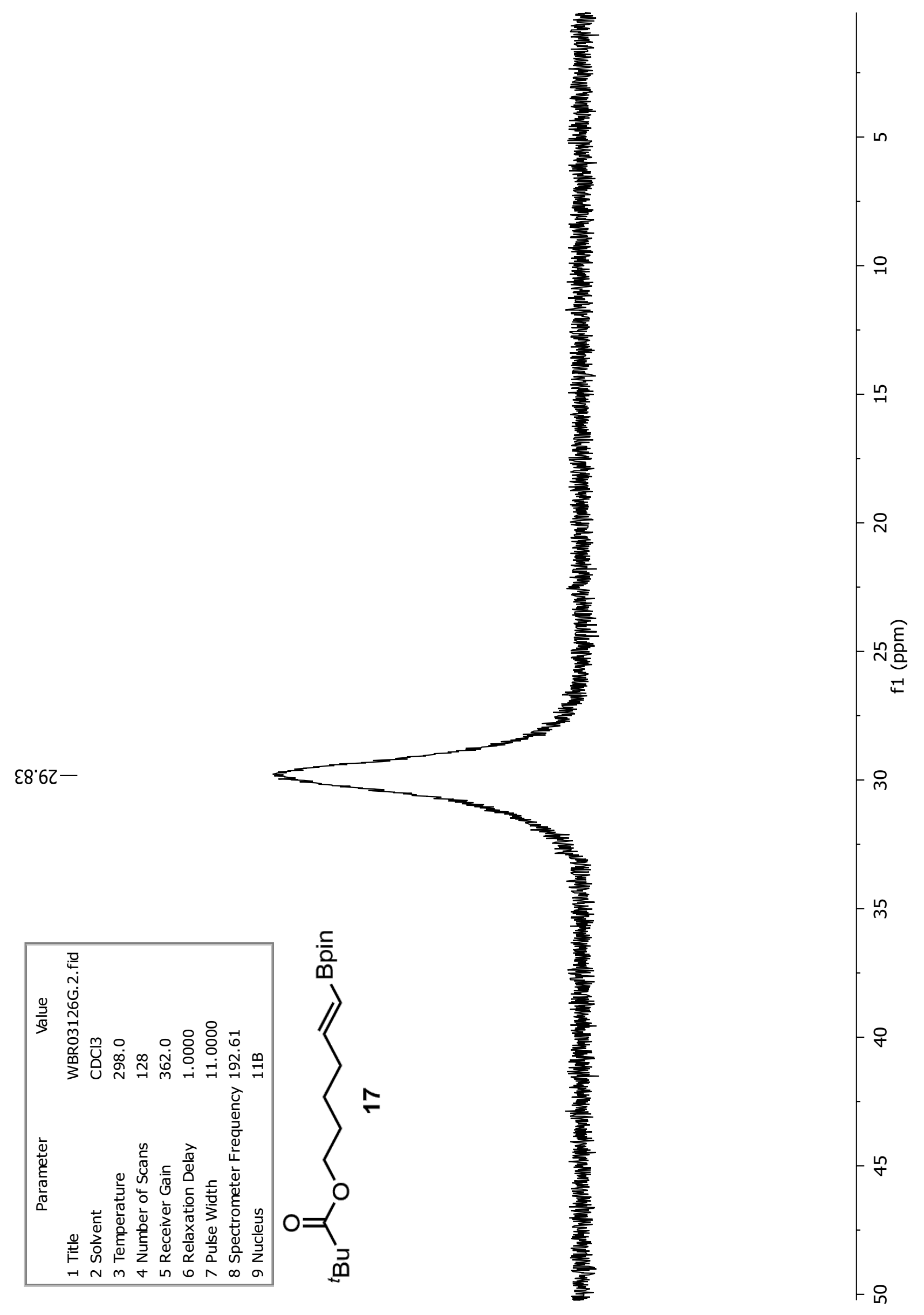




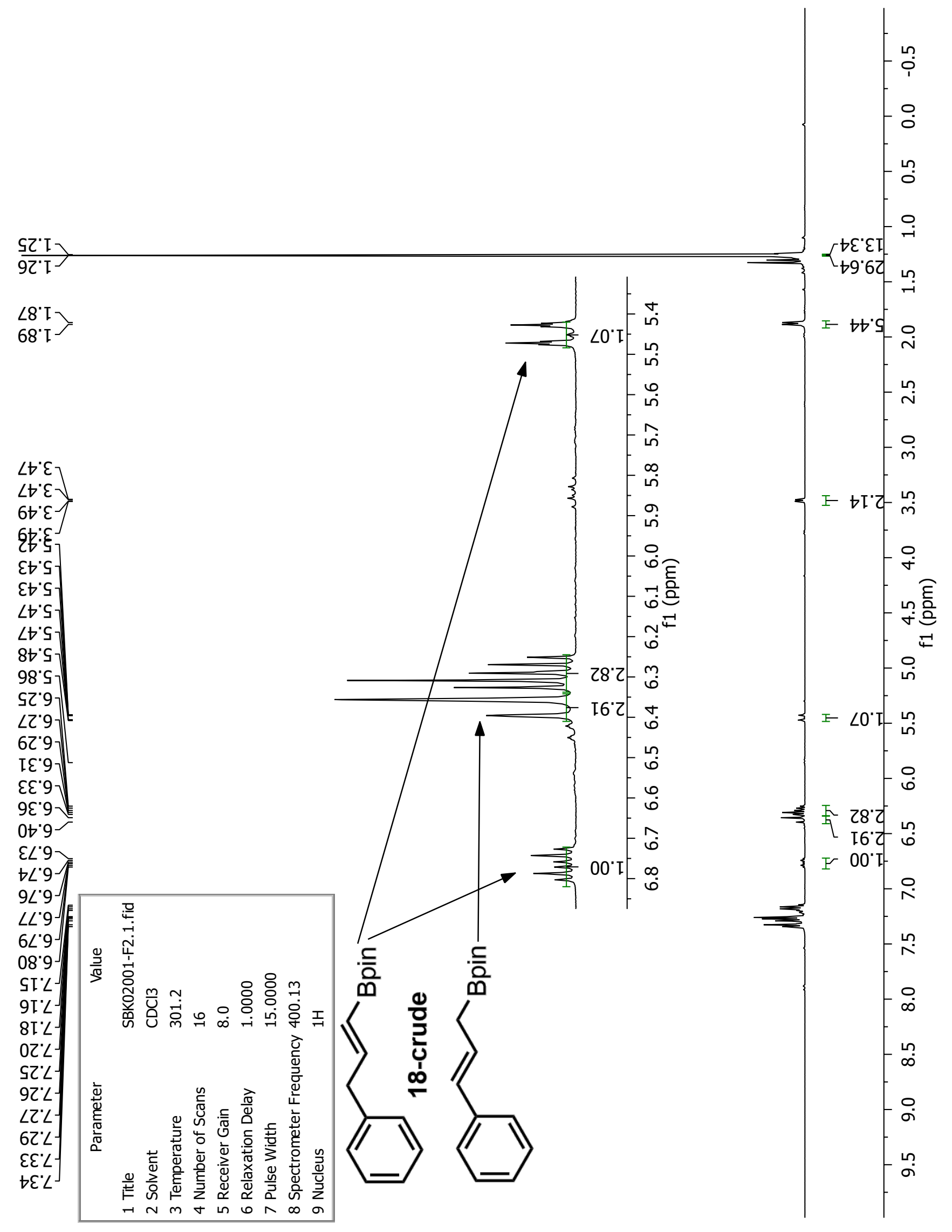




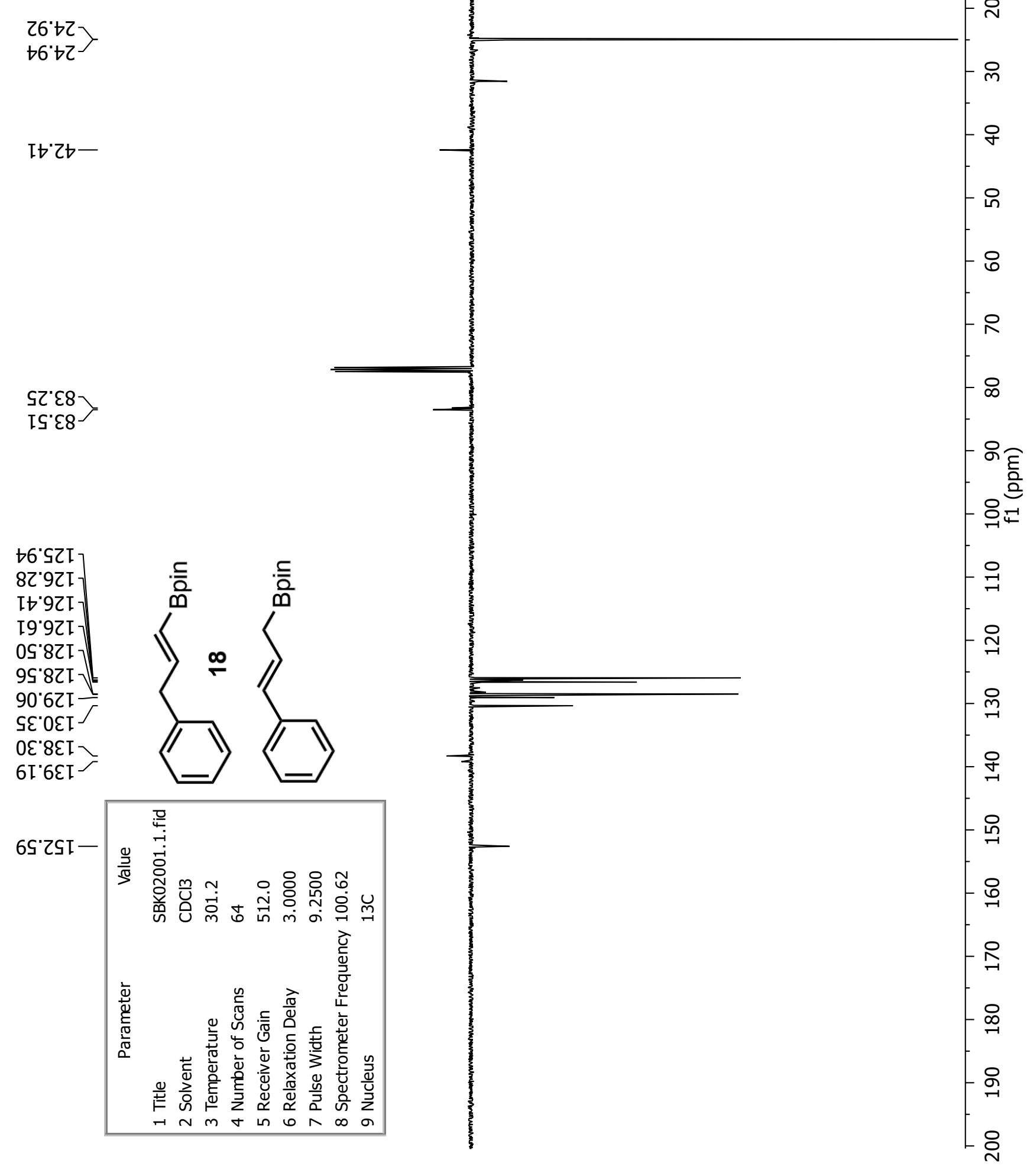


$\varepsilon \varpi^{\circ} 0 \varepsilon-$

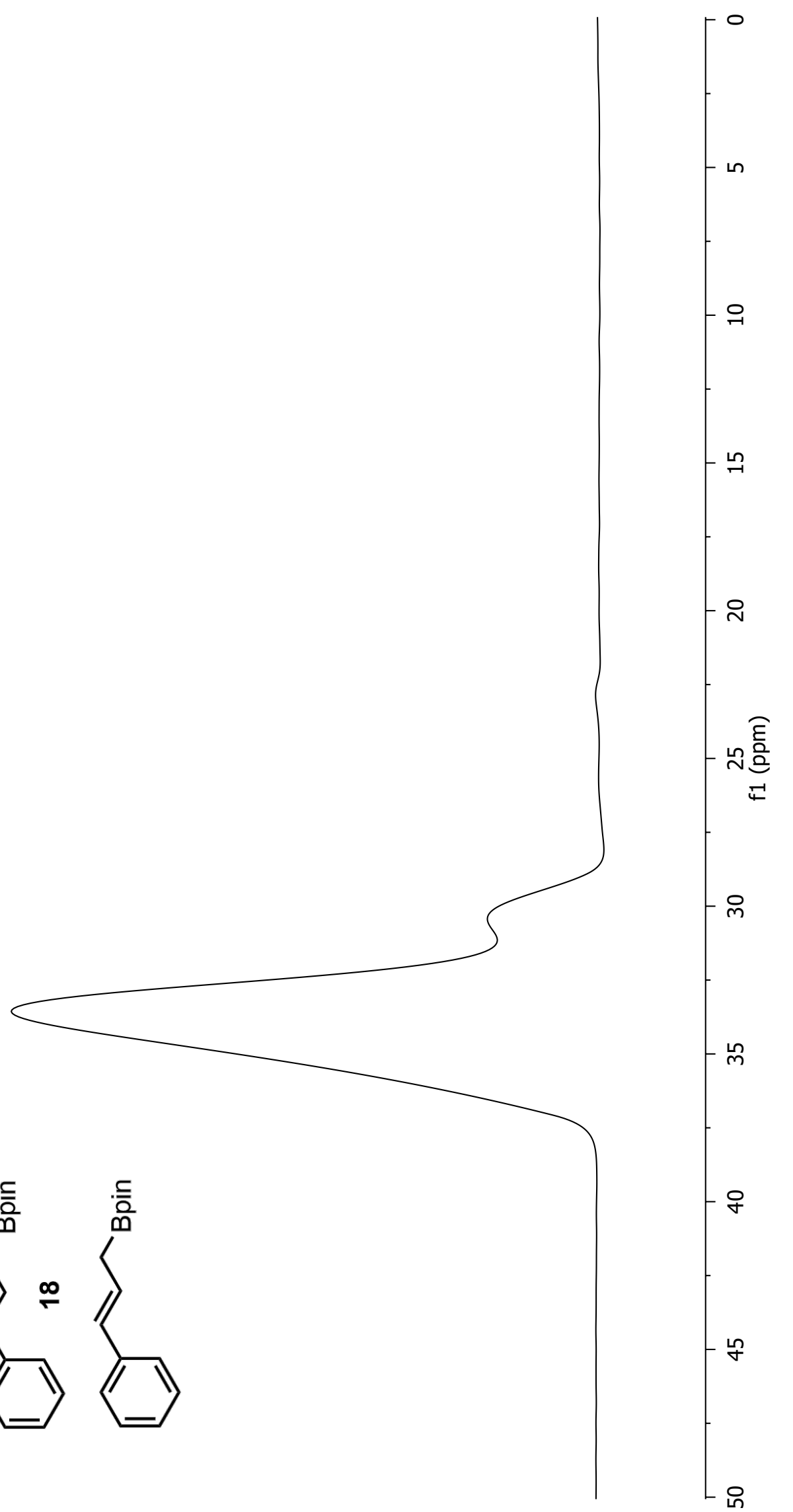




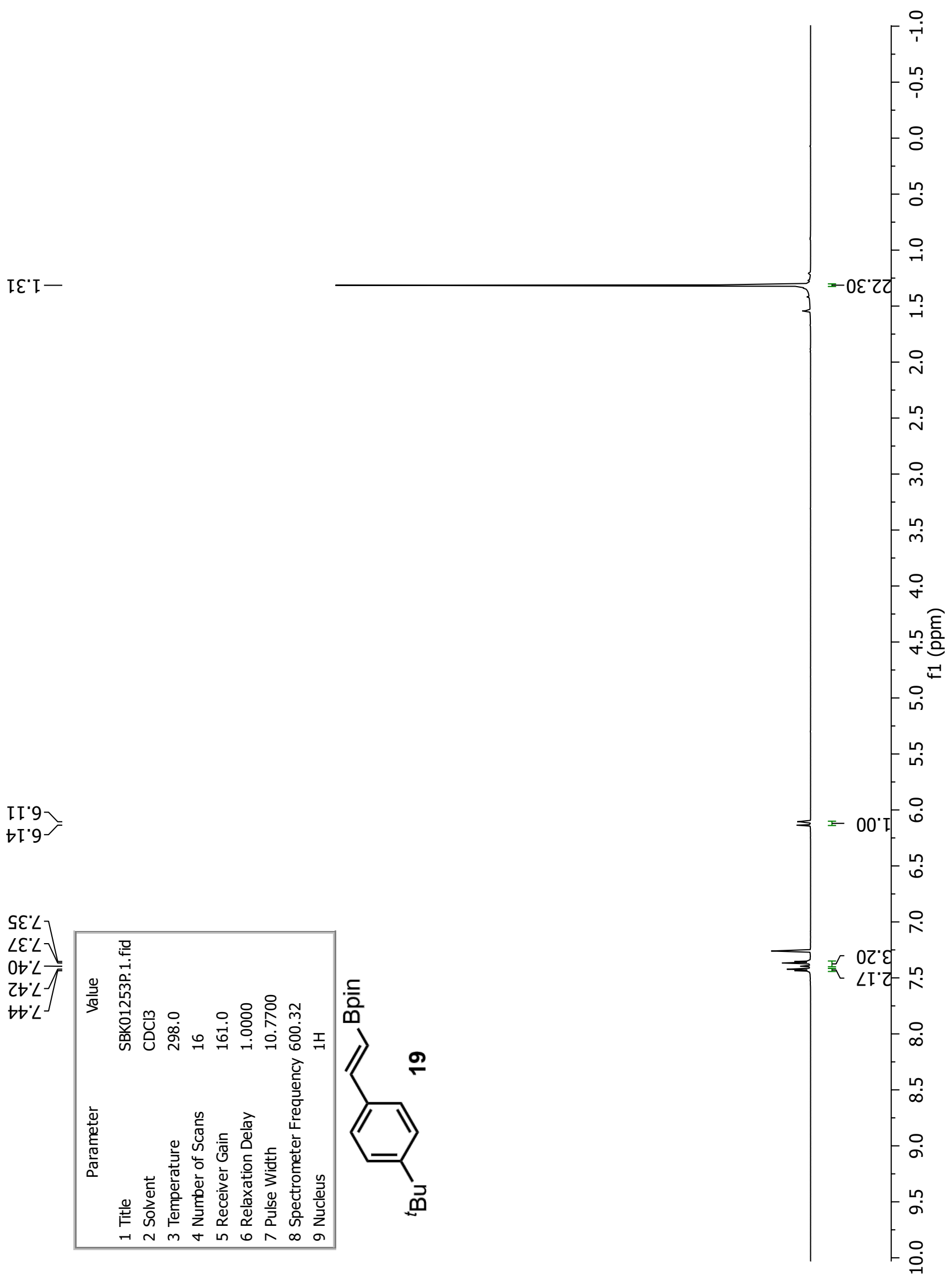



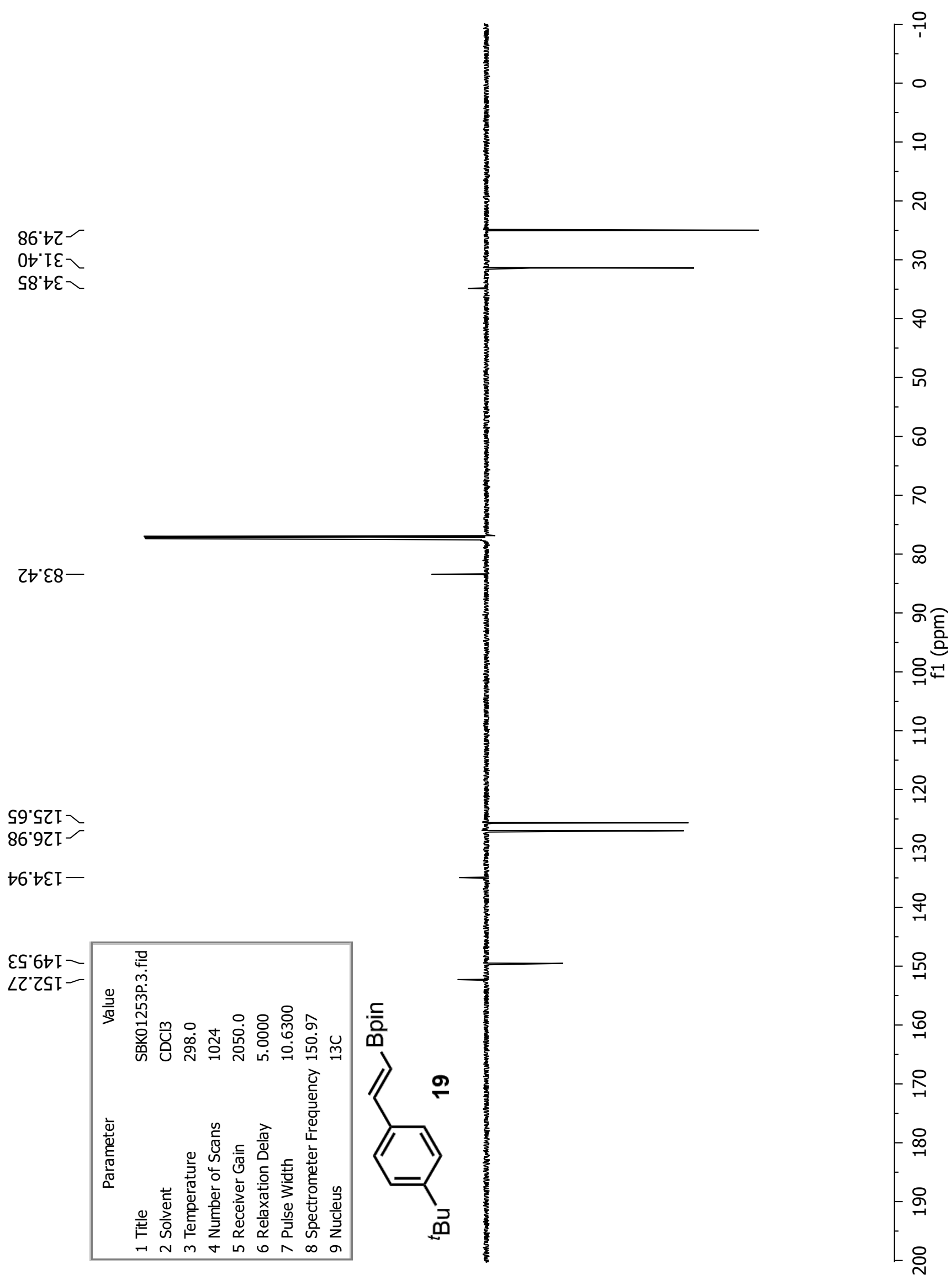


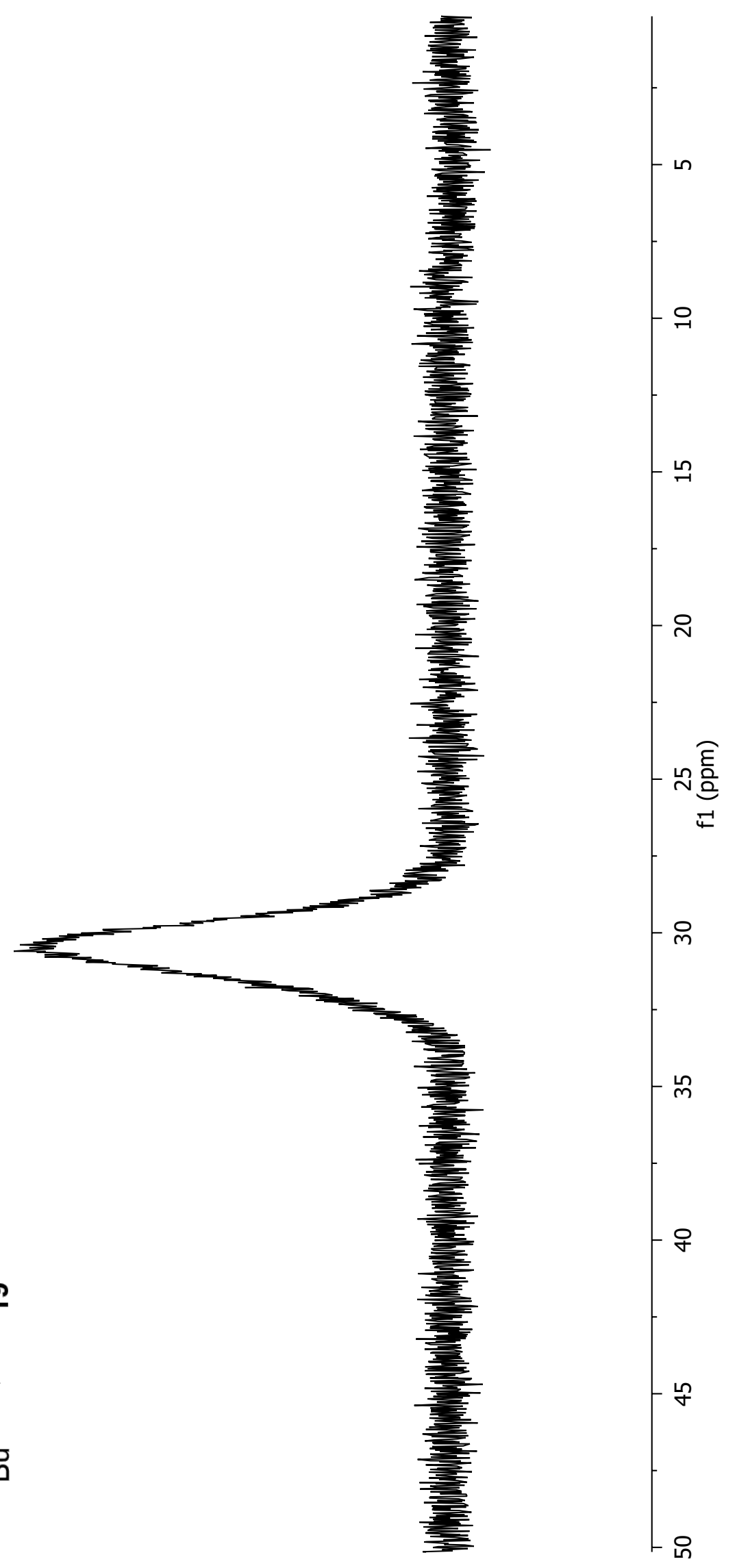




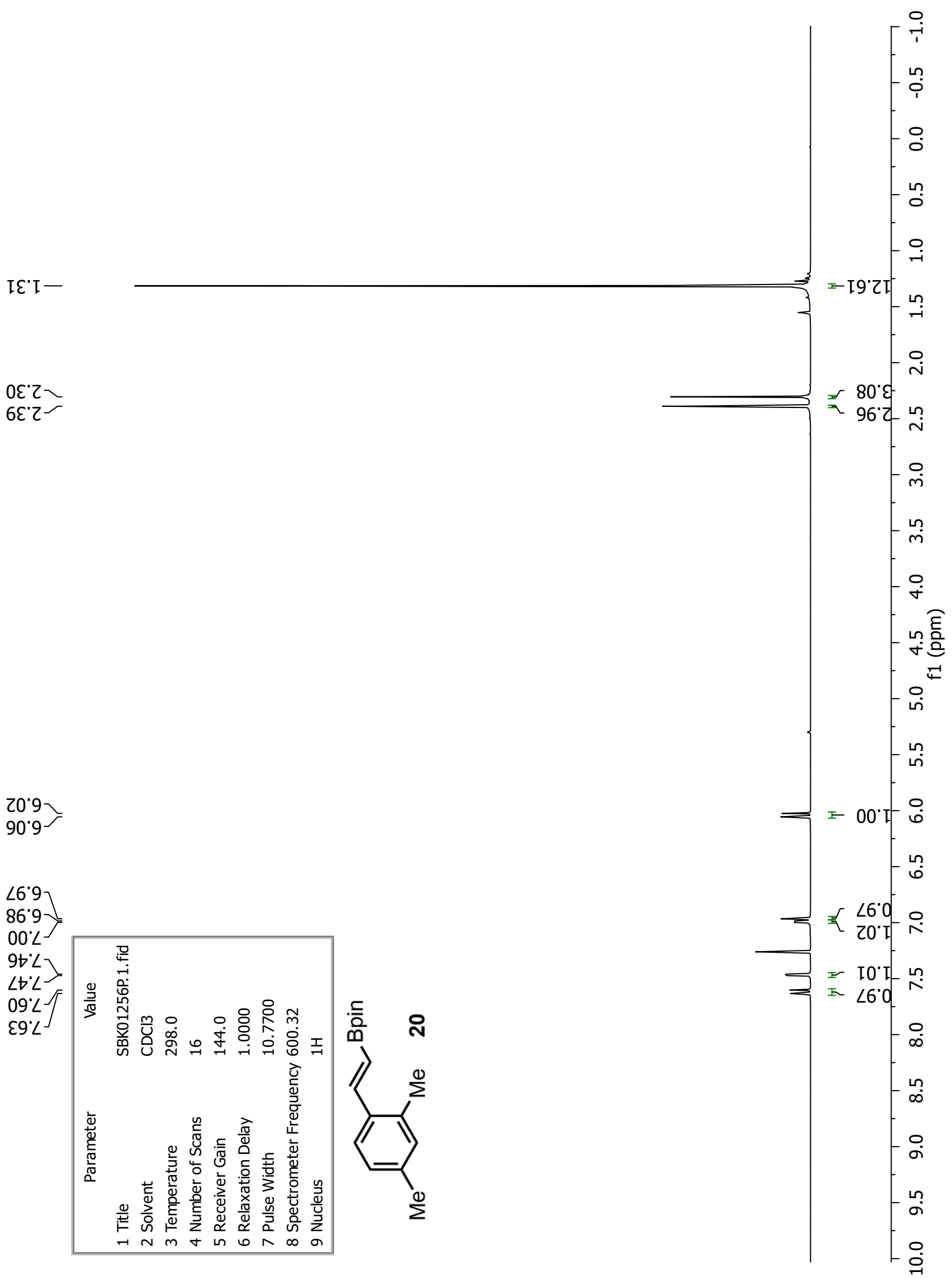




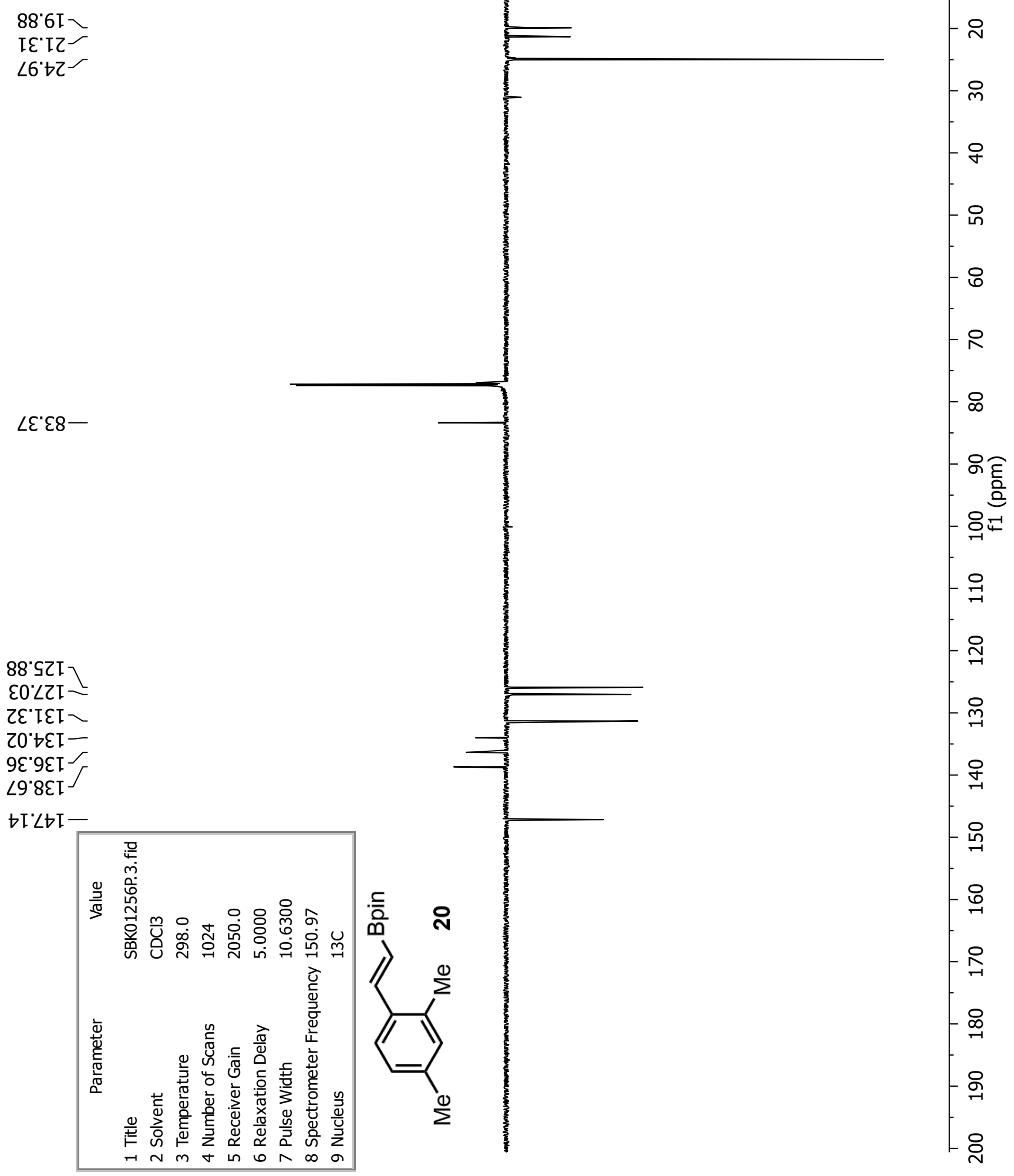




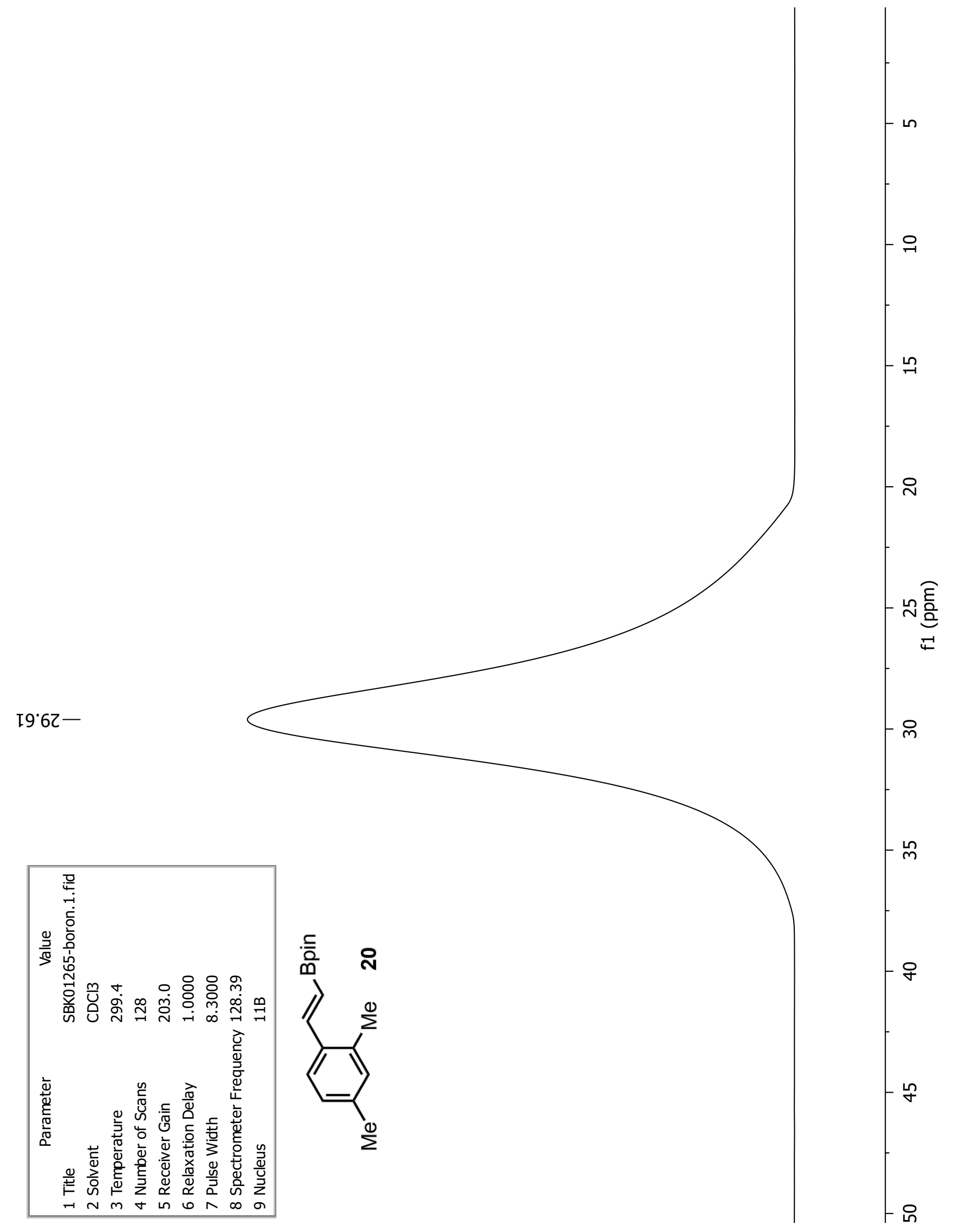




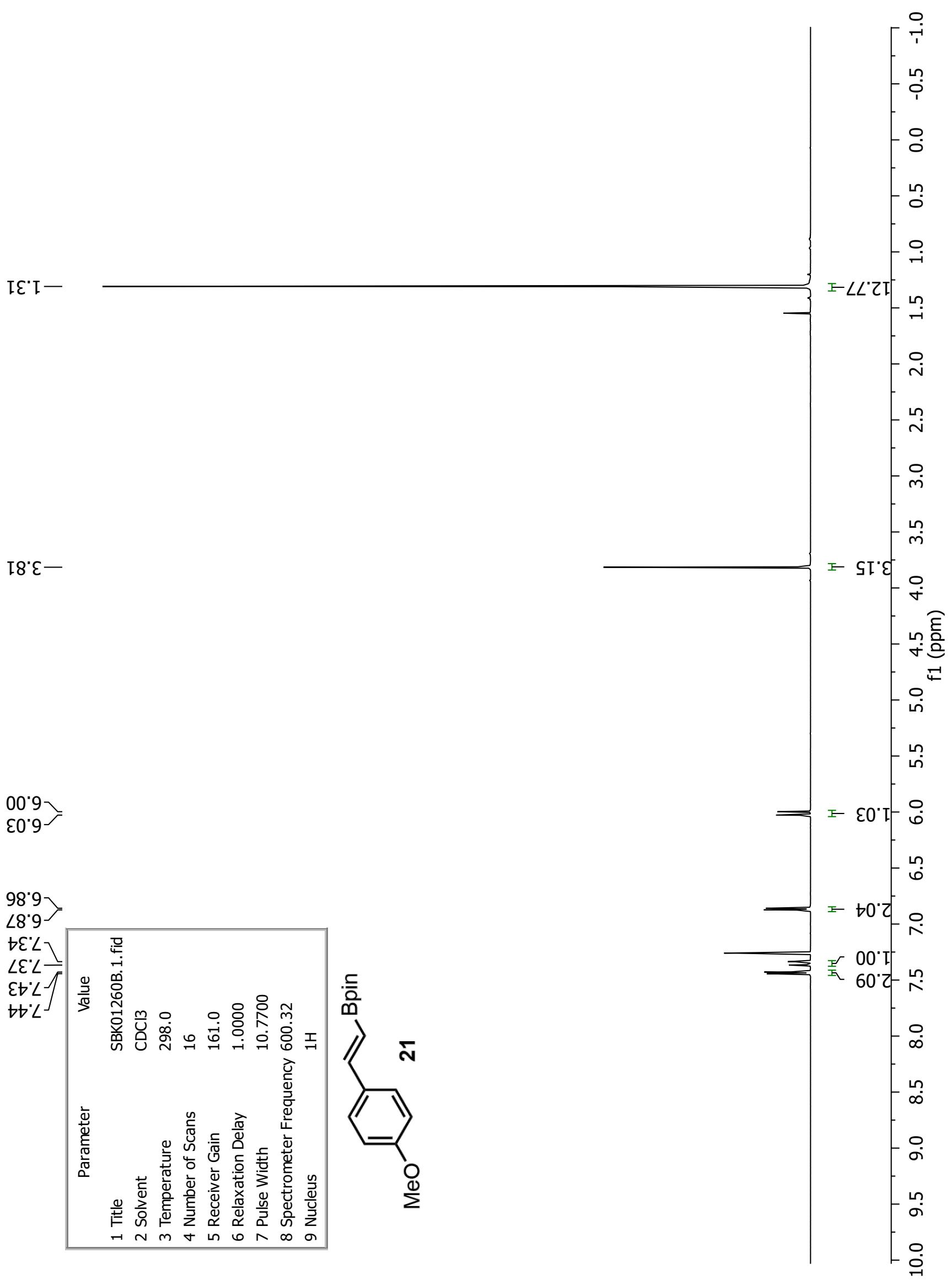




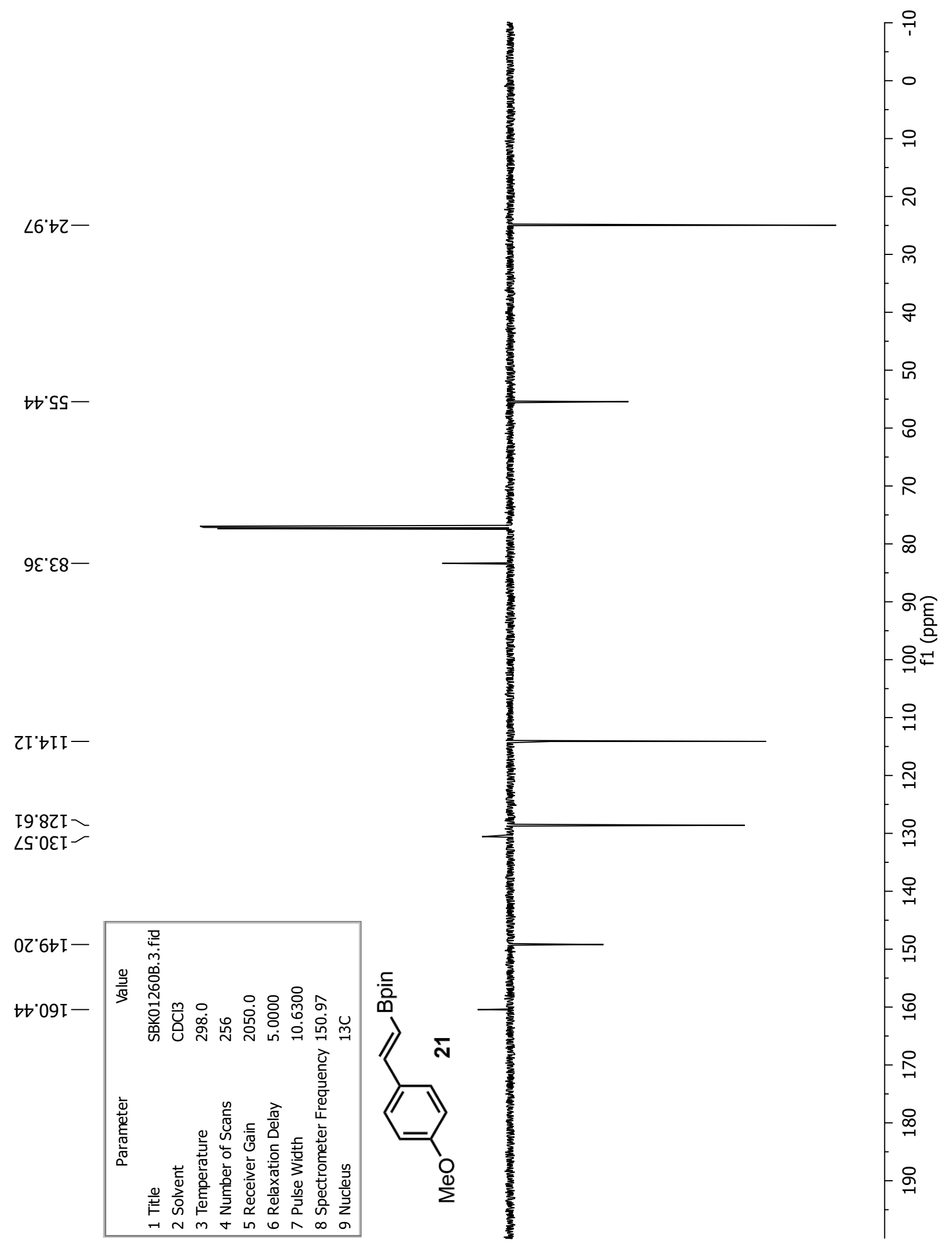



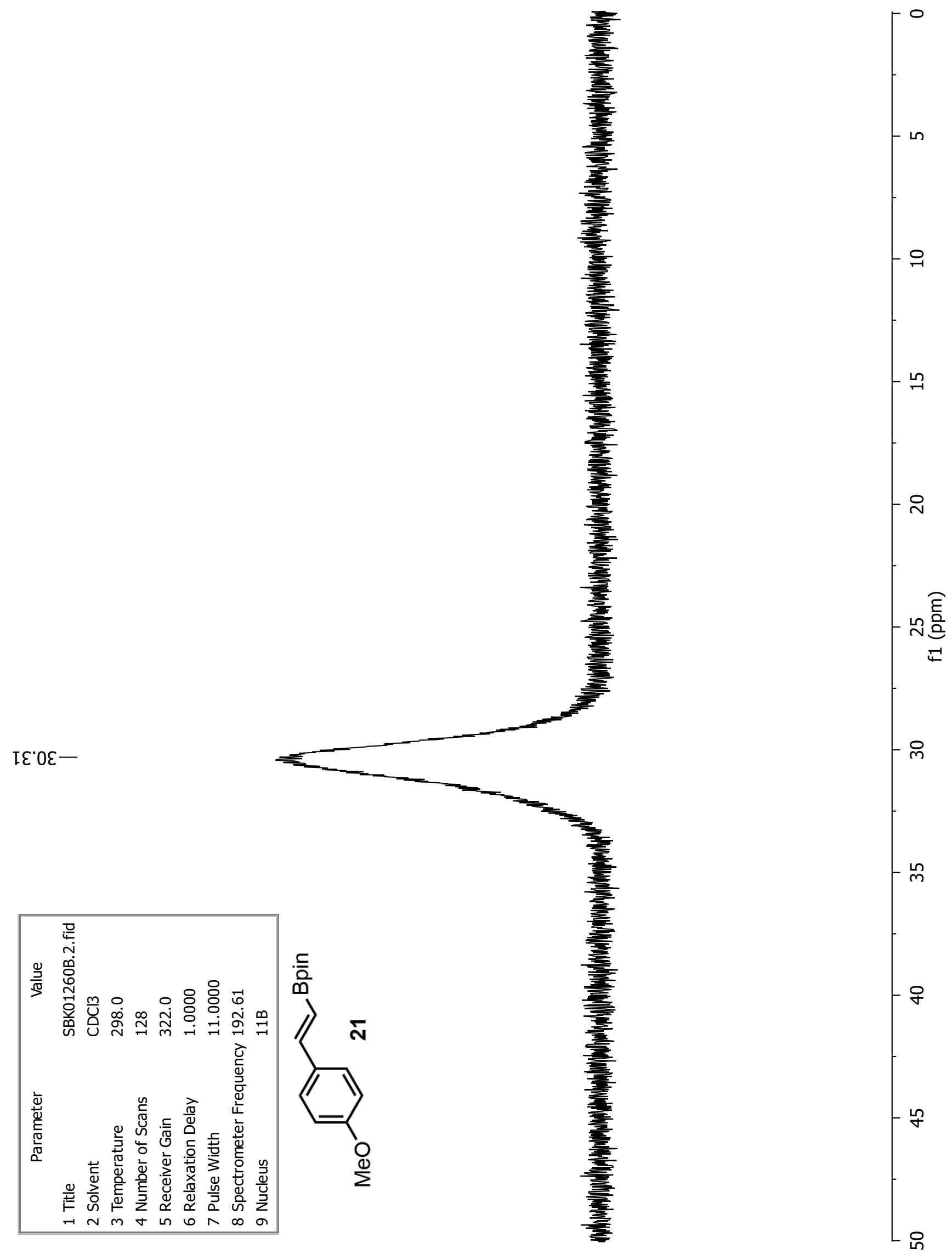

\$109 


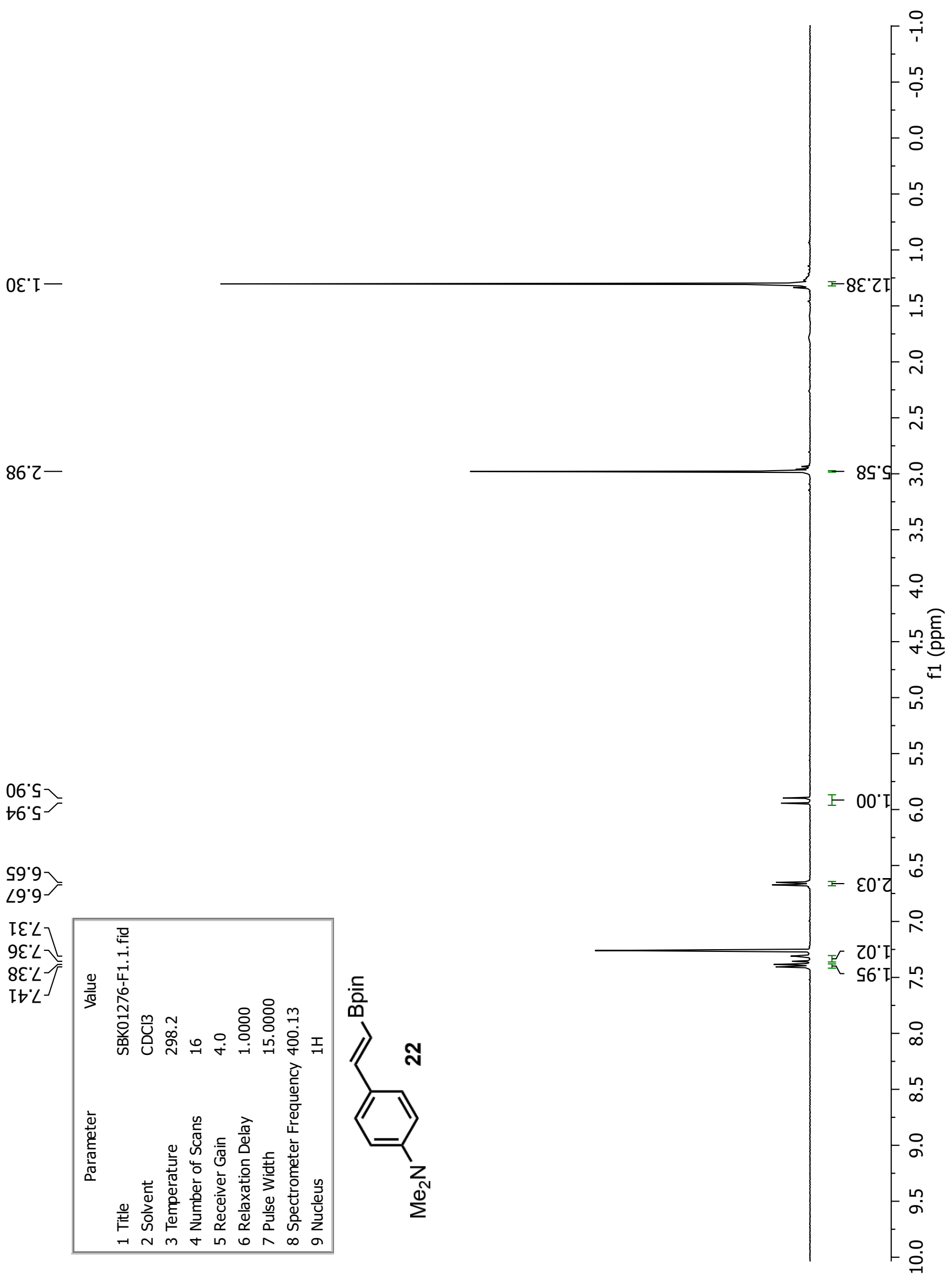



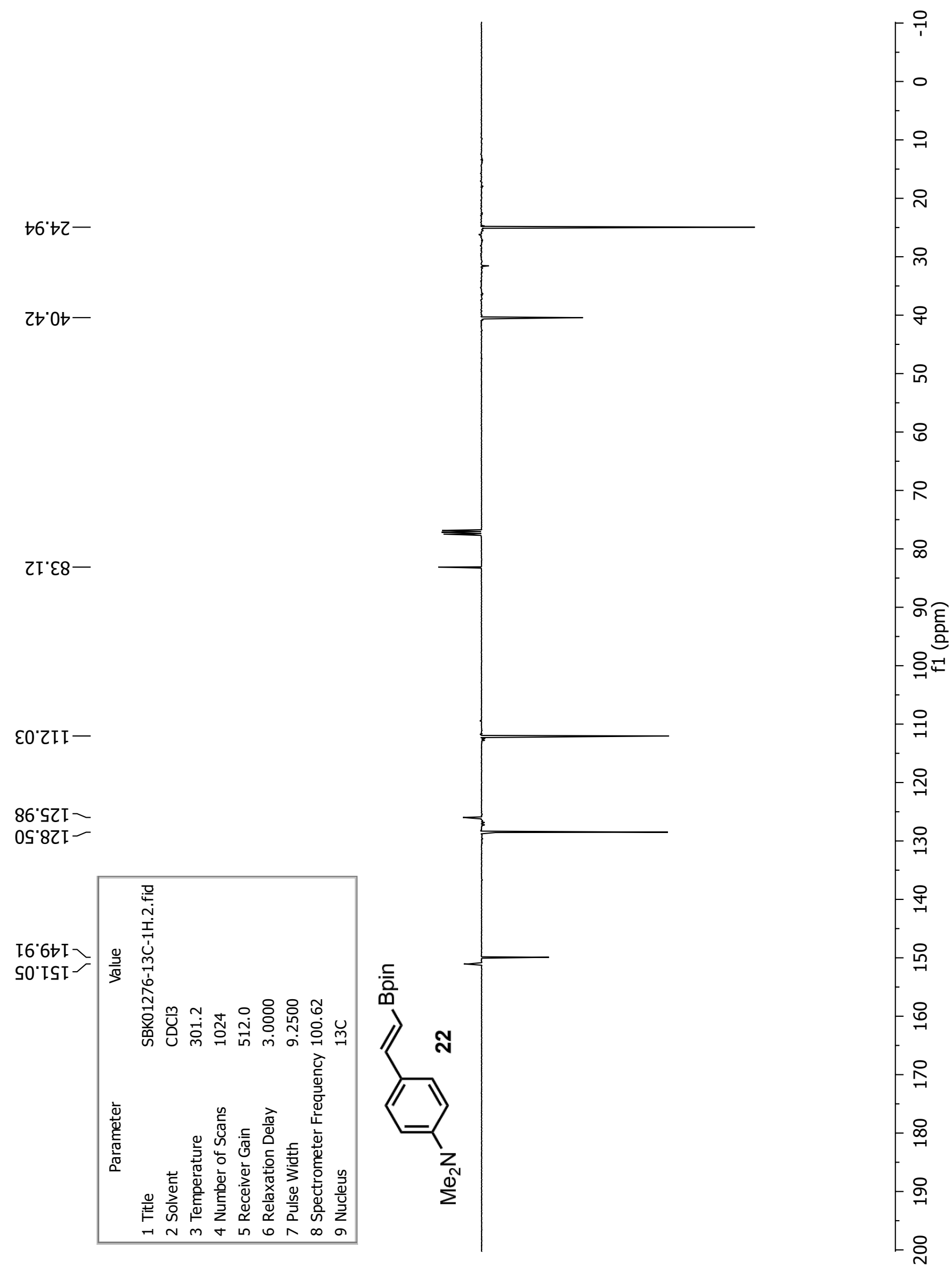


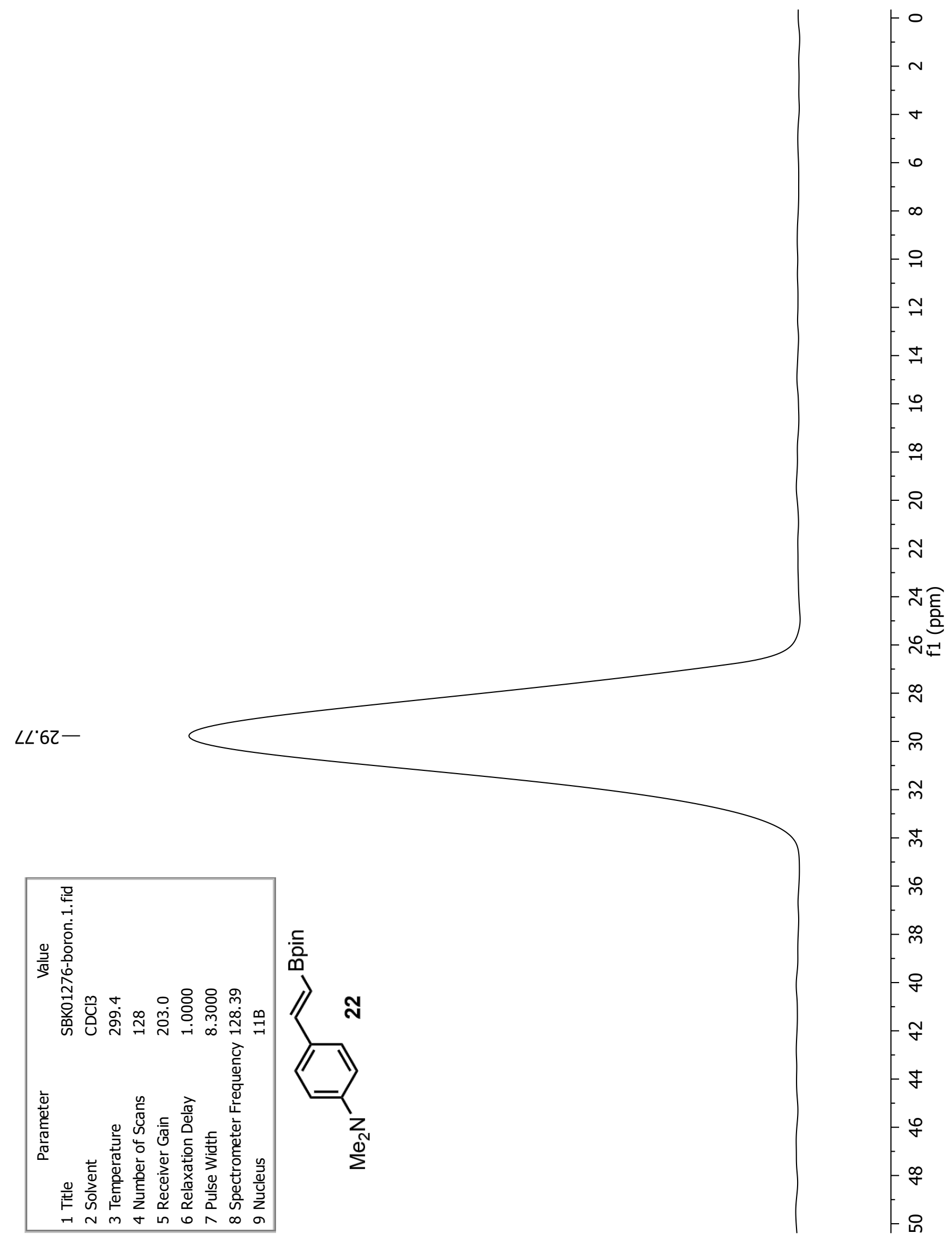




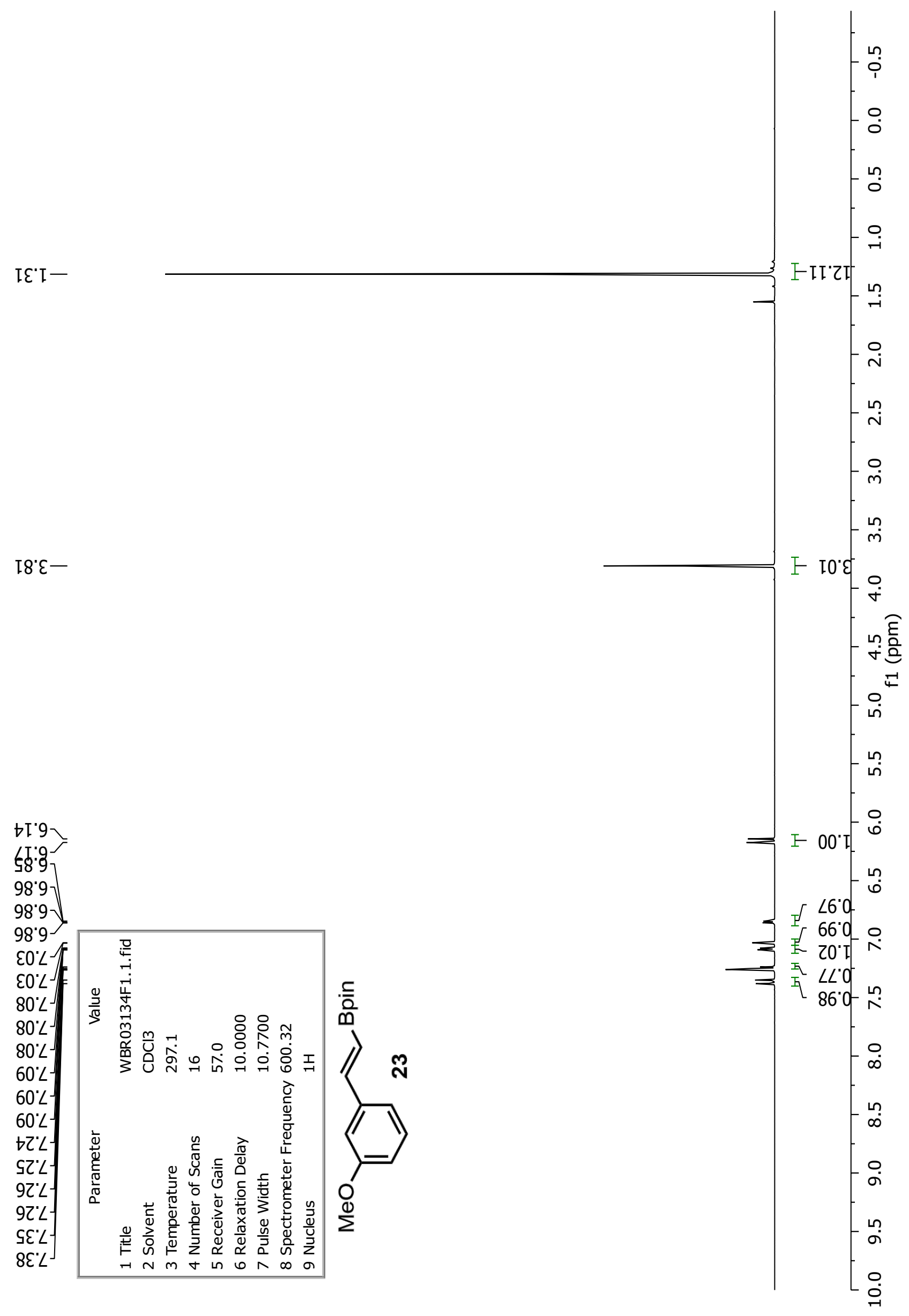




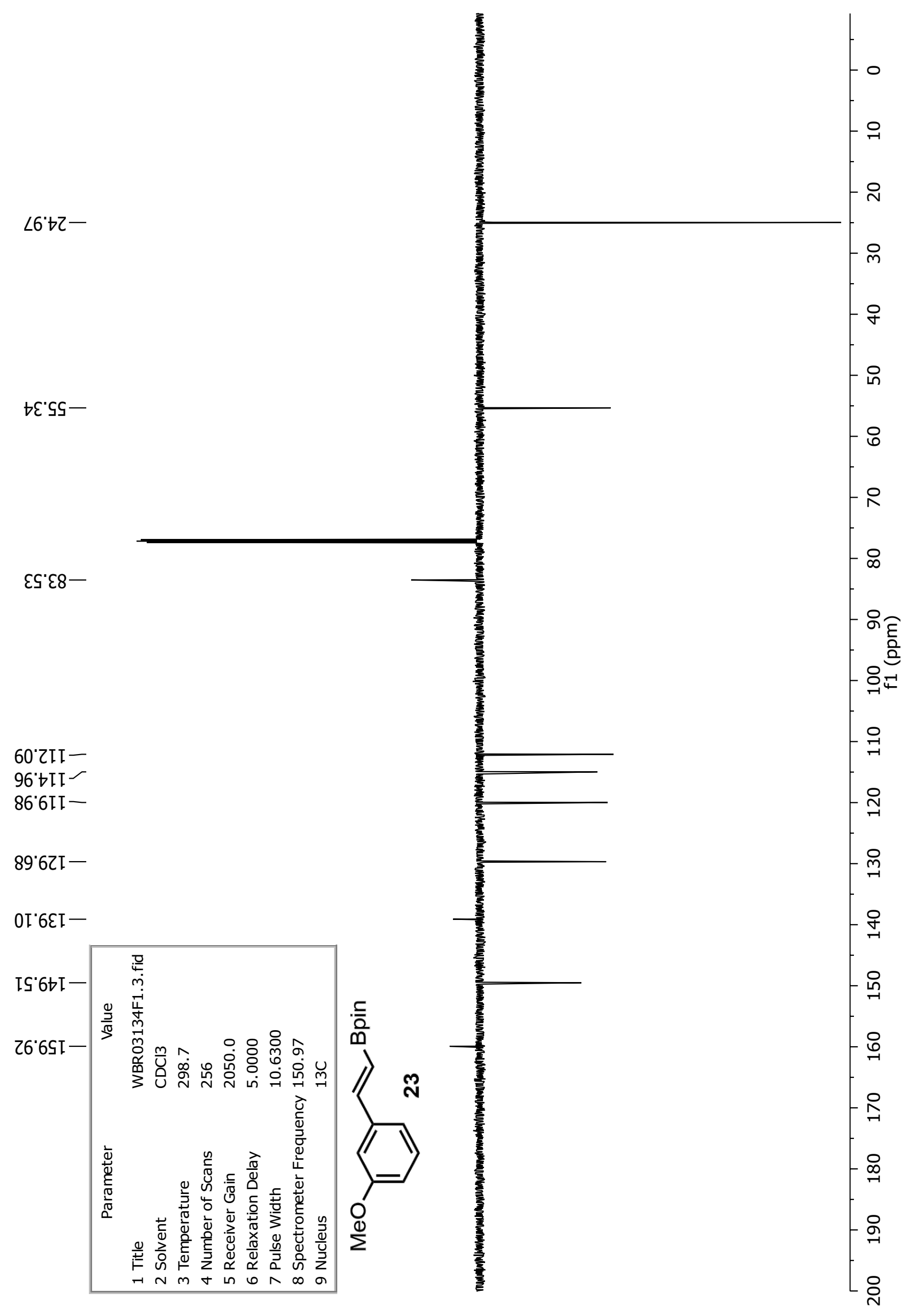




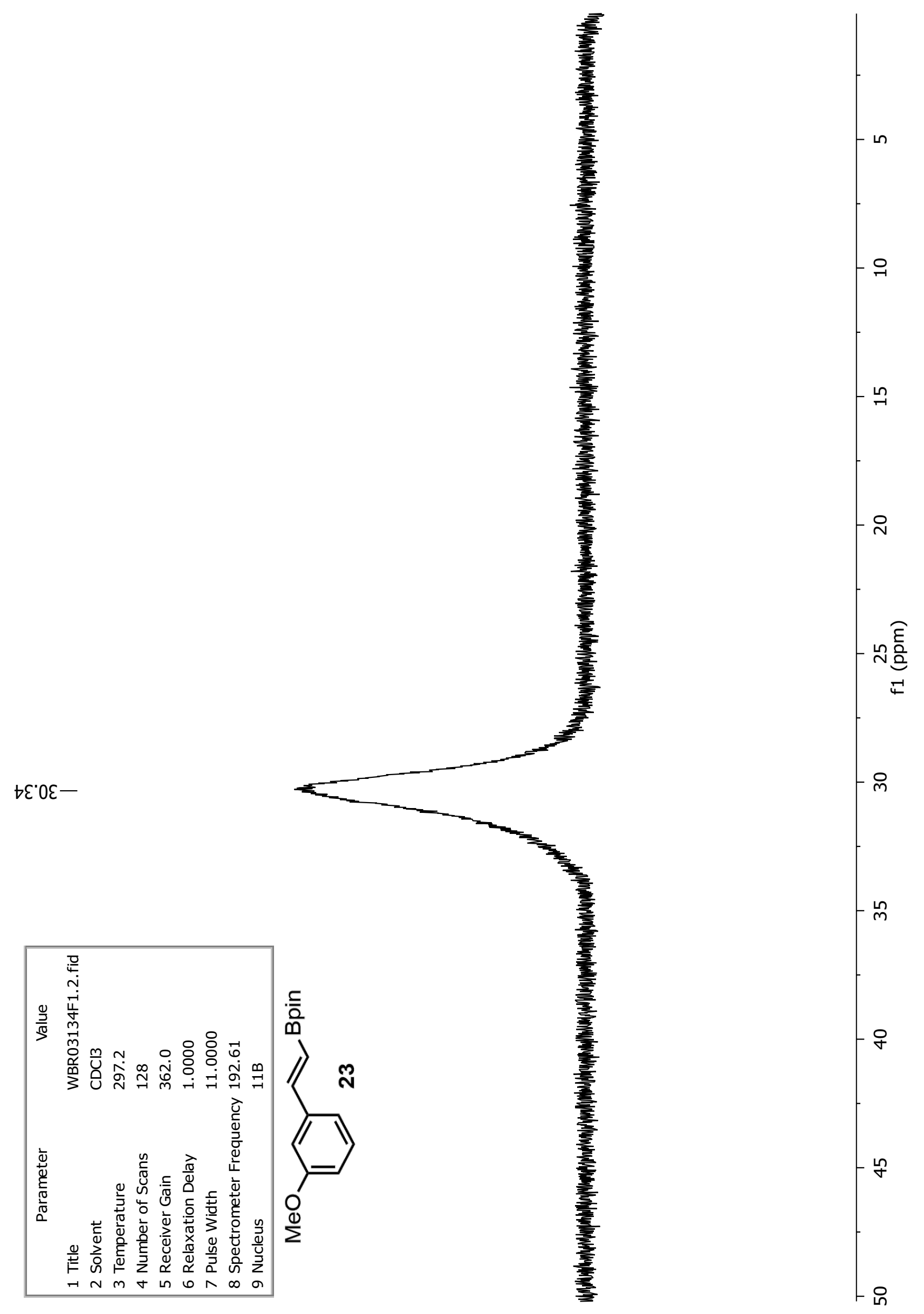



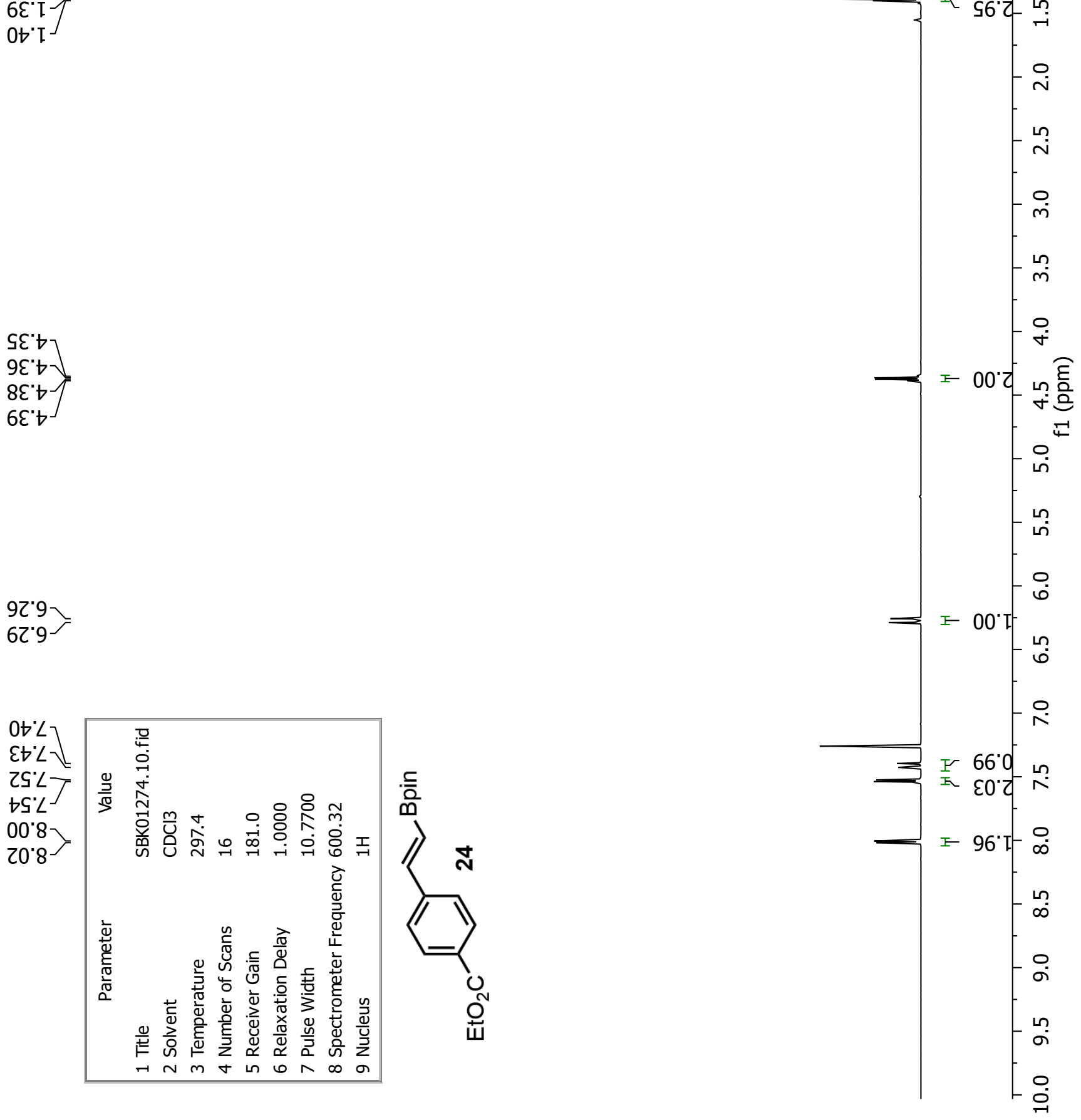
$8 t^{\circ} \sqcup I-$

$\angle 6^{\circ} \circ 2-$

2I'T9-

โL'\&8-

$00^{\circ} \angle Z I 乙$

Z0.0हI

S9.0Eโ $\checkmark$

$S \angle ' I D I-$
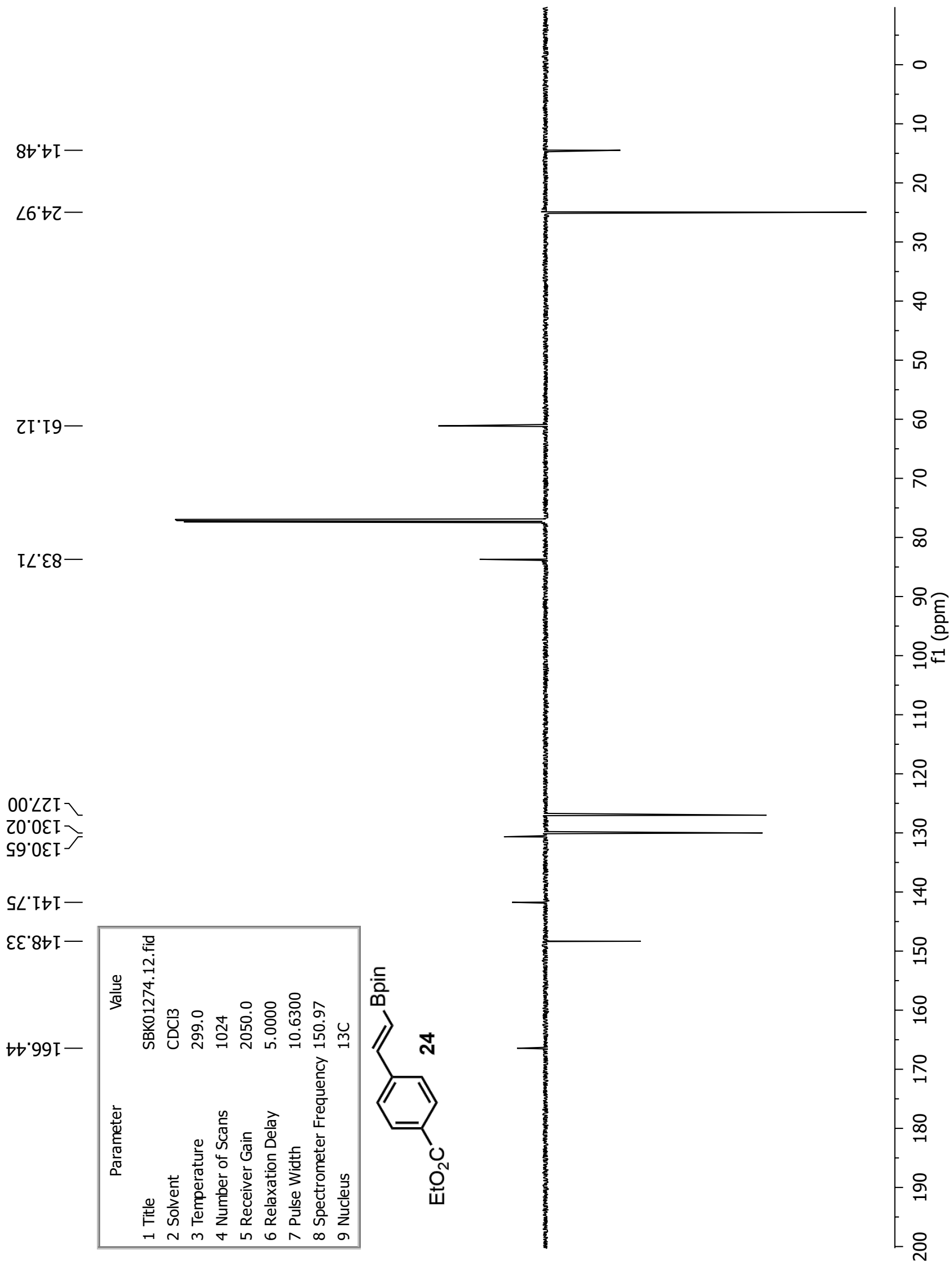


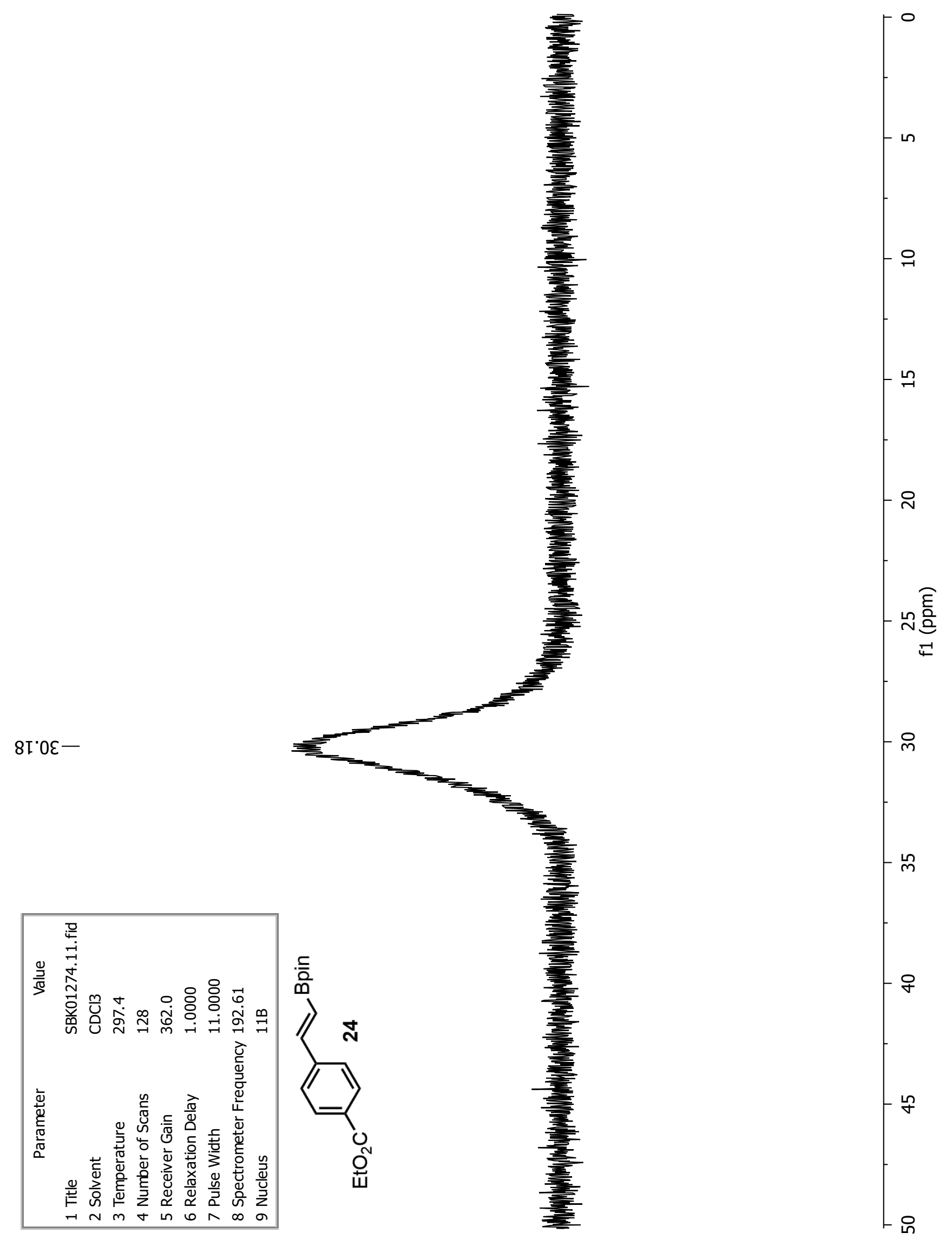




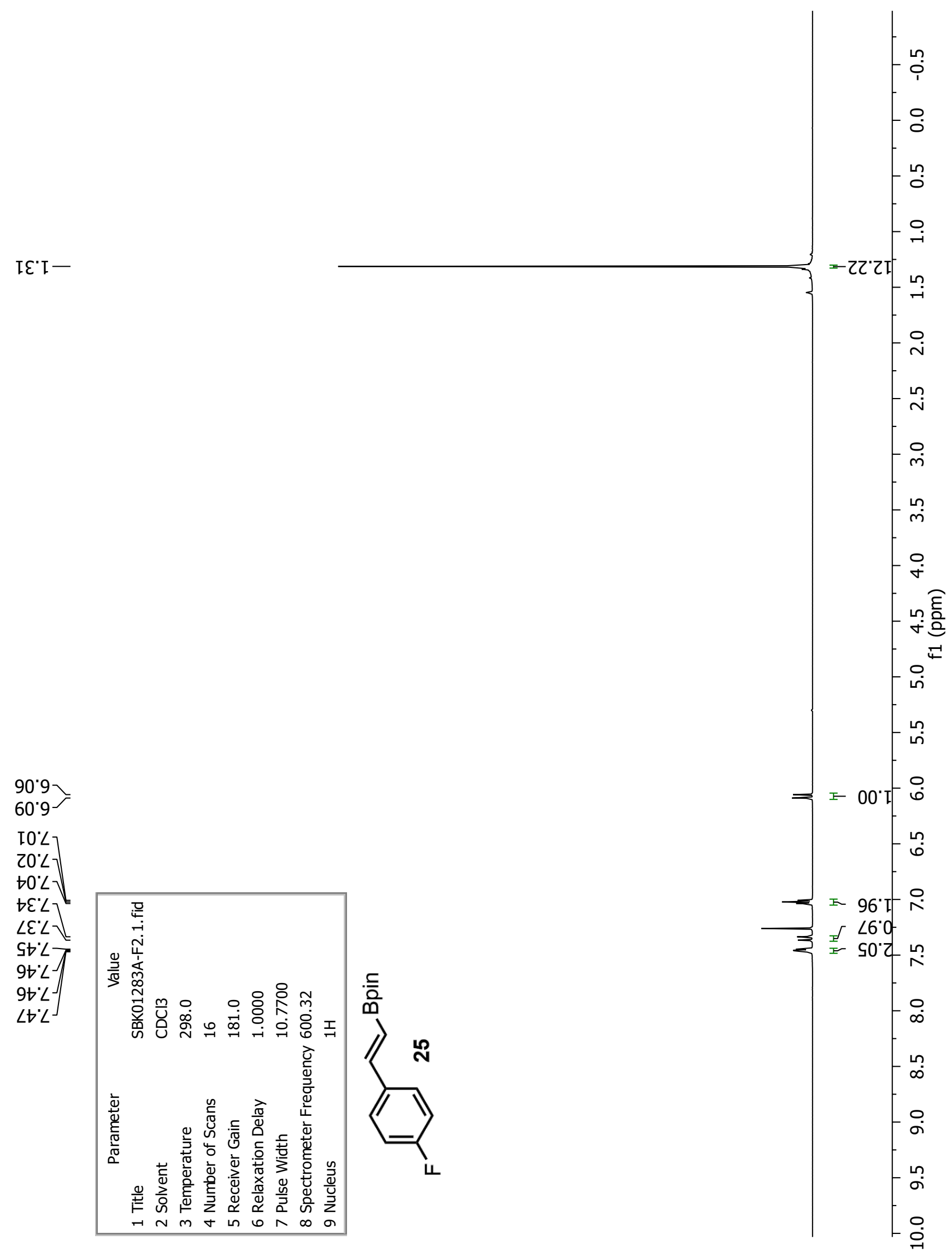


$56\llcorner z-$

${ } \varsigma^{\circ} \varepsilon 8-$

6S'SII

$08^{\circ} 82 \mathrm{~T}$

88.8ZI

โ8'દEโ

S8'દદโ

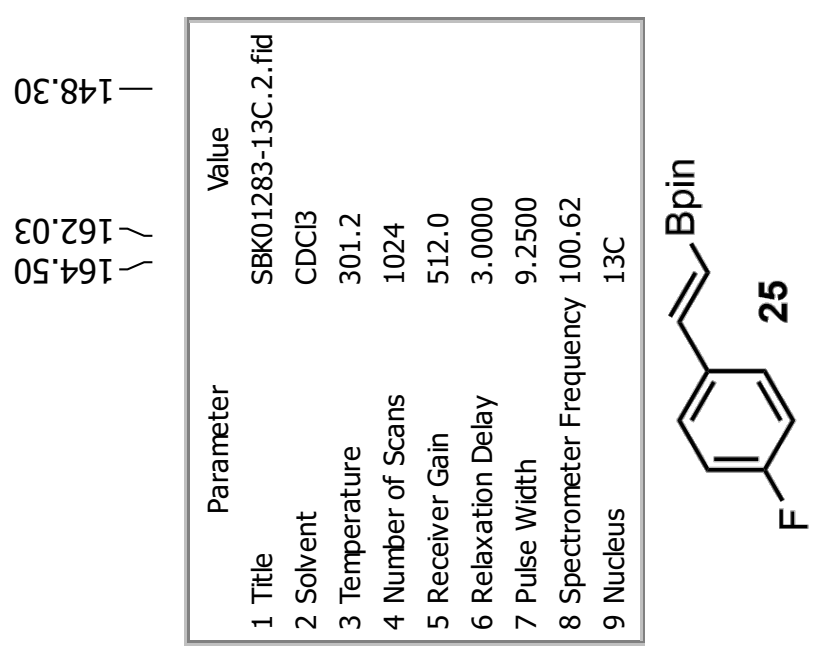

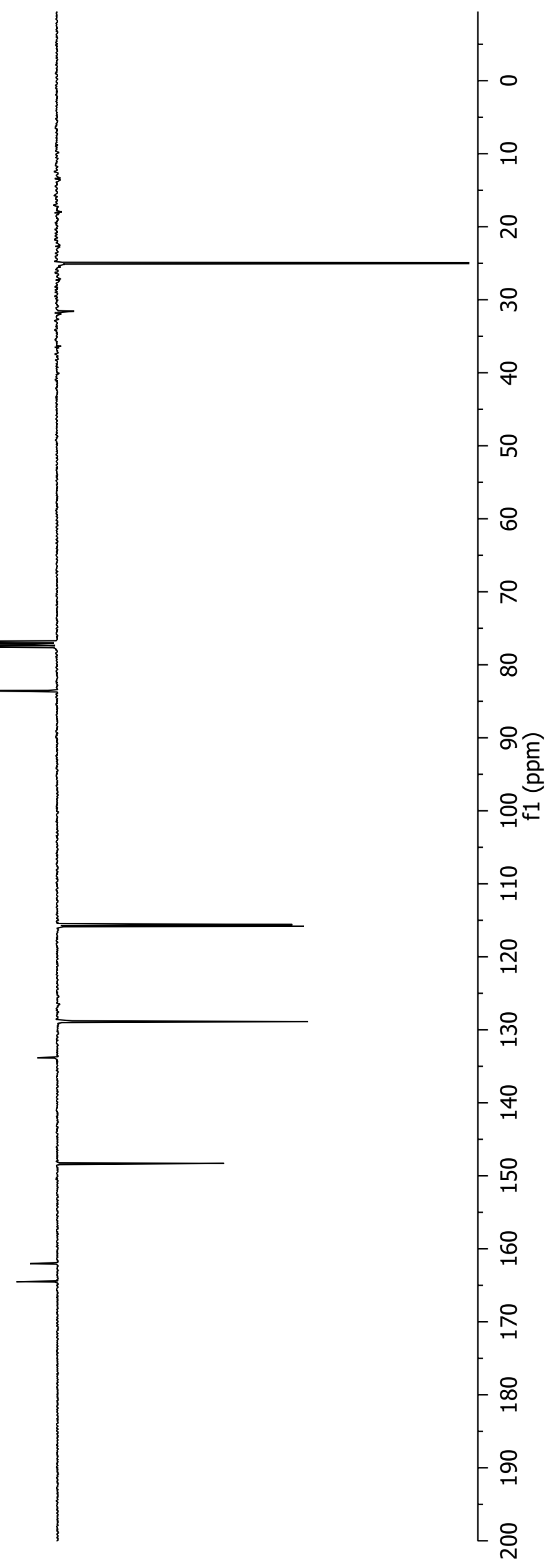




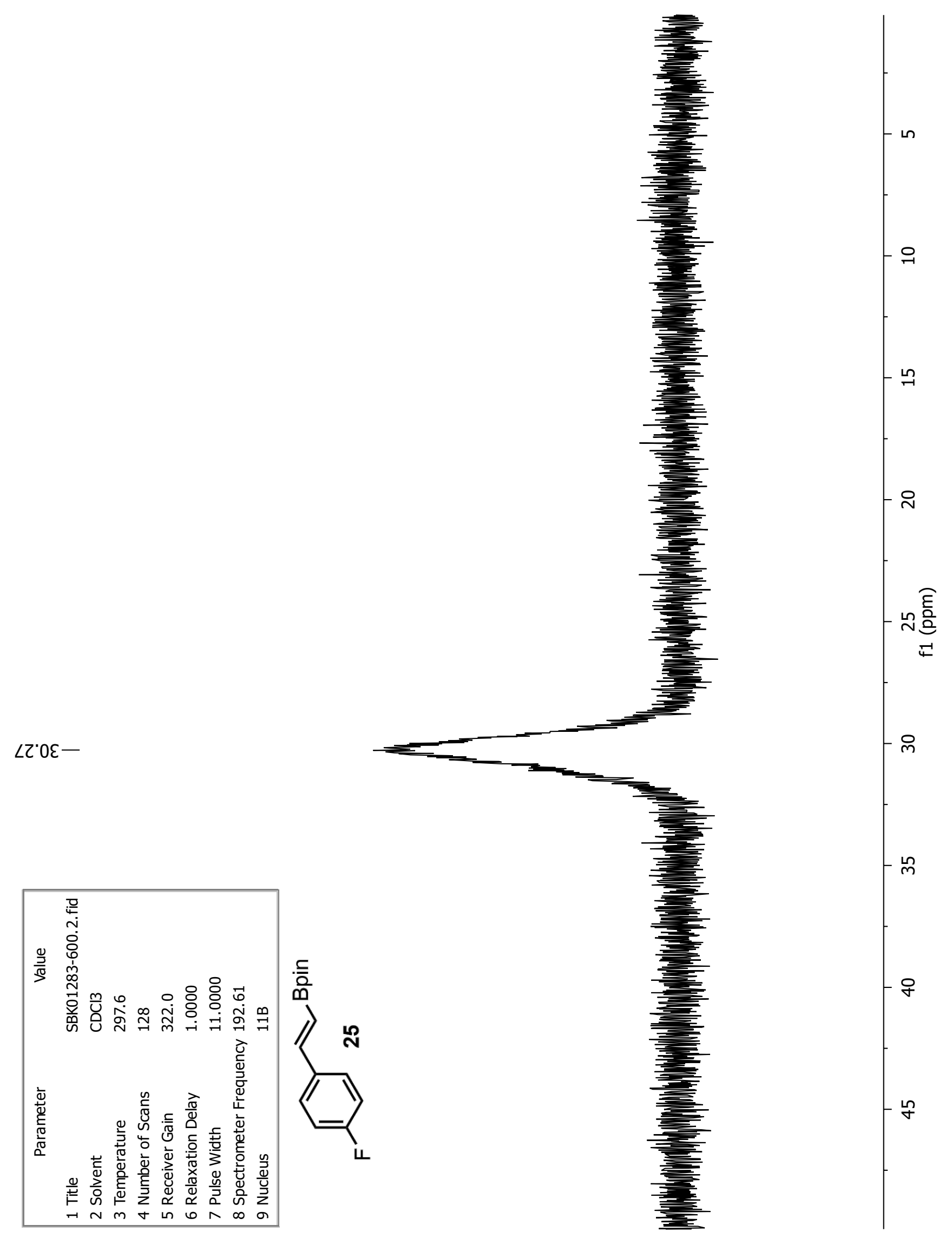




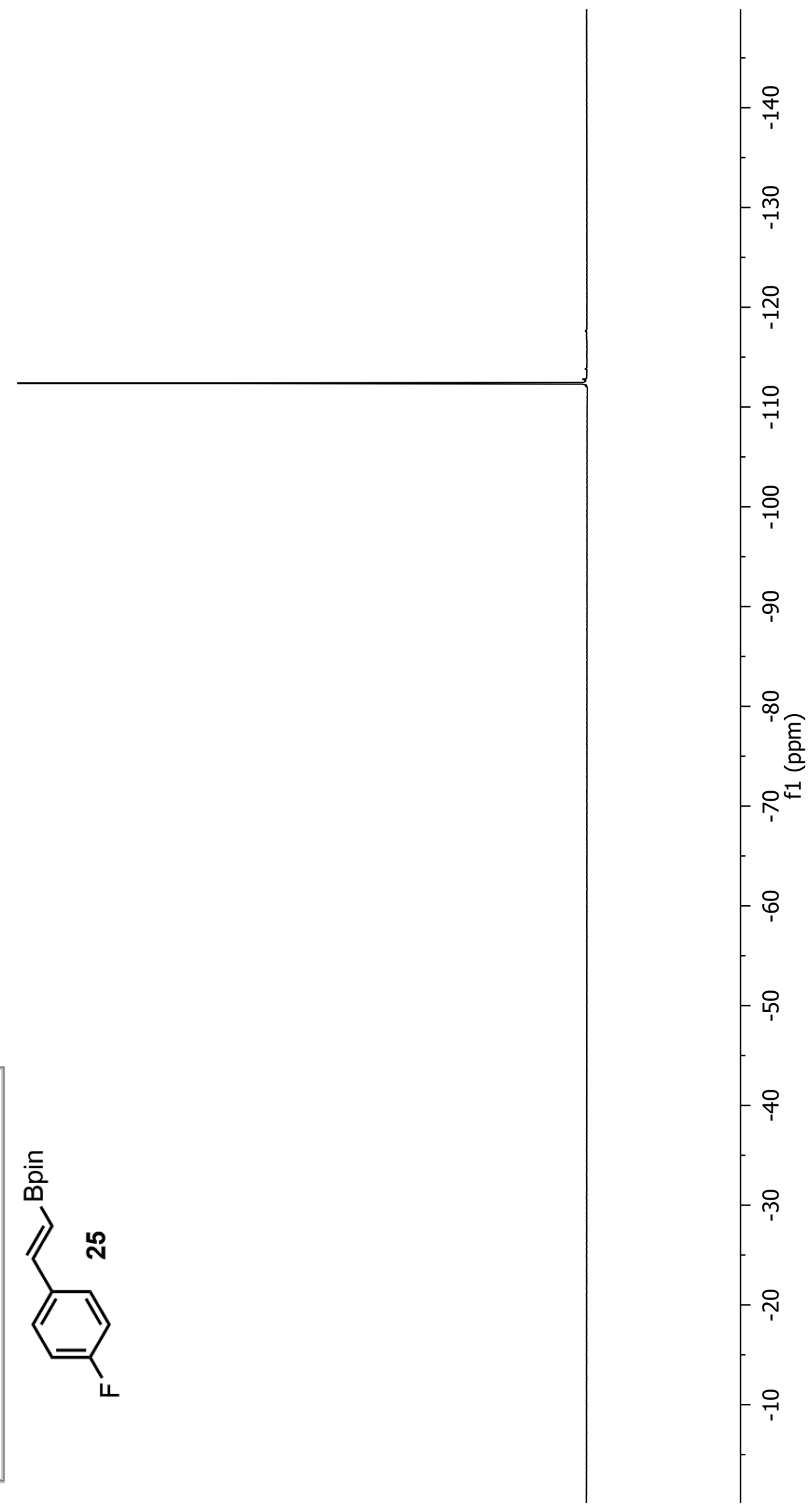




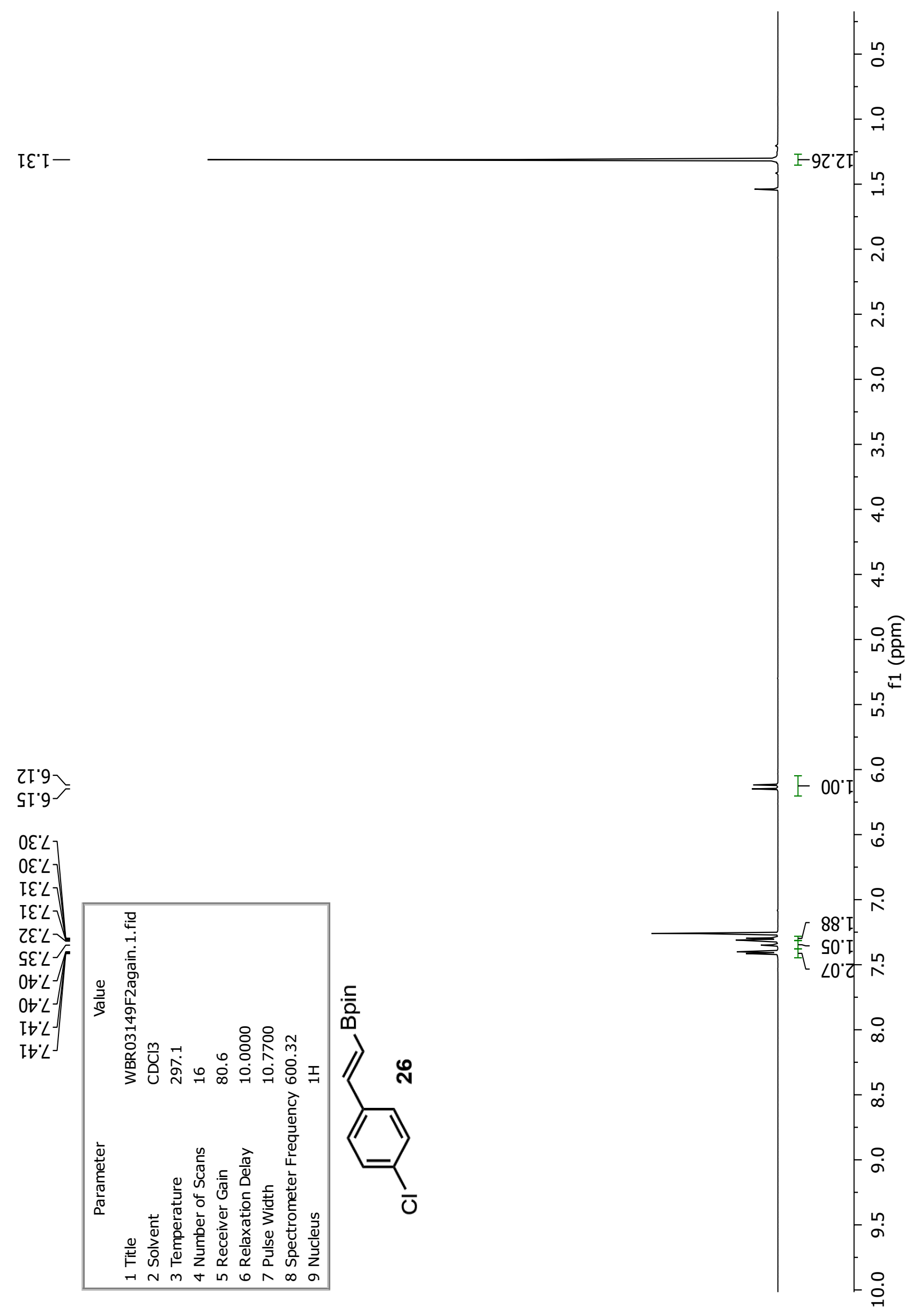




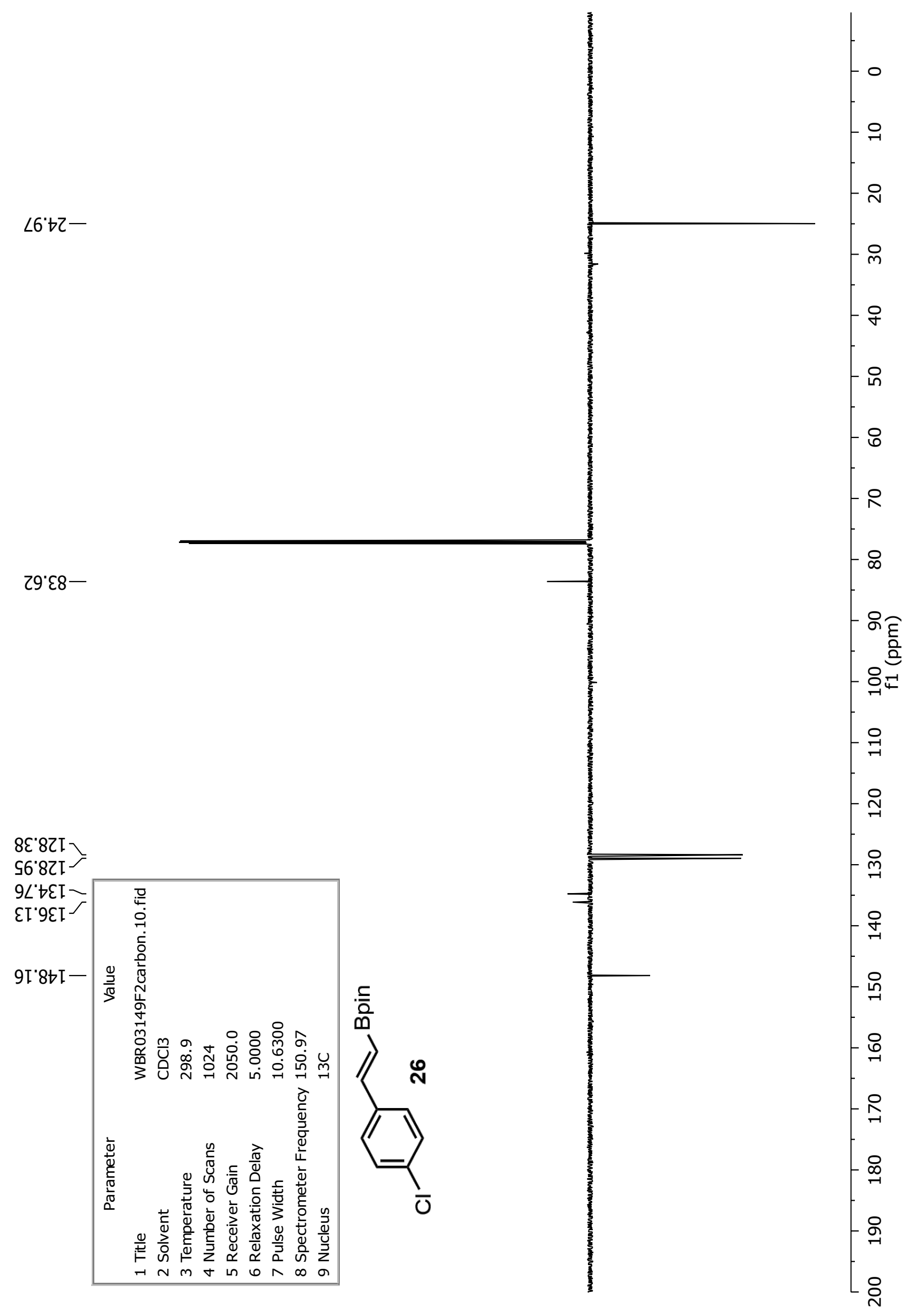




$$
\}
$$




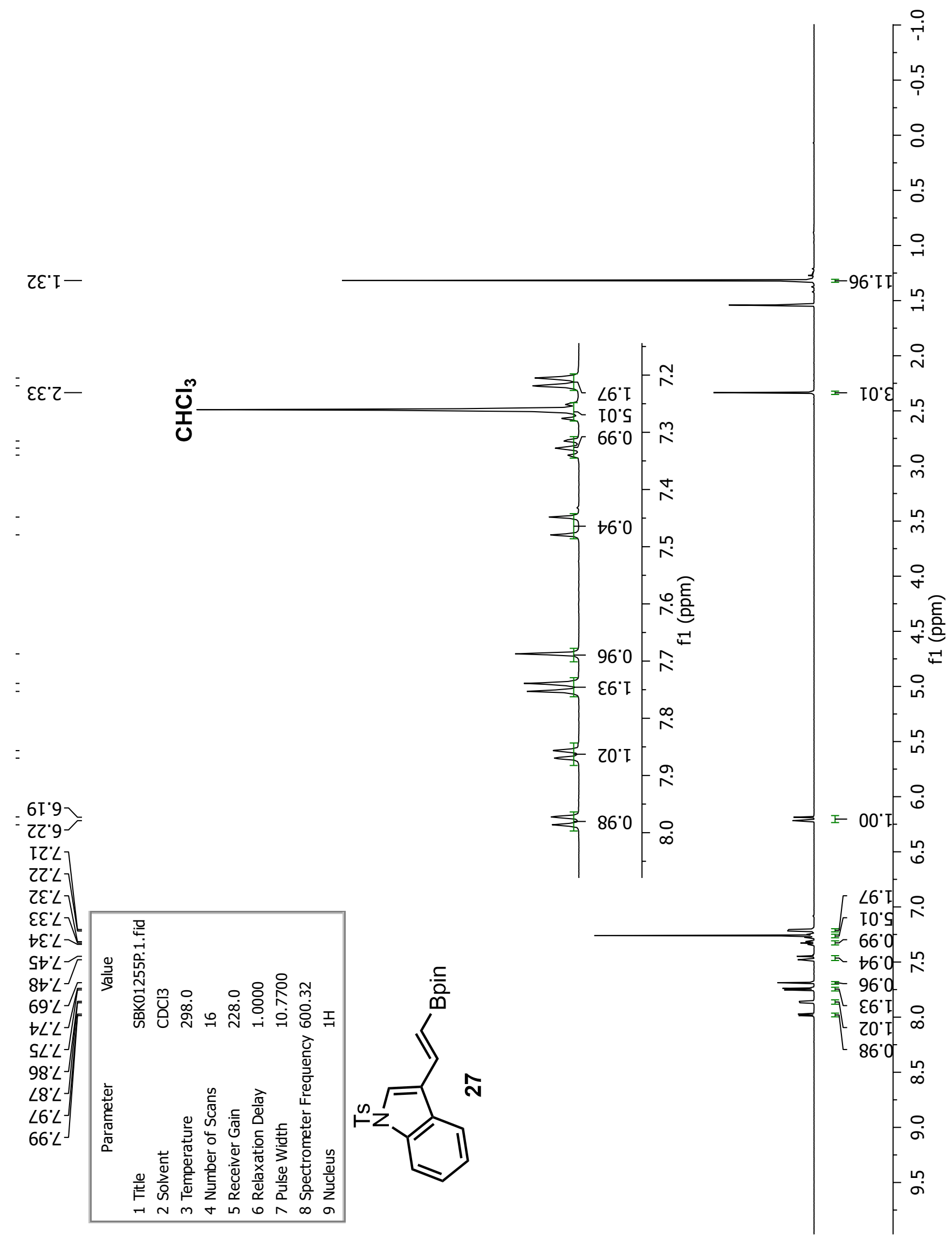




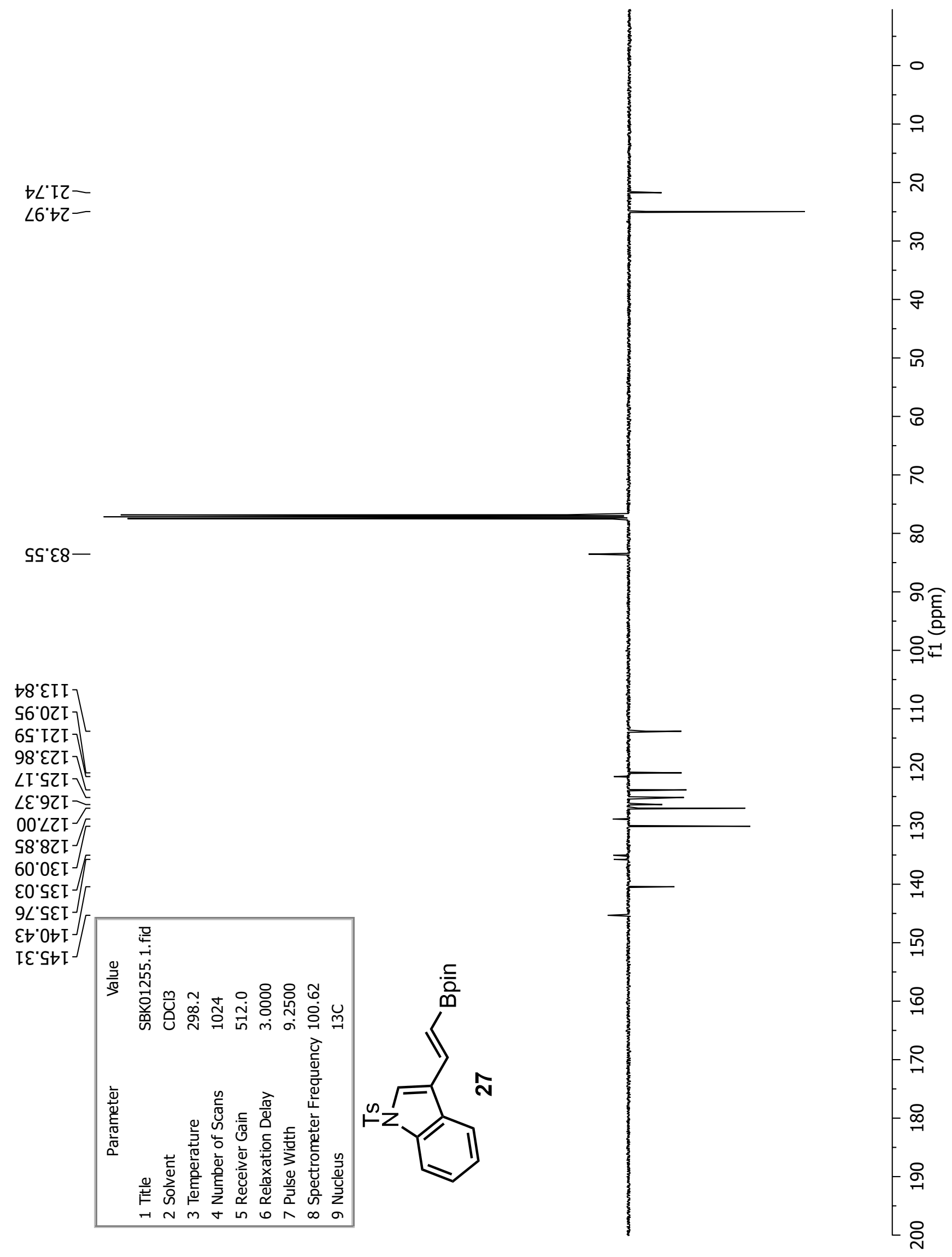



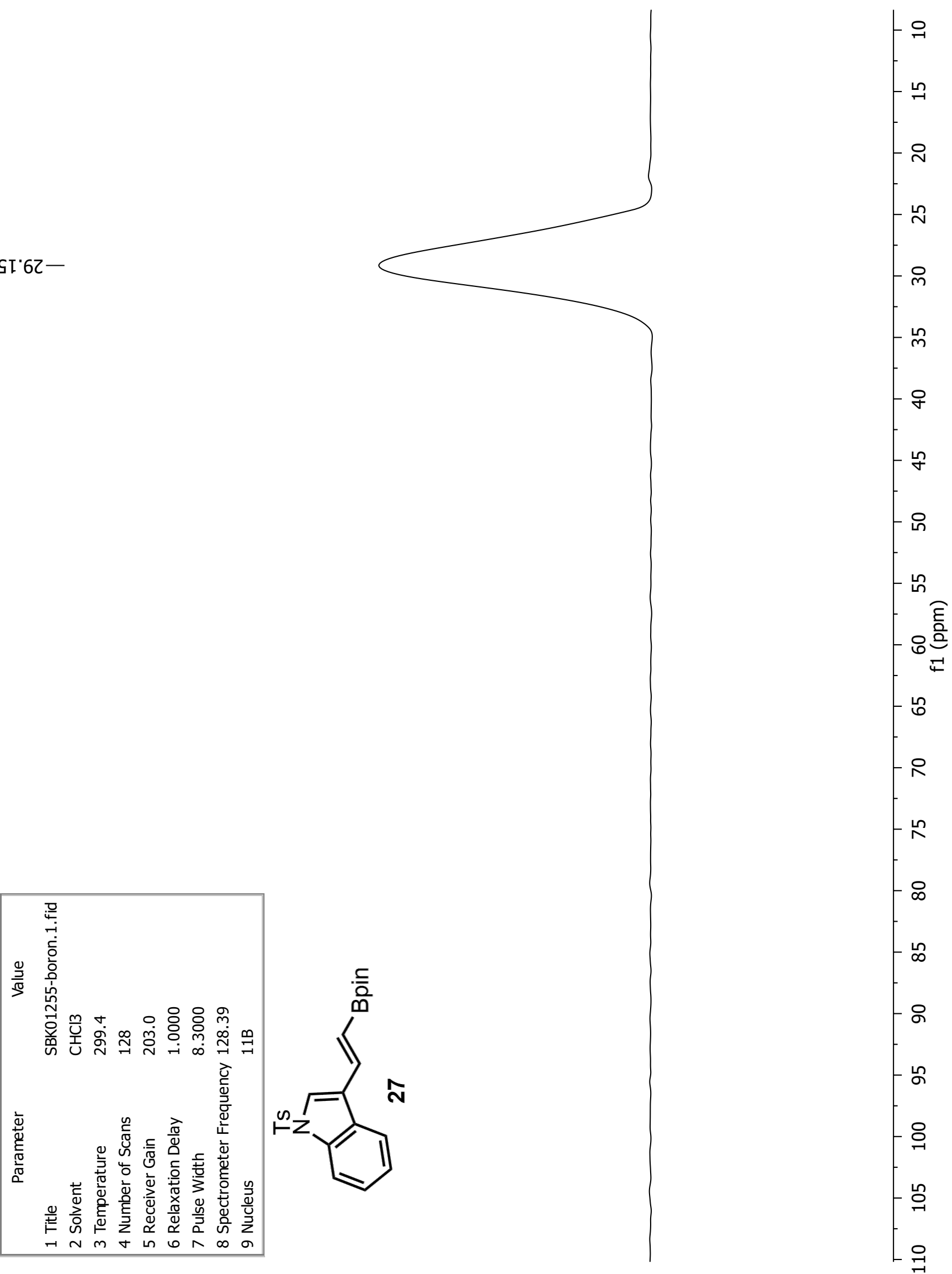
IE' I-
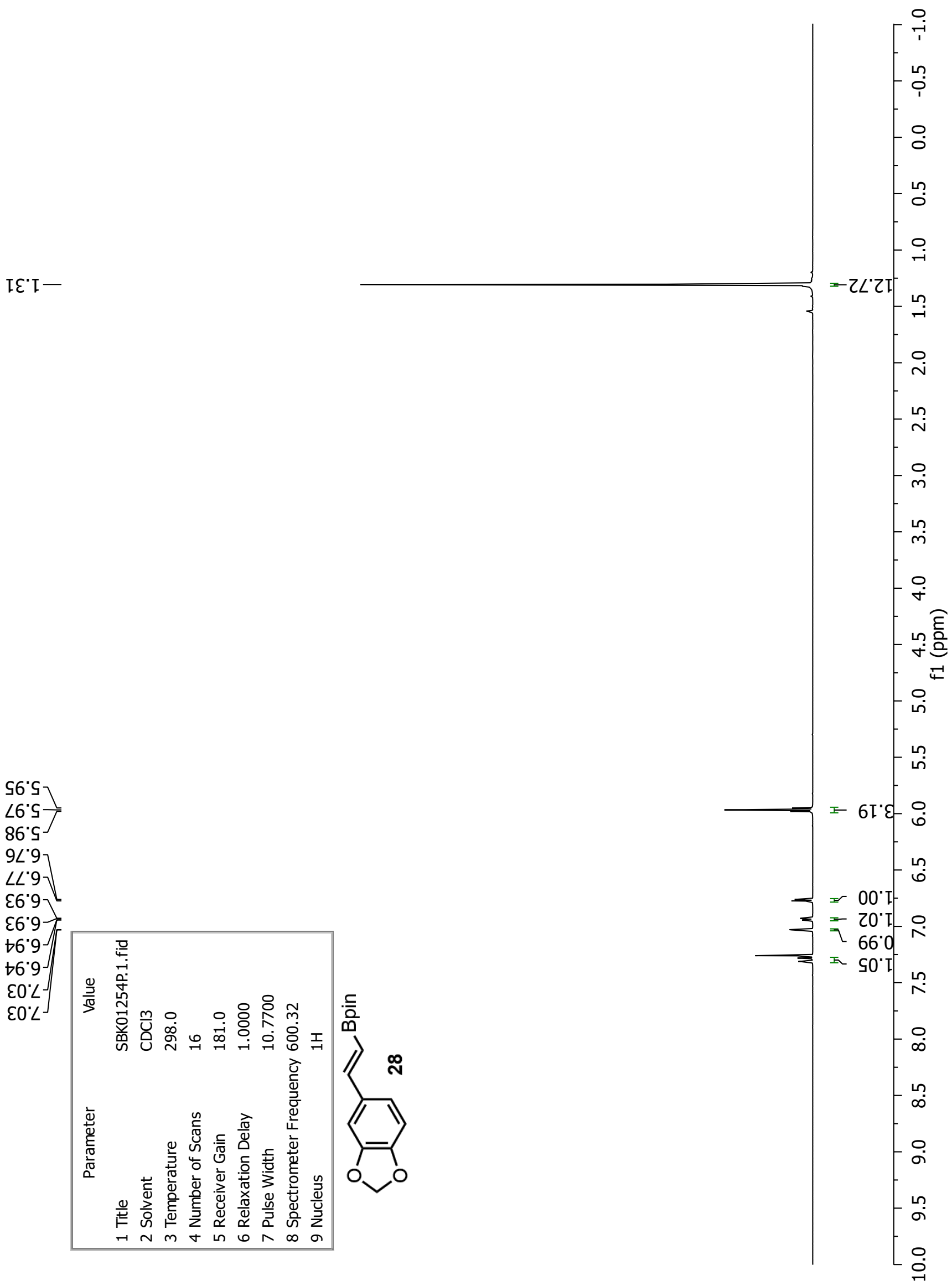
$96^{\circ} \circ z-$

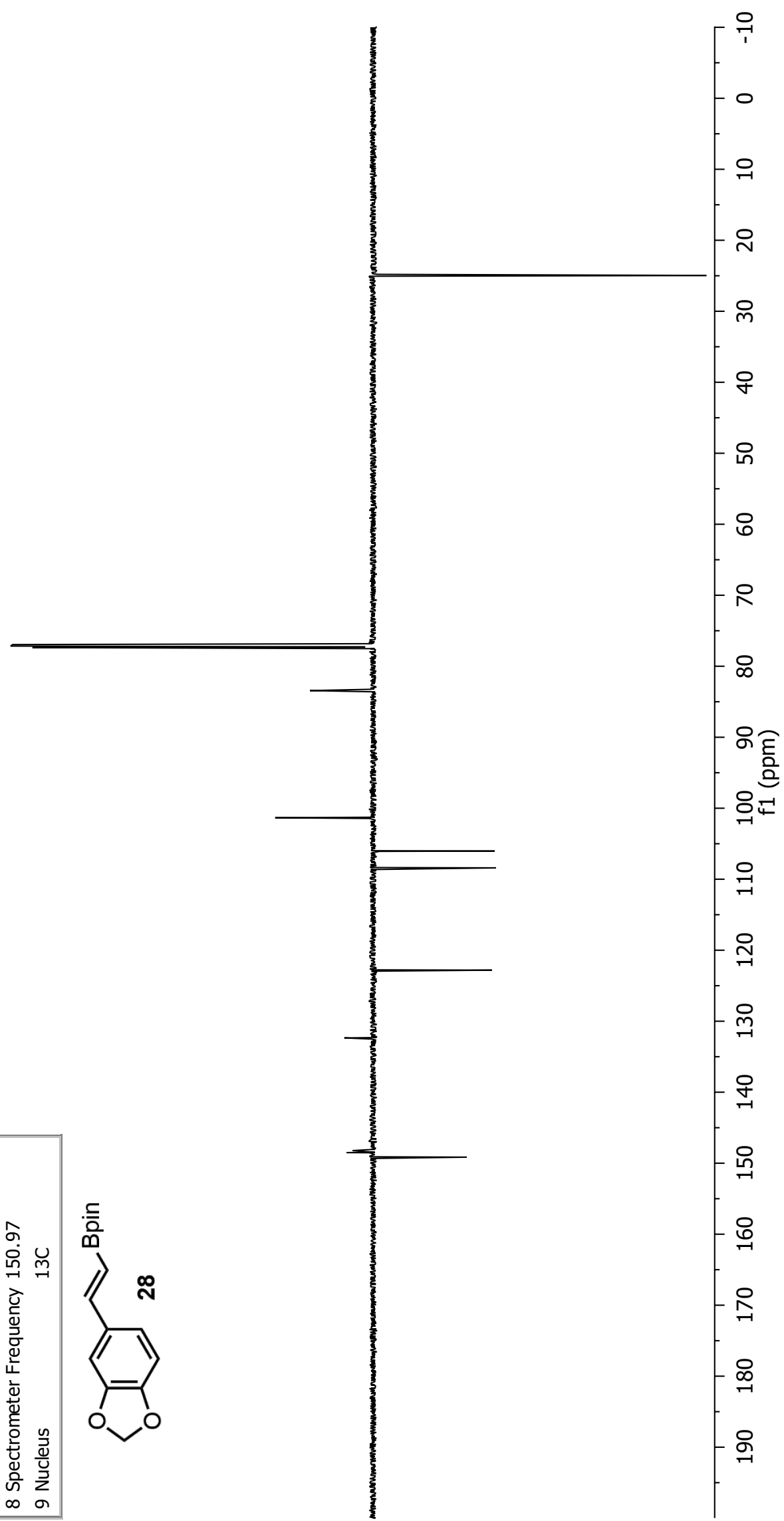

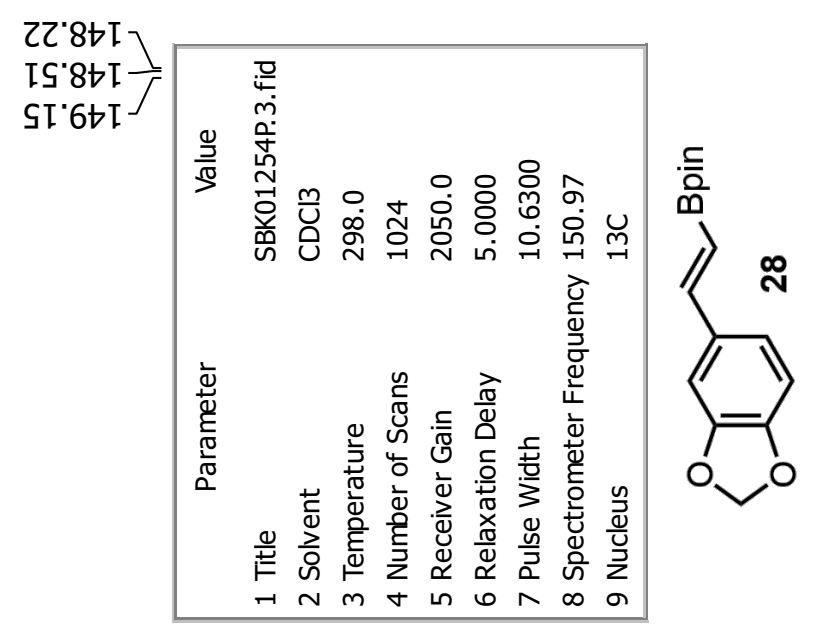

tE. TOL -

$\rightarrow 0.90 \mathrm{I} 工$

$0 t^{\circ} 80$ I -

โ8'ว2I-

ธદ'乙عI-

우

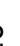

ع๑. ع8- 


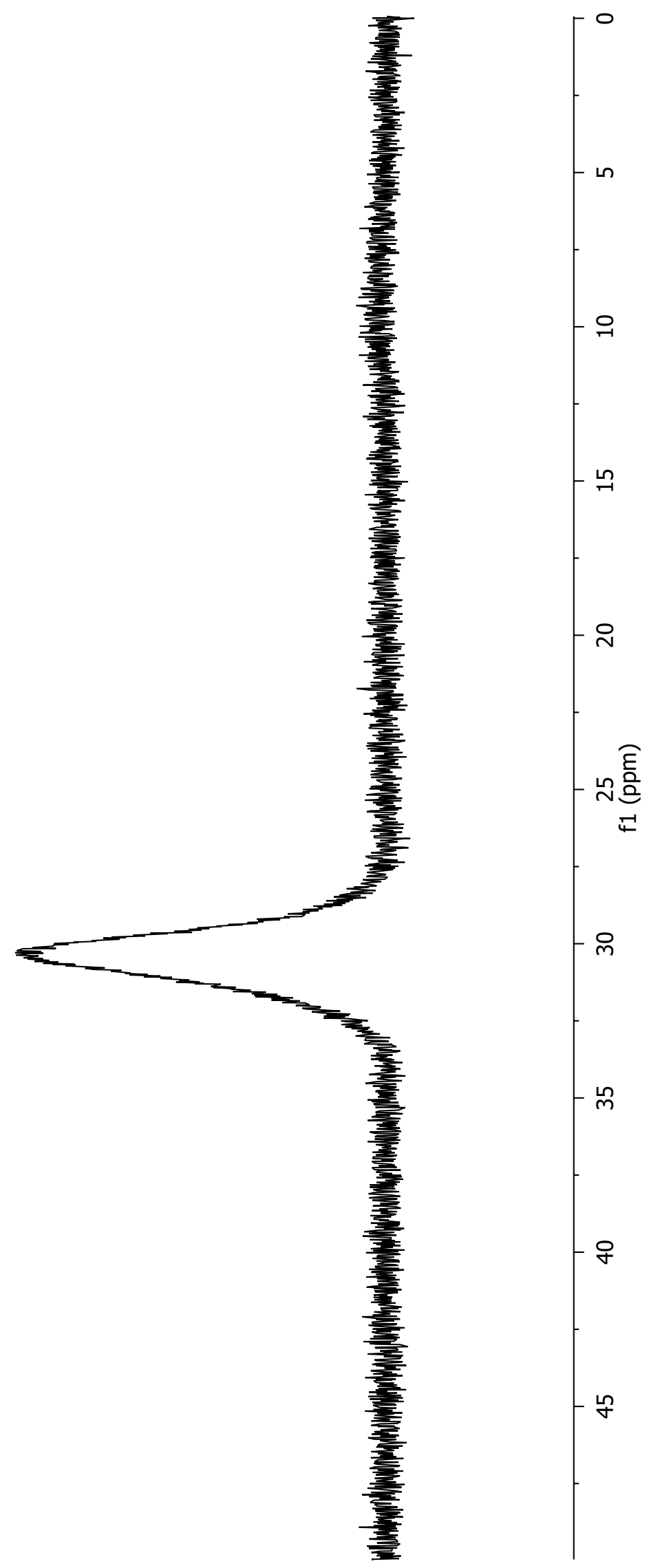




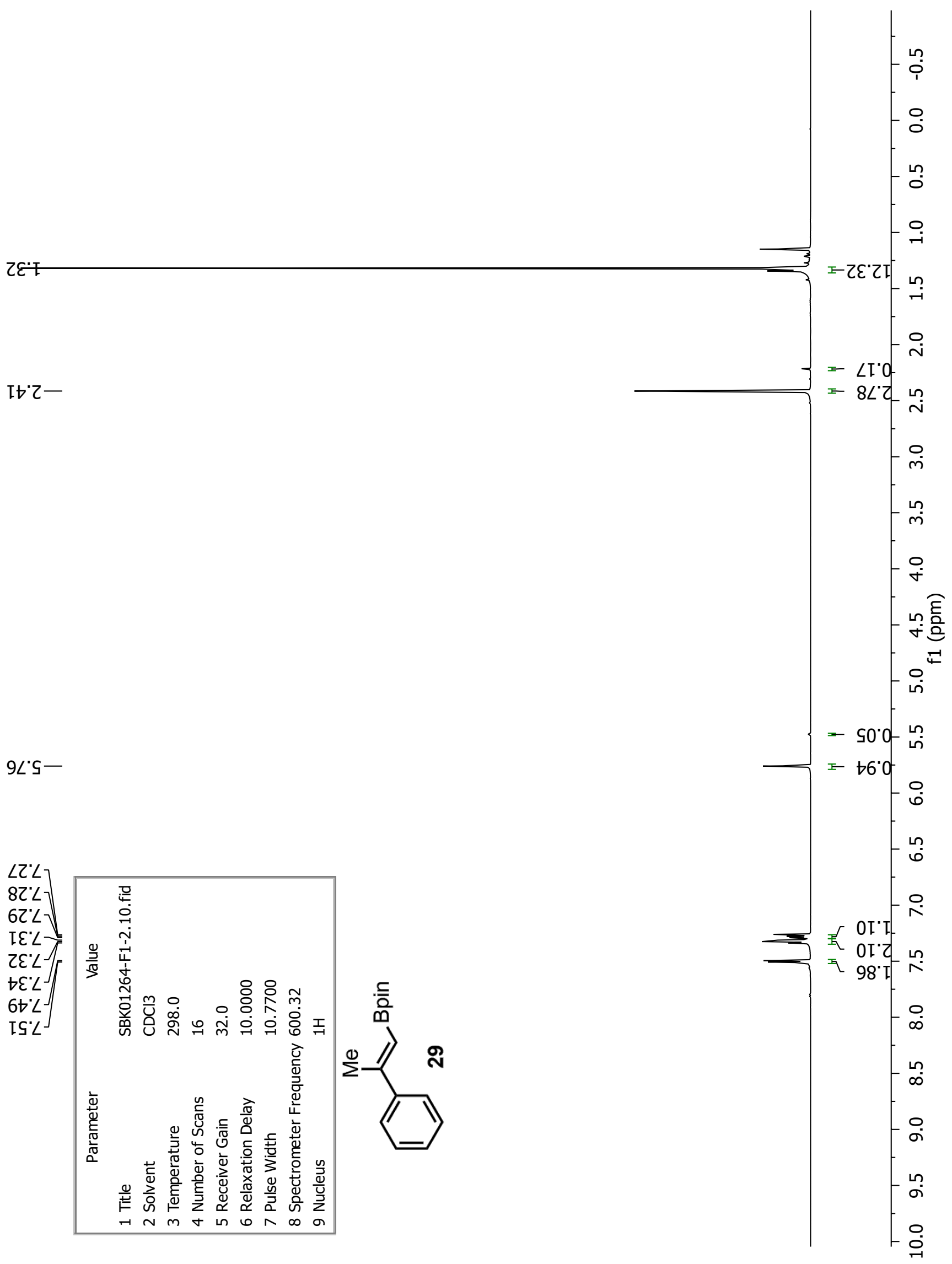




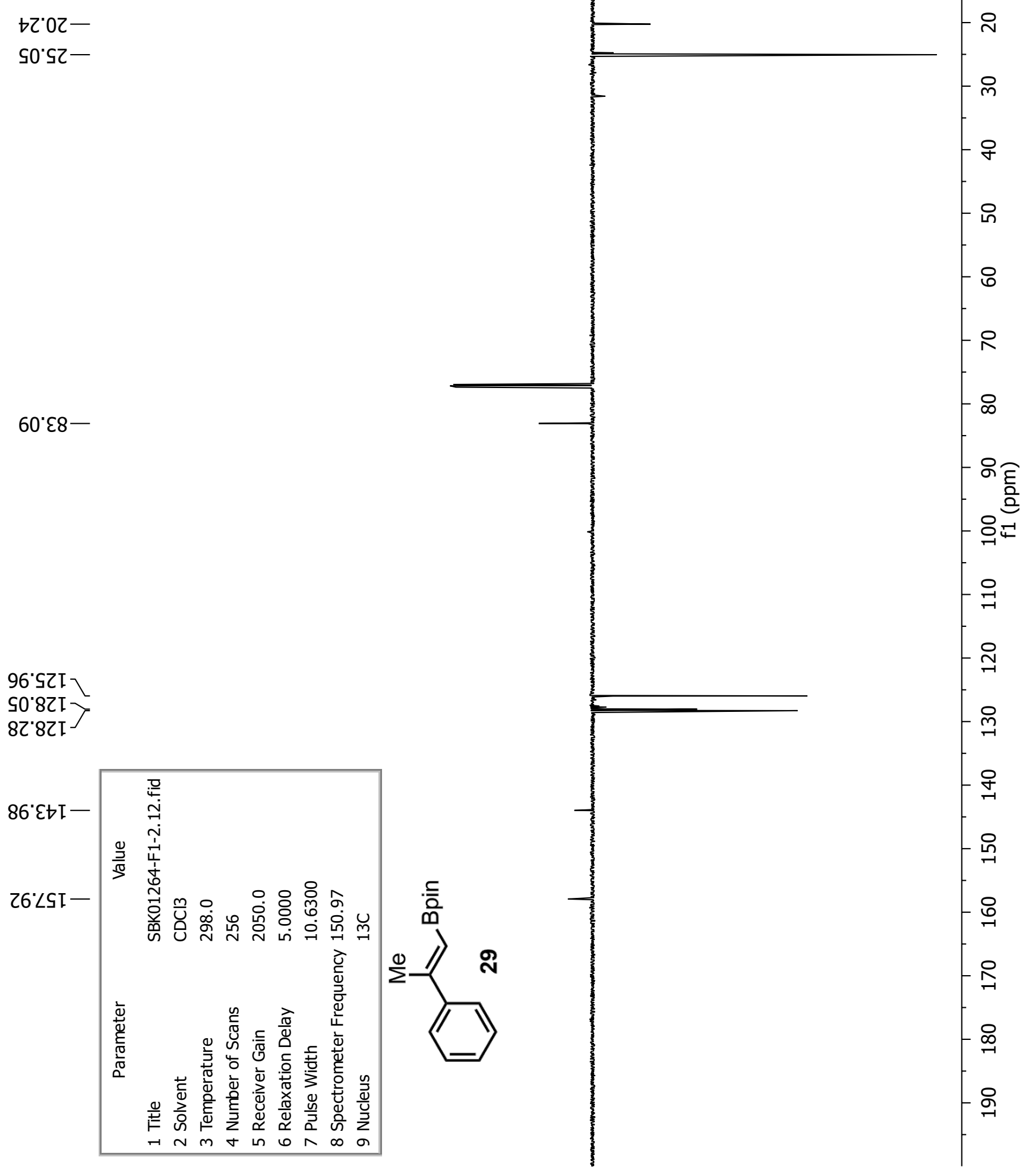




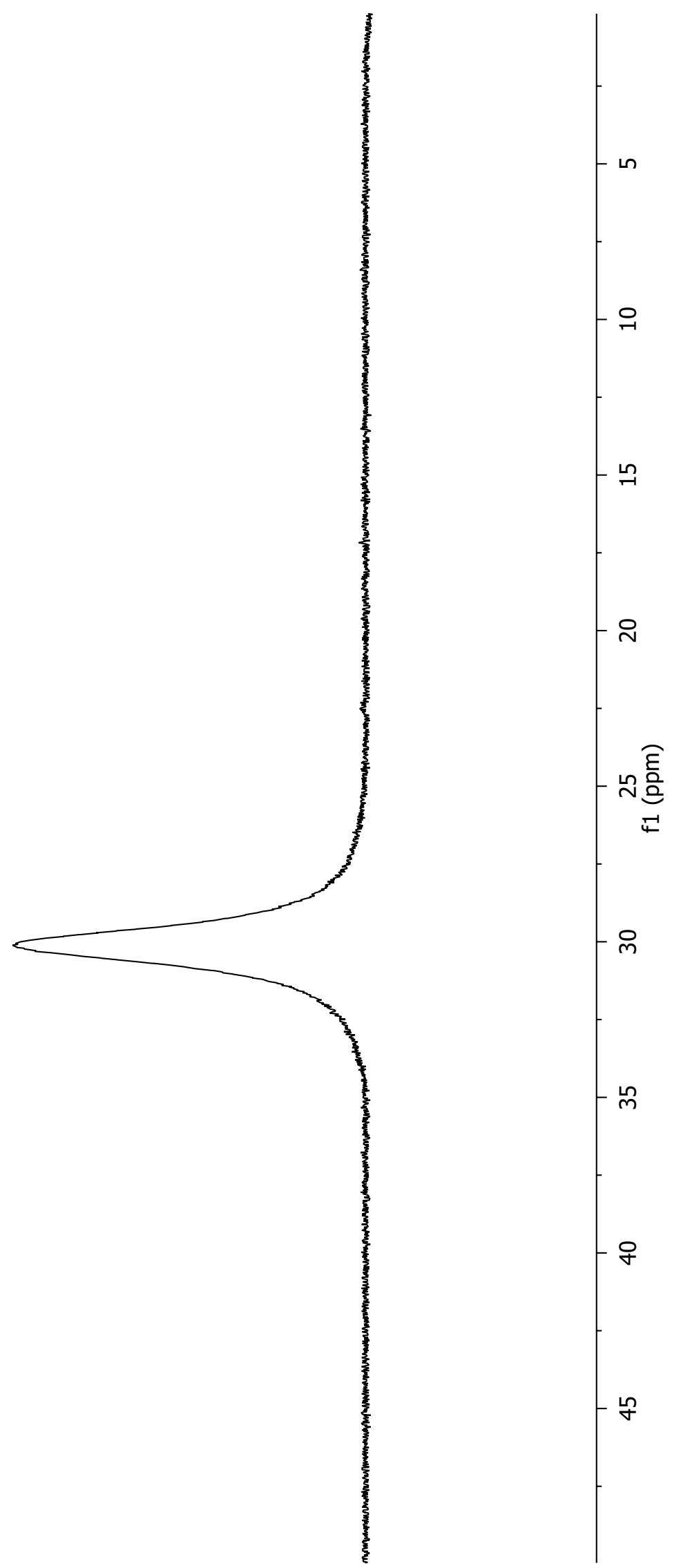




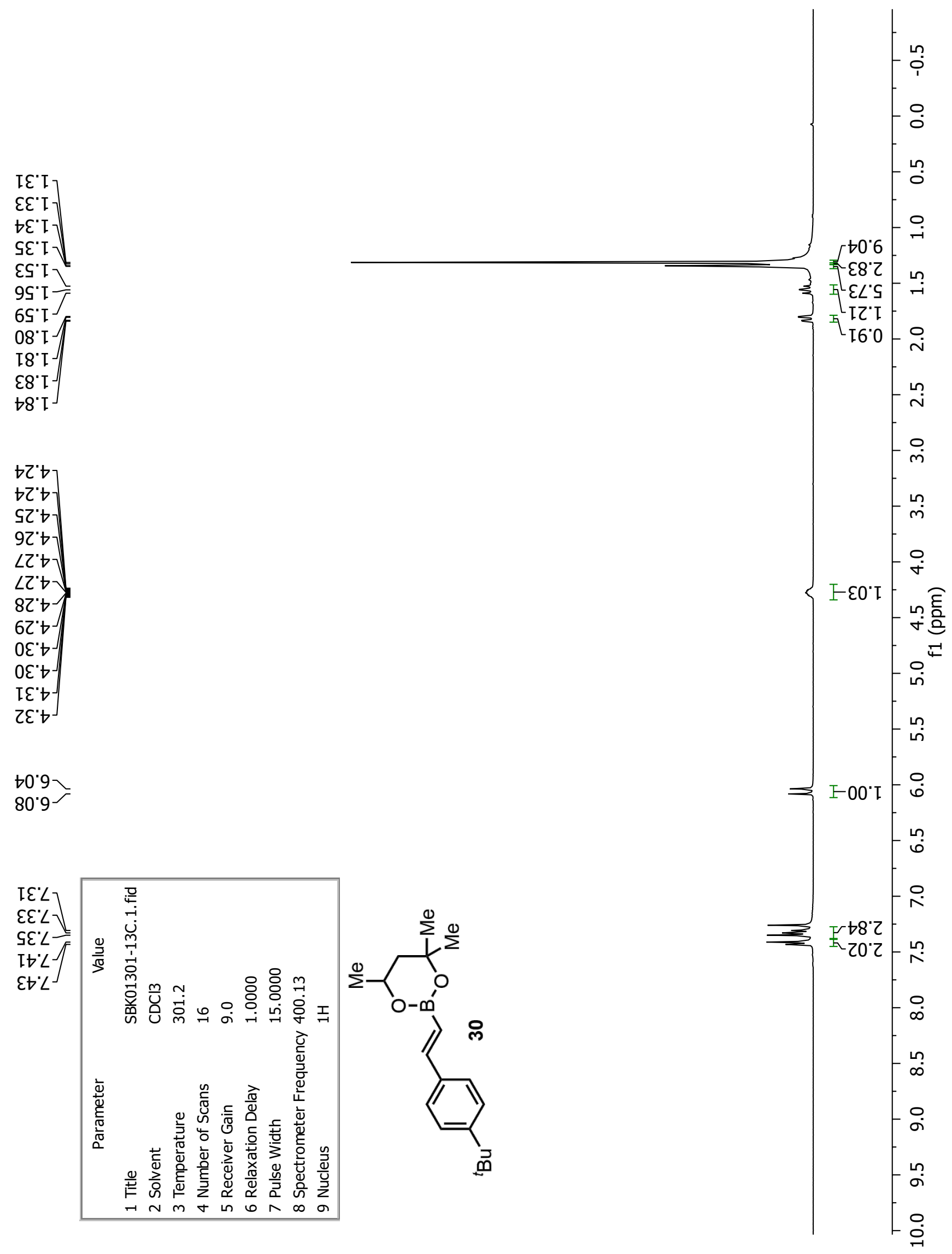


८ย'ย乙-

乙ย'8乙

乙ち. โE-

$8 L^{\prime} \downarrow \varepsilon-$

$\downarrow I^{\circ} 9 b-$

$56.59-$

$86^{\circ} 0 \angle-$

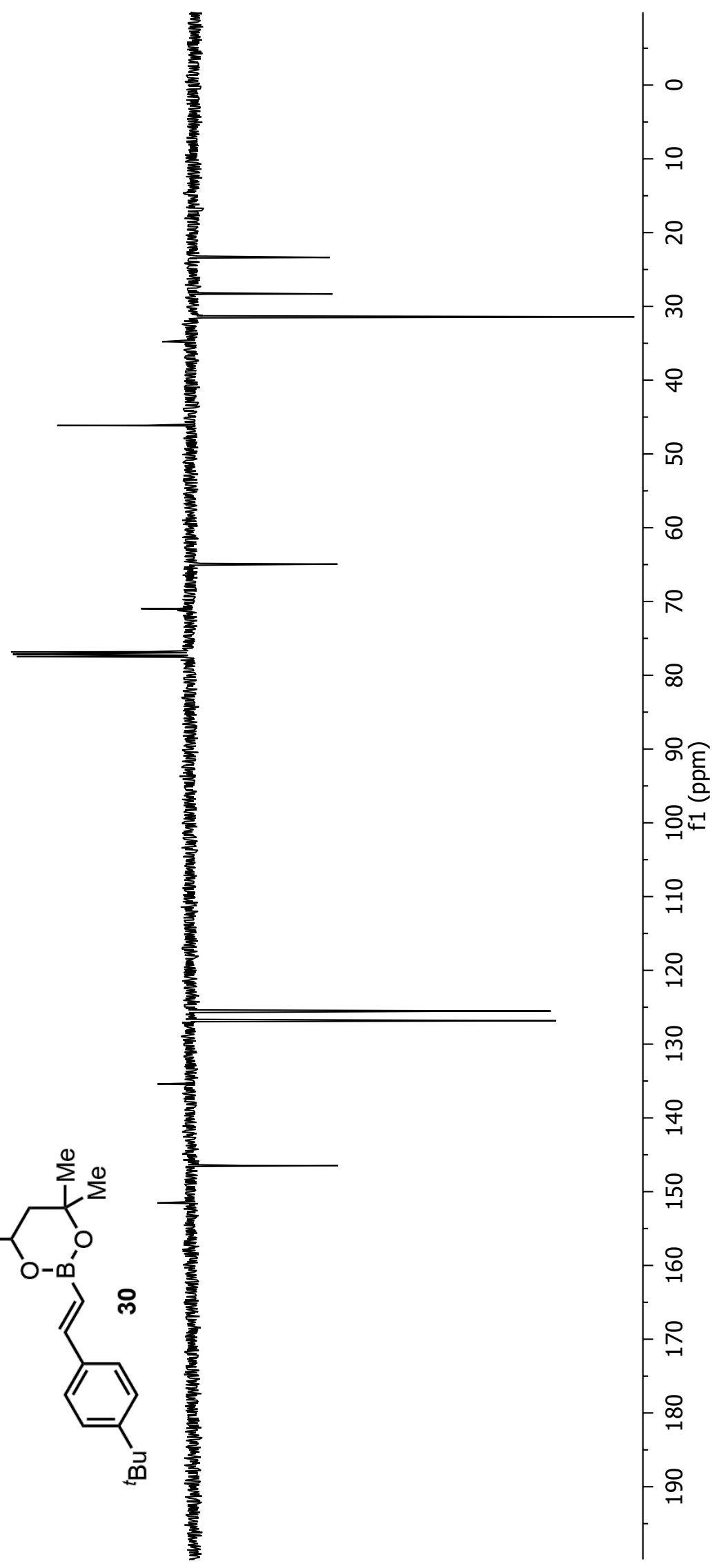

ZS'SZI

ह8.9ZI

I†'SEI -

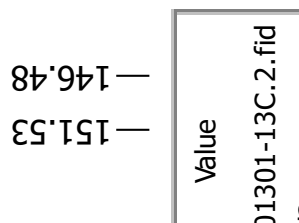

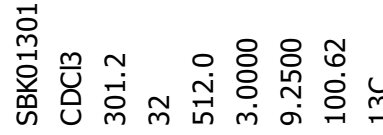

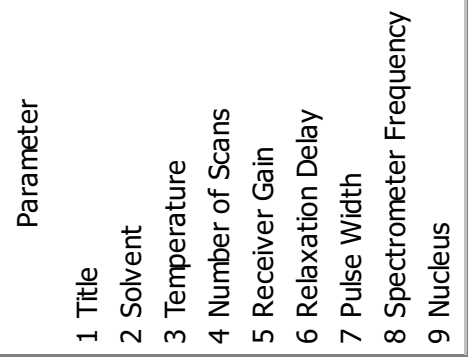



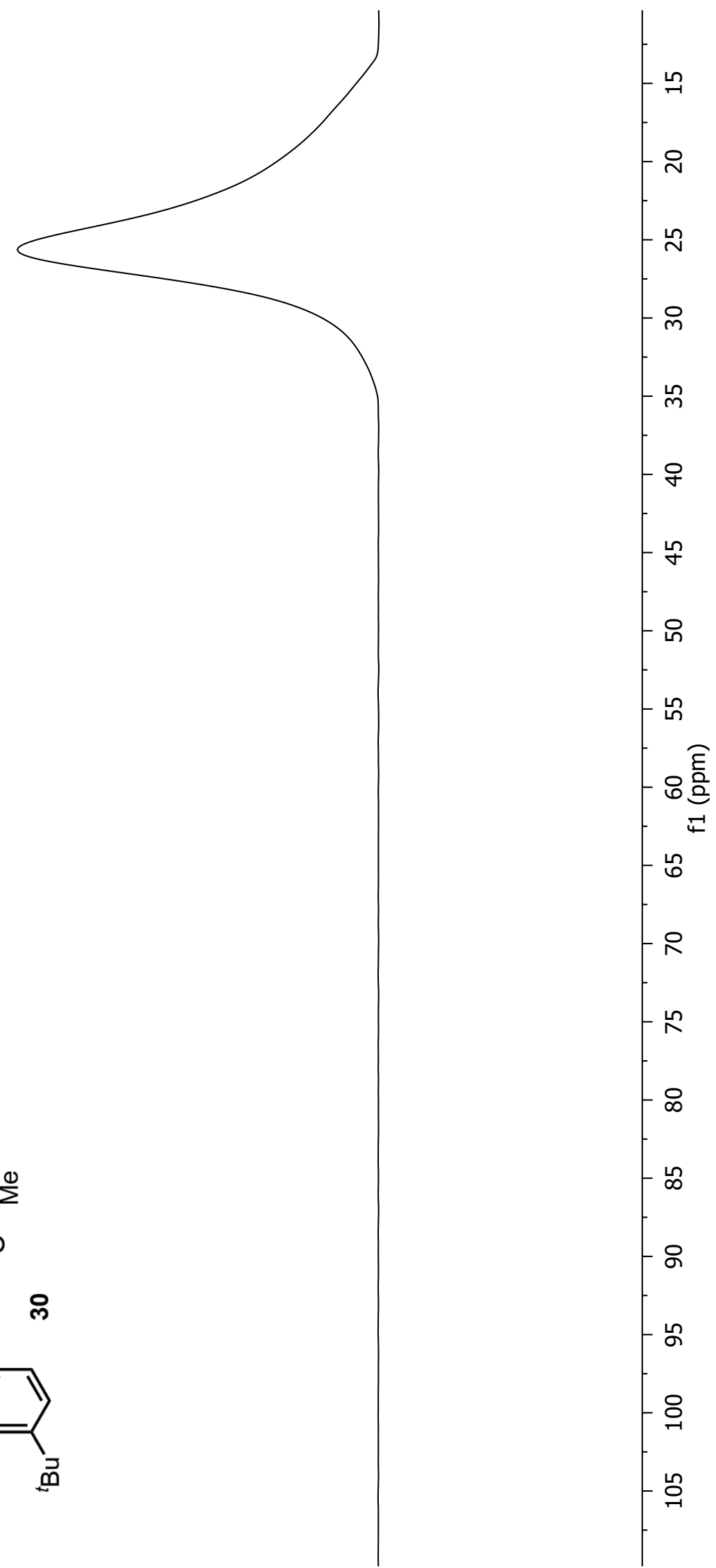
I0' I -

โE' I-

$0 L^{\circ} \varepsilon-$

t0.9

$\angle 0^{\circ} 9^{-}$

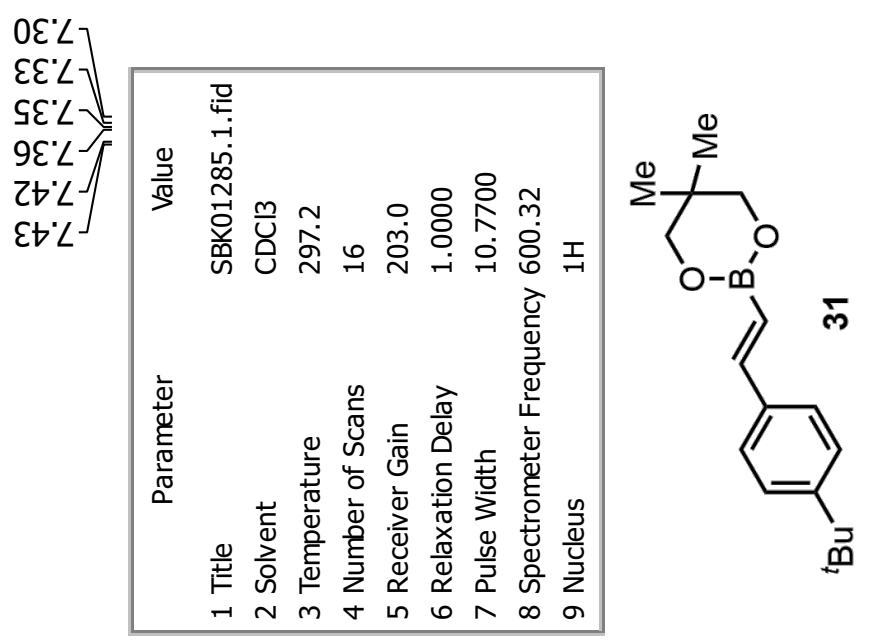

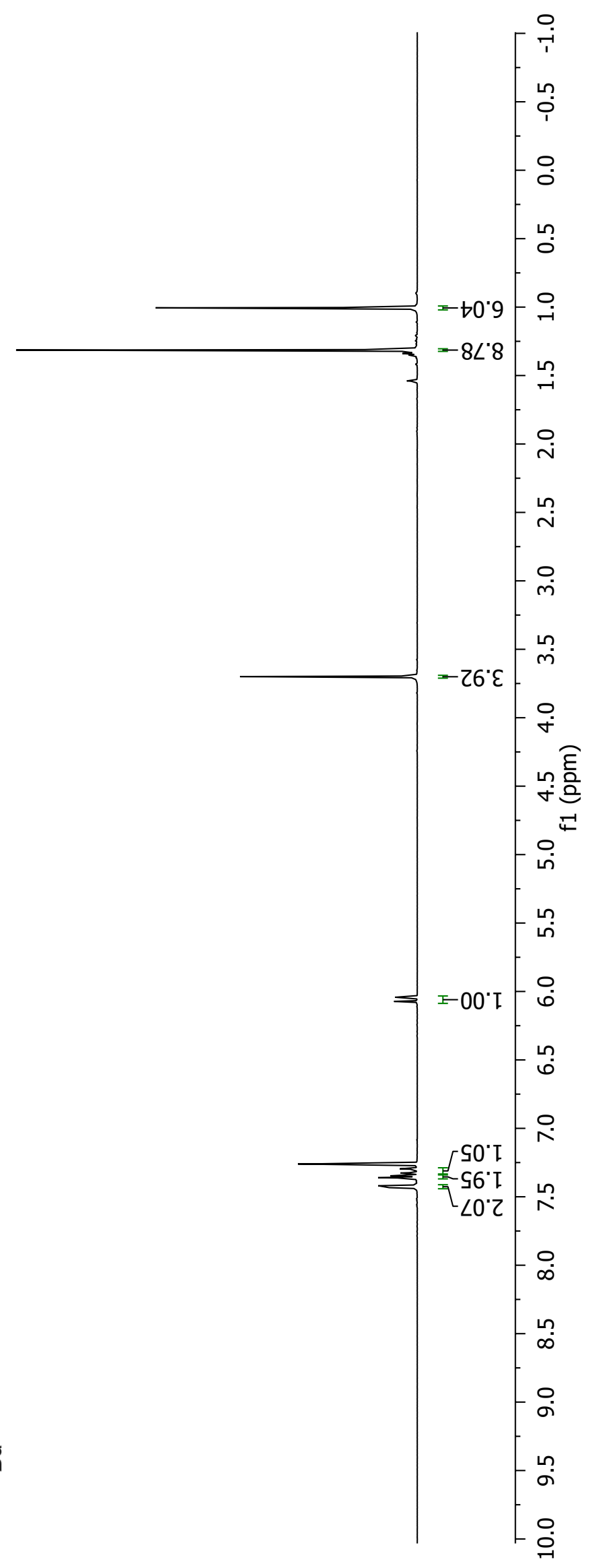




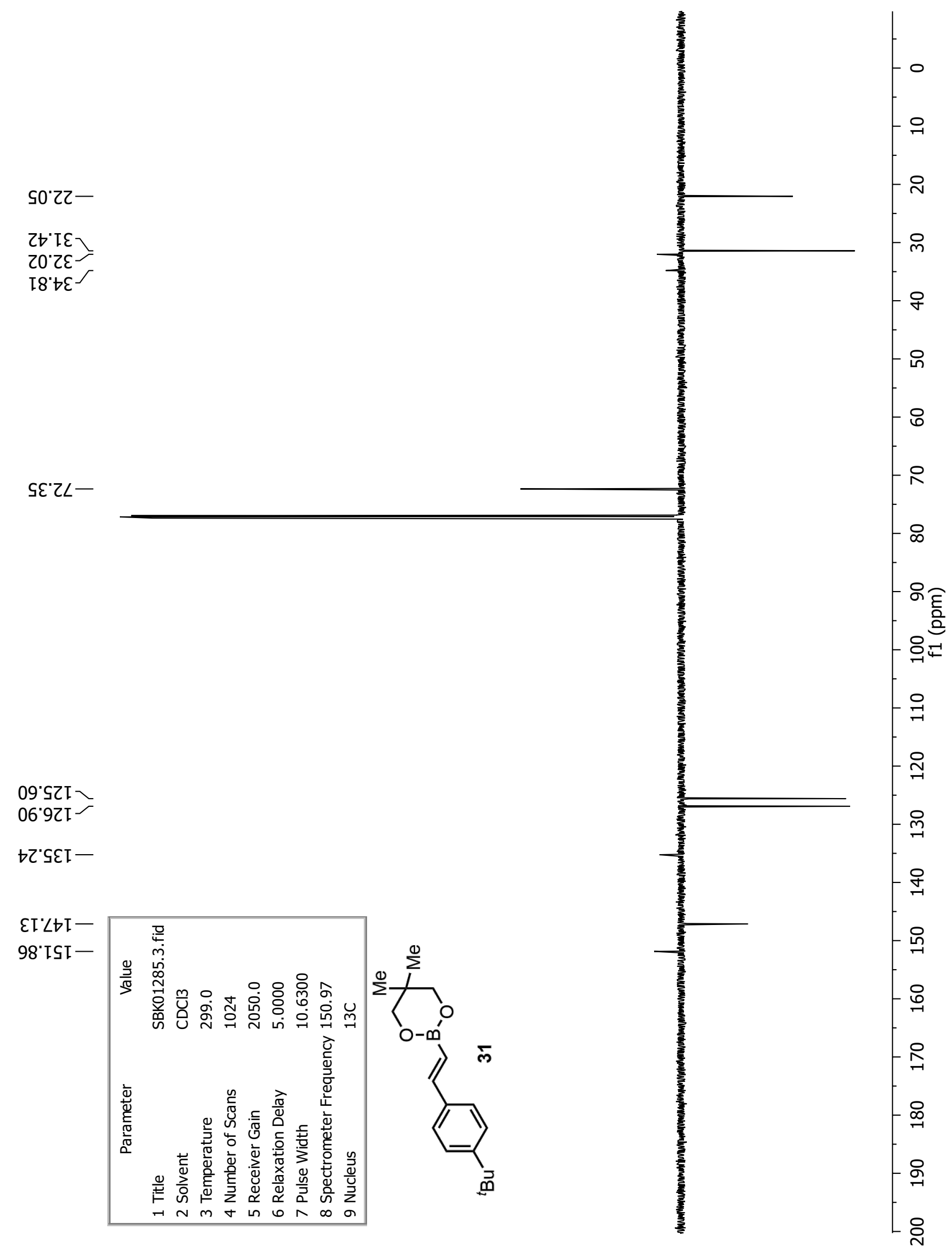




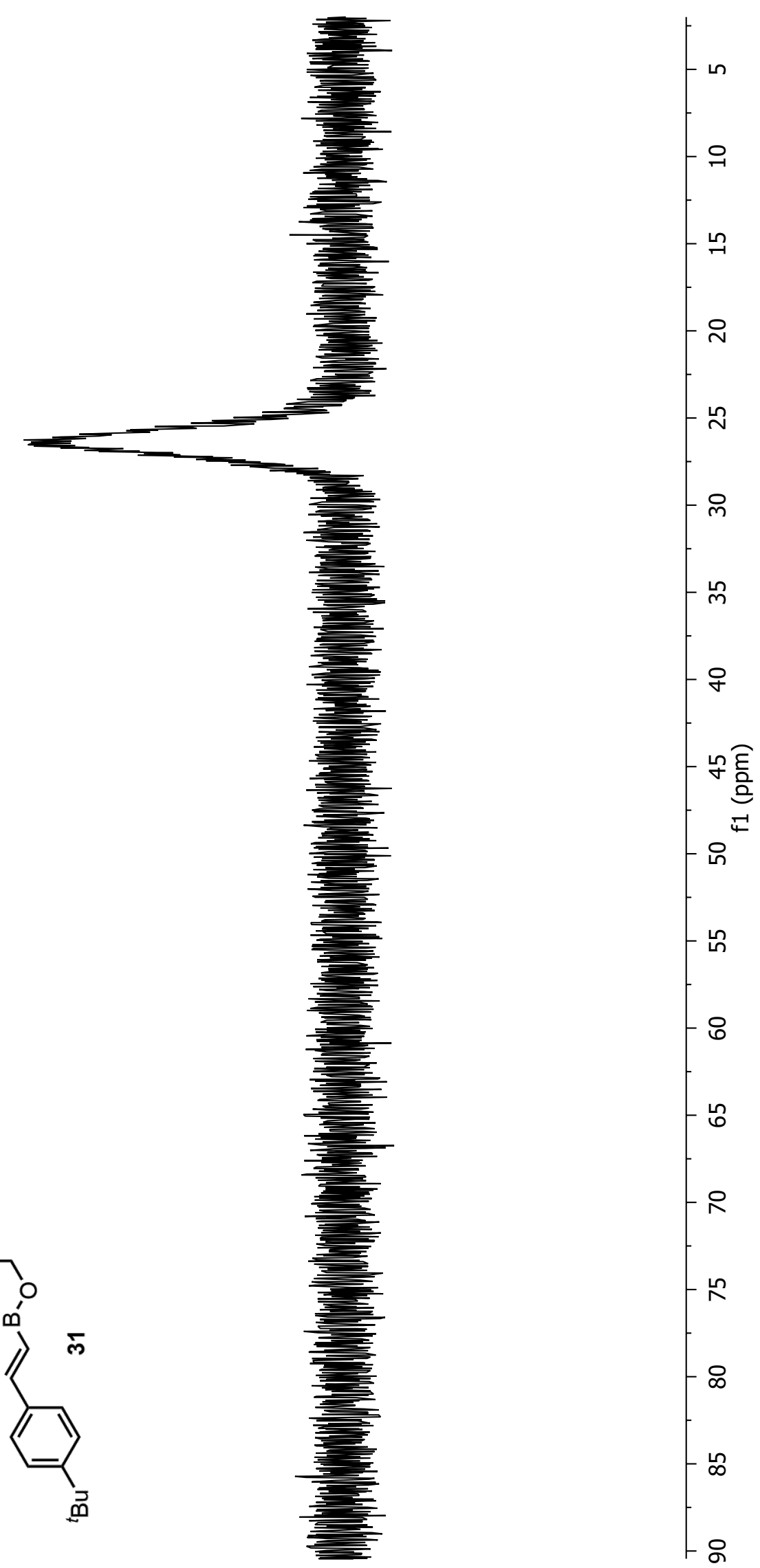




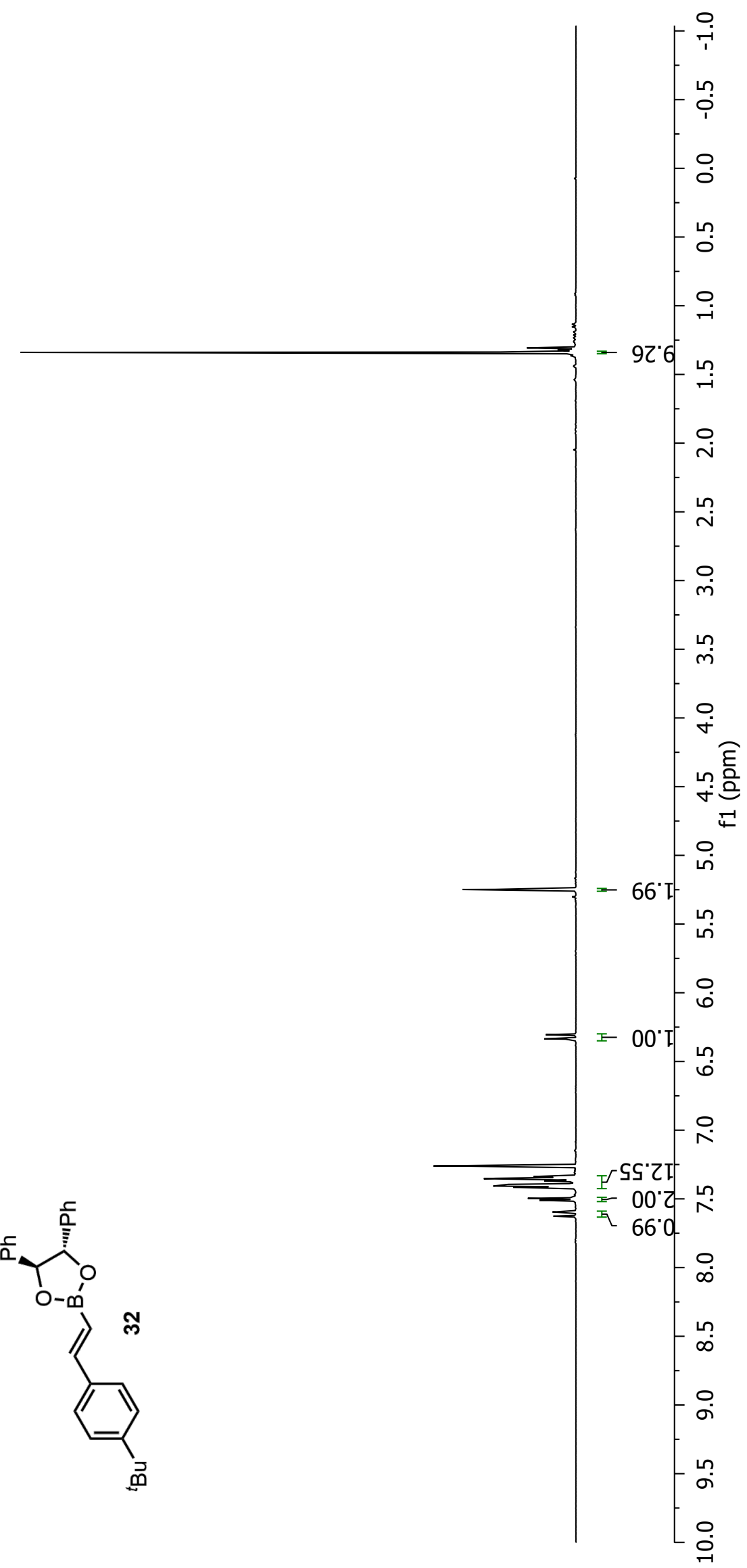

$0 \varepsilon^{\circ} 9$
$+\varepsilon^{\prime} 9$

$9 Z^{\circ} L$

$\forall \varepsilon^{\prime} L$

ऽะ' $\angle$

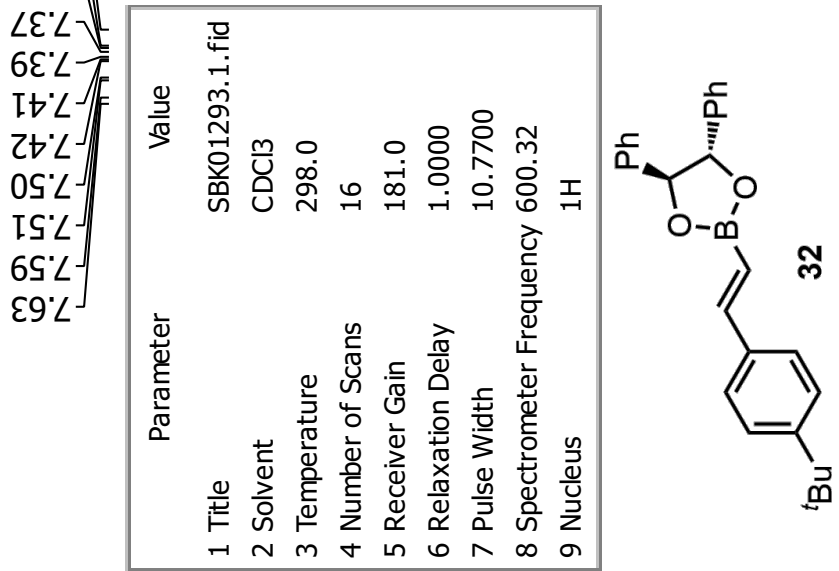




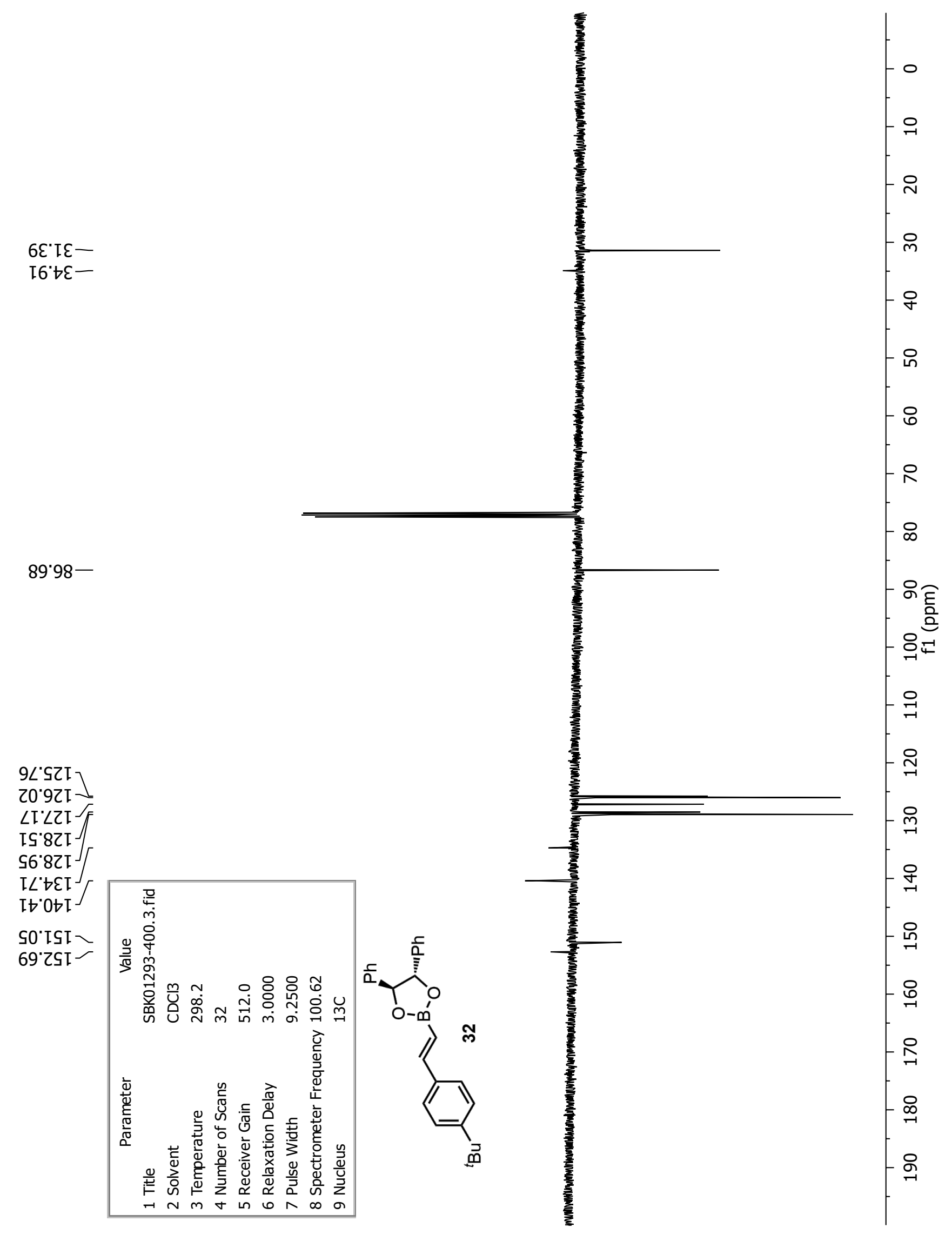



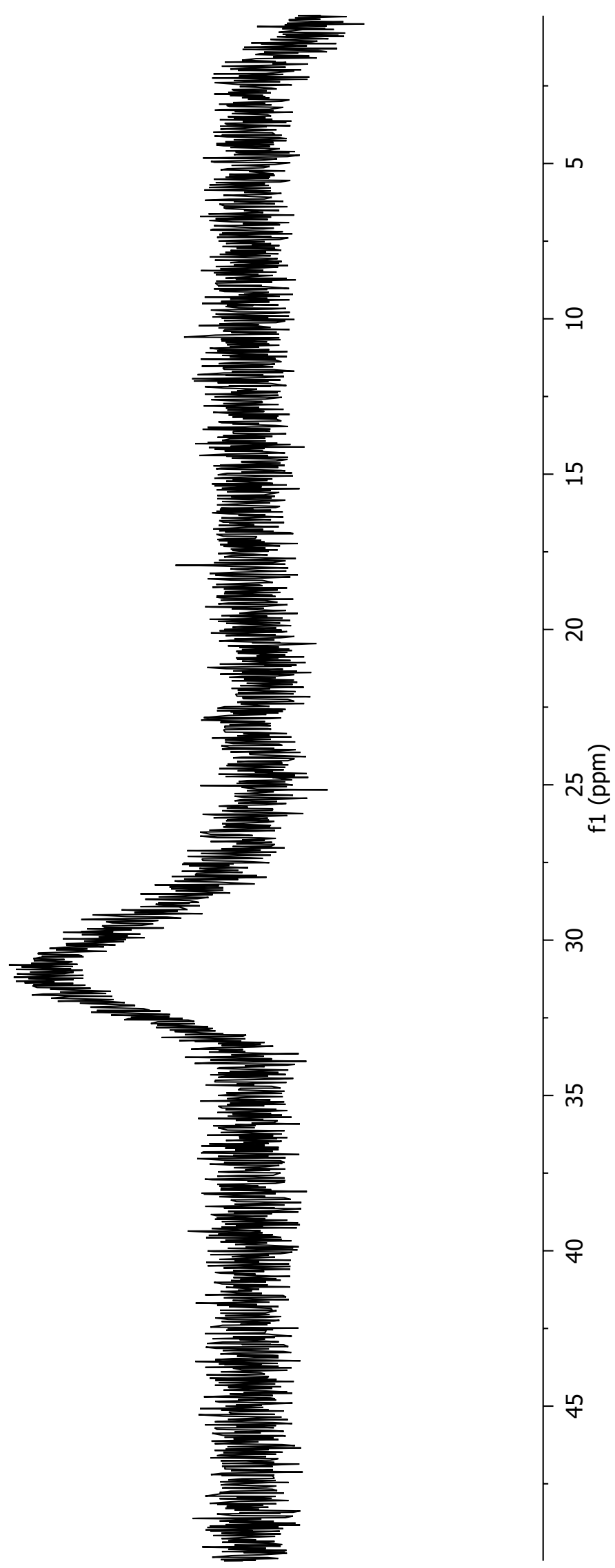


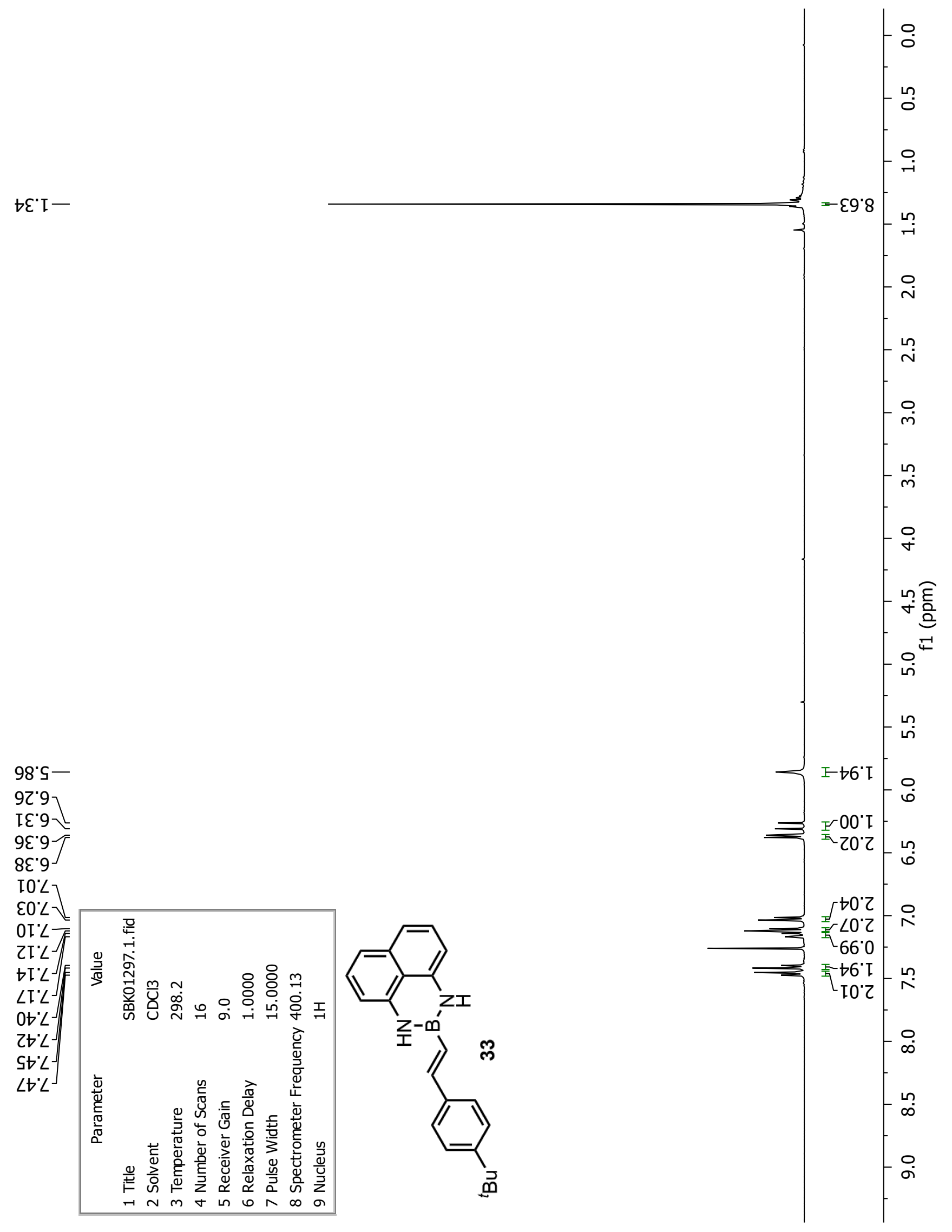


It $T E-$

$98^{\circ} \sqcup \varepsilon-$

S8. SOI-

$\angle 9^{\circ} \angle I I \backslash$

6L'SZI

$\angle 9)^{\circ} 921$ I

EL'LZI

$\varepsilon 6^{\circ} \mathrm{bI}-$

8t.9عI

โE' โ†I

s9. $\varepsilon \pitchfork โ$

OI'ZSI -

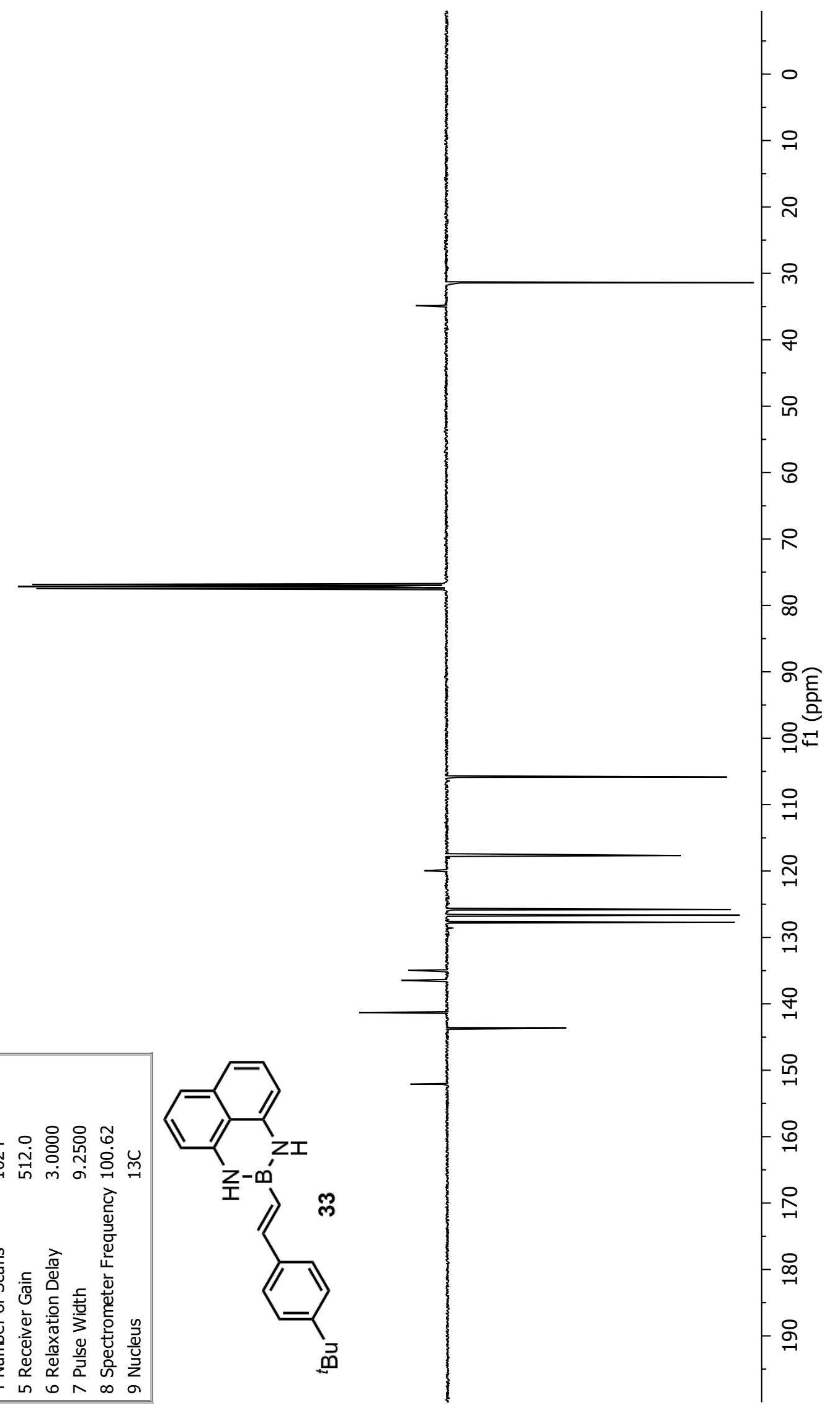



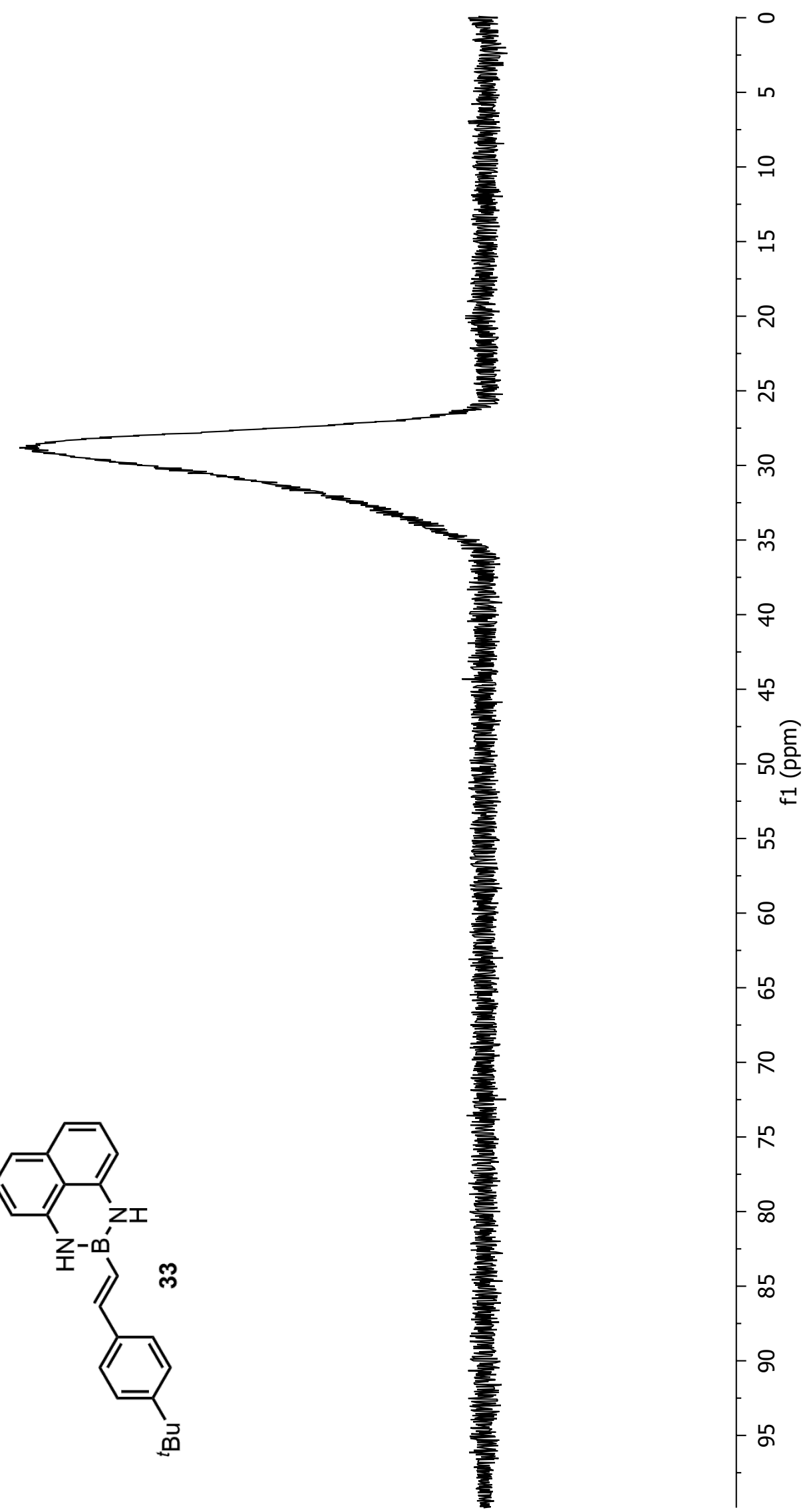

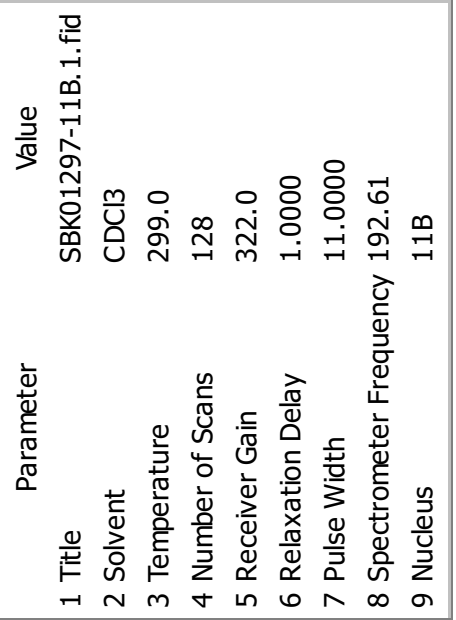

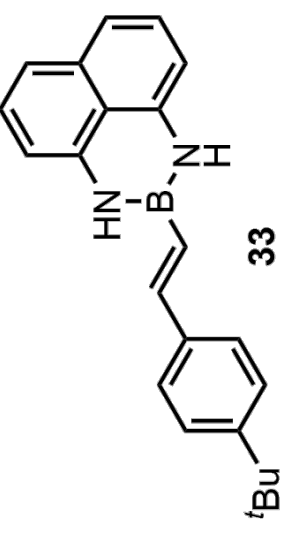




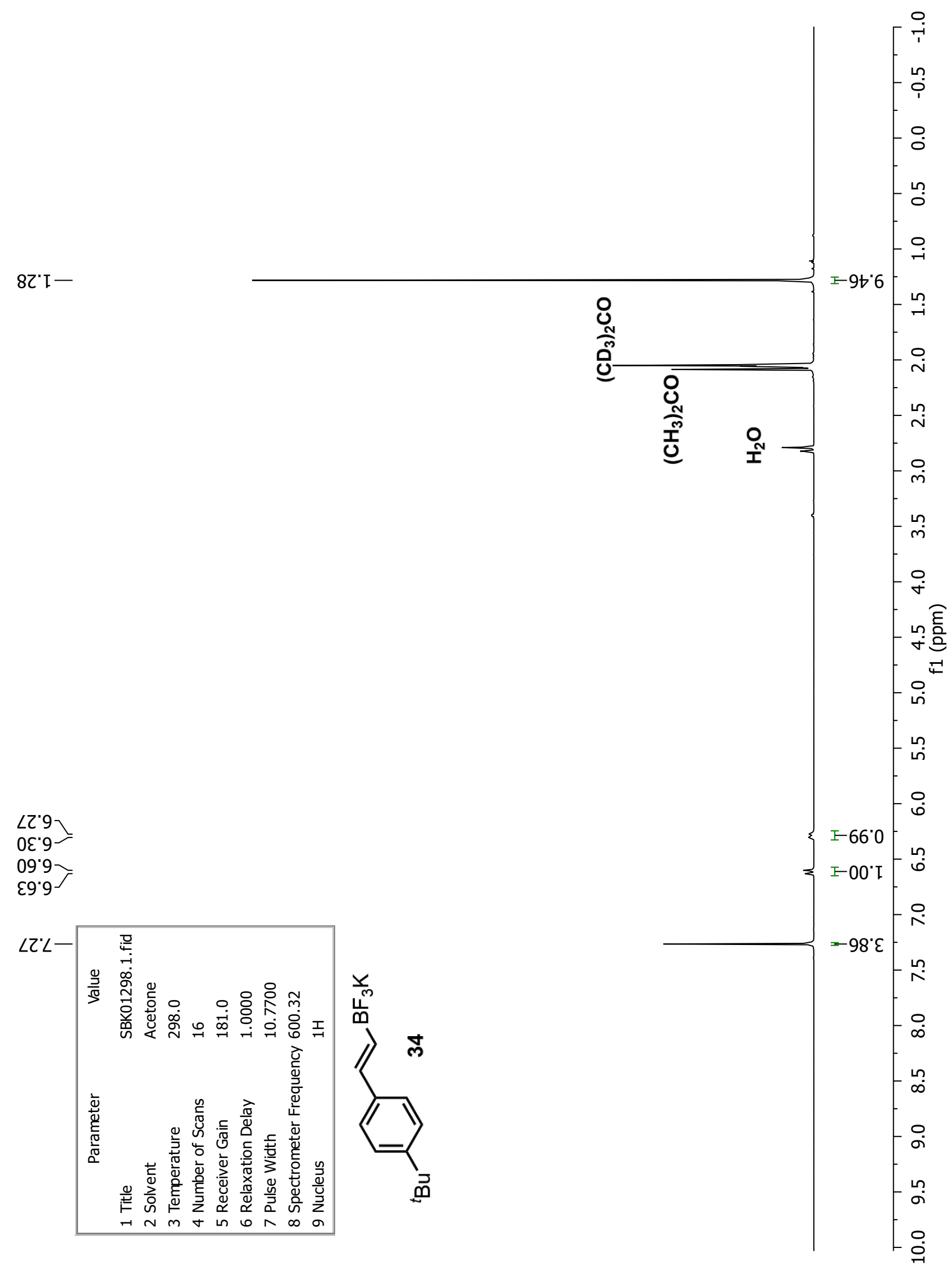




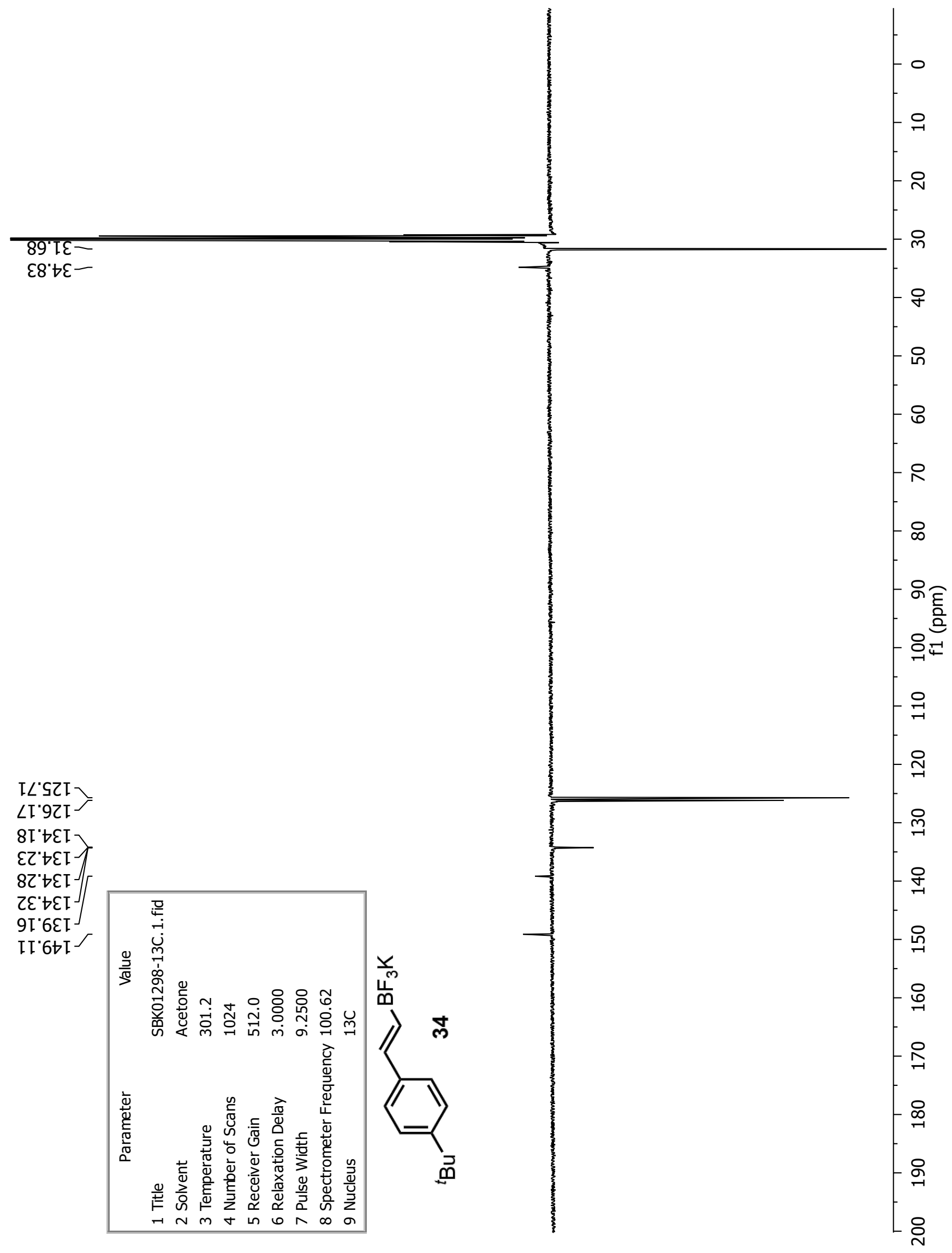




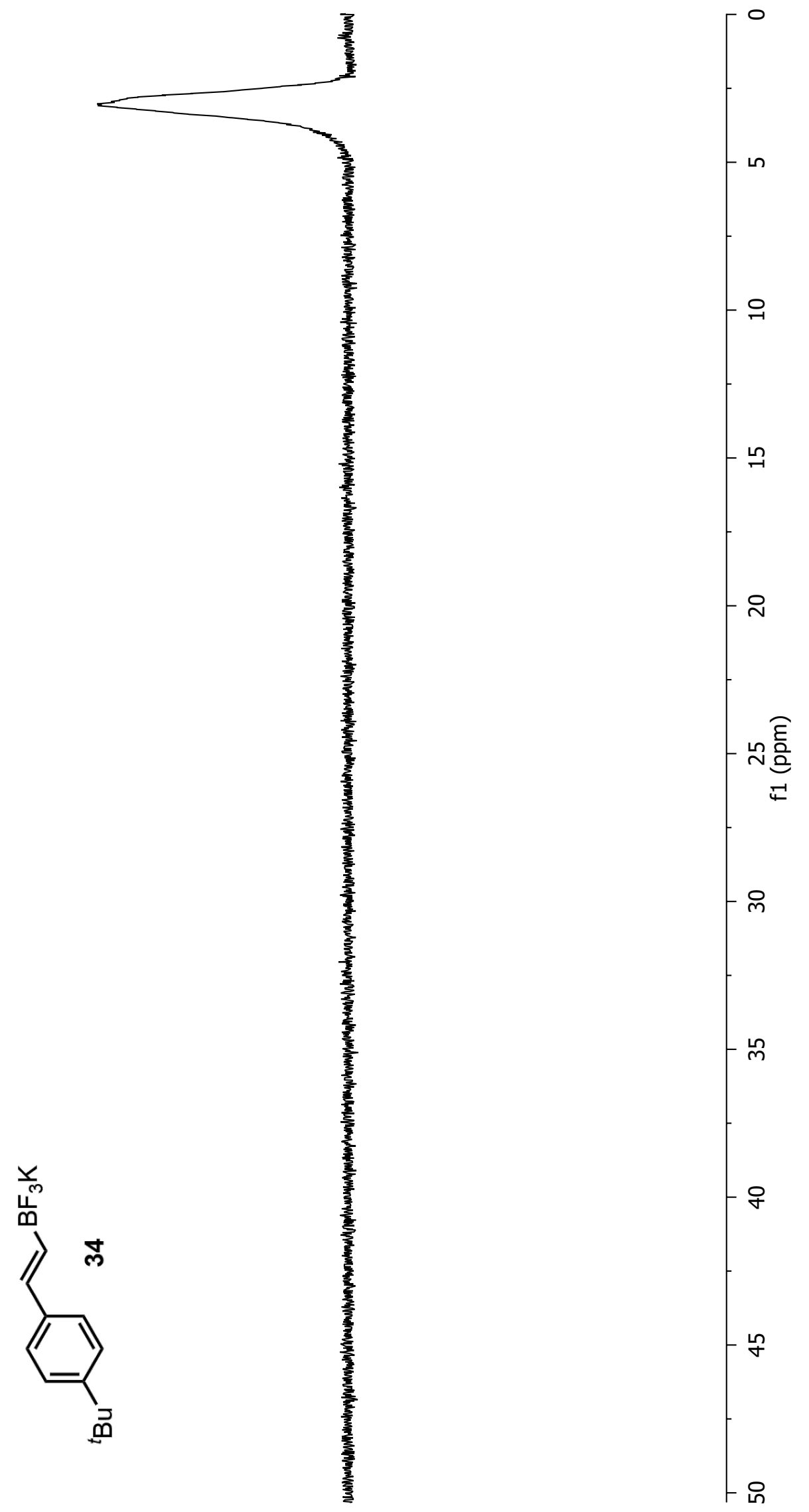




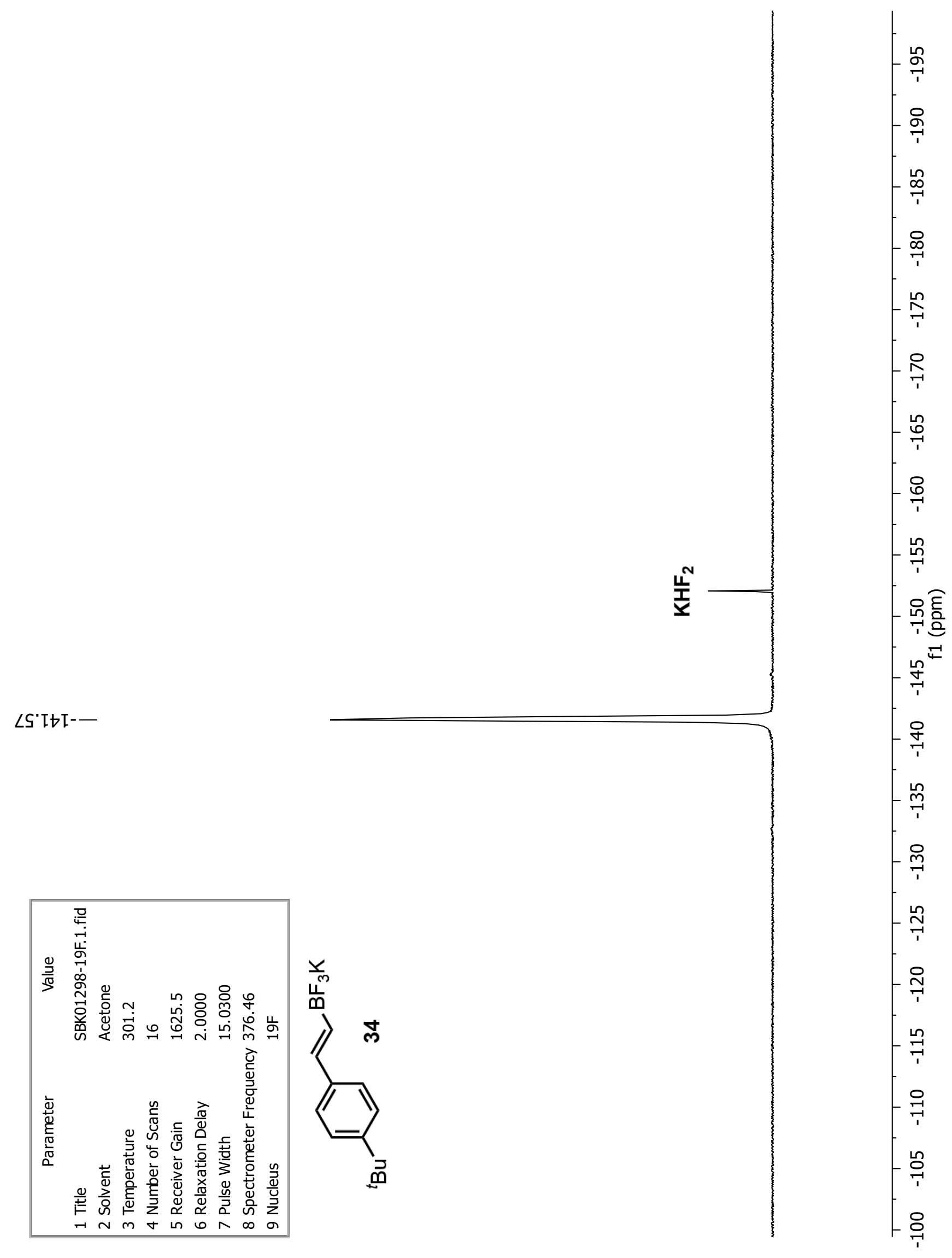

